\title{
Culture and the \\ Economy in the \\ Internet Age
}

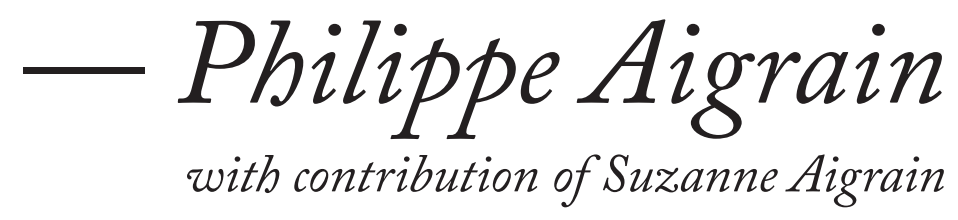

AMSTERDAM UNIVERSITY PRESS 
Sharing 



\section{Sharing}

\section{Culture and the Economy in the Internet Age}

\section{Philippe Aigrain}

with the contribution of Suzanne Aigrain 
The publication of this book is made possible by a grant from the Open Society Foundations.

This book is published in print and online through the online OAPEN library (www.oapen.org). OAPEN (Open Access Publishing in European Networks) is a collaborative initiative to develop and implement a sustainable Open Access publication model for academic books in the Humanities and Social Sciences. The OAPEN Library aims to improve the visibility and usability of high quality academic research by aggregating peer reviewed Open Access publications from across Europe.

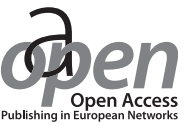

Cover design: Maedium, Utrecht

Lay-out: JAPES, Amsterdam

ISBN $\quad 9789089643858$

e-ISBN 97890485 I 5349

NUR $\quad 983$

Creative Commons CC BY NC ND

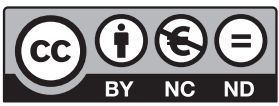

http://creativecommons.org/licenses/by-nc-nd/3.0

(c) P. Aigrain \& S. Aigrain / Amsterdam University Press, Amsterdam, 2012

Some rights reserved. Without limiting the rights under copyright reserved above, any part of this book may be reproduced, stored in or introduced into a retrieval system, or transmitted, in any form or by any means (electronic, mechanical, photocopying, recording or otherwise). 
To Mireille, Tom, Louise and Jonathan 



\section{Acknowledgments}

Building upon a book that was published by Philippe Aigrain earlier in French (Internet \& Création: comment reconnaître les échanges sur internet en finançant la création, InLibroVeritas, October 2008), Sharing: Culture and the Economy in the Internet Age takes into account comments, criticism and suggestions for improvement received from many readers.

The ideas developed in Sharing could not mature in isolation: they are rooted in collective environments. Invaluable inspiration and support were provided by Jérémie Zimmermann and all the participants in La Quadrature du Net, by Juan-Carlos de Martin and the members of the COMMUNIA Network on the Digital Public Domain, by researchers in the Berkman Center for Internet \& Society and the NEXA Center for Internet \& Society, members of the Forum d'Action Modernités, and participants in the Free Culture Forum and the Free Culture Research Conferences.

Some of the findings presented in this book would have been impossible without the large-scale data collection and publication efforts conducted by researchers such as Frédéric Aidouni, Mathieu Latapy and Clémence Magnien of the Complex Networks team of University Paris 6, and Bodó Balázs and Zoltán Lakatos of University of Budapest.

Many individuals deserve a special mention: Sharing would not be what it is without Fernando Anitelli, Phil Axel, Raphaël Badin, Maja Bogotaj, Yochai Benkler, Juan Branco, Paul Branco, Jean-Gabriel Carasso, Aline Carvalho, Mario Ciurcina, Roberto di Cosmo, Milad Doueihi, Mélanie Dulong-de-Rosnay, Christophe Espern, William Fisher, Vera Franz, Mayo Fuster-Morell, Volker Grassmuck, Jim Griffin, Peter Jenner, Gaëlle Krikorian, Hervé Le Crosnier, Olivier Lejade, Lawrence Lessig, Simona Levi, James Love, Eben Moglen, Francis Muguet', Charles Nesson, Jérémie Nestel, François Pellegrini, Valérie Peugeot, Rufus Pollock, Manon Ress, Marco Ricolfi, Gérald Sédrati-Dinet, Tom Smith, Richard Stallman, Malte Spitz, Peter Sunde, Félix Tréguer and Laurence Vandewalle. We remain of course solely responsible for the analysis and proposals developed in this book.

Mireille, Louise, Tom, Jonathan, and many friends created an environment without which this book would not exist. 
Sharing found its home in the publishing world at Amsterdam University Press, an open access friendly publisher staffed with author-friendly editors. We are grateful to Saskia de Vries, Jeroen Sondervan, Chantal Nicolaes, Paul Penman and Alison Fisher for transforming our text into a published book and open access monograph.

This book was drafted and laid out using the Lyx, Latex, and JabRef free software. Graphical illustrations were produced with the Inkscape and Gimp free software or from our own software in Python. Our software and datasets can be downloaded from the book site at http://www.sharing-thebook.net. On the same site, the reader can also run our models with adjusted parameters and upload datasets in order to run our algorithms for the study of diversity of attention. 


\section{Contents}

List of figures

List of Tables $\quad$ I3

I Introduction $\quad 15$

Setting the scene

2 The Internet and creativity debate 21

3 The value of non-market sharing 27

3.I Sharing is legitimate $\quad 27$

$\begin{array}{ll}3.2 & \text { Sharing is useful }\end{array}$

3.3 The media industry opposition to file sharing 43

4 Sustainable resources for creative activities 49

\section{The Creative Contribution}

5 Which rights for whom? A choice of models 59

5.I Access without rights to share 59

$\begin{array}{ll}5.2 & \text { Compensation schemes } \\ & 65\end{array}$

$5 \cdot 3$ Social rights for all 70

6 Defining rights and obligations $\quad 79$

6.I Which works to include 80

6.2 Rights and obligations of users and intermediaries 84

7 How much? 89

7.I Rewarding the present and financing the future 90

$\begin{array}{lll}7.2 & \text { Rewards } & 92\end{array}$

7.3 Financing production and the creative environment 100

$\begin{array}{ll}7.4 & \text { Passing copyright-law tests } \\ 7.5 & 109\end{array}$

7.5 Is the Creative Contribution socially acceptable? 122

8 Sustainable financing for the commons $\quad 127$

8.I Evolution of the Creative Contribution in one country 127

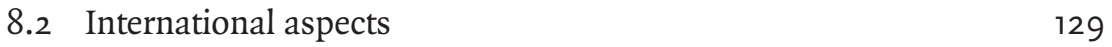

8.3 Economy and non-market commons 130

Implementation

9 Organization and complementary policy measures 137

9.I Principle and essential components 137

9.2 Decision-making processes and democratic governance 139 
9.3 Additional policy measures 141

Io Usage measurement for equitable rewards 145

Io.I A general usage measurement system 145

I0.2 Registration and identification of digital works 149

I0.3 Data collection 152

I0.4 Performance in one medium 153

I0.5 Management costs 155

II Clarification and counter-arguments $\quad 157$

II.I Clarification 157

II.2 Criticisms by opponents 160

II.3 Criticisms by defenders 164

I2 From proposal to reality 169

I2.I Grassroots Internet and creative communities $\quad 169$

I2.2 Government policy 172

I2.3 Policy-makers 173

I2.4 Entertainment players? 174

I2.5 Collective management? 175

I2.6 The continued role of academic research 177

\section{Appendixes}

A Diversity of attention for beginners 181

A.I From wealth to popularity 181

A.2 Testing and parameter estimation for Zipf's law 186

A.3 Zipf's law and diversity of attention in $\mathrm{P}_{2} \mathrm{P}$ sharing 189

A.4 A fresh look at the Long Tail theory 191

B The total cost of rewards and their distribution 193

B.I The model used in chapter $7 \quad 193$

B.2 Reward functions 197

C Modeling usage measurement 199

C.I General model 199

C.2 Music singles in the US 201

C.3 Blogs in France 203

C.4 Fraud prevention and detection 204

$\begin{array}{ll}\text { Notes } & 207\end{array}$

Bibliography $\quad 221$

Index 231 


\section{List of Figures}

3.I Cumulative access (listening or downloads) to the 5565 music tracks available on the Musique Libre site in 2006, normalized for how long they had been on-line [Aigrain, 2006]. p. 38

3.2 Comparison of the cumulated access on the Musique Libre site in 2006, with the cumulated access that would result from the best-fitting Zipf's law (see appendix A for technical details). p. 38

3.3 Cumulated attention for extreme observed cases. p. 39

3.4 Comparison between the observed cumulated attention for the 2 million most popular files in eDonkey sharing and the cumulated attention for a distribution similar to the one studied by Page and Garland [2009] for single commercial downloads, adjusted for universe size. p. 4I

3.5 Cumulated observed access for film sharing in Hungary (I 542 films shared over 3 months in 2008), data from Balázs and Lakatos [forthcoming]. p. 42

4.I Cultural and creative activities and their economy. p. $5 \mathrm{I}$

4.2 Roles and functions in media. p. 52

7.I An image from COMBO, a collaborative animation by Blu and David Ellis, http://vimeo.com/655516r, license CC-By-NC-ND. p. 90

7.2 Various distributions of attention to creators can lead to great differences in the number of creators who share $90 \%$ of the usage credits. p. 95

7.3 Estimate of the 300 highest rewards from the Creative Contribution in the US, for all media in various hypotheses of distributions. p. II2

9.I Core components for the organization of the Creative Contribution. p. 138

IO.I General structure of a possible usage measurement system. p. I46

A.I Share of total wealth held by the $20 \%$ richest individuals, depending on the value of Pareto's law parameter. p. 182

A.2 The number of occurrences of words in a French text of 30,000 words, as reported by Estoup, reproduced in Petruszewycz [1973]. p. I84

A.3 Number of times each track was listened to for the 1000 most popular titles on the Musique Libre Creative Commons music streaming platform in 2006 [Aigrain, 2006]. p. 187

A.4 Share of access to the $80 \%$ least popular works under Zipf's law with the same parameter $(\alpha=\mathrm{r} .0)$ in roo universes containing between rooo and I00,000 works. p. I88

B.I Rewards for the first 20,000 creators among one million rewarded creators for various reward functions. p. 197 



\section{List of Tables}

7.I Number of rewarded contributors and total amount of rewards for various hypotheses on observed diversity or applied reward functions. p. 99

7.2 Estimates of yearly production investment for various media in three countries. p. 105

7.3 The total financial needs for the Creative Contribution. p. 108

7.4 Yearly household cultural expenditure (2007). p. I23

A.I Best-fit Zipf's law parameter, corresponding KS distance for different subsets of the Aidouni et al. [2008] data set. The last two columns give the range of ranks for which the observed access is higher than the best-fitting Zipf's law prediction. p. Igo 



\section{Introduction}

This book is about file sharing ${ }^{\mathrm{I}}$ for creative, expressive or informative works in all media. More specifically, it is about file sharing between individuals and without profit motive. File sharing is the act of making a file available to other individuals by putting it on-line, by sending a copy, or by rendering it accessible through a file sharing software. We defend the view that sharing without direct or indirect monetary transaction - or "non-market" sharing - is legitimate. We also claim that sharing is socially and culturally valuable and will play a key role in the future of our culture and the creative economies. Furthermore, this book proposes a means to strengthen and exploit the synergy between file sharing and creativity, for the general benefit of society and the enrichment of the cultural economy.

Underlying the entire book is an exercise in modeling and empirically studying the popularity of different works in different conditions. How much is this attention concentrated on a limited set of works, or spread over many? We use this analysis to demonstrate the positive impact of non-market sharing for cultural diversity, to reflect on different reward and financing models, to estimate their initial global scope and speculate about their evolution, and to understand how precise the measurement of usage must be for rewards to be fair and respectful of diversity. The analysis and the related models are meant to provide a toolkit for cultural and media studies, usable regardless of whether one agrees with our proposals. Three appendices provide an introduction to these models, explaining their mathematical basis in simple terms, presenting our assumptions, and the empirical studies that support some of our claims.

The book is structured in three parts. The first part sets the general scene.

Chapter 2 provides an introduction to the heated debates that surround the issue of file sharing, and presents the central ideas of the book:

- The non-market sharing of digital works is valuable and must be recognized as a legitimate activity (chapter 3 ).

- New financing schemes are needed to turn the potential of a many-to-all creative world into a reality (chapter 4). In such an environment, all will have access to works, the right to share them and the technical means to produce new works. Many will build new capabilities in informing others, expressing oneself, and creativity. They will catch the interest of some, and some - more numerous that today - will attract the interest of many. 
The second part presents and discusses the principles of the Creative Contribution, a proposal to enable the recognition of file sharing by setting up a new system of rewards for the creators of works that are the object of non-market sharing. The proposal also incorporates support for the production of future works and an environment that nurtures creativity. The idea of linking financial rewards based on contributions by Internet users with the recognition of sharing is not new: it has been the basis of many proposals since 2003. Our proposal is distinguished from them in that it defines both the rewards and the recognition of sharing as social rights, among a society-wide community of contributors who manage the digital cultural commons together. Where others focused on solving a problem facing the cultural industries (the so-called "piracy"), we ask ourselves how could one best enable cultural and expressive activities in a world where non-market sharing is recognized as legitimate.

Chapter 5 compares various models for the development of our digital culture: some which do not recognize the right to share; others which do recognize sharing, but under a copyright-based licensing or tort compensation approach. It then introduces the social right model underlying our proposal.

Chapter 6 delineates the rights and obligations associated with sharing under our proposal: which works are included and when? What are the associated obligations? It discusses the position of those who provide the means to share and of archival organizations in this new context.

Chapter 7 addresses a central issue: how much? What is the total amount of money needed to reward existing works? How much should be dedicated to financing the production of new works and added-value editorial activities? How can creative activities be allowed to grow at a rate which is not constrained by the growth rate of the monetary transaction-based economy? Our choice of approach makes answering this question particularly challenging: we must evaluate both needs and possibilities without the relative comfort of measuring torts or losses of revenues to be compensated. We present models which enable us to set the level of financial rewards and of support to the production of new works directly. We discuss the acceptability of the corresponding amounts to be financed by households from a social and economic viewpoint. In one section, we address the compatibility of our proposal with copyright law requirements, in particular with regards to economic rights. This section exploits recent empirical fact finding on the impact of file sharing on various components of the cultural economy.

Chapter 8 discusses the possible future evolution of the Creative Contribution, and describes its more general relevance as a model for financing the conditions of existence of common goods to which all can contribute, and from which all benefit.

Part III addresses two issues that are central to the implementation of our proposal: how it can be organized (chapter 9) and the critical aspect of usage measurement, which sets the basis for rewards (chapter Io). In both cases, the solu- 
tions we outline are based on empowering individuals in the governance of the system and the production of the necessary data. We evaluate to what degree this approach can meet the necessary precision for the rewards to be equitable, and for the prevention of fraud. We discuss the respective roles of government, nongovernmental organizations, providers and citizens.

Chapter II lists and discusses key questions and criticisms which have been raised in response to previous versions of our proposal. It is presented as a list of Frequently Asked Questions for brevity's sake.

In the concluding chapter, chapter I2, we consider by which paths a proposal such as ours can become a reality. 



\section{Setting the scene}





\section{The Internet and creativity debate}

(I) Everyone has the right freely to participate in the cultural life of the community, to enjoy the arts and to share in scientific advancement and its benefits.

(2) Everyone has the right to the protection of the moral and material interests resulting from any scientific, literary or artistic production of which he is the author.

\section{Universal Declaration of Human Rights, article 27}

Great historical changes, whatever their importance, take place over a relatively long period. Seventy years after the first developments of computer science, and thirty years after the birth of the first world-wide information exchange networks, we are still very far from having a real grasp of their consequences. It takes decades for these technologies to disseminate, and their implications only reveal themselves as humans appropriate them. Many analysts apply outdated models to new activities, analyzing Internet use with tools that were appropriate to study, for example, the impact of photocopying on book publishing. They thus reduce the use of computers to the action of copying. A similarly misleading viewpoint consists in treating Internet merely as a new distribution channel. Both approaches ignore the new ways of interacting with information, which the Internet opens for everyone. These lead to new practices: listening, viewing, annotating, recommending to others, re-using, tailored programming (as in programming a radio or a TV), remixing, and creation. The Internet, coupled with widespread access to computers, provides an environment in which new cultural practices are developed and appropriated by the public. Some commentators, on the other hand, tend to exaggerate the depth of the transformations we are seeing. For example, they believe that some essential processes, such as identifying high-quality items among the abundance of works available, or appropriately allocating resources to creative activities, are now trivial.

This book sets itself the challenging task of being amenable to everyone who has an interest in the Internet, culture and creative activities; of identifying a common framework in which, without necessarily agreeing on everything, we may explore the realms of the possible. However, the reader will have to do some of the work. If you are sincerely convinced that anyone who makes the digital repre- 
sentation of a work of art available on a peer-to-peer network is a pirate (i.e. someone who, by violent means, takes the property of another), and that works of art "bleed" when they are shared, ${ }^{\mathrm{I}}$ this book will not require you to shed those beliefs, but it will expose you to the writings of some, who have another view of these matters. Consider their proposals carefully, and note the care with which they attempt to protect what you hold dear, namely: the recognition and reward to those who partake in creative work; the implementation of channels which enable certain types of works to exist; the access of all to culture. On the other hand, if you believe that, in the age of Internet, collective management is no longer needed, and that if only universal exchanges were allowed to be truly open, they would naturally ensure a better distribution of financial means for creators, reading this book will not require that you change your mind either. But do read what follows, and ask yourselves whether the proposals which are made therein are not the guarantee of what matters to you: the development of Internet-enabled activity, its core freedoms, and the cooperation of all towards a common goal.

Digital technology and the Internet gave us precious resources: new media, each bringing new types of creative work; a new world of cultural practices which do away with the clear-cut distinction between creators and their audiences, producers and consumers; and old and new intermediaries to enable all to appropriate these new practices, and to promote quality in activities conducted in common. We have a duty to nurture this potential, to treat it not as a problem, but as an opportunity.

This book is motivated by a key question:

If we recognize that individuals have a right to share digital works between themselves, how can we make sure that many will be fairly financed and rewarded for producing these works?

This question builds upon a long list of earlier works, and at the same time, it departs from their premises. The idea of associating mechanisms to reward or fund creation with the right to accomplish certain actions using technology is not new. In 1985 , the potential of the Internet was still very hazy and the Web didn't exist. However, legislation was passed in many countries to allow the copying of works for private use, under certain conditions, for instance the payment of a levy on blank carriers such as writable CDs. The corresponding laws framed the right to private copying in a restrictive and narrow manner: they represent an attempt to reach a compromise between limited rights to use digital works and the generation of new resources for creative work. As early as 1992, Richard Stallman, the founder of the free software movement, proposed to use a tax on digital tape recorders and their magnetic cassette tapes that was being discussed at the time for a better allocation of funds to artists whose works were copied (Stallman 1992). 
After the birth of peer-to-peer $\left(\mathrm{P}_{2} \mathrm{P}\right)$ file sharing with Napster, and facing a fierce reaction from the music industry, a number of proposals were made for collective licensing mechanisms in the US in 2003 and 2004 that would authorize those paying a monthly fee to freely exchange digital music (Netanel 2003, Von Lohmann 2003, Von Lohmann 2004). William Fisher's book Promises to Keep: Technology, Law, and the Future of Entertainment (Fisher 2004) explored in depth many possible approaches for the remuneration of creation in the digital world, and many of the later proposals revolved around his ideas. In 2005, a blanket license proposal (applying to music and film) was tabled in France under the name licence globale by the 'Alliance Public Artistes', ${ }^{2}$ and supported under various guises by members of the French parliament ${ }^{3}$ from all across the political spectrum. In both cases, these proposals were drawn up in the context of severe and pressing threats to the freedom of on-line exchanges, in the form of highly repressive proposed legislation, the application of which was foreseen through mechanical technology or automated justice. The blanket licensing proposals were designed in a hurry, and their main failings, as we will see, were a mixture of timidity in endorsing sharing and a lack of adaptability to the specifics of certain types of media. Since then, several writers or public interest organizations have proposed various flavors of collective licensing mechanisms for peer-to-peer or other ways of sharing digital works in both Europe and the US.

The thoughts and proposals developed in this book owe a lot to these precursors: ${ }^{4}$ they build on their approaches whilst attempting to overcome their limitations. Most of the earlier work was preoccupied with how one could compensate the music and film industry for the pains of having to operate in a world where the public can act as a distributor of works in its own right. We ask ourselves a different question: how can one make sure that cultural production and the search for quality in all media are sustainable in a world where many more will engage in it? Authors, performers, and contributors of all kinds will only support the recognition of the right to share if credible approaches to the sustainability of cultural activities are on the table.

Actually, gaining this support may not be the most difficult challenge: it might be at least as difficult to convince every citizen that there is a need to put in place new financing schemes for creative activities. Over the past 15 years, the public has been treated as an enemy by a never-ending stream of laws and policies intended to eradicate the unauthorized sharing of files representing copyrighted works between individuals. This sharing was described as an act of piracy, and ever more extreme means were put in place to prevent or punish it. The aim of this book is not to recount all the legal measures, technical devices, new means to incriminate, or compulsory propaganda in favor of some business models adopted in this period, nor to describe the generalized confusion between justice, police, administration and private stake-holders which they have installed. ${ }^{5}$ None- 
theless, even parties that cannot be suspected of anti-copyright extremism have expressed strong warnings against the dangers of the war against sharing.

This happened, for instance, after a handful of multinationals of music and film led a world-wide campaign to introduce three-strike approaches, under which people alleged to have shared digital works without authorization are banned from the Internet after two warnings. The best-known examples of implementation of three-strike approaches are the two laws on "Création and Internet", also known as HADOPI laws, adopted in 2009 in France. ${ }^{6}$ After the first was adopted by the French parliament, it was challenged in the Conseil Constitutionnel (CC 2009) as being contrary to fundamental rights. This court, officially recognizing that access to the Internet is a necessary condition for the freedom of expression and communication which is essential to democracy, declared that this access can only be restricted following a decision by a court of law, while the bill entrusted this sanction power to an administrative authority. The second law circumvented this by instigating a semi-automated justice system based on penal ordnances. In 20II, the UN Rapporteur for freedom of opinion and expression (LaRue 20II) clearly condemned the principle of sanctions that deprive people of access to the Internet. He also expressed concern about the latest trend in antipiracy: making intermediaries that provide access to information liable for the use of their services to share copyrighted works.

Another example is the Anti-Counterfeiting Trade Agreement (ACTA). This text, negotiated between 2008 and 2010 among "like-minded countries", was presented as a trade agreement, but it contains provisions for criminal sanctions for copyright piracy. It is widely regarded as a circumvention of democracy for the benefit of a limited interest group. Steward Baker, who was Assistant Secretary for Policy in the US Department of Homeland Security in 2008, described (Baker 20II) his concerns at the time as follows: "It seemed as a sweetheart deal for a few intellectual property owners, who'd get free government enforcement of their private rights, potentially to the detriment of security and traditional customs enforcement. Worse, the sweetheart deal would be written into international treaty, putting it beyond Congress's reach if the risks we foresaw actually came to pass." Finally, data protection authorities such as the European Data Protection Supervisor (EDPS 2009, EDPS 2010) have issued repeated warnings about the risks of anti-piracy measures for privacy.

Without lingering on what could be a very long list, one must take stock of the hurdles that this "war on piracy" has created for the acceptance by citizens of new proposals for a society-wide financing of creative activity. There is a widespread perception among citizens that laws and policies are adopted for a benefit of a few; that, as individuals, they are stigmatized for conducting acts that they do not see as harmful; and that the rights of authors are invoked to protect a few corporations or interests such as heirs of deceased artists whose contribution to the future of culture is disputable. The present schemes of collective management of 
royalties or fees are seen as opaque and unfair to most contributors to creative works. As a result new proposals for financing schemes are scrutinized with a justified caution.

Some think that there is no need to do anything, and are reluctant to impose new financial burdens on individuals. They argue that sharing will go on, as it is a natural thing to do once the possibility arises, and impossible to prevent when close to two billion people have the technical means to copy and exchange works. Artists themselves will increasingly permit those who like their works to share them with others. As we have done with this book, they will for instance put their works under Creative Commons licenses that authorize users to copy and redistribute digital works. As we will see, this laisser faire attitude may be too optimistic. For some media, a few companies and organizations have such a hold on the promotion and distribution of works that they are able to dissuade creators from authorizing sharing. More generally, making sharing a crime will not stop it, but it might prevent it from achieving its cultural potential. Certainly, staging a war between creators and the public - two increasingly intermingled categories - will not help in building a new social contract under which all contribute to the culture of tomorrow.

There is potentially a high price to pay if we let interest groups and organizations without democratic control define the environment of creativity on the Internet: by delaying the construction of the many-to-all cultural society, we risk being dispossessed of our common future. As citizens or as stakeholders, we would do better to debate our future ourselves, and on our own terms. Policy-makers will then be in a position to have an informed discussion on how to amend the law and policies, in order to recognize the best of today's practices and possibly turn some of our proposals into reality. 



\section{The value of non-market sharing}

\subsection{Sharing is legitimate}

Sharing used to be beyond the copyright arm

We are all accustomed to a dogmatic view of copyright, which is more about forbidding certain things than ensuring certain outcomes. For those who promote this view, the idea of allowing people who are neither the authors nor the copyright holders of a piece of work to share it with other individuals is tantamount to heresy. Article 27.2 of the Universal Declaration of Human Rights (quoted earlier) should serve as a reminder that this has not always been the dominant view. To interpret this article in its fullest sense, we must take into account any means of promoting the material and moral interest of the authors of works, not just the control of copies. With this open approach in mind, is it so obviously wrong to transmit or to make available a cultural product in a non-profit way? Just how could this harm culture itself, or those who contribute to it?

When works could only be distributed on a physical substrate, the first sale doctrine (also known as the exhaustion of rights doctrine) acknowledged that, after the sale of a cultural good, the person or organization acquiring it was free to transmit it to another person. This doctrine was actually just codifying a longstanding principle: copyright (or author rights for that matter) was not concerned with what individuals who have entered into possession of a work such as a book do with it. It was easier to adopt this view in past times because the carrier and the information it carried could not be easily separated. Nonetheless, the usage this enabled was far from insignificant: it led to the development of many useful activities - lending or giving books and records to friends, but also creating loan libraries for books and other media, or videocassette and DVD rental centers. Activities such as reproducing extracts in notebooks, or cutting and pasting them in the physical sense, were widely practiced in the Renaissance and classical periods (Blair 2010). Twenty years ago, when a few lobbies started their great campaign to enforce the scarcity of works in the information domain, they were all too aware of the dangers that these past practices posed to their theories. They thus proceeded to attack them, targeting for example loan libraries in Europe. The economic effects of lending books are limited or inexistent, but these lobbies wished to erase all precedent of a right to share works without their permission. 


\section{Information technology and non-market exchanges}

Today, information and communication technology (ICT) can be used for activities which, previously, required the creation, manipulation or transport of a physical object, or that were simply impossible. One can now create a piece of work, share it with others, annotate it, comment on it, by exchanging only information. This change concerns not only the artistic or cultural domain, it also affects scientific and technical practices, management, machine design, inter-personal communication, public expression and the media.

Most disagreements on intellectual rights can arguably arise from different takes on the changes introduced by ICT. What the latter allow, first and foremost, are exchanges and collaboration on a very large scale, with minimal transaction costs. 'Transaction costs' include costs linked to monetary transactions, contracts or any other type of agreement, but also the cost of detecting the necessary skills for a project, or of reaching the public interested by a given content. These extraordinary benefits are only realized, however, if information exchanges are 'free' (i.e. not subjected to prior agreements, transactions, authorizations or pre-use controls). It is thus in the 'non-market' sphere that the advantages of the information revolution are most evident: access to works and knowledge and evaluation of their interest, distributed co-operation towards the production of informational tools such as software, collaborative media, etc.

Nowadays, the true disagreement no longer concerns the radical nature of the changes introduced by ICT, but rather the acceptance of their effects. Some consider it desirable to impose, in the information domain, the scarcity and degree of control which were unavoidable in the sphere of physical carriers. They argue that this is needed in order to preserve certain functions which existed in the latter sphere (investment into the production of certain contents, remuneration of the authors, sign-posting of interesting content). It is our intention to demonstrate that, on the contrary, freeing up non-market exchanges between individuals can have a generally positive impact on culture and the creative economy.

Computers and the Internet have made it possible to exchange works on a much larger scale, without depriving the original owner of access to them. Thus, an activity widely recognized as useful - sharing a work of art or opinion with someone else - becomes possible on a much greater scale. Does that suddenly make it harmful? In terms of providing a channel for access to culture and knowledge, it can only be an improvement. However, some parties adamantly reject sharing, 
equating it with criminal activities such as stealing and piracy. They must have a reasonable motive for doing this. Just what does sharing harm in the modern sense of sharing information or files? What it really threatens is exclusive control of the supply of works. The conflicts surrounding culture and the Internet intensify around one issue: in tomorrow's world, who will determine which cultural works reach the public and how? In the era of large, centralized cultural industries, it was not authors and other contributors, but large publishers and distributors, who had an almost exclusive control on distribution. To understand how this situation is likely to evolve and what challenges this evolution might imply, we must first explore in more depth what sharing is.

The potential afforded by the sharing of information has been largely realized in certain areas, such as the open Web in general, public expression in blogs and collaborative media. We owe it a major regeneration of democratic processes. In other domains, such as photography, music and video communities, we have live experiments in voluntary sharing of works by their authors, using Creative Commons or similar licenses. Finally, we have a giant file sharing laboratory, using dozens of tools and technologies, for commercially distributed copyrighted works, but also for the on-line archiving of public radio and TV programs, or the provision by members of the public of rare or orphan works. ${ }^{2}$ So large a flow of cultural exchanges between individuals is unprecedented, and hence it is worth a closer look.

\section{File sharing}

\section{Accessing and sharing contents}

The publishing industry thinks in terms of access to contents: will people download digital works? Will they access them through streaming (a technology that enables users to listen to or view contents stored in a central server without downloading a copy)? For the industry, sharing is just another way of accessing contents without their permission. However, sharing between individuals leads to very different practices in comparison to downloads or streaming from centralized sites: when individuals decide what to make available to others (and this could be all the documents they have in digital form), what they share directly reflects their preferences. By contrast, on centralized sites, there is a bias towards specific contents which are made more visible than others, either through advertising or because many other people are accessing them.

There are many ways to share contents beyond peer-to-peer file sharing. Swapping USB keys, for instance, is a popular way of sharing digital works today. At first sight, it suffers from the same limitations as sharing physical books did in the past: the USB key has to travel physically from one individ- 
ual's computer to another. However, this exchange is quite efficient in practice, because USB keys now have large capacities, enabling them to hold large sets of works, and because just about everyone in developed countries (and soon elsewhere) is equipped with them. The old practice of making contents one likes available on a personal website has become less frequent, because of the risk of being charged with copyright infringement. Newsgroups, which are a form of email list servers whose messages are sent to subscribers, existed well before the Web, but remain a very efficient way to obtain some contents "on request" among communities interested in specific contents, which often are no longer accessible easily on the commercial market.

At the end of 1998, a young student called Shawn Fanning started developing Napster, a system to share $\mathrm{MP}_{3}$ music files among individuals. Napster started operating in June 1999. At its peak, the system had more than 25 million users and 80 million files. ${ }^{3}$ Works in various media were shared using the Internet long before Napster, ${ }^{4}$ but Napster was responsible for making file sharing - as an expression and as a practice - popular amongst the general public. The importance of Napster lies both in its architecture and its philosophy. Napster had some flaws, which made it an easier target for law suits. For instance, it was based on a single central register of which users hosted which file. However, it was a true sharing tool, where access to a file was obtained by an individual from other individuals, a principle that came to be known as peer-to-peer file sharing, or $\mathrm{P}_{2} \mathrm{P}$ for short. ${ }^{5}$ Napster launched the idea of personal music library pooling systems, where users have access to the music libraries of all other users. Pooling libraries is an old dream, already present in antiquity. The Ptolemies implemented it in a somewhat centralized and confiscatory manner: every ship landing in Alexandria was required to hand over any papyrus scrolls on board to the Library of Alexandria, where they were kept, the original owners receiving only a copy (Philips 2010). The Renaissance humanists practiced it in a more civilized manner by exchanging copies between themselves. As for modern libraries, some countries have revived a softer version of the ancient rule, by requiring a legal deposit of a copy of all published works in one or more libraries. However, the need to move or store objects physically is a significant hindrance for centralized or pooled libraries. Digital technology and universal information networks have now removed this limitation. At the time of writing, the Comparison of File Sharing Applications page ${ }^{6}$ on Wikipedia lists some 60 applications (not all active at present), but these only represent some of the many ways to share digital works. If the nonmarket sharing of digitally published works is recognized as legitimate, other ways of sharing files between individuals that are presently too risky in terms of 
prosecution will become possible again, such as simply putting digital works online on a personal website. ${ }^{7}$ More generally, file sharing practices will no longer occur in a semi-clandestine fashion, their practitioners will no longer be called bad names or be subject to surveillance and pursuit by private police organizations. The information sphere will no longer be polluted by fakes in the name of the war on peer-to-peer networks.

We must thus analyze not just what sharing is today, but what it could be in a different situation, where more people are able to work towards quality in sharing: quality of the digital representation of works, of their attribution to authors and contributors, of the tools available to search for them, to identify those with interesting content and flag them. Of course, not everyone is interested in contributing to quality improvement, but it is enough that a few are, and are able to work openly: new services and intermediaries emerge, and reputations are made.

\section{Fakes}

Fakes are files purporting to contain a given work, whereas their content is in fact different (for example a short excerpt that is looped over and over). Most fakes are deliberately injected on behalf of publishers, who call upon the services of specialized companies to wage this war on peer-to-peer networks (this practice is called $\mathrm{P}_{2} \mathrm{P}$ warfare). If non-market exchanges were recognized, files which mask one content with another would probably continue to exist (for example to disseminate pornographic content), but it would be much easier to detect and avoid them. Far from fighting this latter type of abuse, those who oppose file sharing currently exploit it in order to discredit what they object to. Unfortunately for them, MediaDefender, a market leader for the injection of fakes on behalf of the major companies, was caught red-handed running a parallel business in fakes which redirected users to its own paying pornographic sites (Salliou 2008).

\subsection{Sharing is useful}

The information age is one where many more people engage in producing contents in various media and expressing themselves towards an open public than ever. As we will see in section 7.2 , II to $20 \%$ of the population older than 15 in developed countries engage in producing contents for sharing on the Internet, and this proportion is constantly on the increase. A reasonable cultural policy must endeavor at making a many-to-all cultural society sustainable, at ensuring that each human being can contribute to and participate in such a society, accord- 
ing to their wishes and abilities. ${ }^{8}$ Sharing is useful because it contributes to this perspective in many ways.

\section{Sharing as cultural empowerment}

The first useful quality of sharing is obvious, though it is often forgotten even by its advocates: sharing is not the same thing as access. If its adversaries do not speak of sharing, but rather of piracy or illegal/unauthorized downloading, streaming and access, it is because they are well aware that a direct attack on sharing would be easy to criticize. Sharing is an act of making something available to others, just like - in a more minor way - recommending a work to someone or - in a more involved way - re-using one in a creative process. This is why, even when one is not the author of a digital work, sharing it with others is a step towards cultural empowerment. This step is particularly important, because it can be practiced by all, at a very limited entry cost.

How much sharing with how many people?

Curiously, little attention was paid to the fact that uploading or making works available to others through a $\mathrm{P}_{2} \mathrm{P}$ network requires resources. A full music album, compressed using the FLAC lossless compression codec popular among demanding file sharers, represents 340 megabytes. A decent quality $\mathrm{MP}_{3}$ version represents ${ }_{4} 0$ megabytes. Assuming a bandwidth of 5 I2 kilobits per second (a realistic estimate of the true upload bandwidth available on average to broadband Internet subscribers in developed countries), it takes ig to 45 minutes to upload such an album once. Even if a broadband connection is used only for this, only rooo to 2250 albums can be uploaded per month. In practice, $\mathrm{P}_{2} \mathrm{P}$ networks allow the user to limit the upload bandwidth they consume. A typical choice for a "good sharing citizen" is 40 kilobits per second, corresponding to a maximum of 80 to 200 music albums uploaded per month (and far fewer movies). Of course, future technological advances will raise these limits, but the idea of one person directly making millions of files available remains a complete fantasy. Note that this reasoning also applies to protocols such as BitTorrent, which allow users to obtain different parts of a file from different sources: the compound upload time remains the same. We will see that some protocols are favorable to cultural diversity, while others are less so. Using the diversity-prone protocols, many people can - together - share a very large common library, but each shares only some works with some people. This also explains why USB key swapping, despite its physical location limits, remains an attractive way of sharing files. 
File sharing is fundamentally different from streaming, because through the former, one comes into possession of a copy of the works. This copy can be searched, read, listened to, or viewed ad lib, with whatever tools one chooses. This is not just a matter of convenience, it also enables specific activities: comparison, analysis, criticism, or re-use. Most significantly perhaps, sharing empowers Internet users by enabling them to act as a distributor, as a relay for the dissemination of a work. This is so important to them that many are prepared to devote significant money and time resources to sharing.

\section{Cultural diversity}

Cultural diversity has many dimensions: how diverse are the works that are produced? How many creators contribute to them? By how many channels are they distributed? How many languages are represented and to what extent? Some of these dimensions are difficult to assess, not least because the diversity of works cannot be reduced to an objective measure. Others can be misleading: the increase in the number of television channels has not necessarily increased the diversity of sources of contents, because the contents that receive the most attention actually come from a limited number of sources, for instance companies such as Endemol that design "formats" of shows that are then "customized" for given countries. In this book, we focus only on two dimensions of cultural diversity: the range of works that are accessible to users in practice, and the diversity of attention given by users to works in various media.

Sharing contributes to cultural diversity first by enlarging the set of works that are made accessible to the public at a given time in a given geographic area. To take stock of the immense changes that have already happened in this arena, it is useful to distinguish between 4 types of on-line cultural, informational or expressive contents:

- material that is de facto shared voluntarily by authors without explicit licenses, where non-commercial sharing by individuals carries no practical risk of copyright litigation;

- material that is explicitly submitted to licenses that authorize at least noncommercial sharing;

- material that is orphan, is no longer or never was distributed commercially, or was produced by public organizations, and which is shared by individuals without authorization;

- commercially distributed material shared by individuals without authorization.

Legally inclined readers might find the distinction between the last three categories surprising: all three cover copyrighted material, and sharing it without authorization constitutes a copyright infringement, unless some fair use, fair 
dealing, exception or limitation applies. ${ }^{9}$ However, our purpose here is to chart different forms of sharing of digital works as they developed on the Internet. If certain practices have been accepted or tolerated by authors, or treated leniently by judges when possible, this might indicate that they are perceived as useful. ${ }^{10}$

De facto sharing without explicit licenses was the first large-scale form of sharing on the Internet. The success of the Web as an information and knowledge sharing platform was based on the fact that people put on-line huge amounts of valuable material, in forms that allowed for it to be easily linked to, copied, pasted, sent to others by email, and often reproduced on the Web itself. As described in (Benkler 2006), this gave birth to a giant non-market sphere of information and knowledge activities. The world-wideWebSize site ${ }^{\mathrm{II}}$ computes on a daily basis the number of Web pages indexed by search engines, which generally means that their contents can be easily copied. At the time of writing, the figure for Google is of the order of 30,000 million. Of course, not all of these web pages can be considered to be shared de facto, but a significant proportion certainly is. It is interesting to note than their number is probably of the same order of magnitude as the number of Internet users... or the number of human beings.

During the first years of development of the Web, the media industry largely ignored it. Retrospectively, it seems that it simply did not fit their world view, precisely because of its non-market character. Hollywood, for instance was obsessed with digital technology at the time, but in form of DVDs and their copy protection systems. ${ }^{12}$ The industry lobbied to obtain a legal protection against circumvention of anti-copying technology - making it illegal to work around copy-prevention technology in order to do the copying. This was first met by a rebuttal from FCC in 1994, but the Clinton administration then pushed it through the World Intellectual Property Organization (WIPO), where it was included in the 1996 WIPO copyright treaties. ${ }^{13}$ Meanwhile, the first license explicitly authorizing sharing at least for non-commercial use, the Open Content License, was released in July $1998,{ }^{14}$ soon followed by the GNU Free Documentation License ${ }^{\mathrm{I5}}$ in March 2000, the Licence Art Libre (Free Art License) in July 2000, and the Creative Commons Licenses ${ }^{16}$ in December 2002. As we have already mentioned, the unauthorized sharing of copyrighted material underwent something of an explosion in the same period with the birth of Napster.

For media where it was widely adopted, voluntary sharing greatly increased the number of works made accessible to the public, under terms that authorize copy and redistribution, and often free re-use with properly signaled modifications, for non-commercial or even commercial purposes. On the Flickr site ${ }^{17}$ alone, more than 175 million photographs are shared under Creative Commons licenses, II5 million of which can be reused with modifications. Even if a judgment on quality is always difficult, particularly on such a large scale, a significant fraction of these photographs seem to be of real quality and interest, even though the site's policy leads to many images being available only at resolutions up to ro24 by 768 pixels. 
The interested reader might experiment by searching for photographs on any given subject on the Creative Commons part of the site. ${ }^{\mathrm{I}}{ }^{\mathrm{B}}$ Blog posts, scientific publications, and on-line encyclopedias such as Wikipedia are other examples of domains in which voluntary sharing has considerably extended the range of accessible contents. Overall, there are several hundred million on-line documents under free sharing licenses: a 2010 estimate reported 350 million works for Creative Commons licenses alone (Cronin 2010).

The situation is different for some media that preexisted the Web, in particular recorded music and moving image, and more recently books. There, certain players have such a degree of control over the commercial distribution, promotion and revenue sources that they can dissuade many artists or producers from practicing voluntary sharing. In some cases, dissuasion is replaced by prohibition: collecting societies for music in Europe almost always require their members to give an exclusive management mandate for all rights on all works. This effectively forbids authors from explicitly authorizing non-commercial sharing of their works between individuals. In these domains, unauthorized sharing, in particular $\mathrm{P}_{2} \mathrm{P}$, plays a key role in extending the range of works that are accessible to the public. In the data collected by (Aidouni et al. 2009) regarding sharing traffic on an eDonkey server during ro weeks in 2008 , no fewer than 275 million files were made available by users. Most of them are likely to be music, as moving image sharing had already moved largely to BitTorrent sharing at that time. Not all of them are shared without authorization: $\mathrm{P}_{2} \mathrm{P}$ networks are used to share government data, free software... or self-published books. It is not easy to know how many different works these files represented. During the ro weeks of the study, users obtained 40 million different file identifiers in answer to their queries, I2 million were actually downloaded more than once. A safe estimate is that no fewer than Io million different music tracks (songs) were made available for sharing. ${ }^{19}$ Thus, this form of sharing alone made more tracks available than the compound commercial offers at the time. ${ }^{20}$

Unauthorized or tolerated sharing is of particular importance for orphan and out-of-publication works, categories that cover a very significant share of our culture. A great proportion of copyrighted works are orphan or out-of-publication works: these represent an estimated two-thirds of books, for instance (Brantley 2009). The proportion is lower for recorded media such as music, moving image documents being in an intermediate situation. ${ }^{2 \mathrm{I}}$ Though this may seem strange, some public organizations have also turned a large part of our public domain cultural heritage into a new form of property: heritage organizations such as libraries, museums and archives, often totally or predominantly funded by the public, claim exclusive rights on the digitized versions of these works, and fail to give access to them under conditions that respect the rights of everyone towards the public domain. Though there are recent public policy efforts to ensure a better accessibility to orphan, out-of-publication and public domain works, 
the various forms of sharing can be credited with important successes in these matters. Volunteers and not-for-profit projects have scanned, OCR-ed, or re-typed and formatted significant collections of public domain works in the Internet Archive OpenLibrary project, ${ }^{22}$ WikiSource ${ }^{23}$ and Project Gutenberg. ${ }^{24}$ Many more orphan or out-of-publication works are accessible on file sharing networks, in proportions that vary significantly depending on the sharing protocol (see below, page 42). The wide diversity of contents available in file sharing has led to practices that are not possible in commercial contexts: comparison between numerous performances for songs or classical music, constitution of specialized personal music collections.

We now need to investigate another facet of sharing: if many works are available, is the actual access to these works truly diverse?

\section{Attention diversity}

The popularity of works has been studied for various media for as long as a century. To do so, researchers measured the popularity of works, for example, the number of times a given book was requested in a library. They then plotted this number, ranked by decreasing popularity, showing the most popular on the left, and the least popular on the right. In large real-world situations, popularity diagrams of this type are not readable, as the curve becomes very close to the axis. To see what is going on, one has to use logarithmic scales, or even better, to plot the cumulative popularity. The cumulative popularity expresses the number of requests for access for works down to a specific popularity rank. We coined the expression "diversity of attention" (Aigrain 2006) to designate a property of access to or usage of works that had long been recognized as important: how much is the attention that people give to works spread over many works or concentrated on a few? If the diversity of attention is large, the cumulative popularity curve increases gradually throughout the range of popularity ranks. On the other hand, if attention is concentrated almost exclusively on the most popular works, the cumulative popularity curve rises sharply at first, then flattens off. In recent years, the focus has shifted towards studying the diversity of attention to digital and online works, though the methodology remains similar.

Traditional publishing of books, records, video tapes or disks selects a limited number of works and tries to maximize their commercial distribution. However, the digitization of all media and the spread of the Internet have strongly decreased the effort and cost of producing and distributing copies of works. This has given birth to the decentralized sharing of works between individuals, and has also led to new commercial publishing models (on-demand publishing, commercial download sites, streaming) both legal and illegal. How do these various models affect the diversity of attention? We started studying this issue in 2005, focusing first on the comparison between voluntary sharing communities on the Internet and the commercial distribution of books, records and DVDs that were 
predominant at the time. Figure 3.I illustrates the key aspect of these studies: they are concerned with how much the works of intermediate popularity, those that do not belong to the I to $5 \%$ most popular titles, but rather to the following $30 \%$, receive attention. In the example of figure 3.I, the music tracks in the [4\%-34\%] most popular titles range receive close to $43 \%$ of the total access, a figure that is, as we will see, very high, indicating a very diverse attention. The idea here is that the titles that are not the most popular but did receive some attention (and thus are likely to be of some interest at least for some users) are the reservoir of cultural diversity. ${ }^{25}$

Of course, our choice of $4 \%$ and $34 \%$ is somewhat arbitrary. To avoid such arbitrary choices, researchers have long tried to characterize the shape of popularity distributions by a single parameter that would provide an objective estimate of the diversity of attention. An historic advance was made in the I930s when George Zipf (Zipf 1935), a Harvard linguist interested in the study of the frequency of words in languages, formulated a law that was found to apply to many real-world popularity distributions. Zipf's law can be formulated as the level of access of $n^{\text {th }}$ most popular work being proportional to $\frac{1}{n^{\alpha}}$ where $\alpha$ is a parameter that can vary. Similar laws have been found to apply in many domains, such as the wealth held by individuals or the size of cities. In appendix A, we provide a comprehensive historical and technical background on these models that are at the heart of debates on the so-called Long Tail theory proposed by Chris Anderson (Anderson 2004, Anderson 2006, Anderson 2009), for instance. Fascinatingly, in many real-world situations the parameter $\alpha$ was found to be close to I, which led to a - false - popular belief that it is always the case, and that in any form of cultural access, the $20 \%$ most popular works always receive more or less $80 \%$ of the attention (which became known as "the $20 / 80$ rule"). In our example of figure 3.I, the $20 \%$ most popular musical tracks receive only $46 \%$ of access.

As one can see in figure 3.2, the best-fitting Zipf law is not always a perfect approximation of a popularity distribution. The exact reasons why popularity distributions and other ranked distributions follow Zipf's law, or deviate from them slightly, are still an object of speculation: see appendix A for our own tentative explanations. Many factors combine to generate the observed spectrum of access to works.

In the many types of commercial distribution or non-market sharing schemes we have studied, we have found that the best-fitting Zipf laws had parameters ranging from 0.5 , for voluntary information sharing communities such as the Musique Libre site mentioned above, to I.4I for the sales of albums published by the music majors in France in 2004 or 2005 (Moreau et al. 2006). These figures correspond to extreme differences in diversity of attention, as illustrated in figure $3 \cdot 3$. 


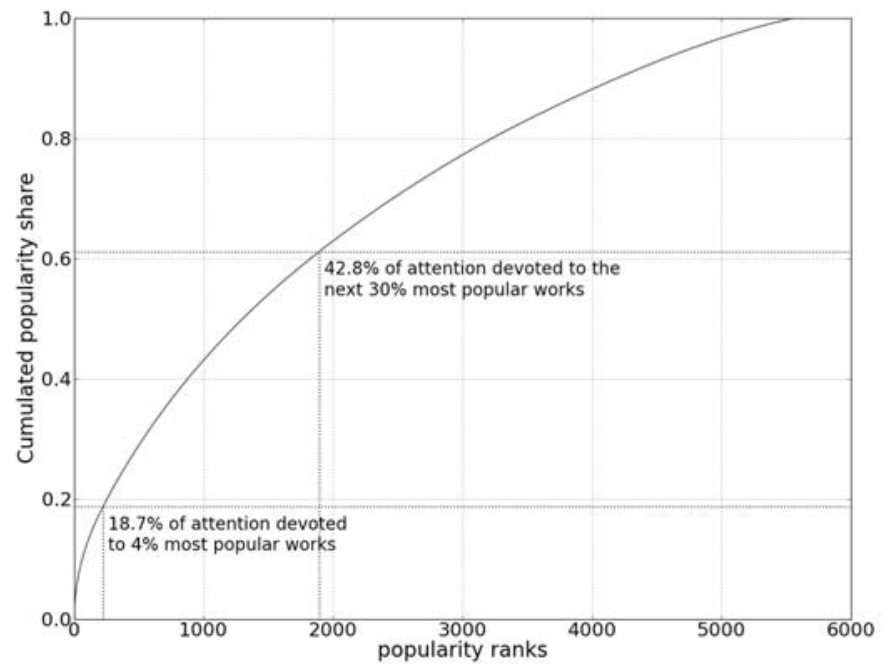

Fig. 3.1. Cumulative access (listening or downloads) to the 5565 music tracks available on the Musique Libre site in 2006, normalized for how long they had been on-line (Aigrain 2006).

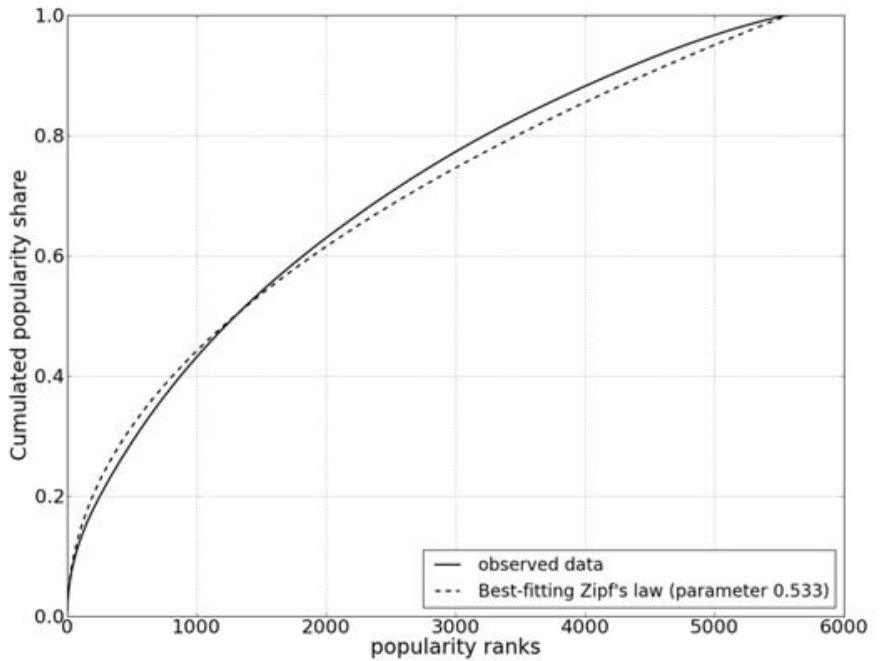

Fig. 3.2. Comparison of the cumulated access on the Musique Libre site in 2006, with the cumulated access that would result from the best-fitting Zipf law (see appendix A for technical details). 
For advertising-funded commercial sites or commercial sales, the full distribution of access to works is not made public, as it is considered to be sensitive commercial information. As a result, only very partial information is available to researchers. One of our key policy recommendations is that the publication of the distribution data for rights collected by collecting societies from each type of source should be required by law. Until such a policy is in place, researchers have to work with partial information, occasionally made available under the form " $\mathrm{x} \%$ of works represents $\mathrm{y} \%$ of sales" or "the $\mathrm{N}$ most popular works received $\mathrm{y} \%$ of access". From this data and information about the size of the universe of works, one can estimate the corresponding Zipf's law parameter and derive the full distribution of attention curve. This is of course an approximation, but in our opinion it is a decent one, and one that will hopefully be validated further in the future.

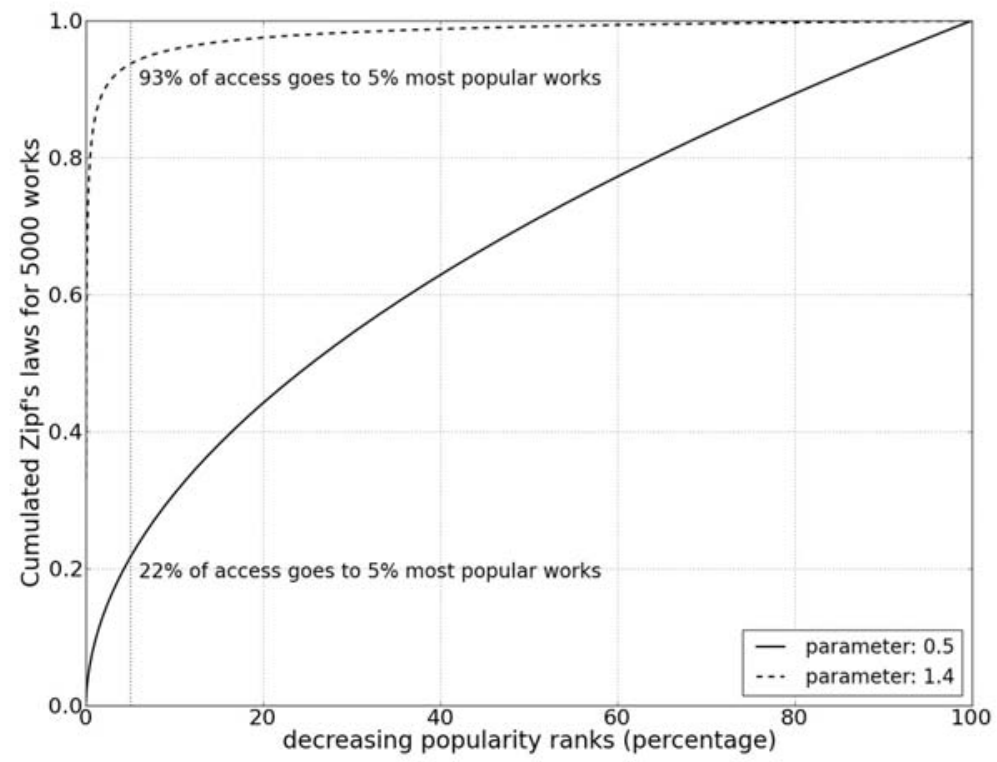

Fig. 3.3. Cumulated attention for extreme observed cases

For the on-line commercial distribution of works, where the number of titles is much larger than for published CDs, the available information on the diversity of attention is even scarcer. Will Page, the Chief Economist of PRS for Music, the music collecting society in the UK, and Eric Garland, CEO of the music industry journal BigChampagne published a curve for an unstated "legal single downloading site" in which $5 \%$ of works generate $90 \%$ of revenues among a set of 1.5 million 
titles (Page-Garland 2009). We derive from it a cautious estimate that the distribution of sales would correspond to a Zipf law of parameter I.I: ${ }^{26}$ more diverse than sales of CDs by the majors, but still very concentrated.

What about full-scale file sharing? Thanks to a remarkable data collection and publication effort conducted by Mathieu Latapy and his colleagues at Université Paris 6 (Aidouni et al. 2009), we were able to study the diversity of access to files in a large segment of $\mathrm{P}_{2} \mathrm{P}$ sharing. They collected data exchanges through one of the eDonkey servers during a ro-week period in 2008. No fewer than 90 million users were involved in sharing using this server in this period, and 12 million files were downloaded at least once. Analyzing this data from a diversity of attention point of view faces three challenges:

- Several files, each characterized by a file identifier, can correspond to the same work. In a later study on the BitTorrent $\mathrm{P}_{2} \mathrm{P}$ file sharing of movies in Hungary, Bodó Balázs (Balazs-Lakatos 2010) "crowd-sourced" the huge task of mapping files to works: he called for volunteers to share the workload and obtained the complete results in only a week. However, this was possible only because his study was focused on film, and considered only a few tens of thousands of files. Two useful lessons can nonetheless be drawn from Bodó Balázs' study: the average number of files per work was around 5 , and the observed distribution of attention for files and for works was relatively similar.

- The eDonkey data have been rendered anonymous, in a way that makes it impossible to select only works in a given medium, such as music.

- eDonkey file sharing is heavily polluted by fake files (Aidouni et al. 2009, Lee et al. 2006), which results in Zipf's law being quite a poor fit to the distribution of access, except when considering only a few hundred thousand most popular files.

More details of our analysis are given in appendix A. We have chosen to focus on the 2 million most popular files in the eDonkey sharing. These files received $94 \%$ of all access, and they are likely to contain a high proportion of music (as film, video and TV sharing had already switched to BitTorrent by the time, see Oberholzer-Strumpf 2010, p. I2). The average number of files per work is probably lower than for film, as there are no duplicates due to language versions, and users quickly select the best quality file for a given work. In figure 3.4, we compare the observed distribution of attention in $\mathrm{P}_{2} \mathrm{P}$ file sharing with the distribution mentioned above for commercial single downloads. The diversity of attention distribution presented here for $\mathrm{P}_{2} \mathrm{P}$ is only an approximation of the one for only individual music works. ${ }^{27}$ However, the huge difference in attention for intermediate popularity works (6o\% of all access versus 10\%) leaves no room for doubt: even if our estimates are revised in later studies, the strongly increased diversity of attention in this type of $\mathrm{P}_{2} \mathrm{P}$ file sharing compared to commercial downloads will still 
hold. These findings are consistent with those reported in a study using interviews of sharers (TNO 2009). However, we will see below that not all forms of sharing have so positive an effect on the diversity of attention to works: BitTorrent sharing appears to lead to a more concentrated access to works.

Once sharing is recognized legally, the diversity of attention in sharing will be subjected to contradictory trends. On one hand, it will become higher, because sharing will no longer be clandestine: when one can expect the sharing commons to remain accessible without risk, it makes sense to make rare works available, and to expect others to do the same, whereas at the moment the stigmatization and repression of sharing focus it on high-demand works. On the other hand, commercial players will come to realize the importance of being visible in file sharing, will aim their promotion at it, and this could lead to a greater concentration of attention in some of the related channels.

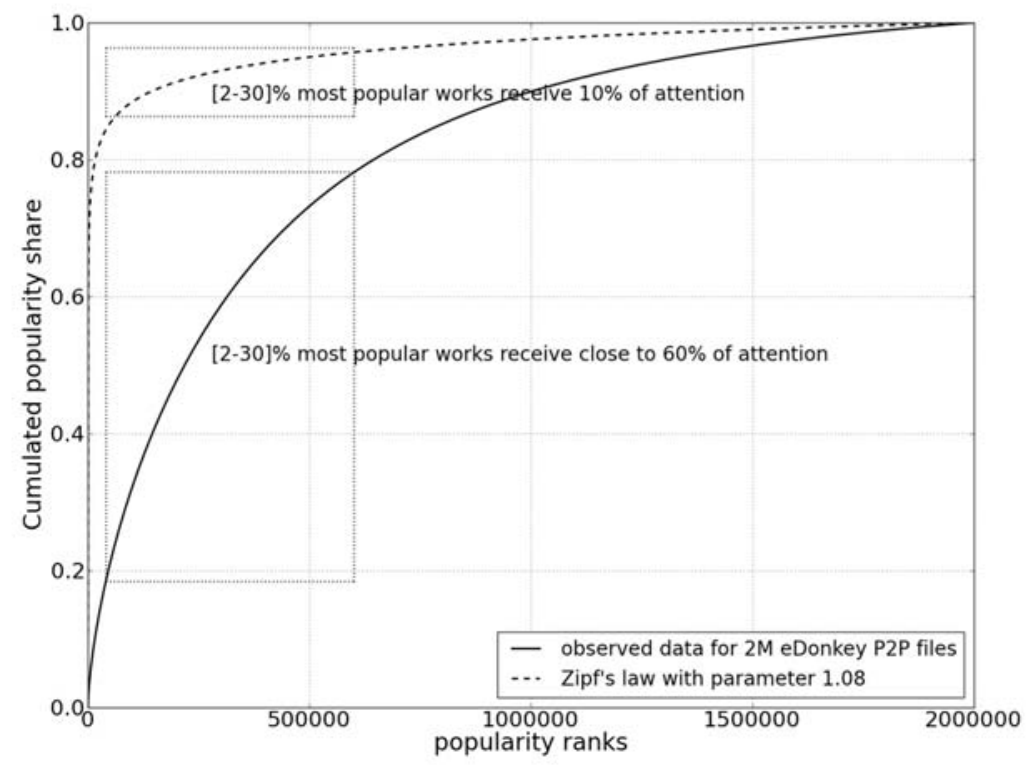

Fig. 3.4. Comparison between the observed cumulated attention for the 2 million most popular files in eDonkey sharing and the cumulated attention for a distribution similar to the one studied by (Page-Garland 2009) for single commercial downloads, adjusted for universe size.

\section{Attention diversity varies with forms of sharing}

There are important differences between the different ways of sharing digital works in terms of their impact on cultural diversity: studies show that eDonkey/ eMule $\mathrm{P}_{2} \mathrm{P}$ file sharing using the eMule protocol leads to a greater diversity of 
attention than sharing using BitTorrent tracker sites. In their study of music, (Page-Garland 2009) also studied sharing through a peer-to-peer protocol which they didn't specify, but that appears to be BitTorrent. From the curve they presented, the distribution of access has a level of diversity similar to a Zipf law with parameter around I for a universe of I.5 million works: the 95\% least popular works get only $24 \%$ of access. (Envisional 20II) recently studied sharing for all media on the PublicBT BitTorrent tracker. This study raises some methodological questions: for instance, they studied one single day of sharing. Their study leads to similar concentration estimates: among the I,48I,479 torrents that were downloaded at least once, those which were downloaded more than roo times, that is $0.439 \%$, account for $30.4 \%$ of all access. This corresponds to a Zipf law parameter of approximately I.04. (Balazs-Lakatos 20II), in their aforementioned study of BitTorrent film sharing in Hungary during 3 months in 2008, collected high quality anonymized data to which they gave us access. ${ }^{28}$ Figure 3.5 plots the cumulated distribution of access for this data. The 95\% least popular films obtain 4I. $2 \%$ of access. At first sight, this might seem much more diverse than the results from (Page-Garland 2009), but we are here in a much smaller universe of 1542 films, where this corresponds to a Zipf law with parameter $0.965 .{ }^{29}$ It seems indeed that BitTorrent sharing leads to a significantly stronger concentration of attention than other forms of $\mathrm{P}_{2} \mathrm{P}$ sharing.

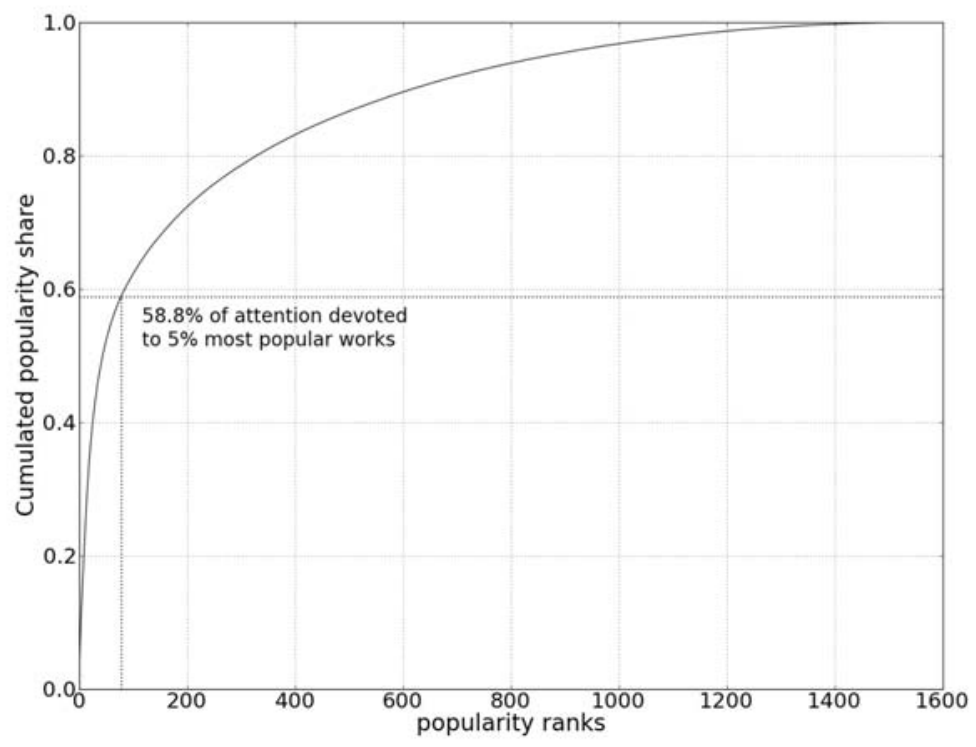

Fig. 3.5. Cumulated observed access for film sharing in Hungary (1542 films shared over 3 months in 2008), data from (Balazs-Lakatos 2011) 
Researchers have proposed explanations of why BitTorrent is less favorable to diversity of attention. Bodó Balázs and Zoltán Lakatos noted that: "Unlike $\mathrm{DC}++^{30}$ file sharing hubs that usually prescribe a minimum amount of data to be offered in a shared library, BitTorrent trackers require that a user balances his/her upload/download ratio around I.o. This technical setup has serious implications on how content is distributed and consumed on each network. Users around $\mathrm{DC}++$ form large, searchable archives, where the amount of data shared is a source of pride and recognition. BitTorrent, on the other hand discourages the emergence of large individual shared libraries as such large libraries offer little reward in terms of the valuable upload ratio." (Oberholzer-Gee-Strumpf 2oro, note I6) commented in another study: "The concentration of movie downloads in part reflects the current BitTorrent technology. Index sites, which list the files available for download, typically de-list a title when no one is sharing ${ }^{3 \mathrm{I}}$ a complete copy for some length of time. As a result, less popular movies become often unavailable, as are older movies since the number of shared copies tends to decline over time."

Though it is hard to obtain reliable information about schemes such as USB keys, downloading from personal sites, or access through newsgroups, we expect them to be strongly favorable to diversity of attention.

\subsection{The media industry opposition to file sharing}

The overall impact of sharing, and more generally of information and communication technology, on the cultural economy will be discussed in chapter 4 , and specifically in sections 5.2 and 7.4. Sharing is important for all facets of the cultural and media scene, including books, photographs and new, Internet-native media. But it is the music and motion picture majors that have started a real war on sharing. ${ }^{32}$ Our purpose here is to list the possible reasons for their fierce opposition to sharing. The impact of sharing on sales of works cannot be the sole motivation, as numerous studies are showing that it is limited or nonexistent. It is thus reasonable to consider a range of other reasons:

- Cultural or ideological factors should not be underestimated. The industry has assumed for decades that its business rests on a degree of exclusive control over the production and dissemination of copies of works, so its reaction to the loss of this control is unsurprising, even though its profits are still healthy. In particular, large media firms find it hard to relinquish the very attractive prospect of producing and distributing copies of works for next to nothing, whilst retaining exclusive control over the process and charging monopoly prices. They have seen the shimmering mirage of Eldorado and are not ready to let go of it. 
- Just when more titles than ever were published on records and DVDs, the majors have chosen to restrict their offer: the number of music titles distributed by major companies has shrunk by a factor 4 or 5 at least. This approach has arguably been successful, in that they have maintained their profit per title, but it clearly fails for direct digital distribution (see below). As a result, large media firms are now trying to install new forms of control on digital distribution channels, and this is easier to implement in centralized distribution channels than in the context of decentralized sharing.

- Similarly, a key foundation of their present business models is their ability to concentrate the public's attention on a limited set of works. Major companies have been investing more and more in heavy promotion of a limited number of titles, with shorter and shorter individual lifetimes. This is clearly at loggerheads with the trends favored by sharing: increased diversity of attention and enlarged range of accessible works.

- Quite simply, they are afraid of the unknown. In truth, sharing has had only a limited effect on them so far. But they fear that if it was recognized legally, it would turn into a black hole that would swallow the creative economy whole.

After close to 15 years of this "war on sharing", the large media companies now know that it is here to stay. But they still hope that they can keep it clandestine, polluted, and stigmatized. They may be playing for time, trying to install some control over new channels before they have to live with sharing. In particular, it makes sense for them to try to push users back into a passive consumption mode. But although this passivity might be desirable for the cultural industries that flourished in the pre-digital era, it is not in the public's best interest, and policymakers should not necessarily embrace it.

When digital works are shared clandestinely, the resulting diversity of attention is lower than when sharing occurs in the open. ${ }^{33}$ This is because in a legally recognized context, one can rely on a degree of longevity and accumulation. Sharing rare works and, in return, obtaining others which one didn't have access to become credible propositions. When unauthorized sharing faces repression, sharers are led to prefer schemes providing a fast access to recent works, such as BitTorrent. However, even in such situations, attention is still less concentrated on a few works than in a central publishing model.

Despite many years of "war on piracy", unauthorized file sharing has already started to have positive effects on cultural diversity. At the 20Io MIDEM (a yearly music publishing business fair held in Cannes), SACEM, the French collecting society for authors and composers of music made an apparently mundane, but in fact very noteworthy statement (Lefeuvre 2010). The spokesperson for SACEM explained that the collected rights from digital sales remained very low, adding up to only $€ 6.5$ million for the year 2009 , but went on to mention a "long tail nightmare, with the four previous years resulting in 409 million sales spread over 2.6 
million titles". ${ }^{34}$ Let us start by addressing the first part of the statement. The weak development of commercial downloads, slower in Europe than in the US, may be attributed to many factors. The media publishing industry attribute it to the "unfair competition" of "piracy", despite evidence that file sharers buy at least as much digital music or video as people who abstain from sharing. ${ }^{35}$ The industry's critics see it as a sign of the rejection of outdated commercial models that fail to recognize user rights or to provide more than just access to a digital file.

\section{Delimiting the non-market sphere}

'Non-market' doesn't just mean not having to pay to access a piece of work. Access to a catalog following a subscription is not 'non-market', even if one does not have to carry out a monetary transaction to access each work. On the other hand, one might charge for the means to carry out certain activities without the latter losing their non-market nature. The case of contenthosting sites which are financed by advertising deserves a separate analysis: they are nominally used in a non-market way, but since they trade the attention time of their users with advertisers, for that part of their activity these sites should be considered as commercial distributors like any others.

'Non-market' doesn't mean administered. On the contrary, the development of non-market information-based activities represents a new step towards the realization of the efficient allocation of resources long promised by market economics. Markets, despite their value, are struggling to deliver this, due to their practical organization: unequal access to information and power, control over distribution channels, interdependence between products and technologies. Similarly, 'non-market' activities are not outside the economy. The supply of means to exchange information represents twice as large a fraction of gross domestic product (GDP) as the sale of information: see (UNU-MERIT 2006, pp. I23-I26). For more explanations of the value of non-market exchanges and other indirect means to fuel culture and other information-based activities, see The Wealth of Networks by Yochai Benkler (Benkler 2006).

Moving to the second part of the statement, the "long tail nightmare", which plagues SACEM and the majors, is good news for cultural diversity. It is testimony to the fact that it is more difficult to concentrate attention on a limited number of titles in the digital sphere than in physical distribution, at least when complementary channels such as file sharing exist. The 2009 annual report of SACEM claims that "one can only note the extreme concentration of sales on a few titles" mentioning that "on iTunes, only Io titles were sold more than 25,000 times, while the number of titles sold via download only was 20 million" (SACEM 2010, our 
translation)..$^{36}$ We will come back later to the issue of concentration of sales on iTunes, which is indeed strong in comparison to $\mathrm{P}_{2} \mathrm{P}$ file sharing. But the stated figures do not imply a stronger concentration than for record sales, quite the contrary: the key difference lies in the number of titles made available and the low level of average sales. What SACEM actually means is that few titles generate copyright revenues at levels which they can efficiently manage. Our thesis is that the increased diversity of attention can give rise to new resources to enable creative activity and a manageable and equitable distribution of funding and income. What remains to be seen is whether the collecting societies (where they exist) and the majors everywhere can adapt to this new world. Up to now, they have focused on preventing it from becoming a reality.

In the last few years, $\mathrm{P}_{2} \mathrm{P}$ sharing, whose protocols consider each individual as both a distributor and receiver, is said to have declined in favor of authorized or illegal streaming servers. Streaming services give access to a wide variety of contents, and thus few commentators have noted that this partial replacement of $\mathrm{P}_{2} \mathrm{P}$ networks by streaming servers is far from being good news. The business models of the operators of streaming services such as Deezer or Spotify are based on advertising, subscriptions, and content producers paying for the promotion of their contents. The fact that the 4 major phonographic companies have taken a participation in Spotify (Redwood 2010), while allowing it to provide access to their catalog, should act as a warning. This behavior can be seen as an effort to retain, in this new channel, the same strong control over which works reach the attention of the public that they have in classical publishing. ${ }^{37}$ Furthermore, if streaming becomes the dominant form of access to works, individuals would be turned into passive receivers.

If and when file sharing is recognized as a legitimate activity, it will become possible for users to choose technology and services based on their merits and properties, and not just because it is less risky to use one than the other. This would transform the current situation not because of the existence of file sharing, whose already massive scale would increase yet further, but because of the official legitimacy of exchange practices. It would result in a wider attention to creative works and a better recognition of their authors. The diversity of works able to reach a significant audience would vastly increase. The quality of the digital representation of shared works would be much improved. New services would emerge to support these exchanges. Creators and producers would compete to set up the most productive relationships between individuals and the other cornerstones of the creative economy, namely on-line artistic communities, services such as concerts, teaching or projection in theaters, or new forms of publishing on carriers such as collector sets and mixed-media publishing.

If exchanges of files containing creative works without specific authorization are useful, one may ask why we advocate recognizing only those that are non- 
market. There are two reasons for this, which we develop in the following chapters:

- the need to maximize the benefits that these exchanges yield (see box on the specific benefits of non-market exchanges in the information sphere above on page 28);

- the need to ensure that these exchanges co-exist as harmoniously as possible with other cultural activities, particularly those which lead to monetary transactions and help fund creative activities. 



\section{Sustainable resources for creative activities}

There exists a caricatural view of the Internet, which prevents a constructive reflection on financial resources for creative activities and culture. Those who adopt this view see the development of on-line non-market sharing as a black hole that would swallow up the cultural economy whole, and with it culture itself. They also imagine that the Internet could become an Eldorado for new cultural industries to flourish in, as long as the scarcity of copies of works which is the rule in the realm of physical carriers would also be enforced in the digital sphere. Ms. Christine Albanel, the former French Minister for Culture, expressed this view in its purest form in the explanatory memorandum for her 2008 "Internet \& Creation" bill: It is now possible to turn digital networks, for the benefit of consumers, into a true dematerialized goods distribution tool, particularly in the cultural arena. This will only be possible, however, if intellectual property rights are respected. Yet, at the same time, the conditions for the creation of these works have never been more threatened. In 2006, billions of pirated files containing musical and audiovisual works were exchanged in France. ${ }^{\mathrm{I}}$ We address this view in further detail in Chapter II.

The reader will by now have understood that we view the notion of maintaining the scarcity of copies as not only impossible, but downright harmful, as it seeks to dispossess individuals of the capabilities which are crucial for the construction of a shared culture. As for the black hole scenario, it is factually erroneous: whatever negative impact results from the development of non-market sharing will be limited, and will affect only certain forms of cultural economic activities and sources of income for authors, whilst others will be affected positively. Before detailing how we would propose to finance a file sharing compatible creative ecosystem, let us briefly take stock of the state of fact-finding on the impact of file sharing. This will clarify further why we do not view it as a problem to be removed, but as a vital part of the creative economy of the future.

Of the commentators who are still proclaiming that cultural creativity is being bled dry, most use music as their preferred example. However, independent research studies focused on music show that increased sharing does not decrease the income from a given work (Oberholzer-Strumpf 2007, OberholzerStrumpf 2010) or decreases it only to a limited ${ }^{2}$ and varying degree (AndersenFrenz 2008). The market for recorded music has indeed shrunk, however, a fact that other studies impute to file sharing (Liebowitz 05). This apparent contradic- 
tion probably arises from a change in the supply pattern. Major publishers have reacted to their inability to enforce their exclusivity on the distribution of digital works by restricting supply and focusing marketing on a small number of titles. This has so far enabled them to maintain and even increase per-work profits, but at the cost of a reduction in the overall size of the market and of the resulting income for all but a few artists. This reduction is partly compensated by the growing supply from independent producers and individuals, but their activity remains fragile as long as they stay dependent on centralized distribution and promotion channels. Despite this, the overall economy of music has never stopped growing, if one considers not just the market for recorded music, but also concerts, teaching and instruments (see below section 7.4).

Studies regarding film and audiovisual material (Marsouin 2008, Martikainen 2010) lead to the same conclusion: non-market file sharing has an excellent synergy with theater ticket sales, and even sales of DVD or commercial downloads are affected little, if at all, by non-market sharing.

Judges themselves have often expressed their skepticism towards the claims of publishers, according to whom any unauthorized exchanges imply lost profits. That is one of the reasons why the corresponding lobbies pushed for the AntiCounterfeiting Trade Agreement - whose ratification is raising a lot of debate at the time of writing - to rubber stamp their viewpoint and dispense them from having to prove their losses (KEI 2008).

The debate will no doubt go on, but on the basis of presently available evidence, our primary reason for creating new financing sources for creative activities is not the supposedly adverse economic impact of non-market file sharing. There is another, much more compelling reason to seek new resources to finance creative activities, and that is to take advantage of an historical opportunity. Sharing opens the perspective of a many-to-all cultural society, in which everyone has access to creative, expressive and informative works and has the means to contribute to their creation. In such a society, many will produce works that deserve the attention of some, and quite a few - many more than today - will obtain the attention of many. We call it a many-to-all cultural society because it transforms the previously stark distinction between producers and consumers - those who create works and those who access them - into a continuum. It puts them all into a position to benefit from the cultural commons, provided that the process can be sustained via proper reward and financing mechanisms.

The transition to a many-to-all cultural society raises great challenges. In the information era, cultural practices are growing at an unprecedented rate, precisely as a result of the fact that they are, for the most part, non-market. These new activities cannot be financed only by traditional means. This applies in particular to capability building: if many of us are to take part in new creative activities and produce output that is of interest to others, we need time and money to become better at it. Financing mechanisms will serve their purpose only if they can 
be put in place without creating excessive transaction costs for users, and without restricting the very freedoms which enable the activities in question to take place. In addition, we have to finance a much wider domain of creative activity, whilst the contribution of some of the existing sources (television, sales of physical carriers) is bound to decrease. ${ }^{3}$ We must thus devise new financing means, able to grow with cultural and artistic activities and to contribute to the recognition of quality among their products. The question is: how?

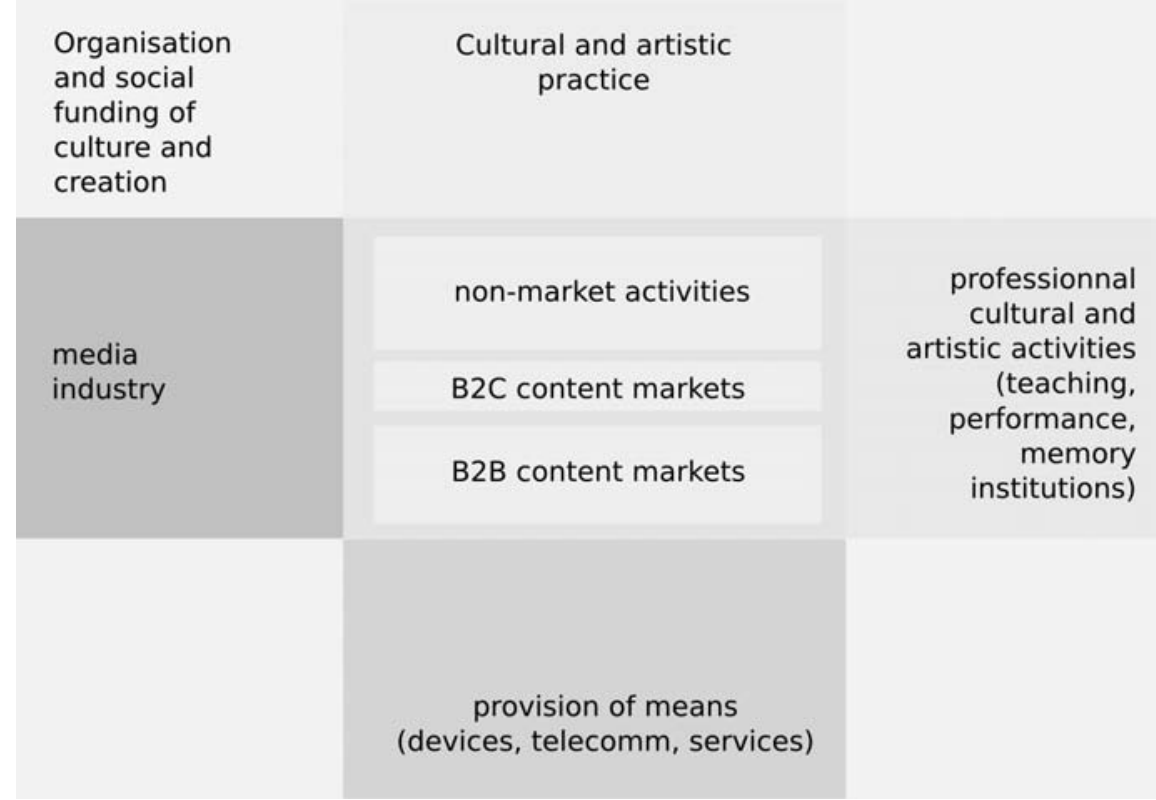

Fig. 4.1. Cultural and creative activities and their economy.

We must first define the range of activities concerned, as different stakeholders tend to place emphasis on different components of culture and creative activities. The debate on the financing of creative activities has mostly arisen in the context of a very narrow vision, focused on the private consumption of the products of the cultural industry, the economic performances of the companies producing or distributing these products, and to a lesser extent the royalties collected by collecting societies. Figure 4.I sets these domains in much wider context, enabling us to take stock of the challenges facing us.

Let us dispel a few widely held notions. Of the copyright revenues and associated royalties distributed by publishers or collecting societies to those who contribute to creative activities, only a very small fraction - except for books - arises from the private consumption of cultural goods, whether physical or digital (the 
Business-to-Consumers or $\mathrm{B} 2 \mathrm{C}$ part of figure 4.I). Similarly, the economy of cultural goods represents only a small part of the artistic and cultural economy, which is in fact dominated by the support of non-market activities (UNU-MERIT 2006, pp. I23-126) and the provision of services, whether for profit or not (education, live performance, theater projection, ...). (Benhamou-Sagot-Duvauroux 2007) showed that artists draw only a relatively minor and rapidly decreasing portion of their income from copyright. More generally, the fraction of artists' income arising from the sale of cultural goods such as musical recordings is decreasing in domains such as music (SACEM 2010).

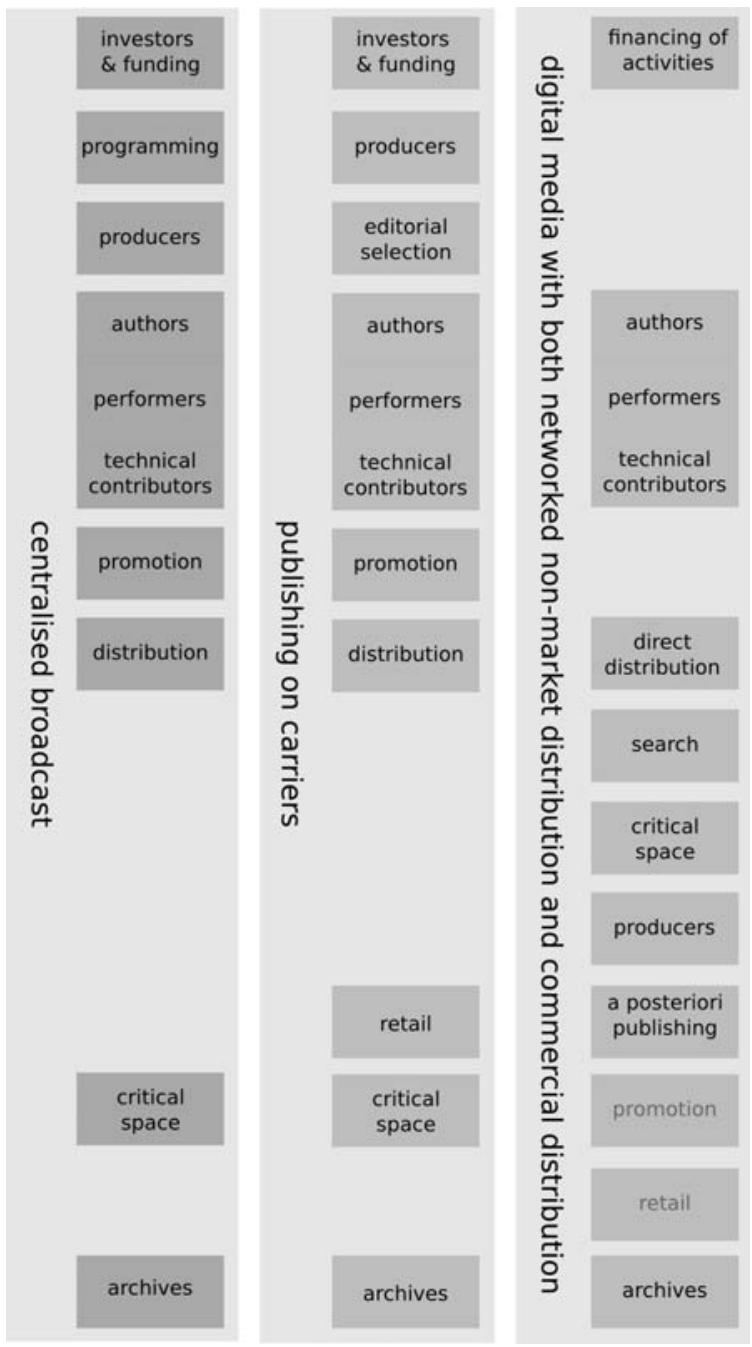

Fig. 4.2. Roles and functions in media. 
These remarks should not lead us to disregard the very complex chains of activities that are necessary for the health of a creative ecosystem. As shown in figure 4.2 , a creative economy developing in a context of widespread sharing of digital works is not free of intermediaries, quite the contrary. Here, the third column presents an extreme scenario, where authors and artists distribute their works directly for sharing on the Internet, and commercial players either serve this activity or do ex-post publishing. We do not believe this will be the unique scenario, but it is already very much present and helps us to reflect on the functions that are needed in the digital creative ecosystem. Many of the traditional added-value functions that exist in classical publishing or broadcast are still needed, though they take place at different stages. New functions are also needed, to perform searches for example. Others now have to be practiced in considerably more challenging situations: the detection of valuable works, their editorial selection in view of publishing, the critical space without which there is no cultural scene must all address considerably larger sets of works.

So we have great opportunities and great challenges. To address them, we explore a wider range of solutions to promoting a flourishing, sharing-compatible cultural economy. Existing schemes such as market sales of goods and services, copyright revenues from commercial reuse, or public subsidies ${ }^{4}$ will remain essential. New schemes such as voluntary resource pooling between individuals to finance specific projects are emerging, and they are proving relevant to address some financing challenges in digital media, for instance for investigative reporting or documentary movie production. However, the degree to which these schemes can scale up is somewhat unclear. This led us to seek solutions that directly tackle the scale of financing needs in a many-to-all cultural society. In the rest of this book, we analyze the needs and potential of large scale mutualization organized by law. The term mutualization (organized pooling of resources between parties having a common interest) is not frequently used in English, where it designates mostly the process by which a stock exchange company turns itself in a co-operative. In the Igth century, mutualism grew - in particular in France and Italy - as a political and social movement, organizing co-operatives in all forms of production, commerce and social services. It promoted contributory schemes to pool resources in domains where everyone is a producer and a user of products and services. Mutualism competed with socialism and has a renewed relevance in the Internet age. We consider in this book, in addition to voluntary resource pooling, a mutualized financing scheme for cultural and expressive activity, based on a statutory (compulsory) financial contribution. We call this scheme Creative Contribution.

Even for readers who do not view the idea of a compulsory contribution for financing the cultural commons as necessary or relevant, most of what is developed in the following chapters should be of interest nonetheless. These chapters contain: a discussion of the rights that are essential for all participants in culture; 
a detailed modeling of the rewards to creators of valuable cultural products; an analysis of the global needs for new creative projects; proposed designs for measurement systems which ensure the fair reward of contributors without intruding into the privacy of users; and a discussion of democratic governance for largescale mutualized financing systems. All of these topics are relevant to any system that would address the sustainability challenges of digital culture.

Nonetheless, statutory contribution schemes such as the creative contribution have specific advantages, which are why they are currently emerging as a key outcome of close to ten years of debates on financing mechanisms associated with the right to non-market sharing between individuals. In the last few years, they have been discussed in many circles, from parliaments to free culture communities, from artist collectives to Internet freedom advocacy groups. These debates helped identify some key questions which needed to be explored further: ${ }^{5}$ Who should contribute? To which parts of our 'galaxy' of creative and economic activities should users be expected to contribute? Where should mutualization stop, lest it become a kind of opportunistic tax, collected wherever possible, without clearly laid out rights for all? The framework we propose in chapters 5 to 8 answers these questions in detail, but let us lay down the fundamentals of this response straight away: ${ }^{6}$

I. Everyone who has access to broadband Internet benefits, or will benefit, from the digital cultural commons, of which sharing is a key enabler. It is thus legitimate to ask everyone to contribute to the existence of the works that populate these commons, to their quality and to the recognition of this quality. This contribution must come with cleared stated rights, and each contributor must have effective powers to choose how this contribution will be used.

2. The first aim of this contribution is to reward creators and other contributors $^{7}$ to works. In practice, it will more than compensate for any possible impact that the recognition of a right to share may have on their other sources of income, but the primary motivation of this contribution is not compensatory: it is based on newly formulated social rights of all participants to the cultural commons.

3. It is also legitimate to ask the users of an Internet where non-market sharing is free to contribute to the production of digital works whose creation requires upstream investment. However, this contribution must bear a relation to the extent to which these works are targeted to or used on the Internet. It is also important for Internet users to contribute to types of creation which are not easy to realize in a market context, such as personal artistic projects, investigative reporting or photo-reporting. The allocation of funds for support to production can be entirely based on contributor preferences. 
4. Finally, it is also important to support an environment where interesting works can be detected and recognized. This calls for numerous actors and functionality enabling the users to find, recognize, and construct quality in a sea of works. Some of this functionality is presently sustained or can be sustained via subscriptions, donations or indirect advertising revenue. Others require a more global mutualized financing to be sustainable.

To conclude the introductory part of this book, we should recall that implementing a mutualized (societal) financing mechanism for creative activities, in response to a new technological situation, would not in fact represent such a break with the past. Since culture has been considered as a specific domain, creative activities and their authors have been financed via a combination of mechanisms. Indirect mechanisms (sponsorship, public funding of certain statuses and activities, fiscal incentives, or mutualized financing via fees, levies and donations) have always dominated over the direct sale of cultural goods. The advantage of new forms of mutualized financing is that, thanks to information technology, they take place in a cultural society of peers. They create additional links between creators, their works and their users, without necessitating intrusive surveillance of individual usage.

What should we reasonably expect from a mutualized financing such as the creative contribution?

- a strong increase in actual cultural diversity: see figure 3.2;

- a real reward arising from digital non-market usage, for a vast number of creators and contributors, but mostly at the level of a supplementary rather than primary income;

- a contribution to mechanisms which help produce and maintain quality in various media, including emergent media: see section $7 \cdot 3$.

What should we not expect from it?

- that it should act as the sole source of income for all creators, or finance an entire production chain;

- that it should save commercial models which never had any credibility, such as the large-scale on-line sale of digital cultural products at monopolistic prices without added-value functionality (see entry I2 in the FAQ of chapter II). 
We will now consider a key decision: on which foundations should we build the new proposed mechanisms? Should we consider them as the organization of the cultural and media market? As a particular form of copyright law implementation reforming it from the inside? Or as a new social pact based on new formulations of rights? Of course, the answer will not be one or the other, but will include some aspects of each. What we recommend in the next chapter is a new balance between these three approaches. 
The Creative Contribution 



\section{Which rights for whom? A choice of models}

A number of models have been proposed over the past few years to move beyond the repression of file sharing. In this chapter, we analyze them and complement them with our own proposal. We discuss the benefits and drawbacks of the various models, and obstacles which must be overcome before they can be implemented in reality.

A major distinction between different models is which rights they give to whom. Do they give actual usage rights to individuals? On which basis do they plan to reward creators? Starting with models that do not recognize the right of individuals to share contents, analyzing some limits of copyright-based and other compensatory approaches to the legalization of sharing, we will progressively sketch out the key characteristics of a new model, based on social rights. Our goal is to formulate a model that can provide a perennial source of finance to a creative economy where non-market sharing of digital works between individuals is recognized as legitimate.

\subsection{Access without rights to share: commercial download or streaming licenses}

The media industry dilemma

In 1997, we published an article in First Monday, a peer-reviewed on-line journal on Internet research. Attention, Media, Value and Economics (Aigrain 1997) was a contribution to a debate on the "attention economy" launched a few months earlier by (Goldhaber 1997) and (Ghosh 1997). Based on earlier work (Aigrain 1996), the paper analyzed how much people are willing to pay for one hour of interaction with music or video, for instance. The answer depends on the circumstances and the form of interaction. Unsurprisingly, in the cultural domain, people are ready to pay considerably more per hour if they experience the uniqueness of a live performance, ${ }^{\mathrm{I}}$ or even when an event merely establishes a relationship with other members of an audience (for instance the projection of a movie in a theater). This live experience does not apply to recorded contents or broadcasts, but there are still great differences between forms of access and usage. What matters then is what people can do with contents. To date, people still pay more 
for recorded music or video than for radio and TV, even accounting for indirect payment in the form of advertising. ${ }^{2}$ This is because owning recorded contents allows the user to carry out a number of actions, not least copying. In fact, recent history shows that users simply won't buy contents if they are prevented from copying them, as demonstrated by the strong rejection by consumers of copyprotected CDs and digital contents with technical protection measures that are not easily circumvented. ${ }^{3}$

This evidence has faced the media publishing and distribution industry for close to 15 years. In the digital world, it must make a choice. Either the industry reinvents itself, to become a producer of quality contents, facilitating fan-artist or fan-fan relations, and helping people to find contents they like, or it tries to remain a gatekeeper, selling access to copies of works, or renting usage rights at a monopoly price.

Not all media companies pursue only the latter option. The Hollywood film industry, for instance, maintains a strong investment in high-budget movies (such as Lord of the Rings, Avatar, etc...), on the premise that these extremely expensive movies will become premium contents that everyone wants to see, because of their quality or, ironically, because of their fabulous cost. It is also exploring new ways to derive income from merchandising or product placement. Similarly, TV series producers have understood that on-line video sites originally designed for user-generated contents could become an alternative distribution channel for TV, compensating for the loss of TV viewing time. They did not frontally oppose the copying of their contents, but instead threatened the operators of these sites with lawsuits in an attempt to force them to negotiate commercial agreements. ${ }^{4}$

On the other hand, the music industry majors originally clung to the mirage of retaining their oligopoly on the sale of musical recordings, or on the licensing of listening rights to consumers, an even more attractive game where people pay each time they listen. This is only partly successful, however: people do pay for contents, but preferably when there is no exclusive control on copies, i.e. no digital rights management systems to prevent them from copying them or conducting other unauthorized acts. They use streaming, either on unauthorized sites or on sites funded by advertising or subscriptions, whose long-term sustainability remains uncertain.

Despite having started off in different directions, the audiovisual and music industry are gradually converging towards the same basic ideas. The general intention is to offer users access to a wide range of works, without giving them the right to share it with others. Can the Internet become a giant on-demand TV system? The appeal of such a solution for media companies is double: they might derive some income from selling the right to give access to contents to intermediaries, but more importantly, they retain the ability to concentrate attention on a few works by controlling a limited number of distribution channels on which 
promotion and advertising can be targeted. This is their deeper reason for opposing sharing: when hundreds of millions of individuals have the right to share, they become a distribution medium of their own. As we have seen in chapter 3, some works still receive much more attention than others, but much less so. Worst of all for the large media industry, it is harder to predict which ones will.

\section{Oligopoly}

The term oligopoly refers to a small group of companies that, together, control the supply of certain goods and are thus able to determine which are made available to the public and at what price. Oligopolies currently exist in the record industry, where over $75 \%$ of publishing and $90 \%$ of distribution are controlled by 4 multinationals. Oligopolies also exist for motion picture production in the US and for distribution to theaters in many countries. For concerts, there is a quasi-monopoly of LiveNation for the global concert tours. In France, although there is a quasi-monopoly in the book industry, the fact that some publishing brands remain independent in defining their editorial policy and the survival - even if fragile - of independent bookshops, ensure a reasonably diverse supply of books. For consolebased computer games, oligopolistic control applies more to the consoles themselves than to the content. In all areas, this control is now strongly challenged in the digital networked world. However, attempts to centralize on-line access and marketing are now starting to emerge, which may result in a new form of control on the supply of works to the public. For instance, Apple holds a strong position in the distribution of digital recordings with iTunes, reinforced by the fact that Apple also manufactures devices and runs proprietary application stores (AppStores). Apple tries to leverage this position into other media, while other players try to emulate Apple, for instance, Amazon with its Kindle eBook reader. Google tries to use its dominant position in search engines into other domains. It has a more open approach to devices (the Android platform for mobile devices) and AppStores than other players, but the evolution of its contents-related sites and services (YouTube, GoogleBooks) and the exclusive agreements signed with publishers and libraries have raised concerns. Preventing the potential of the Internet from being hijacked by a limited set of interests is one of the challenges of the present debate.

\section{Flavors}

The core component of this "access without sharing" model is the granting of commercial licenses to intermediaries, allowing them - and only them - to offer 
contents for streaming or download. People may access works free-of-charge on advertising-funded sites, or may have to make unit or subscription payments, but in all cases the exchange of contents between users is prohibited. This idea comes in many flavors, which we briefly describe below because they illustrate the power play between media corporations, telecoms companies, new intermediaries, and collecting societies where they exist. We designate the various flavors according to the relationship between various types of players: vertical integration, distributor monopoly, right holders cartel, and collective management under a legal license.

Vertical integration is already on the market. The idea is simple, and consumers in many countries will have seen it advertised in various forms: in 2009 it was "buy a Nokia phone, a subscription to your preferred mobile communication operator, and get access for one year to (most of) the Universal Music catalog". As all mobile operators and mobile phone suppliers would like to be on board, the majors organize an auction to decide who will get their catalog. This leads to a segmented contents market, where people have to choose which catalog they will have access to. It also causes the contents owned by the majors, particularly the leading one, to receive a preferential treatment. It would be truly appalling if it worked, but fortunately the limited success of these offers shows that consumers do not accept being restricted to one content-provider catalog.

Distributor monopoly is a more successful model, because it provides a wider access, though with an even stronger integration of power in the hands of one player: buy an iPhone or iPad, and get access to its manufacturer-controlled list of authorized applications for access to contents, as well as a subscription to the mobile phone company selected by the same manufacturer in your country. ${ }^{5}$ This has better chances of success, because the control is less visible, and is in the hands of a player that offers something real (a device), and on the surface appears to allow certain acts (such as developing and sharing applications and content) ... until it decides that your preferred content is bad for its image. The true strength of this model is that it professes to limit sharing, but actually does not care as long as the sharing will mostly occur within its realm of operation. ${ }^{6}$ This approach offers more value, but at the cost of a locking-in effect: it creates a large club of dependent customers. These consumers provisionally ignore the boundaries of the realm in which they are confined.

As mentioned above, ${ }^{7}$ in 2009 , the 4 musical majors took a participation in the Swedish company Spotify, a significant provider of streamed music. At the same time, they jointly gave a license for streaming of most of their catalogs to Spotify in a then unexpected gesture. As a result, the value of their share in Spotify increased significantly. When rumors surged in April $201 \mathrm{I}$ that Google was interested in buying Spotify, its value was estimated at \$r,ooo million, representing a giant added value (vanBuskirk 20II). It is worth noting that the majors would not have to distribute a cent of this added value to creators, as it does not constitute 
licensing income. Meanwhile, their share in the capital gives them power to promote their own contents, which of course will benefit creators ... or at least some of them. However, this model is limited in economic terms: users like streaming as long as it is a free radio, but will pay little for the limited value it delivers. The reader will find an interesting discussion about this value (or lack of) in (Hillage 2009): in answer to the manager of a record label, who complains of having received only $\$ 6.70$ for 33,983 stream plays of their contents, a commentator points out that this is probably as much as the income one would expect from one broadcast on a radio with this number of listeners. Even if they do not resell their participation, and despite the uncertainty on the ultimate success of Spotify's business model that is increasingly based on paying subscriptions, it is far from obvious that the major companies will come to regret their investment: keeping control of distribution channels remains their main concern.

\section{Methodological note on currencies}

In the rest of this book, and in particular in chapter 7, we discuss macroand micro-economic figures and various financing schemes for creative activities on the Internet. Most of these figures come from statistical aggregates whose latest available value can range from 2004 to 2010. During this period, the Euro to US dollar exchange rate has fluctuated widely, between I.I8 and I.58. These fluctuations were driven by the currency markets and are not explained by related changes in purchasing power parities (PPS). To avoid confusing the reader, all statistical aggregates are reported in local currency, that is, for the key cases under study, in Euro for France and Germany and in US dollars for the US. The sign \$ should always be interpreted as US \$. Some figures are design choices in our proposals, for instance the minimum amount which we consider a "significant" reward for a contributor to works. To allow for comparisons between the various countries under study, we have set these figures using a somewhat arbitrary currency rate of $\$ 4=€_{3}$ corresponding more or less to the average rate over the last 5 years.

The Music Match agreement between Apple and two music majors, announced in 20II, signals another possible merger between vertical integration and the dominant position of a distributor. Under this agreement, Universal Music and EMI accepted an offer from Apple to allow it to offer, for $\$ 25$ per year, subscriptions to a service whose users can obtain high quality copies of any piece of music that is on their hard drives, whatever its origin. Commentators have described this service as a form of "piracy laundering" where users could obtain a legal copy of files originally downloaded without authorization, and they expressed surprise at the fact that the majors agreed to this. However such a scheme is indeed profit- 
able to both parties: it creates a combined monopoly of contents, distribution and devices. The net result, if such a scheme is allowed to proceed, would be to limit the ability of creative works, services and devices that are not included in the deal to reach consumers or obtain revenues. In all logic, such a scheme should be even less able to withstand legal scrutiny than the GoogleBook settlement (EfratyTrachtenberg 20II).

Finally, we turn to the collective management flavor. In this scheme, a law creates a compulsory collective license to offer contents for streaming or download, but without the right to share. This means that the operator of a streaming or download platform does not have to negotiate for the permission to provide works. Either this license would be obtained by Internet Service Providers against payment of a flat-rate per user, or it would follow the statutory licensing scheme that exists for radio in many countries: any intermediary would know in advance that it will cost so much per play or per download. ${ }^{8}$ The flat-rate approach was advocated for music by Laurent Petitgirard, former President of SACEM, the collecting society for music authors and composers in France. ${ }^{9}$ It received little endorsement: rights holders did not like it because it created a preferential system for ISPs while it did not please advocates of the right to share because it did not grant that right.

The Zelnik Committee, created in 2009 by the French government and chaired by the head of the Naïve record label and president of the IMPALA European lobby group of record labels, advocated a compulsory music streaming or download license for commercial offers by ISPs or other intermediaries. Large independent record labels like this approach because they fear that, without it, their contents will not be represented on streaming and download platforms, the latter using all their money to obtain the most visible contents from the majors. In fact, if this approach is applied to all media and associated with the recognition of non-market sharing, it would be an excellent idea, avoiding segmentation in the commercial market. But if it is implemented in the context of a continued war on sharing, it will have only a limited benefit. The ability of the majors to concentrate attention on a small number of titles will be weakened to a certain degree because suppliers are guaranteed a theoretical access to all titles, but attention will remain much more concentrated than if sharing were recognized. A more serious worry is that the major corporations would have sufficient lobbying power to enforce a high licensing cost per download or per play. The value delivered without right to share would remain low. As a result, only the largest intermediaries (such as Google or some telecommunication companies), who can afford huge investments to capture the attention of people, would be in a position to license a large number of titles. The major flaw of licensing contents to ISPs or other intermediaries, instead of recognizing sharing rights to individuals, is that it gives an extraordinary amount of power to the licensees. In particular, the risk is that they will 
be able to emulate large distributors of consumer goods, selling products to consumers and product placement to content providers.

As described above, the value of access to digital contents is highly dependent on what one can do with it. By offering access but restricting the most elementary actions - copying and transmitting to other individuals - one limits the value of the offer to users, and consequently their willingness to pay. This does not mean that access to contents has no value in itself, it simply reflects the fact that it is not a scarce resource in the digital world. However, while insisting that it is access which one should pay for, the media industry does nothing towards the provision of true added-value services, such as helping people to locate what they like in an ocean of contents, facilitating the relationship between artists and the public or between fans, or adding value directly to the experience of listening, viewing and re-using.

Legal licensing for commercial offers also faces a number of legal and policymaking challenges. It would necessitate the creation of a new exception to the exclusive right to make works available to the public, ${ }^{\mathrm{IO}}$ and hence a modification of the European legal framework (see also page 74). The media industry will also object strenuously if it is not allowed to implement differential pricing for its premium contents, although one can argue the large number of unit payments for plays or downloads, each of which comes at no extra cost to the right holders, should be more than sufficient to reward the "premium quality" of these contents. If a political will to implement legal licensing for commercial offers exists, these challenges are not impossible to address, and it can be a useful complement, provided that the right to share is recognized.

If the non-market sharing between individuals is recognized as a legitimate activity, and we acknowledge the need for new means of financing creative activities, on which basis should we design the latter? How much of the collected sums should be used to reward existing works, and how much to finance the production of new works? Should the reward mechanisms be thought of as compensation for a form of harm, as copyright licensing, as remuneration for work done, or as a reward for a socially useful achievement? These questions are debated in a context of great confusion, because they touch on a complex mix of legal, economic and policy issues. We propose to distinguish between two main approaches: compensation schemes on one side, and social rights to economic and cultural benefits on the other side.

\subsection{Compensation schemes}

One of the motivations of the initial proposals (Von Lohmann 2003, Alliance 2005) for a flat-rate contribution by Internet users to reward the creators of works shared over the Internet was to stop the "war on piracy". The fight against file sharing launched by the large media industry and, in Europe, collecting socie- 
ties was leading to ever more surveillance, ever more repression, and a climate of legal uncertainty for individual users and providers of technology. It threatened the very existence of the Internet as an open space for the exchange of information. It seemed as though personal information technology platforms would soon cease to be under the control of the person owning them. The idea of "buying peace" by compensating creators and distributors was not stated openly, but it was on the minds of many.

There are various legal bases for a compensation-based reward: for example, it could take the form of licensing of copyright for specific activities, or of liability compensation for the inability to enforce an existing exclusive right. The home copying laws for phonorecords and video recordings are often described as liability compensation. The existing laws on book photocopying in France license the reproduction right to a collecting society that can enter into agreements with users, while setting that the collected sums will be distributed half to the publishers and half to authors. ${ }^{\text {II }}$ Most proposals for the legalization of file sharing so far have used this licensing approach, and we initially followed the same path (Aigrain 2008).

William Fisher proposed another approach, recognizing that copyright, in the strict sense of control over copying, cannot be enforced in the digital world. He proposed to put in place "government-administered rewards" for works shared on the Internet. However, the overall amount of these rewards is still designed to reflect a compensation. In chapter 6 of his book Promises to Keep, William Fisher outlines various possible ways of defining a system to reward the usage of works on the Internet. He expresses doubts about the possibility of defining a fair reward directly, and suggests that "we could use the new reward system to compensate creators and their assignees for the losses they have suffered - and will likely suffer in the immediate future - as a result of being deprived of their ability to enforce their copyrights in the new technological environment". He then goes on to specify: “... it could seems wisest, when replacing the current copyright system with a system of government rewards, to begin by holding more-or-less constant the aggregate amount by which creators are currently compensated..."

There are pros and cons to each type of compensation scheme. Liability torts simply compensate right holders for their inability to enforce their exclusive right in the private sphere. They do not create a right for individuals to share works, but only a tolerance. Rights holders are likely to interpret this as being subject to review each time a new surveillance or control technology suggests that copyright could become enforceable in the digital sphere. Copyright licensing to individuals does grant users a true usage right, but it opens the door to a permanent renegotiation of the rate at which it is licensed. Government-administered rewards bypass this problem by moving the management of rewards out of the copyright system ... once they are established. However, given that their amount is based on compensation, there is a risk that their introduction would give rise to an ava- 
lanche of compensation claims. Furthermore, as we will see below, a compensation-based reward system is intrinsically unable to handle Internet-native media and to reward works which their creators share on a voluntarily basis.

Recently, developments in our knowledge of the creative economy and debates among the promoters of alternative reward systems have led us to consider models which depart from compensatory approaches. In a sense, our proposal is a natural successor to William Fisher's, one step further removed from copyrightbased reasoning.

Several years after the publication of his pioneering book in 2004, our understanding of the effects of file sharing on various sources of revenue for creative activities has progressed significantly, though it remains imperfect. In the intervening years, file sharing and other forms of unauthorized access or exchange have flourished, leading the Recording Industry Association of America (RIAA) to multiply copyright infringement suits and damage claims against file sharers. ${ }^{12} \mathrm{~A}$ macroeconomic examination of the revenues of creators and media between 2004 and 2007 must go beyond the copyright economy, because copyright licensing and usage right sales (based on copyright) account for a small part of the overall cultural, artistic and entertainment economy. Of course, other sources of revenue, without taking the form of copyright royalties, may depend on the existence and enforcement of copyright. However, while the media and related industries cry out that file sharing (so-called "piracy") is devastating them, this is simply not true if one looks at the overall bottom line. All indicators are green, except for the investment of the musical majors in publishing new titles. In the US, the overall personal consumption expenditures for recreation (including all cultural or media products, plus sports and amusements, but not information services) grew by $44 \%$ from $\$ 586$, 000 million in 2000 to $\$ 84$ r,ooo million in $2007 .{ }^{13}$ The motion picture and video industries, which constitute one of the most vocal copyright interest groups, saw their turnover grow from $\$ 72,000$ million from 2004 to $\$ 82,000$ million in 2007 .

The music industry, however, is often said to be suffering. Certainly, in this era of strong unauthorized file sharing and transition to carrier-less digital recordings, the sales of musical records grew "only" from $\$ 9,800$ million to $\$ \mathrm{II}, \mathrm{I00}$ million, over the same period. Let us take an honest and detailed look at consumer usage and spending: from 2004 to 2007 , the average time spent listening to recorded music in one year went down from 199 to 177 hours, an $11 \%$ decrease. The average spending per person in the year went down from $\$ 52$ to $\$ 44.7$, a I4\% decrease. How can these apparently contradictory figures be reconciled? Exports did well, and the population increased, whilst the shrinking production of the majors led to a decreased per capita consumption. If more evidence as to the good health of music creation is needed, the combined revenue of recording studios went up by $25 \%$ from 2004 to 2007 . These findings are confirmed by Felix Oberholzer-Gee and Koleman Strumpf (Oberholzer-Strumpf 2010). Furthermore, their 
paper demonstrates that network-based file sharing never stopped increasing in the US and Europe, and that its impact on sales of musical recordings is at most responsible for $20 \%$ of the decrease of sales, which is much less than was assumed in the proposals for compensatory mechanisms made in 2003-2004.

Is everything fine, then? Not quite. Because the majors concentrate so much of the publishing and promotion power, the ability of the growing number of artists who distribute their works on-line to generate the income which they deserve from it did not progress, and probably regressed.

In 2008, researchers conducted a survey (Bacache et al. 2009) in which they interviewed 4000 members of ADAMI, the collecting society for lead musical performers in France. ADAMI members are relatively established artists - almost 3/4 of respondents were older than 40 - and are not representative of the much larger group of young emerging artists, support performers and quality practitioners. However, most of them are far from earning high incomes: only around 20\% declared having a net income (from all activities) of more than $€ 30,000$ per year! Additionally, 50\% earned half of their income or more from non-musical activity. More than 3/4 of those surveyed declared that the Internet (including file sharing) had has a positive effect on their notoriety. Some $45 \%$ estimated that the Internet had a positive effect on their concert audiences, but only $25 \%$ that it increased revenues from live performance, and $15 \%$ that the effect was positive on recorded music sales. As with any survey, these answers must be assessed with caution. However, they confirm that we have a situation in which the large media industry is doing fine ${ }^{\mathrm{I} 4}$ but performers, except for a few, are not. A similar situation is observed in the US by a Pew study (Madden 2004), quoted in (OberholzerStrumpf 2010): three-quarters of a large sample of interviewed musicians declared having a non-music complementary job.

Music performers have always been less well treated than composers of music and authors of lyrics. Nonetheless, similar trends seem to be affecting composers and authors. The vast majority of music author rights in France are collected by the SACEM collecting society. Between 2004 and 2007, the total collected rights rose from $€ 725.5$ million to $€ 759$.I million, ${ }^{15}$ though this amount has reached a plateau since 2005, which translates into a slight decrease when accounting for inflation. Phonographic rights (royalties collected from the sales of musical recordings) have decreased significantly from their peak value of $€ \mathrm{I} 42$ million in 2003 to $€ 89$ million in 2009, but this was compensated by other sources of income (live performance, television, reuse, and public performance of music, in particular). SACEM's annual reports provide no information on the extent to which the income it transfers to authors is concentrated on a few, or more equally distributed. However, in a 2005 interview (Axel 2005), SACEM's president stated that only 3000 members, i.e. $8.8 \%$ of those who collected rights from SACEM in that year, and $3-4 \%$ of all members, received more than the minimal wage. He did not specify how many were living artists: heirs of deceased artists receive an im- 
portant share of rights, since author rights in France last 70 years after the death of the last author (wartime not withstanding).

Given this, we should be wary of propagating the status quo into a new model, and thereby locking ourselves into it. Clearly, it was not the intention of the 20032005 proponents of alternative reward systems. They were convinced, as we are, that the legalization of sharing would generate more diversity of attention and a more equitable distribution of income, particularly if it was complemented by adequate competition in various markets such as live performance tours, distribution to movie theaters, sales platforms and retail distribution of goods. A compensatory reward would indisputably be a positive way out of the war against file sharing, but it risks building an excessive bias into the reward models of the future. As we will see below, copyright law is an inescapable part of the policy landscape, and we cannot afford to ignore it, but this does not mean that copyright law or copyright economics are necessarily the model of choice for defining adequate rewards. It is at least worth exploring another approach, which is to define the reward directly based on other considerations, and then to check if it is compatible with copyright law, or can be made so.

Another drawback of compensatory approaches to alternative reward systems, which is even more important in our opinion, is that they are unable to handle new IT and Internet-native media in a satisfactory manner. It is not necessarily easy, but for these new media, the only valid way to design a reward system is to start from first principles, i.e. to seek distributive justice. We lack any pre-existing reference, business or rights distribution model, which we could use to determine how much revenue, if any, is likely to be lost in the event that the right to share is recognized. Of course, new commercial models are defined every day in this digitally native world. Most of them struggle to reach any form of true sustainability. For a wide part of the open Web, this is not a problem: Wikipedia or most personal blogs will keep being produced without further financing mechanisms. But art, investigative or knowledge projects (including some personal blogs), where an investment beyond the individual's free time is needed to produce higher quality outputs or achieve specific results, need new forms of financing. The intermediaries (such as collaborative news media), which provide the environment in which capability building occurs, desperately need new sources of financing to become sustainable. For every success story such as Slashdot ${ }^{\mathrm{I}}{ }^{6}$ there are dozens of deserving collaborative media, free culture or Creative Commons (CC)-based projects which remain confined to niche activities, even though they are the laboratories of our cultural future. In a 2010 conference in the European Parliament on Financing culture in the digital era (Cronin 20ro), Maja Bogotaj, leader of the Slovenian Creative Commons project, concluded her talk by stating that there were 350 million CC-licensed digital works on the Web. She went on to note that, although this number may seem large, it indicates that voluntary sharing alone remains confined, except for photography and blogs, to levels that are not suffi- 
cient for the creation of wide cultural commons. It would be ironic, and terribly unjust, if the pioneers who recognized the value of user rights from the start went unrewarded for the success of their works on the Internet. But if they are to be rewarded, we cannot think of this reward as a compensation for lost income: it must be viewed as the recognition of a social benefit in its own right.

\subsection{Social rights for all}

A radically different approach would abandon the quest to create an optimal pattern of incentives, and would instead strive, through the distribution of government rewards, to give creators what they deserve. This may have been what British reformer Robert MacFie had in mind when he urged giving inventors, as an honorarium, what is fair, considering utility, cost of preliminary trials, originality, probability of others making the same discovery, etc. This criterion seems unpromising for a different reason. Natural rights theorists, from John Locke to Robert Nozick, have struggled with little success for centuries to determine the proportion between a person's efforts and the reward he or she reaps. It seems implausible that, in designing a reward system to handle the new technological environment, we could succeed where they have failed.

William Fisher, Promises to keep, p. 208

Are we hoping to succeed where John Locke and Robert Nozick failed? Fortunately, we do not need to achieve such prowess. Measuring the absolute value of one's contribution to culture, or of the effort one has put into it, would be a tall order indeed. ${ }^{17}$ However, a self-consistent measure of the merit of a given work can be based on an estimate of its relative contribution (compared to that of other works) to a particular aspect of our society - in the present case, the non-market cultural commons accessible to all. To recognize this merit financially, we also need to agree on how much we - as a society - are ready to invest in the continued existence and growth of these cultural commons. There are many ways to estimate the relative value of a given work, and our approach (outlined in chapter Io) draws heavily on earlier proposals such as Fisher's, while adding a model of the necessary degree of precision. The second requirement is clearly the greater challenge: can we agree on how much we want to invest - by this particular means of a contribution by all users - into the existence of cultural commons that are accessible and sharable by all?

Of course, it is almost impossible to assign a value to this kind of thing intrinsically. And yet, in practice, societies have to make this kind of decision every day on the value of some "invaluable" sphere of rights, activity or benefits. For example, they decide which resources they will allocate to public health, who will bear 
the costs and what mechanisms will be used to manage these contributions. The same is true for education, justice, the fight against poverty or excessive inequality. It extends to the preservation of the environment or other physical commons. The processes that lead to these decisions are complex, messy, contingent, constrained by inertia and by many other decisions. Our role as analysts is to propose some more solid foundations for these processes.

We suggest starting from one overarching principle and several distinct opportunities and constraints. The overarching principle is to define two positive social rights: the right to access the cultural commons of non-market sharing, and the right to be rewarded for one's contribution to their enrichment. These rights belong to each of us, they do not separate us into two categories, one of creators and the other of consumers. We call them social because they belong to all members of society, but also because each of us must be empowered with the ability to influence how they are implemented.

How much are people willing to pay for these two rights to be implemented (together) in practice? If we are discussing a flat rate paid by all Internet subscribers, we must also consider the impact it may have on other common goods or useful activities. This includes, of course, an impact on future revenues of creators and industry, but also opportunity costs (what people can no longer buy because they paid the contribution). What additional value will it create beyond the cultural commons, such as the sphere of knowledge or capabilities of each individual? We are unlikely to be able to put a figure on this, but it is at least as important as the stability of the profits of a handful of firms that employ very few people in comparison, which has been the dominant criterion in a large part of the debate so far. Chapter 7 tries to sketch out a policy framework in which we can seek, via democratic processes and stakeholder negotiations, to reach a decision on the question of how much.

Copyright law constraints are part of this policy framework, but they cannot, and should not, be its only or even its main foundation. A social rights perspective will enable us to reflect about what is the just relationship between the success of a work in the shared cultural commons and the reward it should get. If copyright royalties grow more than proportionally to sales for physical cultural goods such as books and CDs, as is often the case, who says that a social reward can't grow less than proportionally to usage in the information sphere?

One of the challenges that will face us is the distribution of the collected contribution into shares going to various media and various forms of contribution to works. There are some tentative ground truths that may help here, for instance the time budgets for various media-related activities (production as well as access and usage). But there is clearly room also for collective preferences to express themselves. It does not seem optimal to favor time-consuming media when timeconsumption is often driven not by the value that people assign to a given 
medium but by the associated business models. There also, an approach based on social rights might open up a better decision space.

One of the key benefits of a social rights framework is that it will enable us to revisit the implementation of copyright law with a different set of tools.

\section{Back to copyright}

\section{First sale doctrine and home copying laws}

Historically, two forms of user prerogatives have been defined for copyrighted material. The first one concerns a sphere of activity completely removed from the original right holder: the first sale doctrine instigated in 1908 in the $\mathrm{US}^{18}$ and its European equivalent ("exhaustion of rights"). ${ }^{19}$ The second form of user prerogatives is known as fair use in the US, fair dealing in the UK and most of the Commonwealth, and exceptions and limitations in Europe and internationally. ${ }^{20}$ It provides for limited rights to accomplish certain acts without authorization and, in some cases, without compensation of the right holder.

The first sale doctrine states that a good embodying a copyrighted work can be used by its first buyer and any person to which it is later transmitted as they see fit (including commercial exchanges such as reselling, and, until 1984 in the US, renting). This doctrine was clearly designed for works on physical carriers such as books or records, at a time when the work - more precisely, the information representing the work - could not easily be separated from the carrier (not without a loss of quality, or without a significant effort). As we already noted in chapter 3 , the information revolution has since upset the efficient balance that had been achieved by the combination of copyright law, the first sale doctrine and, to some extent, anti-trust law. New user capabilities (such as making many copies of a work and transmitting them to other persons over a network, without losing the original) give existing recognized user prerogatives a much wider scope. This may be considered legitimate and accepted, or deemed unacceptable, implying a severe restriction of user prerogatives in the digital sphere compared to that of physical carriers. Law-makers on both sides of the Atlantic have struggled with this dilemma for some years. The US Copyright Act of 1976 extended the scope of the first sale doctrine by granting the established user prerogatives to any owner of a lawfully made copy or phonorecord, and not just to the original buyer. In the I980s, various laws, in both the US ${ }^{21}$ and Europe ${ }^{22}$ sought a new balance by restricting user rights, but only to some extent, defining a "private sphere" in which the right to make and exchange copies existed, but now needed to be compensated by the payment of a levy, most commonly on the blank carriers used to store these copies. Seen retrospectively, these laws seem benign in terms of destruction of user rights, compared to what has followed since. In some countries, such as Spain, the "private copy" law was written in a manner that can be interpreted to make non-commercial file sharing legal. ${ }^{23}$ In other European countries, the scope 
of authorized copies and exchanges is much more restricted, and, in most cases, the redistribution of the collected levies is considered extremely unfair. ${ }^{24}$ Still, there is a precedent for salvaging or recognizing user rights by collecting funds on the scale of an entire society, a fact which was seen by some as inspiring. In fact, however, the "private copy" laws represent the first step in a key trend: to regulate the digital world primarily for the benefit of holders of property-like restrictive rights, with some protection for providers of digital technology but no true consideration of the social rights of every individual.

\section{Conditions for exceptions and limitations}

Concerning the conditions under which new exceptions and limitations to the right of reproduction can be created, the Bern Convention, the treaty that regulates copyright internationally, states in its article 9.2:

It shall be a matter for legislation in the countries of the Union to permit the reproduction of such works in certain special cases, provided that such reproduction does not conflict with a normal exploitation of the work and does not unreasonably prejudice the legitimate interests of the author.

These conditions are known as the three-step test, the first step being that the exception applies only in special cases, the second that it does not conflict with the normal exploitation of the work, and the third that it does not unreasonably prejudice the legitimate interests of the author. These conditions apply explicitly to all exclusive rights since the TRIPS agreement of $1994,{ }^{25}$ and were incorporated verbatim in European and national legislation in some countries. TRIPS strengthened and globalized the application of copyright and patents, and critics denounced its provisions as extreme. ${ }^{26}$ However, the three-step test, if interpreted reasonably, as defended by many renowned legal scholars in a 2008 declaration (Collective 2008), would not prevent the recognition of new usage rights. The above quotation includes many qualifications that widen the range of conditions under which the criteria can be deemed fulfilled: "normal", "unreasonably", "legitimate". In addition, it recognizes the sovereignty of legislators in individual countries to create usage rights, provided they respect certain rules. One can also doubt that some existing forms of media publishing and distribution clearly constitute "normal exploitation". Is a form of exploitation in any way "normal" if it survives only by limiting the basic capabilities of human beings in a digital world (copying files, for instance), by organizing universal surveillance of personal communications, by criminalizing tools that are also used for legal purposes, and by threatening individuals with criminal sanctions for conducting activities that did not aim at personal profit and did not demonstrably hurt anyone? 


\section{The creation of a hostile environment}

With the development of digital technology, the spread of the Internet and various related networked applications, we now have close to two billion people equipped with the means - and in most cases with the capability - to produce or exchange copies of works as efficiently as the publishing industry. Obviously, this can only be great news for historians of cultural technology (language, writing, printing, recorded media) or software developers (someone who encodes knowledge and know-how in information technology), but when will we recognize its potential benefit for humanity as a whole? How long until we define a legal framework that regulates cultural industries by incorporating the right to share (without profit motive) as a boundary condition? Over the last 25 years, the law has evolved in precisely the opposite direction.

The intellectual rights policy tragedy described by James Boyle (Boyle 2003, Boyle 2008), Lawrence Lessig (Lessig I999, Lessig 200I, Lessig 2004), Yochai Benkler (Benkler 2006) and our book Cause commune (Aigrain 2005) has given rise to a situation where it seems extremely difficult to modify copyright law in any fashion tending to recognize new usage rights. The effect of the recent evolution of copyright law, in the real world, is to concentrate power not in the hands of authors and artists, but in those of the stock owners of copyright. Interest groups composed of assignees (producers or publishers) and heirs of deceased artists have since endeavored to entrench this change through key cases that establish some jurisprudence precedents and tend to modify the standard interpretation of important words in their favor. The case law is in reality complex and contradictory, but if one follows the most extreme interpretations of these interest groups, "normal exploitation" covers whatever business model a copyright holder chooses. To them, the word "author" means the producer to whom an author (or other creator) has transferred rights ${ }^{27}$ or the grandchild of a deceased author. Consequently, these persons become the ones whose interests are "legitimate".

These extreme interpretations have had an excessive influence on the formulation of the European Copyright Directive of $200 \mathrm{I},{ }^{28}$ such that the latter lists exhaustively, and hence limits, the possible exceptions and limitation to the rights of reproduction, communication and making available to the public. As a result, present European legislation severely constrains any adaptation to the new needs of society: one is simply not allowed to define new exceptions and limitations to the exclusive rights covered by the directive. Many are of the opinion that preempting the future evolution of law in this manner is incompatible with democratic policy-making, and the European Commission itself has shown in its 2008 Green Paper on Copyright in the Knowledge Economy ${ }^{29}$ that it was aware of a possible need for new exceptions and limitations.

Unfortunately, the recent evolution has made the situation even worse: the Anti-Counterfeiting Trade Agreement and some legislative initiatives regarding the enforcement of copyright (Quadrature 20II) are trying to create an additional 
irreversibility in the way exclusive rights are enforced. In summary, for a quarter of a century during which user rights have been progressively cornered, and individual contributors to creation treated increasingly poorly, lawyers who support copyright reform have been faced with an extremely difficult challenge. They are bound to work within the framework of existing law and jurisprudence, and change must be engineered through means available within this realm.

\section{Reform is possible}

The US approach to the definition of alternative reward systems tries to do just this, and it has led to remarkably elaborate proposals. Looking back at the last 6 or 7 years, one is nonetheless forced to admit that we are still very far from putting them into practice. These alternative proposals, together with the consumer rejection of existing offers, played a role in forcing rights holders into accepting to make more works available for commercial download and streaming.

Taking a purely consumption-oriented view on the cultural usage of the Internet, one might think that file sharing arose because of a market failure that is now corrected, and that it is thus no longer needed. This would be a major mistake. For one thing, the battle for a more diverse access to cultural works is by no means over. More importantly, those who reduce culture to the consumption or passive reception of works misunderstand what is at stake today. The dominance of the publishing and distribution industries in the representations of culture in the last century is misleading. Even in this cultural industry era, artistic practice, the social appropriation of works, the sharing of knowledge, the expression of citizens in relation to works have always been essential components of our cultural world. But now there is more to this, and ever more to come. The period when culture appeared dominated by a direct production-to-reception flow will soon be remembered as a mere interlude, between the time when creative and expression works were essentially a matter of craftsmanship, and a new era in which they have become a societal activity. The cultural industry will not disappear, it will find new paths to serve the public.

Most proponents of the legalization of file sharing adhere to the vision we just outlined, whereby sharing is one of the first steps on a ladder of cultural empowerment. However, although brilliant American scholars have made the best cases for stopping the war on sharing, the only (significant) concrete step in that direction in the US was a "proof balloon" proposal from the music industry. Jim Griffin, then a strategist at Warner Music, offered universities a flat-rate license to make music available with file sharing rights, for a fee of $\$ 5$ per month and per student (Orlowski 2008). The other musical majors attacked the idea, while Warner distanced itself from the proposal. To implement it, Jim Griffin then founded the company Choruss but failed to secure access to the needed catalogs.

In Europe and in emerging countries, the situation is different. In several countries law proposals implementing a flat-rate based legalization of non-market file 
sharing were tabled, and/or political parties support the idea. This is the case in France, Belgium, Germany and Brazil, for instance. In other countries such as Sweden and Italy, there are efforts towards using voluntary collective rights management approaches and extended collective licenses to obtain a de facto legalization without changes to the law. Extended collective licenses are ones managed by collecting societies, whose application is extended by law to all authors or rights holders, even if they are not members of a collecting society. Such a scheme can be used to legalize file sharing only if the relevant collecting societies volunteer to apply the scheme, as was the case in Sweden with STIM, and if a law extends it to all authors by default. The latter is unlikely to happen when major content interests oppose the scheme.

All these proposals use a compensatory copyright-based reward system. The debate of the past few years has shown not only that such approaches struggle with the limitative list of exceptions and limitations allowed in the 2001/29/CE Directive on Copyright and Neighboring Rights in the Information Society, but that they are subject to an escalation of compensation requests. This is analogous to the way in which the "Regulatory takings" doctrine was used by powerful interests to oppose environmental protection in the Ig6os and I970s in the US, by claiming it would deprive them of their potential future business. ${ }^{30}$

The war on file sharing continues to escalate, but one reality will not go away: file sharing will not disappear, because many people consider it - rightly in our opinion - as a basic capability in a modern society. ${ }^{3 \mathrm{I}}$ Instead, it is turning clandestine, increasingly using cryptography or content slicing (cutting contents in many small pieces distributed on various computers) to elude detection and prosecution. Innovative minds, which could be working to deliver improved creativity, are wasted thinking up new and clever tools to prevent people from doing things, or even cleverer workarounds to those tools. A dual digital society develops, where law-abiding consumers are passive, while creative prosumers lose respect for laws that they view as serving specific private interests.

Our proposal is an effort to get out of this vicious circle. It is based on tabling the principles for a new social pact:

I. The non-market sharing of digitally published works between individuals is a legitimate right of all.

2. All who have access to this particular form of cultural commons have a duty to contribute adequately to its existence through a flat-rate Creative Contribution.

3. The amount and distribution of this contribution must respect a number of principles regarding: the revenues of individual creators, the financing of the production of new works, the equitable character of the distribution in relation to activities conducted over the Internet, and the impact on the welfare of all. 
The practical implementation of these principles is discussed in chapters 6 to Io. Nothing in our proposed social pact precludes any particular form of legal basis for its implementation. As we will see, when principles 2 and 3 are considered jointly, they ensure compensation in the sense of copyright law, at least if the latter is interpreted reasonably.

An important point to stress here is that we will be in a much better position to find a legal basis that is politically viable, if we first assemble a critical mass of citizens, experts, and practitioners of creativity in support of the social pact. In France, the Création-Public-Internet coalition ${ }^{32}$ has made significant progress towards this goal. This coalition brings together associations and unions of musicians, key film-making figures (producers, directors and actors), the main consumer organization, and Internet user groups. Similar moves are afoot in Brazil, ${ }^{33}$ to a certain extent in Germany, and at an international level in the framework of the Paris Accord ${ }^{34}$ meetings organized by the Transatlantic Consumer Dialog (TACD) and in the Free Culture Forum ${ }^{35}$ of Barcelona. It is better to construct or change the law to achieve valuable and endorsed goals than to allow it to become a cobweb in which one is entangled by years of failure to address the major civilization change brought to us by information technology. 



\section{Defining rights and obligations}

Implementing a new form of mutualized financing of creative activities and recognizing the non-market sharing of digital works is not something one can do at the drop of a hat. An in-depth debate between stakeholders, experts, policymakers and the public is needed to work through the details. For this debate to be possible, a structured proposal consistent with the new approach presented in the previous chapter must be on the table. We now proceed to detail its key components: to which works will the right to share apply and when? Which types of users will be concerned? What will be the rights and obligations of users? How should the amount of the contribution be determined? Some issues that regard institutional and technical implementation (the devils in the details) will be left for discussion in the next part of this book.

Our proposal addresses both the reward of creators for the usage of existing works, and the provision of funds towards the production of new works, or the maintenance of a suitable environment for works to be created and disseminated in. Some authors have proposed financing of artists and projects only, dropping the reward aspect entirely. This model was proposed as early as 2002 at the Blur Workshop on Power at Play in Digital Art and Culture organized at the Banff Center for the Arts, and subsequently elaborated by James Love (Love 2002), now director of Knowledge Ecology International. ${ }^{\mathrm{I}}$ Users have to pay a monthly contribution, which is allocated using a competitive intermediaries model, where various organizations compete to attract funds from Internet users, on the basis of the redistribution policies they publicize. The Blur/Banff proposal was subsequently elaborated to make it more secure against rigging by persons who would agree to cross-allocate the collected sums to each other (Toner 2008). Francis Muguet ${ }^{2}$ (Muguet 2008) designed a "global sponsorship" scheme where a flat-rate contribution by Internet subscribers would be allocated on the sole basis of individual preferences, users deciding (for instance on a monthly basis) to allocate parts of their contribution to various artists and artistic projects. To answer critics who pointed out that it is difficult for individuals to make such decisions at the level of individual artists and projects, Muguet and other promoters of global sponsorship added a provision enabling people to delegate this decision, which would be similar to the "competitive intermediaries" model.

We consider competitive intermediaries to be a key instrument to give individuals direct power over the allocation of funds towards the production of new works, or to organizations that contribute to a better environment for creative 
activity, the dissemination of works or the recognition of their quality. However, it would be a serious mistake, in our opinion, to renounce rewarding the usage of existing works, or to use explicit decisions of allocation for that purpose. Usage of works is more serendipitous and more diverse than the memory we have of it and than our conscious preferences. It would be a cultural loss to abandon expost usage-based rewards.

Internet usage in the non-market sphere is an extraordinary tool for the discovery of new works, precisely because it is not hindered by monetary transactions or decisions on how to allocate funds. If someone is asked to list all the music titles they listened to the last month, or even all the movies they saw or the texts they read, they will typically recall only a very limited proportion of them. For Internetbased media, where works are even more numerous (short videos, photographs, blogs), our memory is even more challenged. This means that explicit decisions or phone polls are not suitable for rewarding the usage of works. Despite this, a live experiment is still being conducted in France today, where phone polls are one of the sources of information for the distribution of the funds levied on blank carriers. This distribution key is as concentrated on a few works (probably those that are heavily promoted) as radio broadcasting. ${ }^{3}$ One of the challenges for the implementation of our proposal will be to find ways of recording the real-life diversity of access to and usage of works. We describe a possible way to address this challenge in chapter Io.

For the time being, let us just note that we plan to use the product of a flat-rate contribution both for rewarding the usage of existing works, and as one of the means to enable the production of new works in a satisfactory environment for creative and cultural practice.

\subsection{Which works to include}

Our first task is to decide on the range of works falling under the remit of the proposed device, i.e. which will benefit from the re-distribution of its proceeds, and officially become freely sharable in the non-market sphere. This choice pertains to:

- which types of media are included;

- whether including a particular work in a particular media is mandatory or optional;

- under which conditions an individual work falling in the above categories is considered to have been published and hence is included.

\section{Media inclusion}

As we already mentioned, many proponents of flat-rate-based legalization of file sharing designed their proposals for musical and moving image contents only. 
William Fisher (Fisher 2004) estimated that the device could later be expanded, and possibly adjusted, to apply to electronic books and games.

On 20 December 2005, the French parliament took everyone by surprise by voting an amendment to the Loi sur le droit d'auteur et les droits voisins dans la société de l'information $\left(\mathrm{DADVSI}^{4}\right)$, which was then under discussion. The amendment created a flat-rate-based blanket license (licence globale) for non-commercial file sharing. It addressed moving image and musical contents. The cinema interest groups and their spokespersons in various political parties immediately clamored that this would be the death of the French cinematographic industry. After a few months of a polemic which was more vaudeville than constructive debate, a new vote was held, which overturned the original. For many, the idea that blanket licensing was ill-adapted to the needs of film, video and TV production became an accepted fact. It is worth noting that a number of movie and audiovisual personalities and stakeholders have now changed their mind on the subject. ${ }^{5}$ When the next repressive copyright law ${ }^{6}$ was debated in 2009, the French Socialist Party tabled an amendment based on the "creative contribution", which would have legalized file sharing, but only for music. Does it make sense to do it only for one medium?

It does, to some extent, but not really. There is nothing fundamental to prevent us from applying the principles stated above to a particular medium only. Convergence may blur the boundaries between media and trigger the creation of mixedmedia forms, but the economy of production of works in various media remains pretty specific. The production of video games is very different from that of books, for instance. However, only a very small part of the benefits of our proposal would be reaped if it was applied to one medium only. Information technology and the Internet are not broken in media segments; there are devices specialized for a given medium (to a certain degree), such as music players, but they work in conjunction with universal computers and are not at all the only devices used to access "their" medium. Innovation in one medium derives inspiration and re-uses ideas from other media. From the point of view of "intellectual property" rights (IPR) enforcement, the Internet is even less "separable" into separate media. Surveillance squads and fake injection providers may do business with the rights holders for a given medium, or use detection algorithms that are medium-specific. However, it is the IPR industry as a whole that pushes for three-strike laws ${ }^{7}$ and for compulsory filtering of the Internet to prevent access to sites that play a role in file sharing. Their attacks on the mere conduit safe harbor of intermediaries, and their efforts to negotiate criminal sanctions for IPR infringements in international trade agreements without democratic involvement of parliaments are the joint work of the entire industry, and often encompass domains well beyond media and culture.

A one-medium recognition of non-market sharing is unlikely to stop the war on sharing and its cortege of harmful effects. Fundamental rights such as freedom of 
expression and communication or the right to privacy would remain at risk and the maturation of ethics, good practice and supporting technology for cultural exchange would still be delayed. In (Aigrain 2008), we analyzed the conditions under which a recognition of sharing and associated financing could be applied to some media only and still produce sufficiently positive results. We listed the following conditions:

- Rights holders for media which are not included must recognize that the infrastructure of the Internet and digital tools are a common good. They should not be allowed to skew this infrastructure to fit the needs of the proprietary model which they wish to preserve, which relies on the scarcity of copies. They would be free to continue to use access control, usage restriction tools or watermarking for their own works, but only provided they do not hinder the use of any other works. None of these tools should become compulsory over the entire information infrastructure just to serve the interests of those who chose to opt out of the Creative Contribution.

- Similarly, they should agree that copyright cannot be enforced at the expense of other, fundamental rights, and that only a judicial procedure can establish that an infringement occurred and lead to the corresponding sanctions. Prosecution can only occur after infringement has been established.

If it was straightforward to obtain agreement to these conditions, this book would be unnecessary. Rather than dealing with the consequences of some media being excluded, it is probably easier to convince a wide range of creative players in each medium to endorse a scheme such as our Creative Contribution. Our proposal is designed to facilitate endorsement by various media communities. The definition of the digital cultural commons it incorporates is crafted so as to ensure a better synergy between digital commons and commercial offers inside and outside the digital sphere. Specific media may raise specific issues, as we will see in the next chapter when discussing the case of books, but all deserve the benefit of a rich cultural commons.

\section{Mandatory or optional character of the inclusion of individual works}

Can each author or creator choose whether to allow his or her works to be shared? This is the case at present: creators are already free to authorize the sharing of their digital works, using Creative Commons licenses for instance. For some types of contents, such as photographs, blogs (including sound and video blogs) and scientific publications, this scheme has been endorsed widely. But there is an important difference: currently, authors and artists who already choose to grant the right to share do not get any direct benefit from having contributed to the cultural commons. One of the key provisions of our proposal is to give them the benefit of a reward for their contribution, should they desire it. ${ }^{8}$ 
Many authors will welcome the potential reward which the proposed Creative Contribution would enable them to get from activities for which, today, they receive no direct benefit. Some might express legitimate doubts over the amount they would receive and the degree to which other sources of income would be affected. However, it is not from creators that we expect the strongest opposition. Even in countries where copyright is rooted in the right of the author, it is the producers, collecting societies and distributors who actually have power over how these rights will be used. Here is a typical example: the French DADVSI law of 2006 endowed the author with the exclusive right to authorize or forbid the application of DRM to his or her works. The only practical effect of this provision was to make the authorization of DRM a standard clause in every media publishing contract: any author who wants a contract has to sign it. Almost every collecting society in Europe forbids its members to use Creative Commons licenses (even the By-NC-ND version, which forbids commercial uses and derivative works). In other words, if they want to grant the right to non-commercial sharing, creators currently have to renounce all commercial usage revenues, including those which fall under statutory licenses such as radio broadcasting of music. Consequently, some US companies such as Magnatune now run a successful business by enabling European musicians to circumvent this abusive power at least partially. Thus, we prefer to allow the entire class of creators to endorse the Creative Contribution or not, after public debate, rather than promoting a situation where each individual must make a decision, given that the latter cannot be shielded from the unequal balance of power between them and publishers or collecting societies.

There is another, arguably much more compelling argument to reject work by work or author by author options: an optional system would lead to complete legal uncertainty for users, with huge transaction costs. The Creative Contribution would no longer represent a foundation for an enlarged cultural commons, it would become just another way to manage exclusive rights, making an already cluttered legal landscape even more complex.

Of course, a proposal for mandatory inclusion would have to be consistent with copyright law. If - as is likely - it is considered an exception or limitation, it will have to pass the three-step test. As discussed above, this is difficult only if one adopts a fundamentalist approach to exclusive rights. It is worth noting that the schemes that contribute most author rights and copyright revenues today would also be considered illegal under such an approach. We will cover the third step of the test ("does not unreasonably prejudice the legitimate interests of the author") when discussing the rate of the contribution. Again, to be consistent, this third step should also apply to the positions of the majors and many collecting societies. When they oppose the Creative Contribution and other similar mechanisms, they are the ones who are unreasonably prejudicing the legitimate interests of the authors they are supposed to serve and represent. 
When does a work enter the non-market sharing sphere?

In principle, the recognition of non-market sharing of digital works encompasses all works that have been the object of digital distribution to the public, whatever its nature (free or paying). However, this principle must be qualified:

- The mechanism must not hinder the author's liberty to determine when they make a work available to the public for the first time: it is automatically applied only once the work is made public, not before. Similarly, the exchange of digital copies of a work in private correspondence does not constitute distribution to the public in the above sense: the recipient of a digital copy of a nonpublished work received as private correspondence cannot share it with others under the Creative Contribution.

- Only a form of distribution which actually makes a work reach the public in digital form counts. For example, showing a film in theaters would not authorize its cam-cording and later exchange, no more than playing a piece of music during a concert would allow the audience to record it for later sharing, or the paper publication of a book its scanning and transmission to others. This saves an essential element of media chronology: the possibility to schedule the public performance, analogic distribution and digital distribution at different times. For movies this sequencing between forms of dissemination of a work currently plays a significant role in the creative economy, in particular where it is defined by law, such as in France. For books, such sequencing might facilitate the transition to an era of digital books. Of course, authors and artists would remain free to authorize these acts if they wish to do so, but this would be outside the scope of the Creative Contribution.

The circulation of works which are not public in the sense of the above will remain subject to the existing implementation of copyright law. It will be possible to sue producers and distributors of copies of these works for copyright infringement. However, rights owners will not be allowed to impose the implementation of surveillance and control tools in the overall information infrastructure for this purpose.

\subsection{Rights and obligations of users and intermediaries}

\section{Scope of rights}

Aside from the limitation to non-market exchanges, we have not so far defined the rights that the mechanism we propose would give to Internet users, nor the associated obligations. In terms of rights, one can envisage two approaches: 
- restrict them to reproduction and communication to the public: each and everyone can receive works, send them and make them available to others (and of course read, view and listen to them, etc...)

- also include remix rights to produce modified works based on the works included in the mechanism.

The first option tends to restrict the activities of Internet users to the access, interactive perception, recommendation and redistribution of works. It has the preference of those who fear that authorizing reuse in non-market sharing would face challenges in countries where moral rights are considered important. One can also argue that the key obstacles to the recognition of the right to share arise from the wish of media companies to maintain or install a monopoly on the reproduction and distribution of digital works. Once this obstacle is removed, the diverse feelings of authors regarding modifications of their works will not block the exploration of new paths.

However, legal constraints have been proven to be far from incompatible with authorizing remixes: in practice, many legal provisions authorize transformations (such as the insertion of advertising in movies) whose effect on the integrity of works is far from negligible. Even countries with a strong moral right tradition such as France are submitted to the Directive on Satellite Broadcasting and Cable ${ }^{9}$ which de facto transmits the exercise of all rights to collecting societies and distributors. William Fisher's initial proposals for a flat-rate-based legalization of sharing (Fisher 2004) included remix rights, but commentators pointed that it would subject the proposal to criticism in moral rights countries, so he later proposed to let authors choose whether to authorize derivative works and remixes or not. Creators would be able to lift restrictions on derivative works through use of adequate licenses - for instance those Creative Commons licenses authorizing modifications, ${ }^{10}$ possibly with conditions. They could also condition such acts to explicit authorization or even payment. This would create a competition between more liberal and more restrictive models, but minimal rights to share would always be granted.

Authorizing remixes is motivated by their key role in non-market collaboration, enabling the creation of new works (annotated versions, reuse in new creative works, etc.). Of course, the remix rights would be associated with an obligation to indicate sources and properly signal modifications and their authors. This second option would avoid some problems that might arise if non-commercial remix rights are not granted. For instance, some might claim that advertising inserts are an integral part of a work, and that it is forbidden to remove them in sharing. One clearly needs to reject such claims, whatever business model protection reasons may underlie them. Some people tear off ad pages from magazines before reading them or giving them to others, and it should remain possible to do likewise in the digital world. 
There is an impact of allowing remixes on reward systems: a flaw in William Fisher initial proposal was that it planned for the reward system to track changes in the form of relative shares of reward to be given to various contributors. This was clearly a source of damaging complexity for the reward system. A simpler approach would consider a remix as a new work, eligible for its own rewards. This means that the remix would have to meet a standard of originality in order to prevent the opportunistic relabeling of works as being a remix in order to reap undue rewards. This is a source of another form of complexity, this time not for the reward system but for verifications and possible oppositions or litigations.

We consider that the choice between the two options cannot be made without an additional debate and exploration of its modes of implementation. Finally, media criticism should not be blocked on the basis of the non-authorization of modifications. The recognition of a right to share is by no means the end of the debate on the scope of user rights, and fair use rights or exceptions are no less needed in an environment where sharing is recognized.

\section{User obligations}

If the Creative Contribution is put in place, users exerting the right to non-market sharing will have only two obligations, besides making sure of course that their usage is not aiming at profit:

- They must respect the right to attribution of works to their authors, as expressed in the Bern Convention. This right has a validity that goes well beyond copyright in the narrow sense of control over copies. In practice, the experience of voluntary sharing under Creative Commons licenses or other free licenses has shown that when the right to share is granted, the attribution of works to their authors is at least as well respected as in proprietary management. The Web culture of metadata, linking and referencing favors the respect of attribution. Even in non-authorized sharing, the $\mathrm{ID}_{3}$ metadata associated to mp3 files, for instance, is much more readily available than the industry-defined identifiers such as those in CD record publishing.

- Users must also abstain from removing or tampering with the unique identifiers included in files representing works, which are used for the collection of data needed to reward creators. The principal motivation for such removal or tampering would be to replace one identifier by another in order to obtain a financial reward by fraud. Cryptographic integrity checks enable the prevention and efficient prosecution of such fraud, but need specific adaptation to the case where derivative works are authorized. ${ }^{\text {II }}$ 


\section{Intermediaries and commercial services}

When an activity is legitimate and legal, providing means to do it must also be legal, whether it is done free-of-charge or against payment. As any infrastructural commons, the commons of non-market sharing will need services that maintain them, ensure they are functioning efficiently, help users to filter or locate contents of interest, ensure long-term conservation, collect statistics about sharing beyond those that are needed to reward creators, or protect the privacy of users. Some of these services will be provided by community efforts at no cost. Others might take the form of paying services. The definition of these useful, lawful services assisting non-market sharing is not a trivial task, however. If the idea is to recognize non-market sharing, commercial ventures should not be able to disguise themselves as providers of means to non-market sharing between individuals, when in reality they would do commercial usage of the works themselves, or of the attention time of users during access to works on a site. This is best illustrated by examples:

- Any service that does not give access directly to works, but only points users to where and how they can obtain them from other individuals, should be recognized as legal, whether it is free-of-charge and advertising-free, or not. For example, the original implementations of Napster or trackers such as the Pirate Bay would both be legal, provided they respect user privacy, of course.

- Services which use advertising or subscription to give access to contents directly, whether for download or streaming, are clearly excluded from the benefit of the right to non-market sharing. They would need to negotiate commercial licenses for the corresponding reproduction, communication and making available (in Europe) rights. Whether one decides to submit these rights to a collective or statutory licensing is another decision. ${ }^{12}$

Between these 2 cases, some limit situations deserve to be studied and debated in detail and are beyond the scope of this book. What about a blogger who posts an article giving access to a video or musical recording and receives some advertising income using technology such as AdSense?

\section{Libraries}

Another important question is that of libraries. Bruno Blasselle (Blasselle 2008) recounts that, in the late Middle Ages, the birth of universities led to an increase in the demand of copies of texts, bound as codices. This led to the development of copy craftsmanship outside monasteries, with specialized craftsmen: copiers, binders, illuminators. With this multiplication of copies went a multiplication of errors, then unavoidable in texts that were often copies of copies. To prevent such errors, repositories were created, where a faithful reference copy was stored: the exemplar. However, the exemplar was not bound as a codex: it remained divided in 
folios or pieces. What for? At the time, the aim was not to prevent copies from being made, but instead to encourage them. However, copying was a slow process. To speed up the production of copies, separate folios could be lent to different copiers: while one copied the first folio, the others could copy other parts of a text. ${ }^{13}$ These repositories of exemplars are the forerunner of an important role for the libraries of the future.

In future, will book and media libraries give access to everyone on the Internet to reference copies of all published works, including those still covered by copyright and commercially distributed? It will certainly be the case one day: part of the role of legal deposit libraries or reference libraries will be to "seed" a reference copy, in the sense of the BitTorrent protocol or - most probably - one of its successors, that is to initiate the chain by which milliards of people can, if they so desire, have access to these works. Openly accessible archives and repositories play this role today in scientific publishing.

However, this will not necessarily happen immediately for domains where public or not-for-profit funding plays a more limited role in investment for the creation of new works. One can imagine a transition period where the role of libraries will be focused on the public domain in the widest sense as suggested by Robert Darnton (Darnton 20II). Giving access - under completely free reuse terms - to digitized public domain ${ }^{\mathrm{I} 4}$ documents is already an immense task, essential to the rooting of our culture in its heritage. Giving access to orphan works and out-ofprint works, which represent the vast majority of works covered by copyright for books and a significant share for other media, is also an essential rescue task. There is no sense in charging fees for access to orphan works, so the latter should become usable under collective licensing without compensation by users, but a guarantee state fund is needed to protect users against the possible claims of rights holders who re-appear after a work has been made available. To enable the republication of out-of-print works, the best approach is to make sure that, after a short moratorium period, the associated rights are transferred back from the publishers to the authors. Many publishing contracts have such clauses, but they must become a legal obligation.

If the Creative Contribution grants a right to non-market sharing between individuals, an open debate will be needed on the status of non-profit organizations, and in particular educative and memory institutions. It would be paradoxical if the recognition of a new non-market sphere restricted the role of existing nonmarket organizations. If the Creative Contribution is limited to sharing between individuals, it must be complemented by a strong fair use doctrine, or by exceptions for research, education, libraries and archives. 


\section{How much?}

In this chapter, we embark on our most challenging task yet: to come up with a proposal for the amount of the Creative Contribution and the manner in which it will be distributed, as a starting point for future debate. We do this in a way that has not really been explored before: we consider the potential of on-line sharing to contribute to the cultural and creative capabilities of each and everyone, and estimate the needs for rewards and support for the production of new works from that perspective.

The "how much" question is easily tackled from a compensatory standpoint: evaluate the losses resulting from the existence of file sharing, then see whether the resulting figure is acceptable for those who will be asked to pay. This simplicity is somewhat deceptive. As we have seen, evaluating the losses to be compensated is far from trivial. First, which alleged losses deserve to be taken into consideration? Should we count present losses, or extrapolate into the future? Are we to compensate creators only, or also investors? Are losses compensatable only if they are fatal, in the sense that any exploitation of a creation would be jeopardized, or should we also compensate those who suffer losses because they stick to a particular mode of exploitation, but might not otherwise? $?^{\mathrm{I}}$ Once the perimeter of losses is defined, we must of course discuss their amount. Terry Fisher did this calculation in (Fisher 2004), estimating the losses to correspond to $\$ 5.36$ per month and per Internet-subscribing household for music and film. But if one were to repeat the calculation today, given the experience of the last six years and the many studies published in the interval, one would find a significantly reduced figure for the compensation of phonorecord-, film- and video-related losses. Even agreeing on a new figure would not be the end of it. There are many other forms of media, some already widespread in the digital sphere, such as photography, and some only starting to invest it, such as books. Furthermore, as we have already remarked, there is no reference upon which to base a compensatory reward on digitally native media: they would be collateral victims of such an approach. In other words, we would neither reward nor finance those who build the heart of the digital cultural commons.

We thus propose to define a self-standing system of rewards and upstream financing for the digital cultural commons, but we have to quantify it on a different basis. We need to answer two questions simultaneously: what is the minimum amount of finance needed to enable the cultural digital commons to develop to its full potential, and what are the limits to what households can contribute 
to this development by means of a flat-rate contribution? How much is enough, how much is too much?

As a preliminary, we must first justify more explicitly why we propose the Creative Contribution to be used to finance creative activity and its environment, and not just to reward the contributors to existing works.

\subsection{Rewarding the present and financing the future}

There is some symbolic beauty in the idea of balancing rewards for existing works with the support for the creation of new works. It is also motivated by a rational analysis: incentives based on ex-post rewards have very different properties from financing of projects, activities or environments. Both have qualities and drawbacks. Ex-post rewards account for the - sometimes unpredictable - attention of the public. From time to time, some perfectly unknown individuals or groups suddenly grab the attention of a large public, vibrate with its feelings and resonate with its thoughts, and thus embody the spirit of a time. Such moments are beautiful, and we were lucky to live through several of them in less than two generations. Each of us is was shaped by the ones they lived through: from the beat generation to Indian music, from the nouvelle vague to hip hop, and from new Asian cinema to cyberpunk novels. There are a thousand such moments, because each of us travels only in a few, though some are visited by so many that they seem universal. When the fluidity of cultural encounters leads to the recognition of a work or author, it is only fair that a reward system acknowledges it. Even in classical cultural circuits, the discovery of new deserving works and creators is not infrequent. All too often, however, ex-post reward incentives based on copyright do not serve the perpetual regeneration of culture, but instead perpetuate existing or predictable successes.

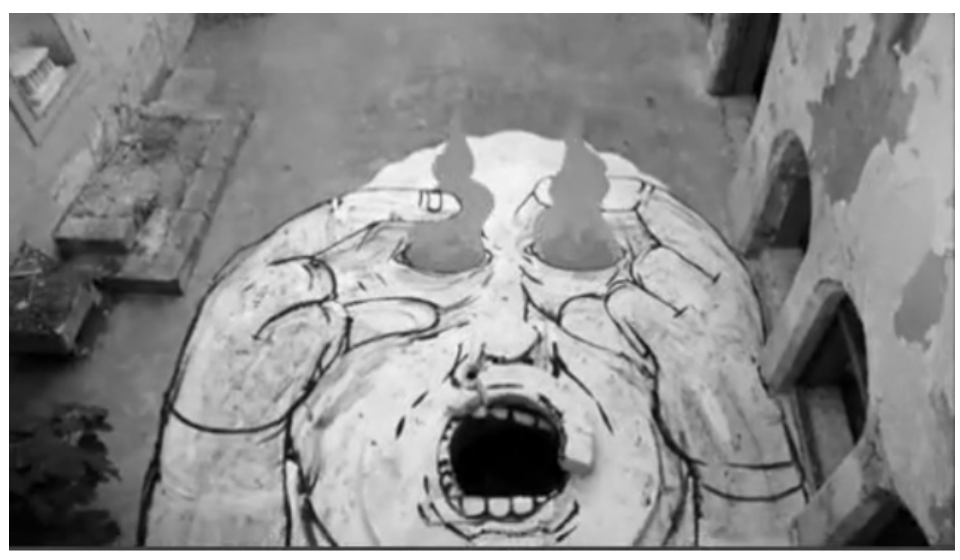

Fig. 7.1. An image from COMBO, a collaborative animation by Blu and David Ellis, http:||vimeo.com/6555161, license CC-By-NC-ND. 
In contrast, a reward system for Internet usage can work on different scales, acknowledging emerging stars as well as creative people with more limited audiences. The French website La boite verte provides a wonderful introduction to the nature of encounters one makes in Internet culture. ${ }^{2}$ This blog does not produce creative works itself: it only points to the works of others. Its author explains what La boite verte is about in the following terms: "It helps me to store cool stuff that I don't want to lose", ${ }^{3}$ and thanks to its existence, we don't lose the cool stuff either. At the time of writing, the site points to around a thousand contemporary and older works. Most of them are remarkable works of art, feats of ingenuity or pedagogy, or simply great fun. The production of many of them has required considerable time and effort. All the sources are referenced with care on La boite verte. Some, such as the animated wall-painting collaboratively produced by Blu and David Ellis illustrated in figure 7.I, have been viewed on the Internet more than 500,000 times. ${ }^{4}$ Others will reach only a few hundred or thousand users. A reward system that specifically takes the Internet into account will reward many more creators ${ }^{5}$ than copyright royalties, as we will see below.

When we look back at the emergence of art that seems truly new, we find what was already there: the Woody Guthrie behind Bob Dylan. We also find things that are less often recorded in bios: environments, places, groups where a know-how, a thought, a voice, gestures were conceived, tried out and appreciated, where styles emerged and matured. The environments of today's Internet culture start with personal computers and home studios (millions of them in Europe or in the US). Next up might be cafes, bars or student halls with open wireless Internet. When we follow the trajectory of creative people, we find other places: bookstore back-rooms hosting creative writing workshops, pubs with open jam sessions, small music production units that are at the same time rehearsal studios and micro concert halls, dance studios, philosophical cafes, squats and hacker spaces in former industrial buildings where multimedia installations and performances are prepared, and some public spaces that are beyond the reach of inspectors who collect fees for public performances. Then there are more institutionalized environments, which subsist mostly on public, community or private funding: art and culture centers, exhibit spaces.

Finally, there is production, the most deceptive word of our debate. When we discuss our proposals for the legalization of file sharing with creators, only very few inquire about lost income. Most have another, more pressing concern: can I do my next project? Can I secure the round-table for my documentary movie? Where do I find the money for production of my next set or album? ${ }^{6}$ Who will finance my photo-reporting trip? How do I sustain a decent editorial team for my collaborative on-line news media? Who will believe in me even when my first projects fail, as John Hammond believed in Bob Dylan? Who will stand in my recording sessions and tell me what's right and wrong? Who will promote me, but in the sense of peer appraisal, like Johnny Cash writing the liner notes for the 
Nashville Skyline album? The term "production" should not be restricted to what Joseph Stiglitz had in mind when he wrote: "Producers whose profession consisted in bringing music to consumers have no raison d'être today. It's like trying to save the horse and cab-man industry in the car era." It points also to what he suggested thereafter: "However, their work can evolve towards a more editorial function, guiding consumers towards this or this type of music."

Many of the environments mentioned above survive without the kind of financing mechanism we advocate. Some dearly need it, but funding each one individually would lead to far too much micromanagement. It is preferable to fund intermediaries who, in turn, will support these decentralized environments. Additionally, a wide range of production and dissemination environments can be direct beneficiaries of a mutualized financing scheme.

\subsection{Rewards}

In this section, we propose a method for designing a reward system for sharing on the Internet, and we apply this method to obtain a detailed proposal. Most of the discussion, however, is not tied to the specific Creative Contribution mechanism which we advocate in this book: the analysis is relevant to any financing model for digital cultural and creative activities. For example, it does not depend on whether or not the model is based on a compulsory contribution. It is worth mentioning that, in parallel with our work, Roberto di Cosmo (DiCosmo 20II) conducted an analysis that covers many similar issues, in particular regarding the use of credits and reward functions.

\section{What is a work?}

Since our rewards are not based on copyright law, we are free to define the work (as a unit that can be rewarded) as best fits the nature of on-line exchanges and the functionality of the reward system. To some degree, we can even leave this decision to the creators themselves. Individual movies or episodes of TV series are better considered as units: allowing a different practice for them would run the risk of biasing rewards. On the other hand, for the purpose of the reward system, it is better to consider a blog or an on-line photographic gallery as a single work, rather than treating each entry or photograph as the unit. For musical recordings, the choice of what constitutes the proper unit could be left to the creators. Creative Commons music websites such as Magnatune, Jamendo or Pragmazic have demonstrated that the notion of an album (a set of musical tracks) remains fully valid on the Internet. ${ }^{8}$ Musicians could decide whether they want usage to be recorded and rewards granted on the scale of an album or of individual tracks. ${ }^{9}$ This choice of albums will decrease transaction costs and management overheads in the system, and make it easier for lower popularity works to reach the threshold leading to a reward. We come back to these implementa- 
tion details when discussing the minimal registration and identifier requirements for works in section Io.2.

The input to a reward system: usage credits for individuals

To design any reward system, one must first estimate how many people are to be rewarded, and at what level. From this, one can easily deduce the total amount of funds that will be necessary. Here we are discussing rewards associated with the level of usage of works. By now, the reader will be familiar with ranked popularity distributions. Such a distribution is the natural starting point to design a reward system for usage on the Internet. To construct the ranked popularity distribution, the compound usage of the works of each creator is recorded, ${ }^{\mathrm{IO}}$ and individuals are listed in order of decreasing usage. Usage may mean different things and can be measured in various ways, which we describe in chapter 10.

There are many unknowns regarding this ranked list. How many people will be on it? What will be the average level of measured usage? Will the measured usage be concentrated on a few creators, or spread over many? All this will be easy to compute or model once we have observed it for a few years. But at this stage, we need to make a few simple a priori hypotheses in order to estimate the overall scale of the necessary funds. To do this, we model ranked popularity distributions using Zipf's law, as introduced in chapter 3 . This type of model provides us with a tool to discuss various hypotheses.

\section{Design choices for a reward system: how to go from usage credits to rewards}

A reward system must transform the ranked distribution of usage per contributor into another distribution, of financial rewards. The conversion from usage "credits" to a reward amount for each contributor is not a straightforward, mechanical process. For a given total cost for the system, it depends on three essential policy choices:

I. What is the minimum number of people we want to be rewarded at or above some meaningful level?

2. Which reward function is used to translate use credits into rewards?

3. What is the minimum amount of reward worth redistributing to a contributor per year?

To answer the first question, we must clarify the main purpose of the rewards. The key promise of information technology and the Internet is to enable a manyto-all cultural society, a world in which very many people contribute works or productions that are accessible to all, many of these works deserving the interest of some, and a significant number the interest of many. Therefore, the primary design criterion for the contribution we are proposing is that it should enable 
many more people to get better at whatever creative or expressive activity they wish to use the Internet for. Getting better may mean learning a skill that is not specific to the Internet: playing an instrument, writing compelling texts, taking photographs or shooting videos. All these techniques become accessible in a different manner thanks to information technology and the Internet. People now have access to tools and practices that were formally reserved for professionals, but to benefit from this new access, one must first learn to use these tools proficiently, discover what one really wants to do with them, experiment and get feedback from others. This takes time, effort and guidance. Helping a sufficient number of individuals in this respect will be our essential guide in making the first design choice above.

By reward function, we mean the way in which a relative level of use is translated into relative rewards. If a given work is 3 times more popular than another, should the corresponding reward be 3 times larger? Less? More? Many people assume that rewards must be proportional to use. This is definitely not the case in the existing copyright systems, where the royalties grow more than proportionally to the sales. A best selling author will receive 50\%, or even 100\% more royalties for each book sold than most authors. This trend is even more pronounced for music. On the other hand, as we will see, in the digital world there are excellent arguments to justify a less-than-proportional reward function. However, there are also constraints on how far we can go in that direction.

We may gain some insight into the appropriate choice of reward function by studying the observed distribution of usage credits. However, we should bear in mind that the present distribution is a complex mix, where traditional attention patterns from the pre-Internet cultural industry rub elbows with emerging manyto-all patterns characteristic of the digital sphere. When the Creative Contribution is put in place, these patterns will blend together, and we must attempt to forecast the resulting distribution to understand how the reward system will work in the long term. This is not an easy task, but models based on Zipf's law allow us to consider a range of possibilities. Figure 7.2 compares the cumulative distribution of attention that could be expected under various scenarios in a fictitious medium-size country where two million people contribute works that are shared on the Internet. The top curve corresponds to a pure concentrated attention pattern, similar to what is observed in large-scale media publishing and present-day copyright royalties. The bottom curve corresponds to a diverse attention pattern, as might be observed if the giant communities of non-market Internet sharing came to dominate access on the Internet. The middle curves show intermediate situations, such as one might expect to see for the first few years after the Creative Contribution is put in place. See appendix B for details. The most concentrated case corresponds to a Zipf law parameter of I.I, and the most diverse to o.8. The intermediate cases have Zipf law parameters of I.o and 0.9, respectively. 


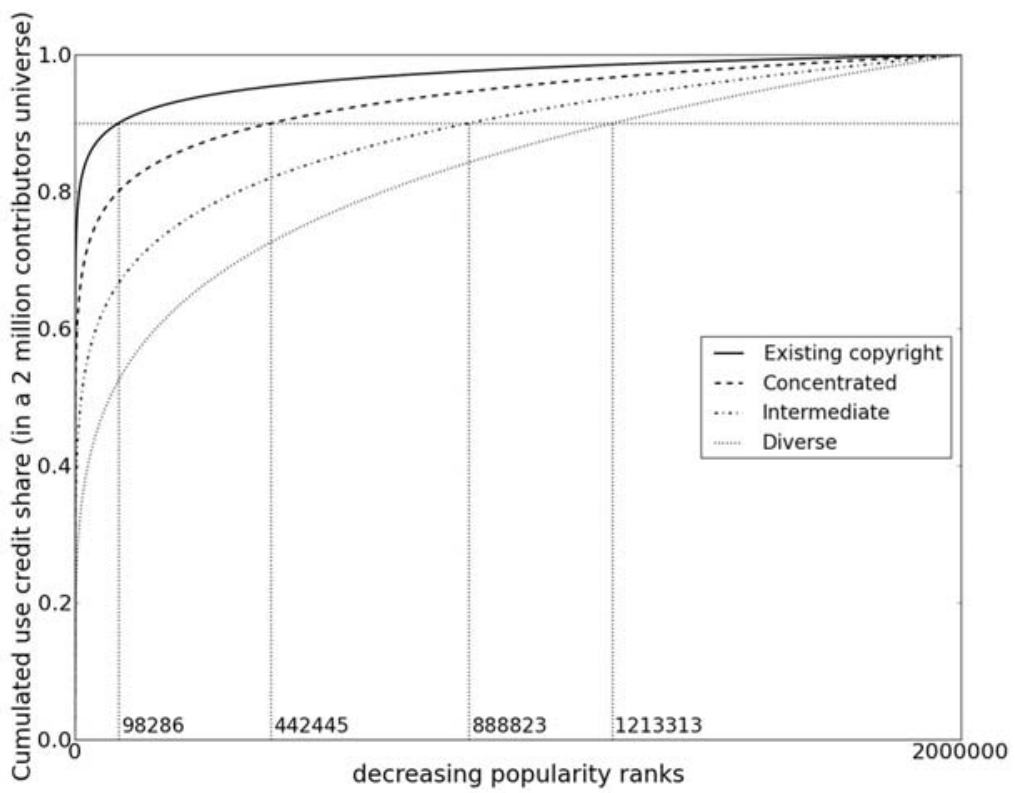

Fig. 7.2. Various distributions of attention to creators can lead to great differences in the number of creators who share $90 \%$ of the usage credits.

It is interesting to look at a horizontal line corresponding to 0.9 on the y-axis. In the first (maximum concentration) case, fewer than I00,000 contributors $(5 \%$ of the total number) receive $90 \%$ of the total attention. This number is very similar to the number of people who receive copyright royalties today in a country of this type. ${ }^{\text {II }}$ The concentration is strongest near the top: fewer than Io,ooo contributors receive $80 \%$ of the total attention. ${ }^{\mathrm{I} 2}$ For our present purposes, the most interesting cases are the two intermediate curves, where the number of contributors sharing $90 \%$ of the total attention is much larger: over $20 \%$ and $40 \%$ of the total creative population respectively. One can expect the actual usage pattern to approximate the higher of the two (corresponding to a relatively concentrated distribution of attention) in the initial phases after the introduction of the Creative Contribution. This is not only because Internet publishing will take time to reach its full potential, but also because established right holders will be likely to register themselves and their works in the Creative Contribution system more rapidly and efficiently. The pattern of usage would then progressively become more diverse, tending towards the third curve, or even the fourth (bottom). These are tentative hypotheses, but whatever the details, it is clear that the distribution of usage credits for individuals will be much more diverse than any of the present distributions for commercial channels, at least after a brief initial period. It will 
also be much more diverse than the present distributions of copyright revenues for any media (with the possible exception of books).

Let's now look at motivations to adopt a less-than-proportional reward function. Some of these motivations are of an economic nature. In the industrial production of physical goods, there are economies of scale in production and distribution. These constitute incentives to focus, to a certain degree, on a limited number of products. It is clear that no such constraints exist in the digital nonmarket world. Many digital sales business models nonetheless rely on concentrating the attention of users on a limited number of works. However, the key reasons to adopt less-than-proportional reward functions are not economic, they are social and cultural. It is a desirable property of culture that some works, and those who create them, will attract the attention and appreciation of very many. It is, however, socially damaging if this attention leads to levels of income and wealth utterly disproportionate to the cost of producing the works, or the income needed to maintain a good standard of living. Rishab Ghosh stressed that, in the digital world, the value lies in the existence of a work, and not in each of its copies (Ghosh 1998). This suggests that as long as a work has proven itself to be of interest - by attracting the attention of a certain number of users - we should value it for itself, and not only with respect to the number of people who appreciated it. This is the true meaning of a many-to-all cultural society, and less-thanproportional reward functions contribute to it.

In Appendix B, we show that when the distribution of usage credits more or less follows a Zipf law, a power law reward function gives rise to another Zipf law distribution for rewards. A power law reward function means, for instance, that a work receiving Io times more attention will be rewarded $\mathrm{IO}^{\mathrm{a}}$ (Io to the power of a) times more than another work. Setting $a$ to $\mathrm{I}$ gives a proportional reward. A lessthan-proportional reward is achieved by using $a<\mathrm{I}$. For instance, if $a=\frac{1}{2}$, the reward will be $\mathrm{IO}^{\frac{\mathrm{I}}{2}}=\sqrt{\mathrm{IO}}=3.16$ times more, instead of Io times more. A lessthan-proportional reward function transforms a concentrated distribution of attention into a more diverse distribution of rewards. For example, if the distribution of usage credits corresponds to the "concentrated" curve in figure 7.2, but one uses a power law reward function with $a=0.8$, one obtains a distribution of rewards corresponding to the "diverse" curve in the same figure. Another approach to less-than-proportional reward functions has been implemented in practice in Sweden. The compensation system for loan libraries uses a "topping-off" mechanism: authors are compensated proportionally to the loans of their books up to the annual median wage $(€ \mathrm{I} 6,000)$. Beyond that, they receive only half of the proportional reward. If their total compensation exceeds $€ 19,000$, they receive only 10\% for any further loans. Whatever one thinks of the idea of a compensation for lending books, topping-off mechanisms are an interesting approach, in particular from a social angle. 
There are, however, constraints on how far one should go with less-than-proportional reward functions. The most successful creators, and those whose interests are tied with theirs, would certainly oppose it. As they still see the reward as a compensation for lost income, they would describe it it as an "unfair" reduction in rewards. We discuss this point further in section 7.4, where we show that provided the less-than-proportional character is not excessive, this position is not an insurmountable obstacle. It is nonetheless clear that a strong momentum of support in civil society and cultural contributor groups will be necessary to surmount it. Even in the absence of a political opposition, it would probably not be considered fair, or culturally productive, to adopt a reward function that is too strongly less-than-proportional. For example, under the cubic root reward function advocated by Richard Stallman (Stallman 2009), a work used by one million times more people than another would only be rewarded roo times more.

There are also limits on the minimum reward to be distributed to an individual, for two related reasons: transaction costs, and the social acceptance of the reward system. There are costs associated with the distribution of the reward. These include management costs for the organization(s) administering the rewards, but even more transaction costs for recipients, who have to register themselves and their works. Distributing rewards below a certain limit would lead to an inefficient system, whose management costs would be disproportionate: they would be criticized by both financial contributors and beneficiaries. Beyond transaction costs, associating a monetary aspect to non-market activities - even in such an indirect manner - is only worth doing if it is not "peanuts". Furthermore, the smaller the rewards to be distributed, the more challenging it becomes to measure usage precisely enough to allocate them, and with enough resistance to fraud (see chapter 10). Collecting societies already set such thresholds on distributing the collected rights. They are criticized because of the manner in which the "undistributed" rewards are handled. The corresponding fees are collected (paid by users), but in most cases, they are distributed to the more favored beneficiaries, on a prorata basis following their other benefits. In other words, the least popular creators are made to subsidize the most successful. Such a problem will not exist in the case of the Creative Contribution: the amount of the fee is computed while excluding the rewards that are not distributed. In the following, we propose a threshold for rewards of $\$ 40$ or $€ 30$ per person per year (for the compound use of all works of which that person is a contributor). Note that one could lower this figure by half without raising issues of costs: the questions of relevance and technical feasibility mentioned above come into play first.

We now proceed to develop a working proposal for the rewards, and to address the key question of what their absolute level should be. 


\section{A fixed cost adaptive reward system}

If the aim of the rewards it is to enable investments in the development of individual and group capabilities, one must ensure that a sufficient number of creators receive rewards that are above the threshold of practical usefulness. Whether the reward is to be used directly to buy tools or services (such as courses), or indirectly by freeing time that would otherwise be devoted to earn a living, a meaningful entry figure is probably in the range of $\$ 200$ or $€ 150^{13}$ per year ${ }^{\mathrm{I} 4}$ per artist or contributor. This does not mean that we should not distribute rewards below that level. However, we should design the system such that a sufficiently large number of contributors receive a truly meaningful - even if limited - financial support.

How many people should the system fund? To answer this, we need to know how many people produce and share cultural works on the Internet. In France and in the European Union as a whole, $20 \%$ of the population aged 16 or older declares having produced and published some contents for sharing over the Internet in the past 3 months (Deroin 2010). Typical works are photographs, blogs, poetry or music. Personal publishing on the Internet is rising rapidly (it doubled in the past 3 years in France) and shows no sign of slowing down. According to the Eurostat 2007 pocket book on cultural statistics (Eurostat 2007), 10\% of Europeans (EU-27) declare that they regularly play an instrument, $15 \%$ sing, $3 \%$ act, I $9 \%$ dance, ${ }_{2} \%$ write texts or poems, $27 \%$ make photographs or films, and $16 \%$ practice some other artistic activity. Another study conducted in France (Donnat 2007) concluded that the predominant pattern associating Internet usage and cultural practices was synergistic: more Internet usage is associated with more non ICT-mediated cultural practice. Olivier Donnat, the author, left open the question of whether this was a temporary trend due to the fact that most present Internet users grew up before the Web, or whether it will still observed when digital "natives" dominate the population. In our opinion the synergy arises mainly from the decrease in TV viewing time, which frees time for other activities; from the empowerment permitted by the use of ICT; and from the fact that physical artistic activity is a natural complement to mediated information. The question raised by Olivier Donnat must thus be revisited: do we want this effect to last? Are we ready to give the young (and the less young) the rights and capabilities that will help them invest in cultural practice on the Internet and beyond? In the US, writing one's own blog or personal web pages was a typical daily activity for $3 \%$ of Internet users ${ }^{15}$ in 2008. This figure is not easy to compare to those regarding usage in the past 3 months. However, a detailed comparison of a range of statistics on cultural practice seems to indicate that significant personal content publication on the Internet is slightly lower in the US than in Europe: $15-20 \%$ of US Internet users, that is $\mathrm{II}-\mathrm{I} 4 \%$ of adults, engage in it.

Our starting point is that, even in the "worst" case in terms of diversity, corresponding to the "concentrated" curve in figure 7.2, with proportional rewards, 
our system should reward at least 2 to $2.5 \%$ of contributors to works published on the Internet at a level above $\$ 200$ or $€ 150$ per year. With the latest known statistics, this would represent approximately 840,000 recipients in the US, 315,000 in Germany and 230,000 in a country such as France. More generally, it would represent about 0.4 to $0.5 \%$ of the population aged $\mathrm{I} 6$ or more in a developed country. ${ }^{16}$ These figures may appear low, compared to the overall scale of Internet exchanges. However, not everyone wishes to receive monetary benefits from their on-line production. ${ }^{17}$ Additionally, for the Creative Contribution to be socially acceptable, the popularity threshold for receiving significant payment needs to be high enough to give the public some degree of guarantee that its recipients deserve the benefits they will receive.

\section{Concentrated (low diversity)}

\begin{tabular}{lllll}
\hline Country & $\begin{array}{l}\text { \# recipients above } \\
\$ 200 \text { or } € 150\end{array}$ & $\begin{array}{l}\text { \# recipients above } \\
\$ 40 \text { or } € 30\end{array}$ & $\begin{array}{l}\text { Total yearly } \\
\text { amount in local } \\
\text { currency }\end{array}$ & $\begin{array}{l}\text { Monthly amount } \\
\text { per broadband In- } \\
\text { ternet subscribing } \\
\text { household }\end{array}$ \\
\hline US & 840,000 & 4.2 million & $\$ 2,659$ million & $\$ 2.64$ \\
France & 230,000 & 1.15 million & $€ 501$ million & $€ 2.32$ \\
Germany & 315,000 & 1.58 million & $€ 704$ million & $€ 2.31$ \\
\hline
\end{tabular}

\section{Intermediate diversity}

\begin{tabular}{|c|c|c|c|c|}
\hline Country & $\begin{array}{l}\text { \# above } \$ 200 / \\
€ 150\end{array}$ & $\#$ above $\$ 40 / € 30$ & $\begin{array}{l}\text { Total yearly } \\
\text { amount }\end{array}$ & $\begin{array}{l}\text { Monthly amount } \\
\text { per household }\end{array}$ \\
\hline US & 1.376 million & 8.23 million & $\$ 2,659$ million & $\$ 2.64$ \\
\hline France & 360,000 & 2.14 million & $€ 501$ million & $€ 2.32$ \\
\hline Germany & 497,000 & 2.98 million & $€ 704$ million & $€ 2.31$ \\
\hline
\end{tabular}

\section{Diverse}

\begin{tabular}{lllll}
\hline Country & $\begin{array}{l}\# \text { above \$200/ } \\
€ 150\end{array}$ & $\#$ above \$40/€30 & $\begin{array}{l}\text { Total yearly } \\
\text { amount }\end{array}$ & $\begin{array}{l}\text { Monthly amount } \\
\text { per household }\end{array}$ \\
\hline US & 1.839 million & 13.75 million & $\$ 2,659$ million & $\$ 2.64$ \\
France & 465,000 & 3.49 million & $€ 501$ million & $€ 2.32$ \\
Germany & 651,000 & 4.89 million & $€ 704$ million & $€ 2.31$ \\
\hline
\end{tabular}

Table 7.1. Number of rewarded contributors and total amount of rewards for various hypotheses on observed diversity or applied reward functions 
We now derive estimates for the global cost of a reward system that satisfies the standards set out above. Table 7.I summarizes our estimates of the total amount of the reward part of the Creative Contribution in three countries: the US, France and Germany. ${ }^{18}$ We have designed the model (detailed in appendix B) so that it can adapt gracefully to variations in the diversity of attention or to different reward functions: when the diversity of observed use is greater or when a less-thanproportional power law reward function is used, the number of rewarded creators is higher, whilst the total amount of the rewards remains constant. One may also wish for the total amount to evolve: we will come back to this point in chapter 8 . The 3 cases presented in table 7.I correspond to three levels of observed use diversity for proportional rewards ${ }^{\mathrm{I} 9}$ or to situations where one used a power law reward function to obtain such a distribution for the rewards. In the worst case, the amounts were calculated such that 2 to $2.5 \%$ of Internet contributors receive more than $\$ 200$ or $€ 150 / y e a r$, and Io to $12.5 \%$ more than $\$ 40$ or $€ 3$ o/year. Our working hypothesis is that the diversity of recorded usage will be limited initially, and grow progressively.

Interestingly, the figures obtained in various developed countries are relatively similar. This should ease the handling of international issues connected to the Creative Contribution somewhat (see section 8.2).

\subsection{Financing production and the creative environment}

We have defined a framework for rewarding the creators of works shared on the Internet. This leaves open the question of which works will be produced. This is certainly not a question of quantity: we live in an era of abundance of creative activity, personal and public expression. However, many fear that widespread non-market sharing of digital works could make it more difficult to invest in the production of some types of works, or more difficult to support some editorial functions, which remain necessary so that quality is recognized and promoted within the vast array of available works. In this section, we explore the contribution that Internet users could make to ensure that rich and diverse works of high quality will indeed be produced and recognized.

We proceed as follows: we first give a tentative inventory of the types of works that could benefit from an additional form of - Internet-specific - financing. We sketch out the global economic framework for the production of works in various media, in order to derive a reasonable global estimate of what the financial contribution from Internet users could be. We then discuss those editorial activities which would run the risk of becoming "orphaned" in a world dominated by a mix of concentrated media industry and decentralized Web sharing. 


\section{Media}

So far, and in particular when exploring the global amount of the reward part of the Creative Contribution, we have not distinguished between works in different types of media. Internet user practice and - increasingly - creative activity tend to treat all media as a global set and blur the boundaries between them. However, for certain purposes, there is a need to consider the specifics of each medium. For example, it will become necessary to do so in order to organize the management of rewards effectively, a question which is discussed in chapter 9. But it is even more crucial when considering means of supporting the production of new works, and maintaining a favorable environment for their recognition and dissemination.

Information and communication technology is used in all media, whether to create new works or to digitize analog works and make them more widely accessible. This does not mean, of course, that activities such as making a film, composing music or performing it, taking photographs, creating visual artwork, or writing texts occur only on computers connected to the Internet. In fact, as information-based tools become pervasive, physical practices and presence, gestures, face-to-face interaction become increasingly valuable. Furthermore, a medium (moving image with a soundtrack, music, photographs, books, blogs, etc.) is much more than the carrier that transmits it. Carriers do matter, of course. Digital and argentic photographs are not the same. Lightweight digital video has a completely different feel to a film shot with a $35 \mathrm{~mm}$ camera. Writing a book such as this one on a computer, using text-editing software, or doing so on a typewriter, or even using paper and pen are completely different experiences, which give rise to different books. Nonetheless, activities of a specific nature are involved in the production of - say - an essay, a piece of music, a film, an animated wall painting such as the one shown in figure 7.I, a poem, or a short format video to publish on the Internet. ICT may help bring the cost of producing an individual work down a lot in some cases, but not in others. For example, heavy investment is usually needed before anyone can start to appreciate a complex computer game or a feature film. Similarly, investigative reporting or indepth field research for a documentary are costly and sometimes risky activities. It may take a lifetime to perfect a skill (playing an instrument, using a brush in painting, picking the right word and the right prosody when writing) sufficiently to enable a particular work to be created, even if information technology and the Internet can contribute to the process.

Historically, different forms of media forms do not disappear as new ones arise: they simply accumulate. We still have theater plays, feature films, novels, pieces of music that last from two to twenty minutes, short news articles and longer research ones, despite the emergence of blogs and on-line video. Some commonly used units are anecdotal, linked to a specific context. In Europe, for example, non-fiction video is often produced in multiples of $\mathrm{I}_{3}$ minutes $\left(\mathrm{I}_{3}, 26\right.$, 
52) ... because of advertising time and programming grids in TV channels. Some of these constraints will no doubt disappear in the future, but the notion that an in-depth documentary needs between 45 minutes and two hours will not go away.

Widespread on-line sharing will pose another, quite distinct challenge. Publishers will no longer act as an a priori filter of what is made public. Informative, creative and expressive works will be available in such abundance that it will be impossible to sample them all in order to identify those that please us. How will we find what is worthy of our attention? Of course, publishers will not disappear, and they will continue to spot works or artists which are starting to attract some recognition, and help them reach a new level of quality. This artists and repertoire ${ }^{20}$ activity will become increasingly vital. One can only spot new and emerging talents and help them to mature if an adequate environment for this early recognition exists. In certain contexts, such as collaborative news media, the importance, quality or reliability of information must be assessed in a very short time frame. It takes a long time, and a lot of money, to build and maintain the competence to do that. (Stalder 2006) analyzed the different types of filters that are necessary complements to the abundance of information, public expression and creativity. $\mathrm{He}$ distinguished between filters on contribution that are necessary for free/open source software development, filters on truth or validity that are necessary in science, and filters on interest that are necessary in the access to creative works. One could add filters on relevance and accuracy for collaborative news, filters on originality for blogs, etc.

\section{Expensive resources in a world of abundance}

Can the financial contribution of Internet users be made to address such a heterogeneous set of needs? We will certainly have to be modest, at least initially. Some domains, such as science and more broadly academia, already have their own resources and rules of governance for the publication of new works. They need to be maintained and defended, in times where many would like to cut the resources and circumvent the governance. New mechanisms can address specific needs, such as encouraging individual citizens and society as a whole to play a more important role in defining the aims of scientific research, and to contribute to it. Some digital-native domains have found their own solutions for sharing compatible investment: they use a commons-based peer production model, for instance of free/open source software. Even in these domains, some innovation aspects would benefit from an innovative financing scheme: see (UNU-MERIT 2006). On balance, we have chosen to treat databases or software as beyond the scope of the Creative Contribution (see FAQ 3 in chapter II). The latter will be more efficient and better managed if it focuses on the universe of creativity, information and expression in the public sphere. 
We propose to identify a number of media-specific needs, without any claim to exhaustiveness, but representative enough to allow us to estimate the global needs for support for creation and its environment.

\section{Contribution to media production}

Our concern here is not to compensate the motion picture, video or music industry, or even individual creators, for possible damage they would suffer due to the legalization of non-market file sharing. The question we seek to answer is: how can Internet users make a useful contribution to the existence of works that will be shared on the Internet? Today, this existence depends mostly on decisions made either by private investors (major companies, independent production firms or labels, investment funds using tax credit schemes, news media and television companies) or by one of many government-administered and often peer-managed funds. Individuals who are not themselves involved in production merely manifest their appreciation in the box office, in TV audience figures, in CD, DVD or download sales ... and via file sharing. If information technology and the Internet have the potential to bring about one great transformation, it might well be the greater role of individuals in supporting the production of new works and in making choices about which works will exist and who will produce them.

Resources from individuals are already being pooled to support projects in many domains. Kickstarter, whose motto is "Fund and follow creativity", defines its mission as:

Kickstarter is focused on creative ideas and ambitious endeavors. We're a great way for artists, filmmakers, musicians, designers, writers, illustrators, explorers, curators, promoters, performers, and others to bring their projects, events, and dreams to life.

We know there are a lot of great projects that fall outside of our scope, but Kickstarter is not a place for soliciting donations to causes, charity projects, general business expenses, or raising funds without a specific goal. ${ }^{2 I}$

Kickstarter allows people who have a project to "pledge it" for a given amount. They commit to realize it if and only if this minimum amount is raised. Donors then have a time limit ( $\mathrm{I}$ to 90 days) to pledge money, which will actually change hands only if the required minimum is reached. The amount requested varies from a few hundred dollars to a few tens of thousands. Kickstarter reached general media coverage when the Diaspora free software project for building a decentralized, user-controlled, privacy-aware social network, which was pledged for $\$ 12,000$, collected more than Io times this amount. At that point, its 4 young promoters asked people to stop donating to them and fund other projects instead, as they did not see how they could usefully spend more money. Most projects on Kickstarter are not for building tools, but for creative endeavors in art and media. 
The average individual support to one project is between $\$ 30$ and $\$ 50$. Many projects are for video or complex visual arts production, whose total cost is in the range of tens of thousands of dollars.

Similar intermediaries exist in many countries. Some follow the participative funding intermediary model of Kickstarter (open to all projects), while others are more like "labels" or "co-production firms", pooling resources for the projects of a group of federated artists. Nicolas Dehorter (Dehorter 2010) has compared both models and concluded that the participative label or co-production model in intrinsically fragile. He reached this conclusion mostly on the basis of examples from the music industry. Although one such label, MyMajorCompany, was highly successful early on, this success was based on a single case (the artist Grégoire sold 700,000 albums as a result of a project initially supported by 347 sponsors $^{22}$ ), and many other participative labels such as Spidart have gone bankrupt or struggle for survival. Typical funding needs, even for expensive musical recording production are not more than $\$ 50,000$ to $\$ 100,000$ (see below). Some intermediaries such as Sellaband ${ }^{23}$ and MyMajorCompany offer investment returns to fans who support a project, but there is no compelling evidence that this leads to more funding than disinterested donations.

In film and video production, developing direct participatory production to the necessary scale is a real challenge. The French organization Touscoprod ("everyone can be a film producer") collects funds for feature film projects. They define their standard of success: "A film with 2,00o coprod[ucers] who have contributed a total of $€ 100,000$ is a real measure of success." ${ }^{24}$ This amount is valuable, but is only a minor financing source for a feature film with a typical budget of several million euros. Even for smaller budget ventures such as documentaries, short formats or light-weight productions, it is hard to see how participative production intermediaries could really expand enough to play a decisive role in a many-to-all cultural society, unless resources are pooled on the scale of an entire society. This is exactly what the Creative Contribution can bring: a means for participative production to reach the necessary scale, in a society where users are co-producers. This role should be seen as a complement to participative funding intermediaries such as Kickstarter, and not as a replacement for them. Their role as quick and agile grassroots resource collectors will remain immensely valuable.

The model of competitive intermediaries presented above on page 79 is the ideal vessel for society-wide pooling of production resources. Organizations such as those we have already mentioned would register to be placed on a list of potential recipients, subject to guarantees of transparency, public reporting, and good governance of funds. Each year (to avoid the frequent repetition of a complex choice) Internet users would decide to allocate given percentages of the production part of their financial contribution to intermediaries of their choice. 
The global production context

\begin{tabular}{|c|c|c|c|}
\hline Media & US & Germany & France \\
\hline Motion Picture & $\$ 15,500$ million $^{a}$ & $€ 1,070$ million $^{a}$ & $€ 1,100$ million $^{b}$ \\
\hline TV & $\$ 10,000$ million $^{c}$ & $\begin{array}{l}€ 1, \infty 00 \text { million } \\
\text { approx. }\end{array}$ & $€ 800$ million $^{b}$ \\
\hline $\begin{array}{l}\text { Music/phonorecord publishing } \\
\text { (excl. fabrication) }\end{array}$ & $\$ 3,000$ million $^{d}$ & $\begin{array}{l}€ 700 \text { million (ap- } \\
\text { prox.) })^{f}\end{array}$ & $€_{410}$ million $^{e}$ \\
\hline $\begin{array}{l}\text { Book publishing } \\
\text { (excl. printing and binding) }\end{array}$ & $\$ 2,800$ million $^{d}$ & $\begin{array}{l}€ 700 \text { million (ap- } \\
\text { prox.) })^{e}\end{array}$ & $€ 310$ million $^{e}$ \\
\hline $\begin{array}{l}\text { Global estimate considering other } \\
\text { media }\end{array}$ & $\$ 45,000$ million & $€ 6,000$ million & $€ 4,500$ million \\
\hline
\end{tabular}

Table 7.2. Estimates of yearly production investment for various media in three countries. Several figures including the global estimates are to be considered approximative. Sources: ${ }^{(a)}$ UK Film Council Statistical Yearbook 2010 (2008 data), http:||www.ukfilmcouncil. org.uk/statsyb2010. ${ }^{(\mathrm{b})}$ Statistiques du Centre National de la Cinématographie (2009 data for motion picture, 2007 for TV, http:/|www.cnc.fr|Site/Template/T6B.aspx?SELECTID= $1724 \& i d=121 \& t=3 .{ }^{(c)}$ Estimated by the author from Bureau of Labor Statistics, total payroll for motion picture and video industries, http:||www.bls.gov/oco/cg/cgso38.htm. (d) Estimated by the author from 2010 Statistical abstract, US Census Bureau, tables 1091, 1092 and 1102, and cross-checked against the number of titles published in 2008 and their average cost of production. ${ }^{(e)}$ Estimated from Chiffres Clés 2010, DEPS/Ministère de la Culture, http:||www.culture.gouv.fr|nav/index-stat.html and (Robin 2007): the methodology described in the box on page 106 leads to significantly lower figures for phonogram publishing. ${ }^{(\mathrm{f})}$ Extrapolated from the figure for France, assuming twice as many titles and triple the turnover.

Now comes the hard question: what should be the overall amount of production contribution for various media? A detailed analysis of media needs and possibilities will require stakeholder debates and research that are beyond the scope of this book. We only attempt to make a gross estimate of a reasonable global budget, as a tentative basis for future debates. We start by reviewing, in very general terms, the economics of the production of works in various media: film, video and TV, music, computer games, book publishing, multimedia, visual arts intended for on-line distribution, etc. From these, we obtain an approximate global figure for the yearly investment in the production of new works, and then propose a contribution level for our Internet user-driven financing proposal.

This analysis is used only to set the global amount, and not to determine the actual distribution of funds to recipients: that will be based on user preferences.

Table 7.2 gives gross estimates of the investment in the production of new works for various media in three countries. Some methodological warnings are 
required: we consider here only the investment needed to obtain the first copy of a work, excluding costs associated with the production of copies, distribution, retail or promotion. This is a logical choice, since we are concerned here with the existence of works, rather than their distribution. Regarding the estimates for phonorecord and book publishing investment, where figures are not directly available from reliable public statistics, we used two methods, cross-checked when possible: one based on the proportion of the global turnover invested in the existence of works (typically $15 \%$ ) and the other on the payroll of publishers and their providers (for instance recording studios). Refer to box below for a detailed treatment of the case of production investment for musical recordings.

\section{Example: Estimation methods for the investment in musical recordings}

In order to derive an estimate for the investment in new recorded music works in the US, we assumed that $15 \%$ of the turnover of the sound recording industries reported by the Census Bureau is directly invested in the existence of new works: this gives a figure for 2007 of $\$ 3,000$ million, which is probably an overestimate. We then cross-checked it against the fact that II 5,000 albums were published in 2008, of which only IIo sold more than 250,000 copies, a mere I,500 topped Io,000 sales, and fewer than 6,000 sold more than I,000 copies in official circuits. On should note that these figures confirm an extreme concentration in sales, but also indicate that the small producers sell their albums in non-official circuits. We estimated the cost of production of one work. It was reported to be $\$ 150,000$ for the production of a high-quality album in (Fisher 2004, note 27) quoting (Hull 1998-2004), but only $\$ 125,000$ in (Bourreau-Gensollen 2007) quoting (Vogel 200I). Such albums are extremely likely to reach the threshold of one thousand units sales, even after the recent decrease of CD sales. For other often self-produced - albums, we assume a lower average cost of $\$ 20,000$ : (Bourreau-Gensollen 2007) quote a minimal cost of \$10,000. Most authors signal a gradual decrease in production costs, except for the most notorious artists. This second estimation figure leads to global estimates of production investment ranging from $\$ 2,340$ million to $\$ 2,930$ million. Note that our analysis is independent of the targeted channel (physical record or downloads, for instance through iTunes), as we consider here only production costs for an initial reference copy of the work.

Our estimates remain approximate, but they are sufficient for our macroscopic approach. Some important forms of media are missing, such as newspaper and periodical publishing ${ }^{25}$ or computer and video games. The statistics for some of 
the relevant sectors do not always include the outsourced production, for instance for on-line broadcasting, publishing and photography. An important global indicator is the total revenue of independent artists, writers and performers, which was $\$$ II,900 million in the US in $2007 .{ }^{26}$ Our broad summary estimate for all media represents our best effort at taking all relevant aspects of production investment into account.

The total figures are impressive: $\$ 45,000$ million in the US, $€ 6,000$ million in Germany and $€ 4,500$ million in France. They will remain so. Many sincere media analysts, observing information technology and the Internet from an outsider's perspective, view it as a hostile environment, ineluctably imposed on media by a cruel fate. They warn of a "black hole" that will swallow all of the content-related industry. ${ }^{27}$ Digital photography and the music and moving image industries are daily proof that this view is misconstrued. It would be just as wrong, however, to assume that no additional financing source for the production of quality works will be needed. By focusing the debate on these caricatural, frontally opposed views, we run the risk of creating a situation where concentrated media publishers promotes a few high-budget works, while Internet production proliferates but remains limited in ambition. What we suggest is an intermediate route which is financially tractable but carries strong ambitions: a contribution by Internet users (through the competitive intermediary scheme) at the level of $5 \%$ of our estimate of today's total production budget in the US and $7 \%$ in Germany and France. The higher percentage is a partial compensation for the weaker per capita investment in production in these countries. ${ }^{28}$ It would come to $\$ 2,250$ million in the US, $€ 420$ million in Germany, and €315 million in France.

\section{Orphan editorial activities}

In the information technology and Internet age, editorial activities are transformed, but they remain very important. It is becoming increasingly difficult to find funds for a number of these activities. Most challenging are those which serve many different users, each of whom is ready to pay only a little for this service. Universal information services such as search engines or geo-localization can be financed at a very low per user level, which makes them compatible with funding through advertising. More specialized activities, such as news analysis, investigative reporting, photo-reporting or documentary film-making, music labels (not in the phonographic sense, but as organizations in which a collective style and individual artists mature), or stock archives struggle to find resources. Photography is most helpful to understand the processes at play, as it is the one medium that has fully undergone the digital revolution. Digital photography is a huge and flourishing industry. More valuable photographs are taken and disseminated everyday than ever. Flickr alone, the leading photograph hosting site, hosts more than 4,000 million photographs, including more than 175 million under Creative Commons licenses. ${ }^{29}$ More people live from being a photographer than 
ever: according to the US Bureau of Labor Statistics, 152,000 persons were employed or self-employed as photographers in 2008 , a number which grew and is expected to grow at least as fast as the total number of jobs. ${ }^{30}$ However, photograph stock archives and photo-reporting agencies are in deep crisis, except for the news agencies that supply a limited number of daily photographs to the world's entire media industry. The citizens of the world are now competing with news agencies for visual coverage of almost every kind of event, but they can hardly invest in photo-reporting in a faraway country. For professionals, a flourishing art market for photography compensates only partially for the difficulty in carrying out long-term personal projects. The additional financing scheme for production which we sketched above can do a lot to renew the development of these activities.

Editorial and archival activities are undergoing a fundamental transformation: they are moving downstream, and this is a huge challenge. It is one thing to receive a few tens or hundreds of manuscripts, to listen to a few hundred tracks, to view a few hundred portfolios or exhibits, but how does one recognize and publicize quality in an ocean of works? All content-hosting and voluntary sharing sites are struggling with this challenge. Flickr has its "best of the week" and "photo of the month", Jamendo and Pragmazic their hundred most listened to albums. It is hard to do much better than a basic filter that eliminates the real junk, followed by random sampling and self-amplification (the selected works automatically receive more attention). To counter this, sites also show the most recently accessed contents or a randomly chosen selection, to give them a chance. They try collaborative rating and advice systems. This is great exploratory work, but there is a lot of room for improvement.

Archiving is even more of a problem. In the past, limits on the rate of fabrication of content objects and on access to the means of production made it feasible to aim at archiving everything one could gain access to (which then mean physically have in one's possession). Today, archiving must be selective, and explicit choices must be made, but who has the legitimacy to make them? Public policy and public funding have an immense role to play, but it is also worth trying the societal route, to enable collaborative archiving.

\begin{tabular}{|c|c|c|c|c|c|}
\hline Country & Rewards & Production & $\begin{array}{l}\text { Ex-post editorial } \\
\text { and archival ac- } \\
\text { tivity }\end{array}$ & $\begin{array}{l}\text { Total yearly } \\
\text { amount incl. } \\
\text { management } \\
\text { costs }\end{array}$ & $\begin{array}{l}\text { Monthly } \\
\text { amount per } \\
\text { broadband In- } \\
\text { ternet subscrib- } \\
\text { ing household }\end{array}$ \\
\hline US & $\$ 2,659$ million & $\$ 2,250$ million & $\$ 225$ million & $\$ 5,348$ million & $\$ 5.31$ \\
\hline France & $€ 501$ million & $€ 315$ million & $€ 32$ million & $€ 883$ million & $€ 4.08$ \\
\hline Germany & $€ 704$ million & $€ 420$ million & $€ 42$ million & $€ 1,215$ million & $€ 3.98$ \\
\hline
\end{tabular}

Table 7.3. The total financial needs for the Creative Contribution (management costs are discussed in chapter 10). 
In order to enable further exploration of these challenges, we propose to allocate an additional ro\% of the production share of the Creative Contribution to these domains (ex-post editorial activity and archiving) .

We now have all the elements in hand and are finally ready to summarize the total amount necessary for the Creative Contribution. The results corresponding are shown in table 7.3 (2009 data in most cases).

One issue remains to be addressed: will the home copying law fees be subsumed (absorbed) in the new contribution? As this has consequences in terms of relations to copyright law, we address this issue in the following section.

\subsection{Passing copyright-law tests}

We now have a macroscopic view of a reward and financing system for digitally published works on the Internet. When associated to this system, is the recognition of the right to non-market sharing (as delineated above) acceptable from a copyright-law viewpoint? Some will challenge the question itself, because they consider the right to non-market sharing as a fundamental human right, taking precedence over the temporary, property-like monopolies of copyright, or because they consider that this right is already recognized in their countries, for instance in Spain. Some will object, on the other hand, because they think that no exception should be tolerated to exclusive rights beyond those already granted. To the former, we express our deepest sympathy and understanding, but remark that it would be a shame for the right to share to remain unrecognized in most countries because of a failure to satisfy the requirements of judges and policy-makers. To the latter, we point out that their approach, if applied in the past, would have ruled out the copyright and author rights schemes which contribute the most resources to creative activity today. For example, we would have no radio or TV statutory licensing and no home copying laws. Creators would enjoy a fortress of exclusive rights in which they would quietly starve, unable to conduct creative endeavors, because most creative activity requires the existence of fair use rights, exceptions and limitations. Instead, let us try to view the situation, for a moment, as an open-minded copyright lawyer might.

First we will discuss the main question affecting the compatibility of the Creative Contribution with copyright law: its impact on the economic rights of creators and investors. We will then briefly address two additional aspects: the relation with legislation on the circumvention of technical protection measures, and that with moral rights issues, when relevant.

We analyze the economic rights aspect by considering four questions:

- How will the revenues of authors, performers and other contributors be affected?

- How will be the ability to finance the production of new works be affected? 
- Will investors be unduly deprived of the benefits of their investment without being properly compensated?

- How will the global cultural welfare be affected?

The best available guide to understanding the present impact of file sharing is the recent paper File Sharing and Copyright by Felix Oberholzer-Gee and Koleman Strumpf (Oberholzer-Strumpf 2010). The paper primarily covers the case of music, which most commentators consider to be the sector most adversely affected by file sharing. The key findings in this careful study are that:

- File sharing Internet traffic grew by a factor of ten between 2003 and 2009, the weekly traffic growing from one terabyte to ten terabytes. This growth can be explained partly by the increased presence of video in file sharing, ${ }^{3 \mathrm{I}}$ and to some degree by the intentional pollution of file sharing networks. It leaves no doubt, however, that the war against file sharing has failed to slow it down. ${ }^{32}$ The paper also provides interesting elements on the geography of file sharing, showing in particular that the three countries studied in this book (the US, Germany and France, and the latter two in particular) contribute to global file sharing at a higher level than expected from their share of Internet users.

- The cannibalistic effect of file-sharing on sales of phonorecords, predicted by many observers, is in fact weaker than expected. Oberholzer-Gee and Strumpf note that no serious study ascribes more than $20 \%$ of the recent decline in sales to file sharing, the most rigorous ones finding no evidence of a specific negative impact. In addition, as shown by many other studies, sharing increases the demand for concerts and raises concert prices, a source of revenue which is already much more important for artists than sales of recordings and publishing.

- The authors propose a micro-economic model and use it to demonstrate that file sharing is unlikely to discourage individuals from choosing a musical career or from investing in the production of works.

- Finally, they claim that the music industry overall had an increased turnover in their period of study (ending in 2007 for this aspect). They include in the music industry the sales of devices such as iPods, which is a debatable choice. However, they still focus on a relatively narrow range (sales of phonorecords, concerts and music players). When taking the wider scope we used in earlier chapters, including recording studios, music publishing, musical instruments and teaching, it is absolutely clear that the music economy never stopped growing in the US.

This summarizes the effects of file sharing today, which is mostly clandestine. What will happen if non-market sharing is recognized for an extended set of media, and the new reward and financing mechanisms of the Creative Contribu- 
tion are implemented? Our claim is that the revenues of creators will be significantly improved, that better incentives will exist for the production of new works, and that overall cultural welfare will be significantly improved. As for the media industry, its fate will depend on its own choices. One can only hope that it will eventually abandon the doomed fantasy of maintaining a business model reliant on the scarcity of copies of works. It will then be able to explore the many paths that lie open to it, and to prosper by serving the public's needs. In any case, only those investors who refuse to explore sharing-compatible business models will suffer losses.

We propose to illustrate our claims by analyzing each of our four questions above for one given medium, selected to shed a specific light on the matter. For artist income, we use music; for investment, the motion picture industry; for the sectoral economy, the book publishing sector. We could have chosen other examples: there is plenty of evidence that investment in digital recordings, or income for writers, will fare better with the Creative Contribution, but a detailed treatment of every case is beyond the scope of this book. The analysis of cultural welfare must, of course, be done globally.

\section{Artist income for music}

It is interesting to compare table 6 in (Oberholzer-Strumpf 20I0), which lists the 35 highest revenues of musicians in the US, with figure 7.3, which plots the 300 highest rewards from the Creative Contribution (all media included) in three scenarios ranging from a more concentrated distribution to a more widely spread distribution. ${ }^{33}$

In the first ("concentrated") hypothesis, 84 artists receive more than $\$ 2$ million per year, and 168 more than $\$$ I million. In the second ("intermediate") hypothesis, 49 artists receive more than $\$ 2$ million and 106 artists receive more than \$I million per year. Even in the last ("diverse") hypothesis, I8 artists receive more than $\$ 2$ million and 43 more than \$I million. In comparison, table 6 in (Oberholzer-Strumpf 2010) shows that only I6 artists received more than $\$ \mathrm{I}$ million in 2002 from recording sales. The year 2002 is the last year for which this kind of data is available, and is also the time at which the recording industry's revenues peaked. We must of course take into account the fact that recipients of the Creative Contribution rewards come from all media, and not just music. But even if only one-fifth of the highest recipients were musicians, it is absolutely certain that these best-selling artists will be more than compensated for the possible loss of phonorecord sales revenues arising from a legalization of file sharing. One might even consider that the reward level for the best-selling artists is excessive, and that distributive justice would be better served with a more widely spread or topped-off distribution. When we consider artists and other contributors as a whole (rather than just the best-selling few), it is even more evident that they will benefit from the Creative Contribution. Our study of France (Aigrain 2008) 
showed that less than $20 \%$ of the copyright and neighboring rights royalties actually distributed to musicians come from the private consumption of music goods (including downloads, but excluding home copying fees). In France, this represents $€ 60$ million. Now let us imagine that the legalization of file sharing leads to a $20 \%$ (€I2 million) reduction in this income. The rewards to musicians from the Creative Contribution are expected to compensate this potential loss io times over. $^{34}$

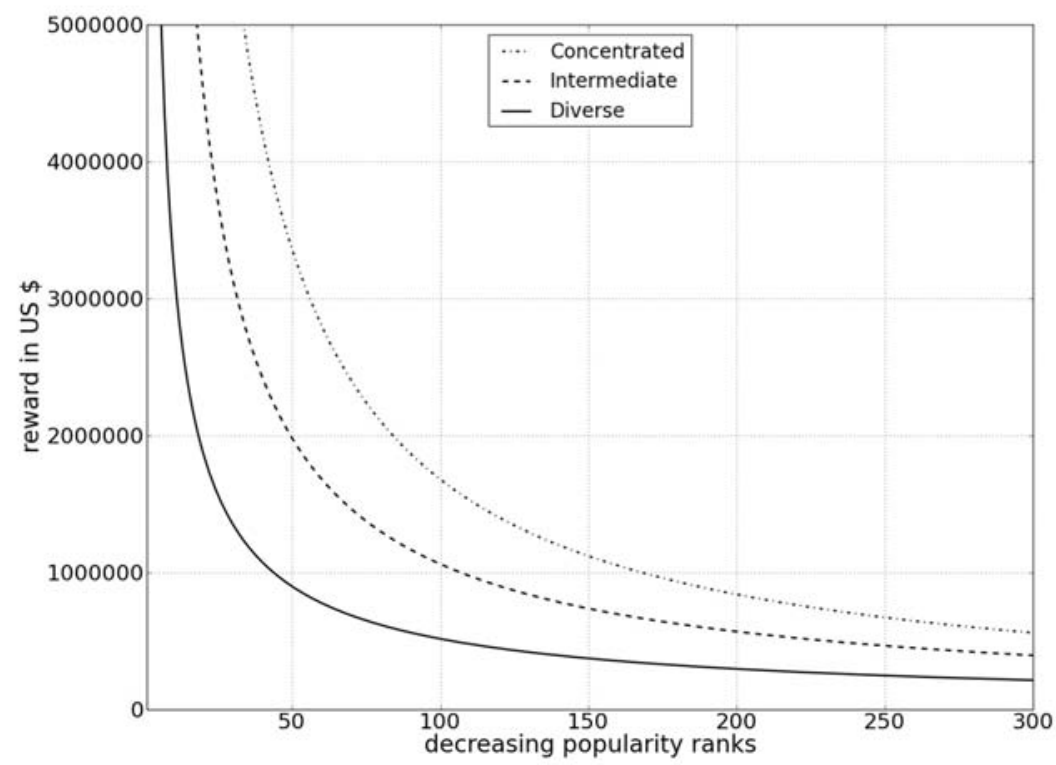

Fig. 7.3. Estimate of the 300 highest rewards from the Creative Contribution in the US, for all media in various hypotheses of distributions.

A new paper by Julie Holland Mortimer et al. (Mortimer et al. 2010) helps us understand better how concert revenues evolved in relation with file sharing. Using data from Pollstar, a company that tracks concert activity in the US, for the period between 1995 and 2004 during which music sharing exploded, ${ }^{35}$ the authors show that the number of concerts and their total revenues increased by $18 \%,{ }^{36}$ that the number of artists participating in concert tours almost doubled in that period, and that, unsurprisingly, the number of concerts per group fell by $23 \%$. This data does not include smaller venues. Overall, it is a great illustration of the relation between sharing and the culture economy: there is nothing to compensate for in terms of harm, and great challenges in terms of the revenues of individual artists due to the new scale of creative activity. 
That the revenues of artists - considered as a class - would significantly increase if a reasonable flat-rate financing by Internet users would be put in place was never really in doubt. The only claims to the contrary were based on a comparison with a totally fictitious scenario, in which a mix of control technology, universal surveillance, repression and brain-washing of consumers would be used to enforce the scarcity of copies of works. In such a scenario, consumers would be forced to pay monopoly prices to access musical recordings. If the industry supporters of this scenario succeeded in imposing it, it is not at all clear that they would actually share much of the profit with artists, who in the meantime would have lost their alternative means of reaching the public.

\section{Film and video production}

As we saw earlier, the motion picture and video industries, considered globally, are in excellent shape: their revenues in the US grew from $\$ 72,000$ million in 2004 to $\$ 82,000$ millions in $2007 .{ }^{37}$ All source of income are doing well, in particular the box office and DVD sales. ${ }^{38}$ Only licensing for television programs are stagnating or slightly decreasing, a natural trend when viewing time decreases, which we can only acknowledge as great news ... provided the freed time is used for valuable purposes. Similar trends are observed in European countries. Despite recurrent complaints of the video publishing interest group in France, ${ }^{39}$ DVD sales are also doing well there: they slightly decreased in turnover for a few years, while still increasing in volume, and recently increased also in turnover thanks to the growing publishing of back catalogs and the entry of Blu-Ray DVDs on the market. ${ }^{40}$ All this occurs while screams of how piracy is devastating the industry are louder than ever. A few years ago, one could think that file sharing had not exerted its effects because of limits on bandwidth. Today, moving image contents are widely shared, through means ranging from newsgroups, BitTorrent $\mathrm{P}_{2} \mathrm{P}$, to direct inter-individual transmission on USB keys. Illegal streaming and download sites also thrive.

Despite this global increase in turnover, there are concerns for the future of production. Let us take a little tour in the world of French motion picture production, which occupies a special place in the French political and cultural imagination. In France more than in other European countries, cinematographic production has been resilient in the face of the inexorable rise of TV and American productions. This was thanks to a variety of public policies, promoted across the political spectrum but particularly on the left. Then, just as new activities started to arise that truly competed with TV's hegemonic control over human time, cinema tied its fate to the latter by making more than half of its production investment directly dependent on TV channels. ${ }^{4 \mathrm{I}}$ This dependency was sealed by the Tasca decree of 2001: ${ }^{42}$ paying and free-to-the-air TV channels are obliged to invest in motion picture production and must diversify their investments to some extent. For a decade, this was the saving grace for the French cinematographic 
production. The present situation is paradoxical, as the initial reason for directing funds from TV into cinematographic production was to compensate for the reduction in the number of theater viewings, whereas these viewings are significantly increasing today (CNC 2010), particularly among the heaviest Internet users ( 15 - to 24-year-olds and white-collar workers), and this despite the development of home cinema and file sharing. Many presently fear that TV channels contribution to film production will inevitably be eroded, because of the diminution of TV viewing time and the growing reluctance of TV channels to program movies. This erosion will not be fast, as there are legal obligations that can only be renegotiated when the license of a channel is renewed, but there will no doubt be demands from TV channels to lower their contribution.

The other half of investment to motion picture production in France comes from very diverse sources: various public subsidies, mostly specific to certain activities (writing, independent production), tax credits for SOFICA (production investment funds) and, increasingly, regional government subsidies. An original mutualized mechanism, the Commission d'avance sur recettes, in which levies on theater tickets finances refundable advances on future revenues for the production of movies, is less important today than it used to be but remains a qualitative reference. Of course, private investment also plays a role, particularly for very highbudget movies produced by the majors. At the other end of the scale, some documentaries owe their existence to smaller production companies and to the investments of the directors themselves. Last but not least, another form of private financing is product placement, which plays an increasingly important - and polluting - role. Pre-financing via DVD sales plays a very minor role in France, unlike in the US.

Media chronology, the scheme by which producers of movies are able to combine sources of income (and the related advance payments) for modes of distribution that are sequenced in time, is undoubtedly challenged. There is a realization that once a movie is distributed to the public in digital form by any means, most of the remaining part of media chronology becomes a fiction. In addition, the major companies have started to realize the interest of flooding all distribution channels simultaneously (except for TV). Does this mean media chronology will disappear? Two important aspects will remain possible to implement: the sequencing of theater projection and digital distribution, and the delayed free-to-air TV broadcast. We have been extremely careful in defining when a work is digitally published in order to separate, when it is desired, theater distribution from later digital distribution. Projection in movie theaters will not be considered making a work public, in the sense that would allow file sharing between individuals. TV broadcast can remained delayed where legal provisions exist to this effect. Contrary to what is often stated, it does make sense for a TV channel to program a movie, even though it has circulated in file sharing. People do not watch movies on television as a primary form of access, they do so because of the convenience 
of set programming: the limit of flow broadcast is also its asset. This double minimal chronology will save the essential sources of financing for movie production in various countries, from the US to France, not to mention India, the biggest movie producer by far.

Despite all this, the current balance of funding for audiovisual production will clearly be rocked, whether or not non-market file sharing is recognized. The most demanding stakeholders (Clubdes 13 2008) highlight that the current organization of funding is unsatisfactory, and that continued major dependency on TV is suicidal for cinema.

In the US, though the 6 major companies (Time Warner, Newscorp, Viacom, Sony, Walt Disney and NBC Universal) account for $80 \%$ of the box office ${ }^{43}$ and have a very strong control over distribution networks, most of the production is outsourced to production companies. ${ }^{44}$

Overall, the matter is not so much to compensate for a negative impact of file sharing that remains to be proven, but rather to contribute a new form of financing, particularly for independent motion picture production and moving image production targeted at the Internet. A probable new source of financing of $€ 70$ to I40 millions in France and \$375 to 750 millions in the US will be extremely useful in this transition period. If a consensus exists on the need for a higher contribution in a given country, it can be implemented without compromising the social and economic balance of our proposal, but one should note that the share of the overall support to production attributed to specific media will result from the preferences of broadband Internet subscribers.

\section{The book publishing economy}

Books deserve a special treatment. More than half of copyright revenues concern written works, simply because copyright was designed for authors of books, partly to protect them from abuse by publishers in the time of printer/publisher privileges. However, part of the book-publishing industry is currently engaged in an interesting form of self-destruction. The manufacturers of eBooks reading devices and some publishers have built a common conviction that they could sell electronic versions of books at monopoly prices, ignoring most of the reduction in production, distribution and commercialization costs, whilst retaining the exclusive control over copies of books that they enjoy in the paper book world. ${ }^{45} \mathrm{It}$ won't be long until the industry wakes up to a situation that is quite different from that which it bargained for. The best-selling eBook readers will be those whose DRM protections are weak. They might sell in large numbers, but this will generate only limited revenue for publishers, and even less for writers, except for a few best-selling authors. If the publishing industry consents to the enclosure of knowledge behind the bars of DRM, when digital technology could be used to make it more accessible and more re-usable, both authors and readers will be resentful. 
(Fisher 2004) judged that it was too early to include books in a flat rate-based authorization for sharing. This was an understandable position at the time. Our position today is the exact opposite: it is urgent to install a playing field where publishers will face the role of sharing in the culture of the digital era. Authors and publishers will remain free not to get involved: paper books will not disappear any time soon, simply because they are wonderful human technology artifacts, the many functions of which it will take generations to outperform. We purposely took care, when delineating the right to share associated with the Creative Contribution, to exclude digitizing of copyrighted analog carriers, such as paper books, from its scope. However, there are fascinating opportunities to explore the synergy between paper books and the non-market sharing of digital books. Many non-fiction works, or even successful fiction works released under Creative Commons licenses, such as Cory Doctorow's Down and Out in the Magic Kingdom (Doctorow 2003) and For the Win (Doctorow 2010), or John Sundman's The Pains (Sundman 2008) have already exploited this synergy with success. Even selling DRM-free digital eBooks is compatible with sharing, if the prices are reasonably set and the suppliers provide added-value services.

Book-publishing production is flourishing today, the number of published titles undergoing a constant and significant growth. The US title output went down from a high of 295,523 in 2004 to 275,232 in $2008,{ }^{46}$ but this should be contrasted against very dynamic growth in the UK: $28 \%$ in 2005 , for instance. ${ }^{47}$ Additionally, self-publishing of print-on-demand books has undergone an explosive growth, overtaking classical publishing in number of titles in 2008, the total US output rising $38 \%$ to 560,626 titles. In France, the number of published titles went up from 70,I44 in 2006 to 76,205 in $2008 .{ }^{48}$ A similar growth is observed for unit sales and turnover (around $3 \%$ per year).

The sales of books, and even the copyright revenues of authors, are still much less concentrated on a limited number of titles and writers than for other media (Robin 2007). Worrying trends are nonetheless visible: an increased concentration in classical and on-line distribution channels, except for direct self-publishing or sales by small publishers; a growing role of promotion - often TV-based for a few books expected to become best-sellers; growing difficulties in financing editorial added-value positions within publishing organizations. In other terms, the book industry is already living in the Internet age: all goes well at the macroeconomic level, there are difficult issues in the market structure, pressure on added-value functions, and publishing lives in fear. It has even started to blame its pains on piracy: SNE, the French Publishers' Union used the results of an interesting study that investigated the unauthorized offer of books for sharing (Daval 2009) to promulgate its vision of an industry under siege, which will reach the safe haven of digital sales only if piracy is eradicated. Curiously, among the Ioo most pirated authors according to the study, a quarter were philosophers, which seems good news for the cultural taste of pirates. The author of the study 
himself warns against any conclusion that digitally shared books should count, even in part, as lost sales, and criticizes previous affirmations to this effect in an earlier study (TeraConsultants 2008).

Copyright law creates no obligation whatsoever to ensure that economic players can successfully implement a particular business model, however much the consumers might reject it. The constraints we have here are of a different nature. The Creative Contribution must pass the three-step test for the creation of new use rights, in particular the second condition, that possibly protects economic players beyond the author: "does not conflict with a normal exploitation of the work". In the US, it must also be immune to challenges under the fifth amendment takings doctrine, which prohibits taking "private property ... for public use, without just compensation". We will not put an end to debates on what may or may not constitute a "normal exploitation of the work". The best we can do is to take our lead from renowned legal scholars from many countries, who called for A Balanced Interpretation of the Three-Step Test (Collective 2008). The declaration includes this point:

4. Limitations and exceptions do not conflict with a normal exploitation of protected subject matter, if they

- are based on important competing considerations or

- have the effect of countering unreasonable restraints on competition, notably on secondary markets, particularly where adequate compensation is ensured, whether or not by contractual means.

As we will see below, adequate compensation is there, but there is also a much more important competing consideration. Not recognizing the non-market sharing of digital texts restricts user rights, compared to paper books, in a manner which undermines the very foundations of culture and knowledge, whereas information technology and the Internet can, and should, enlarge access to culture and knowledge to an unprecedented level. A form of exploitation of works in the digital domain that does not permit an individual to share copies of works that $\mathrm{s}$ /he has acquired legally with other individuals, without aim of profit, is not "normal".

The takings doctrine issue is of a different nature. It is far from certain that we should consider copyright or other intellectual restrictive rights to constitute property, in the sense of the fifth amendment. The introduction to a debate on this matter (Ghosh et al. 2007) stated:

The Federal Circuit recently answered this question with a definitive "no" in Zoltek v. US, holding that patentees can obtain remuneration from the government for unauthorized uses of their inventions only at the legislative prerogative of Congress. Zoltek has tremendous implications for the constitutional 
security of these property rights, establishing far-ranging regulatory power over patents and potentially impacting innovation policy in ways that neither jurists nor patentees have realized just yet.

A takings doctrine challenge brought on behalf of publishers, against the recognition of a right to non-market sharing associated with the Creative Contribution, would face even more obstacles than the patent example cited above, since copyright is even further away from physical property. It should nonetheless be noted that a just compensation defense would also be solid. Even the most criticized studies such as (TeraConsultants 2008), mentioned above, acknowledge that it is only the digital publishing revenues that are possibly harmed, and only to a limited degree. However, the specific investment for digital editions is limited, as evidenced by the fact that, in very few years, new titles arising from personal and small publishing became more numerous than classical publisher titles (Jones 2009).

In subject areas such as computing, where digital books are increasingly predominant, the synergy of digital publishing and sharing, based on providing a complementary added value, is illustrated every day by publishers such as O'Reilly (OReilly 2002). It is hard to predict how this will translate for works of fiction, except for science fiction which has also started going down that route. But in the future, when a publisher commissions or acquires copyright on a book, they will do so with the full knowledge of the possible exploitation forms, and of the need for them to respect the right to share. Most of their catalogs were acquired when the digital exploitation of works was not even imagined nor mentioned in copyright transfer contracts. In a similar vein, DVD publishers who specialize in back catalog films have resisted the rise in video file sharing even better than other segments of video publishing. ${ }^{49}$ It is thus only for recent works, and for those published between now and the time at which the Creative Contribution is implemented, that the issue of a compensation might arise. This issue will actually be the object of a debate between publishers and authors: should the publishers, in when they are copyright assignees, perceive the rewards of the Creative Contribution on behalf of authors? The French photocopying compensation law for books created a levy mechanism whose products go half to publishers and half to authors. In our case, the Creative Contribution will bring its share of new financing to publishing projects, if Internet users are convinced that they need and deserve it. The same law stated that the compensation for photocopying must be paid directly to authors, and publishers are not authorized to keep it, even when the author's account for the book in question is negative (i.e. when the advance they received from the publisher exceeds the royalties on sales). This seems wise. We further discuss this issue in relation with contracts on page 120.

We have to abandon our analysis of the constraints of publishing books or any other form of content there. A number of competition-based measures are 
needed to ensure a sound economic environment for cultural works, and to maximize the benefits of the Creative Contribution. We will outline these complementary measures in the next chapter.

\section{Global cultural welfare}

The word welfare has two relevant meanings in this context: the socio-cultural well-being of citizens, and the overall economic benefits. The socio-cultural benefits of file sharing are so obvious that one might wonder at how little research has been done to quantify them. The access to digital culture provided by a legalized non-market sharing between individuals would constitute an immense benefit, of particular value to the poorest segments of the population, who cannot afford to buy more than a limited number of cultural goods. This point will be of particular relevance when discussing the social acceptability of a flat-rate contribution. This social benefit will be limited by the fact that access to broadband Internet for socially disadvantaged households remains significantly lower than for richer households. ${ }^{\circ}$ It is nonetheless increasing at a fast pace, and could be further improved in the US by the National Broadband Plan..$^{\mathrm{I}}$ (OberholzerStrumpf 2010) provide clear evidence that the US citizens of today are free-riding on the investment made by European and Japanese citizens and governments in broadband, in order to access files shared by Internet users in other countries. A study commissioned by the Dutch Ministries of Economic Affairs, Justice and Education, Culture and Science, and conducted by two independent research centers (TNO 2009), started doing the obvious: evaluating the impact of file sharing (unauthorized or not) on the global welfare. The study concludes that there is an indisputable positive effect on cultural diversity and access to culture, and thus a net positive impact on social welfare.

The long-term evolution of global economic welfare, in the absence of a scheme like the Creative Contribution, is less obvious: consumers enjoy a net positive effect, but in the long term, providers could suffer a decrease in sales of some products, and thus it is uncertain if the net positive effect would last. Implementing the Creative Contribution would put a new burden on households (paying for the contribution) but also bring them increased benefit (legalized file sharing). Meanwhile, producers of contents would receive new benefits, in the form of financial rewards and improved conditions of their activity.

\section{The fate of levies for home copying}

Home copying laws led to the creation in most developed countries of fees on blank carriers possibly used for personal copying of copyrighted sound recordings and video. In Europe these fees are distributed taking taking in account the new exclusive right of "making available" granted to phonorecord and videorecord producers further to the 1996 WIPO treaties. ${ }^{52}$ These fees have created a mixed bag of benefits and drawbacks. Many analysts challenge the principle itself 
of the levies, i.e. that people would have to compensate right holders for legal acts conducted in the private sphere. The levies led to the distribution of benefits to a wider set of artists, in particular music performers. At the same time, their distribution is considered by many as unfair, because the actual distribution has very little to do with the actual use for copying of the carriers. The only key used in this distribution that bears some relation to actual copying is the result of polling by phone interviews on what was actually copied on carriers, an indicator that is known to be biased towards the highly promoted contents (the only ones that come to mind among the interviewees). The fate of the levies seems to be sealed due to another trend: copying of copyrighted works on carriers is much less frequent, and only if one considers any computer storage device as a carrier, as tends to be done in Germany, would there remain a reason to maintain them.

At first sight, it appears a natural choice to subsume (dissolve) the home copying levies within a new contribution associated with the right to share. However, this risks to resulting in importing compensatory approaches within the frame of a reward system which we do not want to be compensatory in nature, and in giving an excessive power to videorecording and phonorecording producers in the negotiation of the new contracts between creators and producers (see below). We thus prefer to let levies on carriers phase out of their own course, even if this means the temporary co-existence of the two systems.

\section{New contracts between creators and producers}

The Creative Contribution rewards a new form of exploitation of works. It cannot be considered as included in previously negotiated contracts between authors, performers, other contributors and producers/investors. Existing contracts will have to be amended, and new contracts made to contain explicit provisions. Two approaches are possible: one which will make the rights to the rewards nontransmissible and another which would accept their being assigned to producers with some form of benefit for authors. We expect that the chosen approach will differ across countries depending on their social and cultural tradition. It would be a mistake in our opinion to try to impose one of the two schemes. What is essential, if the transfer of rights is made possible, is for the conditions under which it would be negotiated to empower creators in the negotiation: the original rights should sit with them and them only. In Europe, this may open a debate on the proper compensation of the inability to enforce the "making available" rights of producers for existing works. A transition scheme could be put in place if necessary. For future works, producers (as a whole) are likely to benefit from the production support side of the Creative Contribution to a higher level than their existing revenues from blank carrier copying levies. 


\section{DRM and moral rights}

Recognizing the right for individuals to share digitally published works without profit motive is one thing. But what if technical protection measures (TPM) make it impossible, and the legal protection of these measures against circumvention precludes removing them $?^{53}$ In some countries, TPM are protected against circumvention, even when this circumvention is necessary to allow a legal use of the contents to which they are applied. This is in particular the case in the US, following the Digital Millennium Copyright Act (see ChillingEffects), and in the transposition in some European member states of the 2001/29/CE Directive on Copyright and Neighbouring Rights in the Information Society. Other EU member states have chosen to subject TPM to the respect of authorized use (Ireland) or to make circumvention legal when it is necessary for a legally recognized use. The 2010 copyright reform law proposal in Brazil contained even stronger provisions with direct sanctions for DRM that hinders legal use $\mathrm{e}^{54}$ (Doctorow 20Iob). In addition, the legal protection of interoperability, enshrined in article 6.2.b of the directive, has been sufficient to protect software such as DeCSS against circumvention claims. DeCSS permits access to content-scrambled DVDs for the sake of playing them in free/open source software systems. In practice, this software is used globally for a variety of purposes, while its distribution remains illegal in the US. Legal frameworks that permit the use of TPM to prevent users from exercising their legal rights will need to be revised in order for the right to non-market sharing to be a reality.

The large content industry has played a strange game regarding TPM/DRM: it periodically recognizes that consumers hate DRM and that no commercial offer constrained by them will be accepted. Nonetheless, it keeps pushing for new DRM systems to be implemented, applied to a variety of contents (in particular video and text), and installed in IT and media devices. It will be a side benefit of our proposal to install a more stable situation in this respect.

An important clarification should be made: access control, for instance in order to give access to works only against payment, remains perfectly $\mathrm{OK}$, and circumventing it is illegal hacking of a site. This is an important enabler for commercial offers that are compatible with file sharing, and essential to sites that provide information whose value is limited to a short time frame, such as news media. One will have, nonetheless, to be careful that this is not abused to reinstall DRM in their usage control sense. This would be the case, for instance, if a provider were to give access to works for payment with a technical protection that requires some authorization each time the content is accessed or copied.

Finally, we must return to a possible opposition to our proposal on the basis of moral rights, in countries such as France and Germany. Moral rights are a complex package. Some of them, such as attribution, are internationally recognized and can be fully applied to the right to share, as they already are in Creative Commons licenses. Similarly, our proposal fully respects the right of divulgation: it 
does not apply prior to the publication of a digital version of a work, authorized by the author (whether commercial or non-commercial). Other moral rights such as integrity have never been effective in the personal sphere: we can cut a magazine to make a collage, or cut a book into slices in order to allow several people to read it in parallel when books are in short supply (just like the handlers of the Exemplar did, in the Middle Ages, to ease its copying55) without risking a moral rights infringement case. The right to integrity is not threatened by the right to share as we have delineated it, certainly not as much as it is by editing of movies for on-flight projection or advertising inserts in TV and satellite broadcasts. Other moral rights such as droit de retrait et de repentir are left over from times when authors, if they changed their mind about a particular topic after publishing something on it, could not use the Internet to publicize the fact widely. ${ }^{56}$ In any case, they have no bearing on the right to share. Moral rights are therefore not a possible source of challenge to the legitimacy of our proposal.

Only a fundamentalist conception of exclusive rights, which would prohibit any regulation of the exploitation of works, could be opposed to the Creative Contribution and the associated right to share. But then, copyright and author rights would become such a straight-jacket that creativity would suffocate in their arms.

\subsection{Is the Creative Contribution socially acceptable?}

The payment of the Creative Contribution will put a financial burden on households. Is this burden legitimate? Are the benefits provided sufficient to justify it? These questions must be answered from two perspectives: first for households as a whole, and then more specifically for low-income households, for which this additional burden may not deliver the intended benefits if it makes access to broadband Internet unaffordable for them.

For the more general assessment, we compare the amount of our flat rate contribution to other relevant components of a typical household budget:

- household cultural expenditure in the wider sense;

- household fixed Internet access expenditure;

- household expenditure for mobile communications.

Each of these reference figures is useful for a different purpose: cultural expenditure because it would be self-defeating for a proposal that aims to encourage a wider participation in culture and creative activity, to compete excessively with existing spending for cultural purposes; Internet access spending because the flat-rate contribution will be collected by Internet Service Providers and, even if it is a separate payment, will risk being perceived as a part of Internet costs; and finally, mobile communication costs because they are an example of a form of household expenditure that has grown immensely in the last fifteen years in 
many countries, including for low-income households, while it could be significantly reduced by adequate competition policy measures.

Our concern is not whether the shousehold cultural expenditure will grow or not in general: the development of a huge sphere of non-market cultural activity is beneficial in any case. We are just trying to check that the payment of the Creative Contribution will not in itself impact cultural consumption excessively.

In France, the average cultural expenditure per household in the strict sense $\mathrm{e}^{57}$ is reported by the Eurostat pocket book on cultural statistics (Eurostat 2007) as €I079 for I999. A study by CREDOC researchers has estimated that the 2007 cultural expenditure in France was 20\% higher (Maresca et al. 20II). We have serious reservations on some figures in this study, and think that the real figure is higher, but we adopted this figure for sake of caution. (Lacroix 2009) gives a much higher figure for 2007, where cultural goods, services and equipment reach $€ 2 I 14$ per household, but this includes equipment such as computers, which are used also for non-cultural purposes. In Germany, the figure for I999 is €I, 374 and we have assumed a $20 \%$ higher figure for 2007 . Unfortunately, more recent figures are difficult to obtain or compare. In the US, the spending per consumer unit for entertainment and reading (that does not include amateur practice) has grown $37 \%$ from $\$ 2,050$ per year in 1999 to $\$ 2,816$ in $2007 .{ }^{8}$ We add $10 \%$ to the US figure to account for amateur practice. (Cammaerts-Meng 20II, see page 7) give figures that confirm an increase of consumer spending for "leisure" in that range in the UK. ${ }^{59}$ We thus obtain the figures listed in Table 7.4 for various countries.

\begin{tabular}{llll} 
& US & Germany & France \\
\hline Cultural expenditure & $\$ 3,098$ & $€ 1,649$ & $€ 1,295$ \\
Proposed total yearly Creative Contribution & $\$ 63.8$ & $€ 47.7$ & $€ 49.0$ \\
representing (percentage of cultural expenditure) & $2 \%$ & $2.9 \%$ & $3.8 \%$ \\
\hline
\end{tabular}

Table 7.4. Yearly household cultural expenditure (2007)

In all cases, the burden imposed on households by the Creative Contribution is very reasonable in comparison to their global cultural expenditure. The risk that the contribution would lead households to limit other forms of access to culture is therefore limited. However, there are noticeable differences in percentage from country to country. These differences are in part explained by the fragility of statistics on cultural expenditure, in part by the fact that a higher share of public spending is devoted to culture in Europe than in the US (which can be seen as a hidden form of cultural spending through taxation), and in part by a generally higher consumption expenditure per household in the US. One should also note that the cultural expenditure of households with a broadband Internet connection is higher than the expenditure of average households. ${ }^{6}$ 
In France, the average cost per equipped household for a fixed line Internet connection is $€ 33$ per month (ARCEP 2008). Some $90 \%$ of the population has access to offers costing $€ 30$ per month at most, against which they receive $8 \mathrm{Mbits} / \mathrm{s}$ download and IMbits/s upload speed or more, integrated free voice over IP telephony to wired lines in most countries, and a TV over IP access to more than a hundred TV channels. Costs for pure broadband Internet are even lower in most regions in Germany, while open integrated triple-play offers are less developed because of a greater role of proprietary TV contents offers and cable TV providers. In the US, the costs of broadband Internet access are similar, but for an inferior quality: in 2010 the Federal Communications Commission has upgraded its requirements for the definition of broadband to $4 \mathrm{Mbits} / \mathrm{s}$ download and I Mbits/s upload (FCC 2010), a figure that was well below any fixed line broadband market offer that year in Germany or France. If the commitment of the US administration to its National Broadband Plan is confirmed, the situations in the countries under consideration will level out in the future, though a weak policy on network neutrality may delay this process. The Creative Contribution, as we proposed it, represents $12-16 \%$ of the subscription costs to fixed line broadband Internet. We will discuss below its potential impact on access to the internet for poorer households, but in general, this cost is small compared to the benefits and legal security it provides.

To illustrate why the contribution is not an excessive burden, it is interesting to compare it to household expenditure for mobile communications, the household budget item that has grown most explosively in the past 15 years. In France, the average household expenditure for mobile communications is $€ 72$ per month, and it has been shown that the impact of this spending has led poorer households to harmfully limit their spending on food. ${ }^{6 \mathrm{I}}$ In the US in 2007 , the average consumer unit spent more than $\$ 50$ per month on cellular phone communications, three times more than in 200I. ${ }^{62}$ This spending has kept growing since at a frantic pace, and has probably reached levels similar to France. The increased spending on mobile communications has more than erased the decrease in fixed line communications spending. It is a tribute to the enthusiasm of consumers for mobile communications, but also a clear sign of a major world-wide competition policy failure. It is particularly relevant to the discussion below of the impact of the Creative Contribution on access to the Internet for poorer households, as poorer households spend a much bigger share of their budget on mobile communications than on other ICT and culture-related items.

The Eurostat Pocket Book on Cultural Statistics (Eurostat 2007) reports that the average cultural spending for the first quintile (the $20 \%$ poorer households) was about 50\% of the global average spending in 1999 in Germany and $55 \%$ in France. (Maresca et al. 20II) remark that for specific categories such as blue collar workers, the spending is only $45 \%$ of the average. This means that the potential burden of the Creative Contribution on this category of households, while still 
representing less than $8 \%$ of their cultural expenditure, is significantly greater than for average households. However, the benefits afforded to these households in terms of access is also significantly greater than for better-off households. The poorer households, whose members often also have a lower level of education, are conscious of the essential role of the Internet in access to knowledge and education: the poorer households with children have a much higher equipment rate than the others. ${ }^{63}$

If one feared an adverse effect of the Creative Contribution on the ability of poorer households to access broadband Internet, two approaches could be followed to prevent it. One is to exempt poorer households, or make the contribution itself income-dependent. Nothing prevents a mutualized financing to be based on differentiated payments according to income, for instance, provided that the power of each person to influence who gets rewards or financing (and how much of them) remains equal. The second approach is to have a state, regional or local policy to cover the costs of the Creative Contribution for poorer households as part of Internet access or social and cultural inclusion policy. (Fisher 2004, pp. 216-217) considered a third approach, which is to make the household contribution part of income tax, but rejected it as too unpopular. For us, this third approach would also be contrary to the essence of the Creative Contribution, which is not a tax to fund public actions, but a pooling of resources within a society where all are contributors. The first approach, of a progressive contribution, may also raise some issues: the contribution will be collected by Internet Service Providers, which are not necessarily the organizations we most wish to know about our income. Furthermore, income progressiveness for minor payments is inefficient: it is much preferable, from a wealth redistribution point of view, to have a strongly progressive income tax (unpopular perhaps in some circles, but certainly needed) and to keep small statutory payments at a flat level. 



\section{Sustainable financing for the commons}

In the previous chapter, we defined what could be the initial regime of the Creative Contribution. We now reflect on its evolution in space and time. No doubt surprises will crop up in its implementation: how should it adapt to them? How should it be reviewed to address new challenges, in particular of scale? Can it be put in place in some countries first and others later?

As part of this exercise, we introduce a new way of looking at financing schemes that link the monetary economy to the non-market commons. The relevance of such schemes goes well beyond the cultural or information domain. They address a central problem: in the information age, how can we empower the members of a society to manage resources for the knowledge, environment and social commons?

\subsection{Evolution of the Creative Contribution in one country}

The reader might be wondering why when (Oberholzer-Strumpf 20I0) rightly stress that cultural sharing is a global phenomenon, we propose to manage resources for the financing of creative activity in national frameworks. There are good reasons for this initial choice: national frameworks are currently the only ones in which financial resources can be collected on the appropriate scale, the governance of the collected sums can be democratically controlled, and the debate on what is socially acceptable or not can effectively take place. It is a compromise, and we welcome the progressive development of resource pooling in other domains, at the level of global regional entities such as the European Union (a natural umbrella for more global schemes) or Latin America, or even globally in the field of health ${ }^{\mathrm{I}}$ or the environment. Nonetheless, in fields such as culture and creativity, mature democratic arenas are presently lacking and must be developed before large-scale resource collection can begin. In parallel with national mutualized financing schemes, grassroots initiatives such as Flattr, an intermediary for the voluntary pooling of rewards to creative works set up by Peter Sunde, one of the founders of The Pirate Bay, or the participative funding and co-production intermediaries already mentioned in section 7.3 will continue to explore the route of global resource pooling. 
Within the limits of a given national territory, two relevant parameters will evolve: the sums collected, and the financing needs. The number of broadband Internet users will keep growing, though in some countries it will reach a plateau, because a fraction of the population will never become connected. There will be a small minority of dissidents (people who refuse the constraints and blessings of connectivity, which is a legitimate right), and a number (somewhat larger, unfortunately) of persons who live in conditions that are not compatible with access to the broadband Internet. The development of broadband mobile Internet, if it steers clear of discriminatory models favoring the transmission of some contents over others, will open new avenues of development. The payment of the Creative Contribution can be shared between fixed line and mobile broadband users, but the same individuals or households will ultimately be paying. Assuming a fixed level of contribution per household, the sums collected can be expected to grow by a further $20-30 \%$ in developed countries. ${ }^{2}$

Let's now turn to the financing needs. Two of the choices we made when estimating them initially might need to be reviewed later. For rewards, we selected the number of people who should be rewarded at or above a given minimum level; for the financing of new works, production and the environment of creation, we selected an initial amount corresponding to $5-7 \%$ of all investment in producing new works in all (digital and analog) media today, plus an additional I0\% of this amount towards the creation and maintenance of environments contributing to the early recognition of quality works. ${ }^{3}$ What if not enough potential rewardees show up? What if, on the contrary, the many-to-all cultural society model installs itself more quickly than we expected, and people who should be rewarded (in the opinion of many) are not, because of the limits we have set for the system? What if orphan activities in production prove to be a much wider domain, and despite the many other mechanisms that can contribute to finance them, one wants the Creative Contribution to play a bigger role there?

One can handle these uncertainties using three tools:

- Incorporating from the start, in the legal basis of the Creative Contribution, a plan for it to reach its set value only progressively over three years. This is probably necessary so that the collected resources are efficiently used. Those who stick to a compensatory approach will complain that the right to nonmarket sharing is going to apply immediately, while the financing side will be progressive. This is mistaken, because file sharing already exists, and the impact of its legal recognition ${ }^{4}$ will also be progressive. Of course, this impact will not necessarily be negative for established players or sources of production investment, but even it if were, it will take time to build up. More generally, the governance mechanisms that we discuss in chapter 9 should include enough flexibility for a smooth adaptation of, for instance, the share of rewards allocated to various media, and sufficiently strong democratic govern- 
ance guarantees to minimize the risk of specific interest groups being the chief drivers for this adaptation.

- Planning, again as part of the law, a review of the mechanism five years after it comes into force. Such reviews are inserted in an increasing number of laws, but in many cases, they are a formality, and not always observed in some European countries. For the issue at stake, however, the public debate can be expected to be lively enough for the review to be a real one.

- Conducting, prior to the finalization of law proposals, a societal and stakeholder debate on the use of less-than-proportional reward functions. As pointed out above on page 96 and detailed in appendix B, they are a key instrument of distributive justice, and a management tool with which one can better handle the uncertainty surrounding the initial evolution of popularity distributions. Those who believe that the present observed commercial popularity distribution is the product of individual preferences and a perfect cultural market will no doubt complain. But the debate will enable other views to be aired, describing the shape of commercial popularity distributions as having more to do with the strategies of a few commercial actors and a very imperfect media market.

\subsection{International aspects}

Does it make sense to implement a scheme such as the Creative Contribution in a single country? A quick and easy answer is that it will prove so attractive that it should rapidly spread to other countries. In Europe, the idea has already propagated nicely. With some variations, proposals exist in Germany, Belgium, Sweden, Italy, the Netherlands and even in countries where statutory resource pooling is rare, such as the UK. It is being discussed elsewhere: in the US and Canada, of course, but also in countries such as Brazil. ${ }^{5}$ The reality will propagate even better than the idea.

One country will have to be the first. Let us call it country A, and place ourselves within it. How does usage in other countries interact with the Creative Contribution, or a similar mutualized financing scheme associated with a right to non-market sharing of digital works? First, we consider upload from other countries. Internet users in country B will naturally be inclined to exploit the recognition of non-market exchanges in country $\mathrm{A}$, and the resulting existence of quality services in that country: ${ }^{6}$ they will make the works they have access to and like available via IP addresses located in country A. If the persons who have a claim to the rewards for these works are represented in country A, they will benefit, as they would from any export mechanism. The act of uploading from country B will remain illegal, but the courts in that country will have to take into consideration the added income possibly collected by those who claim damages. 
It will of course be more difficult to control media chronology across multiple countries. However, the ability to control media chronology across borders is already very much undermined not only by unauthorized sharing but also by legal decisions, such as the recognition of the legitimacy of de-zoning for DVDs. ${ }^{7}$ In reality, when the Creative Contribution is generalized, it will create an equivalent of something that has been the dream of all digital commerce, but which is impossible to implement in a commercial context: a form of differential pricing, which can be termed differential contribution. Because the amount of the Creative Contribution is set while taking into account purchasing power parities (PPS) or another formula for adapting to differences in income, people in various countries will all have access to the commons of sharing while contributing an amount depending on the local wealth. This amount can and probably should be zero in the poorer countries.

More complex problems could arise with downloads from other countries. As with current on-line file sharing, whether legal or not, there will be no way to prevent users from country B from accessing works made available in country A. ${ }^{8}$ This leads to two questions:

- Does this go against the interests of creators in country A? It depends on what one compares this eventuality to. Certainly not compared to the present situation: a greater degree of international visibility (usually very limited, for the vast majority of productions) can only lead to increased export sales proceeds for works created in country A. Only a small fraction of works, those are already massively exported, could conceivably lose revenue, though even that remains to be proven. The American example should serve to highlight the immense commercial and cultural influence benefits of exposing other countries to one's domestic production freely and massively. On the other hand, country A would be justified in bemoaning the absence of a reward mechanism in country B, which deprives creators and other contributors of the returns they would get if such a mechanism was in place.

- Should this type of usage count when allocating the collected license fee amounts? This question cannot be answered conclusively without a more detailed study, but it seems preferable to avoid it. Although it would reflect the full usage patterns, the usage measurement mechanisms we propose ${ }^{9}$ would be harder to operate reliably in an asymmetric context, and it would depart from the idea of reward and resource pooling organized in one society.

\subsection{Economy and non-market commons}

The development of information technology and universal networks creates new spheres of non-market exchanges. This leads to a de facto demonetizing of some activities that were previously the object of monetary transactions, or creates a 
dual system where the same contents are accessible in both manners, through commercial transactions (generally with some added-value in comparison to non-market access) and by exchange between individual peers.

Scientific publishing is an example of a domain which is in the process of becoming entirely non-market on the access side, even though some publishers still cling to the belief of remaining monopoly dealers. As a result, it has to search for financing mechanisms for those parts of the scientific publication that remain essential to its aims: editorial selection, limited but real production costs (in particular when paper publication is still deemed useful) and post-publication processes such as commenting and revision. As most research work being published is publicly funded, the natural financing solution would be to make these publishing costs part of the research funding. Part of this solution was implemented by the European R\&D funding programs, when they decided to allow authors to claim back publication fees. However, the fees are reimbursed whatever their cost, and proprietary journals often charge three times more for their "open access" option (where the author pays to ensure access to their publication is open) than journals with open publishing models. This amounts to a significant public subsidy for proprietary models and is clearly unacceptable. Aside from this debate, there is also a need to provide complementary financing for the publication of results from unfunded or insufficiently funded researchers, and researchers in poorer countries or institutions. This has been a test case for pooling resources for the creation of knowledge commons. In the case of the Public Library of Science, ${ }^{\mathrm{IO}}$ it is handled by a combination of support from foundations (in particular the Open Society Foundations) and donations from individuals. This solution will probably not scale up as fast as the needs it covers, as open publishing is increasingly becoming the dominant model.

In the cultural domain, direct public funding is less dominant: though it plays an important role in financing creative activity in many countries, it is indirect for a large part, a typical example being the employment of artists in public educational organizations. In fact, there would probably be strong opposition to a predominant funding from governments towards, say, the expression of individuals in the public sphere, or towards news media, for fear that it would lead to forms of censorship or influence on contents. It is thus natural to seek a direct societywide pooling of resources, where the role of government is to set up the system and to act as a trustee for its transparency and democratic governance. It is the route we have followed with the Creative Contribution proposal, which, as an economics construct, is best described as a government-initiated society-wide pooling of resources for the conditions of existence of the cultural, expressive and information commons.

It turns out that similar situations of having to finance the conditions of existence of commons (by definition outside the scope of market transactions) are also found well beyond the domain of information and culture. For instance, while local community action can do a lot for managing environmental resources, 
some need to be maintained on a more global scale. One such example is the preservation of biodiversity among plants and genetic resources of agricultural or medical interest. The industrialization and standardization of agriculture have led to a strong reduction in biodiversity, directly through the reduction in the number of cultivated varieties, and indirectly through the effects on natural biodiversity of agricultural landscaping, weedkillers and pesticides. In reaction, movements for a better preservation of vegetal biodiversity gained momentum. Two - probably complementary - forms of actions for this preservation exist: preservation storage of vegetal varieties in seed banks, funded by governments under the umbrella of international organizations, and the decentralized in situ reproductive management of biodiversity by farmers. ${ }^{\text {II }}$ Government action has, for a long time, deincentivised the latter by installing costly certification procedures for seeds, whose requirements stood contrary to in-situ biodiversity preservation ${ }^{\mathrm{I} 2}$ and by requiring certification for the commercialization or even the non-market exchange of seeds. Fortunately, grassroots farmer and amateur gardener efforts countered these trends, and we recently witnessed a return to a larger set of varieties in particular for fruits and vegetables. Pharmaceutical and cosmetic companies as well as agro-food companies themselves are now conscious of the value of global biodiversity preservation, even if they would rather free-ride on it, proprietarizing its most valuable components when industrializing them. There is a clear need to incentivise the in situ preservation and development of biodiversity, which turns out to be an activity to which many can contribute and from which all benefit. Some forms of this activity (biodiversity inventories, home gardening) have their own motivations and do not need further financial incentives. Others, such as the reproduction of seeds through the culture of cereals and other plants are costly and a societal pooling of resources would be useful.

Similar situations also arise in the management of social public goods such as health: everyone can contribute to a better knowledge of health conditions or problems encountered in the use of drugs. Furthermore, large-scale pooling of resources to search for treatments for rare or neglected diseases is already a reality, bringing together individual donations, governments and private funds.

A specificity of information and knowledge commons is that the financing schemes they require must not create transaction costs within the flows of information and knowledge. In this sense, they are "purer" commons than physical commons or social public goods, where some transaction costs in economic incentives are acceptable, because there exist anyway other transactions to which they can be attached.

Within the landscape we have just sketched out, a new vision of schemes such as the Creative Contribution arises: they can be seen as, and could actually become, a healthy form of specialized currency creation. To see how this works, let us assume that we have an idea of how much new investment occurs every year to produce components of cultural commons in one country. We then divide this 
amount into equal parts for each individual, and distribute (virtually) the corresponding sum to each person above a certain age, empowering them to allocate it according to their usage (to reward works) and their preferences (to finance production). "Hang on a second," you might say. "The Creative Contribution will come out of our pockets, not out of some virtually distributed pot of money that we receive." This is true at the moment, but the alternative model we just outlined might be the one of the future. For it to become acceptable, there is one condition: the resources must be pooled among a community of peers, and the amount that leaks out of the system (e.g. in speculative investments) must remain limited. The annual distribution of individual credits must not lead to monetary inflation. Inflation would rise if too much of this currency ends up distributed to some individuals or organizations, over and above what they need to live on and to put together meaningful creative projects. 



\section{Implementation}





\section{Organization and complementary policy measures}

The best proposals can turn into a bureaucratic nightmare, or fail to serve their intended aims, if their implementation is inadequate. The challenge is made harder by the fact that organizational issues are no fun. Internet users and creative people (two very much overlapping categories) are not particularly keen on creating organizations, specially not when they have to deal with the large-scale management of money. They often create ad hoc organizations that handle the complex logistics of a project, or art and advocacy collectives. They are often entrepreneurs, engineers of these lightweight virtual corporations which have recently received some legal recognition in a Vermont law (Bollier 2008). But sitting on steering committees and management boards is not the activity of choice for most of them, and they ignore or outsource (when they can afford it) most of the interface with collective management organizations, except to complain about the misgovernance of the latter. To involve Internet users and a much wider set of producers and contributors to works in the management of the Creative Contribution, we will have to use innovative means.

A non-fiction writer and analyst must also show some caution here. Organizations are not created, or even designed, by writing books. The organization of the Creative Contribution will differ from country to country. Moreover, putting it in place must be the first step in a participatory process, not the execution of a set plan. To cope with these challenges, we limit this chapter to the description of the key components that will have to be present in any organization for the Creative Contribution or a similar system. We then outline how the governance of a particularly difficult decision (the split of rewards to different media) could be handled. And, finally we stress the need for a number of complementary policy measures that would maximize the benefits of the Creative Contribution.

\subsection{Principle and essential components}

Let us leave aside legal aspects such as the recognition of the right of non-market sharing of digital works between individuals and the reaffirmation that providing means of doing so is also legal. The system of reward and support to creation embodied in the Creative Contribution is not based on copyright. It is an autono- 
mous recognition of the social rights of creators and users in the information technology and Internet era.

Government and parliamentary financial management and governance overseeing

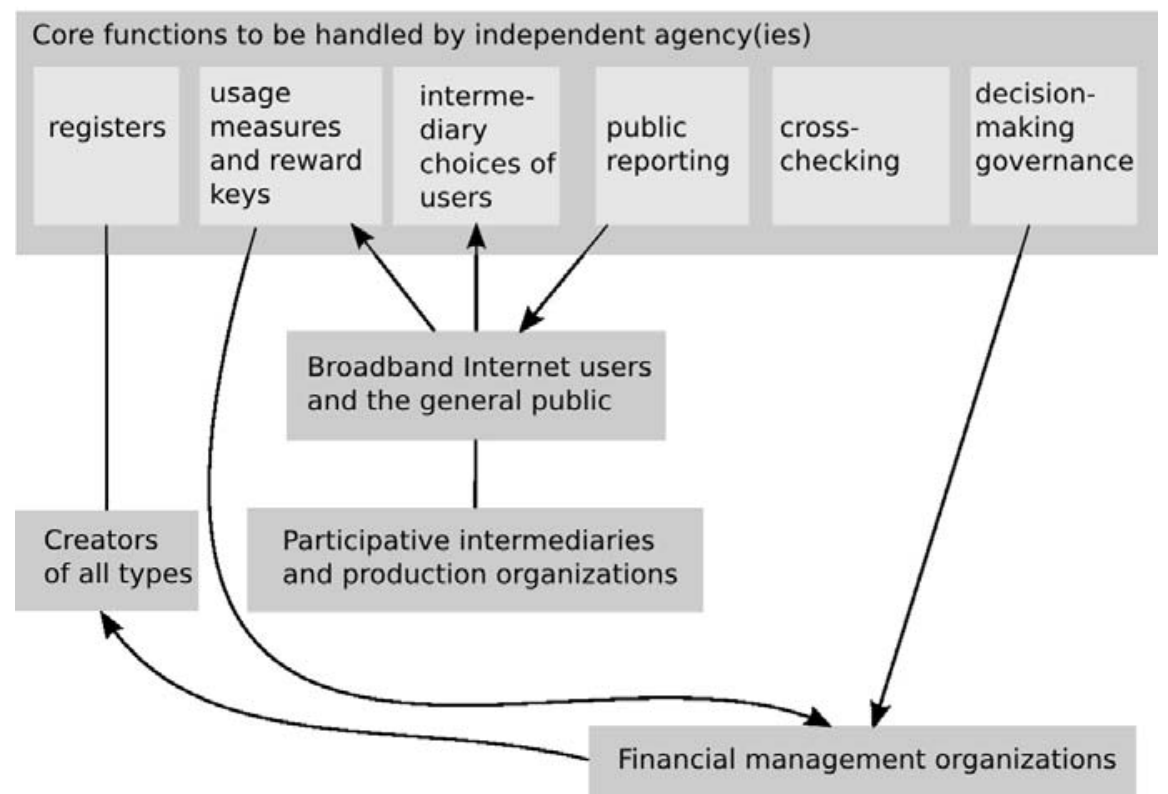

Fig. 9.1. Core components for the organization of the Creative Contribution.

What are the necessary components for the management of our financing system? We identify here seven essential functions that can be implemented within a single organization or several. Whether a single organization is put in place to perform these functions, or they are shared among multiple organizations, each function can only be trusted to an independent public agency, set up by law, and which is accountable to government, parliament, and the public. The seven functions are:

- The interface with broadband Internet Service Providers, the reception of the funds collected by them, and their transmission to financial management organizations. This function is not represented in figure 9.I, as it raises no particular difficulty.

- The operation and maintenance of public registers of potentially rewarded works and their contributors (see section I0.2), as well as of intermediaries 
that are potential recipients of the support to production and to the environment of creation.

- The collection of usage data by means detailed in section I0.3 and the estimation of the gross rewards for each medium (which depend on the split between media). These distribution keys are then transmitted to the financial management organizations.

- The collection of broadband Internet subscriber preferences for the allocation of the support to production and the environment of creation, as detailed above on page ro4.

- Cross-checks and other measures to prevent and detect fraud, as well as periodic surveys of large samples of users in order to improve understanding of the usage of Internet contents and their production (see chapter Io).

- Ensuring that the public has access to the data and detailed reporting on all aspects of the rewards and support to creation. This is essential not only for transparency, but also because this public access ensures that citizens and third-party organizations can help detect any fraudulent registration of works and evaluate the fairness of the rewards.

- Steering the participatory decision-making processes for decisions that cannot be preset in legal texts and must thus be taken during the course of the implementation of the Creative Contribution. We discuss these governance processes in the next section, taking the particular example of the split of the global amount of rewards among media.

An original aspect of this organization is that it will rest on one or several public agencies receiving the essential data they have to process from the general public, and reporting to it as well as to government and parliament.

We have used the expression "financial management organizations" as a placeholder for the type of organization that will actually distribute the rewards within one medium. We leave open here the question of whether existing collecting societies could fulfill this role, or whether new types of organizations will be needed. The first option would require collecting societies to reform their governance considerably, and provisions would have to be made for an airtight separation between the sums collected on the basis of copyright and the new rewards.

The cost of operation of the overall organization is discussed in the next chapter (section I0.5).

\subsection{Decision-making and democratic governance}

In the past 30 years, policy decisions regarding access to and production of culture have raised serious concerns about democratic governance. Despite accumulating evidence that these decisions affect each of us, they were prepared in consultations with a restricted set of industry players, where the dominance of the 
large content industry was supposed to be balanced by the presence of information technology companies. Citizens and consumers were at best informed or allowed to provide a few position statements, but proposals originating from them were never seriously studied. As an example, the latest rounds of repressive laws against file sharing were met with public furor, particularly in Europe. The refusal to even discuss or study alternative financing frameworks permitting the recognition of file sharing fueled this anger. The new organization put in place to manage the Creative Contribution or similar schemes will have to prove that it can do better, that it can organize truly open decision-making processes involving multiple stakeholders, and duly take the views expressed into consideration.

The distribution of the global amount of rewards among different media is an example where such a debate will be required, which we can use to illustrate the conditions for it to be fruitful. First of all, options should be truly open, including the option of treating all media in one global basket. There are reasons for doing so: all media are mixed in the real practice of Internet usage. Also, in economic terms, the cost of a copy of a work in various media (where there are economic reference points) are not so different. A musical album, a film on DVD, a book, a photographic album, or their digital equivalents are all in a similar price range, despite differences in the cost of production of an initial copy, or in the time spent using each. If the various media interest groups accepted such a solution, it would no doubt be the best, as it would lead to a simpler process for the measurement of usage. Another option, which we already mentioned, would be to let broadband Internet subscribers make the decision themselves, by allocating percentages of their contribution to this or that medium. Yet another option would be to look for some ground truth proxies in the measurable usage patterns of various media. Many parameters should be considered when making the decision, in particular the number of popular works which are or are not registered for rewards and the portion of the total which they represent.

All stakeholders should have a voice, and their legitimacy must be properly acknowledged: private interests, citizens, advocacy groups, academic researchers, policy analysts... When the decision-making process reaches the Cui Bono ("for the benefit of whom") question, these voices are not all equivalent - policy should ultimately be designed for the benefit of citizens - but all of them should be heard on an equal standing. Of course, there are practical constraints: it is easy to organize multi-stakeholder meetings between representatives of incorporated organizations (including advocacy groups), academic experts, government and agency staff, but involving individual citizens in the decision-making process calls for entirely different procedures. Internet-based participatory democracy is an obvious candidate for the organization under discussion.

Finally, at some point decisions must be made: endless debate is not an option for an organization which has funds to distribute. A clear and explicit decisionmaking process must exist. In the case of an independent public agency, this 
means that its management must be empowered to make these decisions according to its mandate, provided that they are justified on the basis of both the inputs received and the objectives of the organization. In turn, this means that the mandate and the statutes of the organization(s) will have to be drafted carefully.

Citizens will also have a role to play in evaluating the activity of the organization(s), in addition to the major contribution they make to its activities by providing data on usage and preferences.

\subsection{Additional policy measures}

The Creative Contribution will carry its own direct benefits. These benefits will be maximized, however, if attracting attention in the non-market sphere can also lead to increased commercial revenues, when desired. In this section, we outline a number of policy measures that could contribute to a better synergy between both domains.

\section{More open markets for cultural goods and services}

The markets for cultural goods and services are presently characterized by an extreme degree of concentration. The large media publishing and the new Internet providers have a strong control over the distribution of works and the provision of services. We presented an overview of the degree of media concentration in the Oligopoly box on page $6 \mathrm{r}$.

Some of these concentrated distribution channels, in particular in digital distribution, are more open to offering a large set of titles for sale. However, there are worrying signs that this theoretical openness does not lead to a more diverse market because of a combination of heavy promotion over a short period, DRM that are de facto imposed by publishers on authors, and access mechanisms on platforms which disfavor intermediate popularity titles. Some of these problems will be solved with the recognition of the right to non-market sharing, but a basic competition policy could help limit others, whether by anti-trust, dominant position abuse or preventive competition policy, such as an open standards policy.

\section{Fair trade requirements for commercial offers}

One of the positive effects of information technology and the Internet has been the emergence of fair trade offers in the content markets. In particular, some providers guarantee that a significant share of the revenues will actually go to the artists. These offers are presently confined to niches, as they are the object of an unfair competition from classical offers, who should rightly be re-branded "unfair trade" in contents.

Minimal requirements of fairness (to authors and other contributors), taking in account the different structure of costs for digital media, are a real policy option: they can be attained through voluntary self-regulation under the vigilant eye of the 
public, but also through incentive or normative policy. Contract law could be important, for instance forbidding the common practice of deducting promotion actions from the accounts of authors and other contributors.

\section{Strong and effective requirements for network neutrality}

In the past two years, network neutrality, that is the equitable transmission of bits on all types of networks, regardless of their origin, contents, or the application and protocol used, has become the hottest policy issue in the digital environment. ${ }^{\mathrm{I}}$ Governments have taken various positions. The Federal Communications Commission voiced a strong commitment to network neutrality in the US, but it has yet to be translated into effective measures, and it faces significant opposition from cable networks and mobile communications operators. European governments, under intense lobbying by telecommunication companies intent on vertical integration with premium content publishers, ${ }^{2}$ tend to tolerate discriminatory practices, with weak and ineffective guarantees of consumer information. The Google/Verizon agreement, analyzed by (Zittrain 2010), and the fuzziness of some of its clauses, show that even a company like Google, whose success is built on network neutrality in the general Internet, is prepared to accept network discrimination to promote its own side interests.

If demanding and effective standards of network neutrality are not adopted, new forms of gatekeepers will replace the old ones and maintain artificial scarcity despite the abundant capacity of networking. They will produce networks with which they will "canalize" the Internet, to make it function like stores with limited shelves, or media with a limited editorial space. The rest of the traffic will still be there, but it will be discriminated against in terms of quality of service or even in terms of accessibility. The potential of the Internet for a diverse culture will have been lost, or at least damaged. Network neutrality may seem an abstract concept, but the lack of it will be felt in very concrete ways.

\section{Encouraging sharing-compatible commercial services}

The idea here is not to promote a specific form of business, but to maximize the compatibility between non-commercial sharing and commercial offers. It has a bearing on the detailed definition of the scope of the Creative Contribution. For instance, news media could offer their daily contents only to subscribers, but permit free sharing of archives. Journalists, or their employer (depending on laws and contracts) could then benefit from Creative Contribution rewards for this sharing. This can be seen as a reward to the service rendered to the cultural commons within the frame of a private for-profit organization.

\section{Keeping advertising pollution under control}

Advertising is a debated issue in the Internet world. Initially, advertising offered means to obtain revenues from delivering a limited value to many users, which is 
a typical situation on the Internet. However, advertising is highly polluting. Even schemes such as AdSense, which have been designed to avoid the need to concentrate attention on a limited set of contents (that plagues classical advertisingfunded media), have a polluting effect. A blog writer using AdSense will write for two public instead of one: readers and advertisers.

One must draw lessons from those media that managed, at least for a period, to provide access to information of a reasonable quality whilst being partially financed through advertising, in particular newspapers. This fragile balance did not materialize spontaneously: it was the product of regulations (on signaling advertising), of ethical codes (of independence of the editorial contents, or bans on product placement), of internal and external counter-powers. On the Internet, technical innovations can also play a part, with ad-blocking software. All of these attempts to resist pollution by advertising are under threat, but they can be regenerated if authors and consumers start refusing this degradation of media quality. 



\section{Usage measurement for equitable rewards}

We are close to our intended goal of making a reasonably complete and self-consistent proposal. We have one final question to tackle: can we really measure the non-market use of works precisely and reliably enough to set the basis for rewards? Strongly divergent opinions have been expressed on this topic in the past. Some managers of collecting societies, who were hostile to a flat-rate-based legalization of file sharing, initially claimed that it was impossible to measure usage, and that the system would be prone to an enormous amount of fraud. ${ }^{\mathrm{I}}$ Then, as such a system started looking increasingly likely to be implemented, others said that there was no problem at all, provided they were the ones to do it. ${ }^{2}$ Finally, yet others declared that it was intrinsically unfair, since the methods would necessarily use statistics, while their present measures for other sources of revenues use detailed counting of every use. ${ }^{3}$ This objection is ironic, since they measure only an extremely limited fraction of the use of works, compared to what we are trying to address.

These disparate arguments further motivate us to evaluate the precision which can be achieved in practice. We start by describing the structure of a possible measurement system, then detail some of its aspects and describe what it can achieve in realistic conditions. Appendix $\mathrm{C}$ details the underlying model supporting our claims.

\subsection{A general usage measurement system}

\section{Structure}

(Fisher 2004, pp. 223-234) described a potential measurement system for rewarding artists. It was mainly based on large-scale sampling, but also included alternative or complementary possibilities, such as using the direct expression of preferences (for example, genres). We also favor a sampling system, leaving the direct expression of preferences for the financing of production. As Fisher did, we note that a fair reward system does not need to track every use of a work, but only to estimate the relative use of each work compared to the others to a reasonable level of precision. Our system is of a different nature from Fisher's and operates on a different scale. It is at the same time more modest and more ambitious. 
More modest, because we do not try to measure the value that users have derived from a work, but only whether they shared files and accessed or uploaded on-line contents. More ambitious, because we are addressing a bigger set of works and media than the classical music and motion picture entertainment domain considered by Fisher.

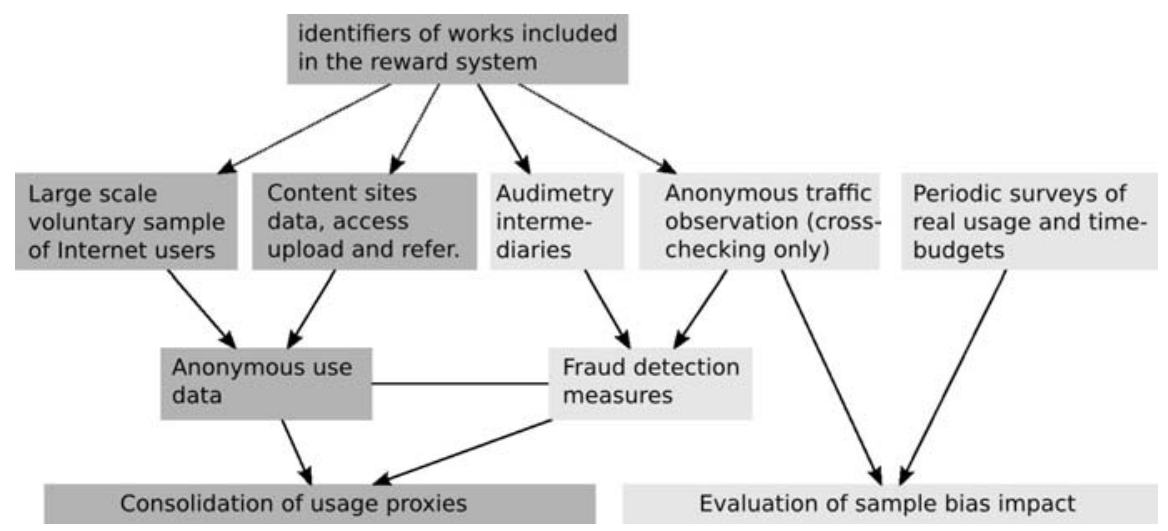

Fig. 10.1. General structure of a possible usage measurement system.

There are two main sources of measurement in the system: a large sample of voluntary broadband Internet users, and access and usage data provided by content sites. The first source is used for measuring the actual sharing of files, main object of this book. The second source is used to measure how users directly read, listen to or view contents on non-market sites, as well as how they upload and reference for recommendation on these sites. If non-market sharing of digital works is legalized and all broadband Internet users make a statutory contribution, Internet users and the sites they put together to make contents accessible are, considered as a whole, the best guarantors of a fair distribution of the rewards. Of course, some of them will possibly try to abuse the system, and other parties may disguise their efforts to do the same. We thus will have to put in place a number of security and cross-checking mechanisms to prevent, detect, and investigate fraud.

We have yet to specify what constitutes "usage", which data would be collected from the Internet user sample or from content sites, and how. For our purposes, usage is defined as any form of action involving publicly accessible contents that manifests interest for a work. In other terms, for file sharing, the usage we will measure is the entry into the possession by individuals of a full file representing the work, or the upload of the file on a publicly accessible content site (on $\mathrm{P}_{2} \mathrm{P}$ file sharing networks, access and upload coincide). For contents that can be used directly on-line, in particular blog entries and other contents produced for direct Web use, usage will be constituted by the display of the work in a Web browser 
(complete or sufficiently long for a time-based document such as video and recordings). Finally, public manifestations of interest, other than uploading a document for download on a non-market site, such as linking to or referencing will also be usable if this proves not to be too prone to abuse.

The position we defend here is that the data must not consider what individuals do with the work, once obtained, in the private sphere. Any system that would require even a sample of users to provide data about their personal usage of a work once they have acquired the file that represents it would be rejected by users, and would lead to the construction of an absurdly complex and probably inefficient use measurement system.

The fact that we collect possession, access or recommendation information (we call them usage clues) and not hard proofs of user enjoyment will no doubt be deemed unsatisfactory by some. We find this view misplaced. (Fisher 2004, pp. 225-226) remarked that when people download a music file, quite often they never listen to it or listen to it just long enough in order to decide that they are not truly interested, in many cases they listen to it only once, and only in a few cases do they listen to it repeatedly. Well that's also true of people who buy CDs (with different proportions). Many supposedly hard proofs of user interest or enjoyment in the existing copyright system are no better, or even much more approximate, than our access, upload and recommendation clues. Statutory licensing for radio, a scheme that presently accounts for a significant share of royalties for music authors and composers in many countries, is entirely statistical: it counts broadcasts unit by unit, but rates are based on broad classes of population able to receive radio, and in rare cases on statistics about the probable audience share at a given time of broadcast. ${ }^{4}$ Fees for public performance of music in stores or restaurants are collected from owners (who in turn charge customers as part of the general expenses they must recover in their revenues), while the existence of any enjoyment is uncertain. Clues are just an honest word for indications of interest, whose aggregate sum will be a valid basis for a reward system. If desired, one can give weights to various clues, but we suggest postponing this weighting until we have more experience and understand more about user practices on the Internet and about the way the reward system works.

Access and use data from content sites will be particularly useful for media where the number of creators and works is very large, such as blogs or photography (even when an entire personal blog or photo gallery is treated as a single work). Content site data is not to be trusted any more blindly than user-provided data. However, it is amenable to different forms of control, and importantly, it is exhaustive in its own realm. The independence between the two sources is also precious. For fraud detection purposes, the consolidated data coming from our two sources can be cross-checked against other data from independent sources that are hard to skew simultaneously: observation of Internet traffic and possibly data from audience measurement intermediaries. 
Last but not least, any usage measurement system must include surveys of a representative sample of Internet users, conducted periodically (every two years?) to monitor their non-market use practices. Considering the specifics of the domain under study, where rare practices can be important, the sample will have to be large, in the same range as for time budget surveys. It will provide very valuable information on Internet use practices which are not connected to commercial transactions, and thus poorly understood at present. It will be possible to use the results of these surveys to evaluate the bias introduced by the voluntary permanent sample, and by other technical aspects of the reward system. This does not mean that this bias must necessarily be corrected: it is not absurd to give a higher weight to the use patterns of the voluntary participants in the sample, provided that participation is open to all. The same is true for data resulting from the use of user-generated content sites. It can be seen as a form of reward to the rewarders, which is in the spirit of a contributive system.

In the following sections, we detail some moderately technical aspects of the usage measurement system and discuss the precision that can be obtained. The system we propose is by no means the only workable one, and it is open to further discussion, but it is important to show that there exists at least one approach that can meet the requirements of precision and resistance to fraud.

\section{The requirements for a usage measurement system}

Since our measurement system produces the data used to compute the level of rewards, it must be precise enough for these rewards to be equitable, in particular for rewards that represent more than a symbolic amount. We address this issue in detail in section ro.4 using a model described in appendix C.

One common argument against the idea of rewards for the use of digital works in file sharing is that it would be prone to fraud. We address this issue also in the sections where we discuss precision, as both aspects are linked. It is useful to describe here the philosophy of our approach to fraud for such a system.

In a recent paper, Andrew Odlyzko, one of the key analysts of Internet issues, has discussed the joys and pains of designing secure systems that use insecure means (Odlyzko 2010). He proposes two approaches, both of which we will use: slowing things down and cross-checking independent sources. Slowing things down is a pain, when the beauty of digital technology and the Internet is that provision of data can be fast and seamless, but we will not be able to avoid some of it. Just like any website needs precaution against spam robots, we will have to require human confirmation at some key stages. It will be the case for the registration of participants in the voluntary sample, and for the periodic reporting of consolidated use clues by members of the sample. Cross-checking is the key to the prevention, detection and possible investigation of fraud. It creates some complexity in the overall design, but no digital measurement system can avoid it. Fortunately, some widely used software such as anti-spam plug-ins for the free- 
software WordPress platform provide very useful inspiration. ${ }^{5}$ The technology to be put in place will have to be safer, as the benefits of possible fraud are more immediate that those of spam. No system will be safe forever, they will have to be revised in face of new techniques and approaches to fraud. Despite all this, one should not overestimate the level of fraud in social systems in general, and information technology systems in particular.

Contrary to a common ideological bias, fraud in social systems such as the one we propose does not frequently emanate from ordinary recipients. Most abuse originates with established economic players, and most fraud comes from organized networks. A typical example is the unemployment benefit system put in place, in France, for workers in the live performance and creative activity sector, called régime des intermittents $d u$ spectacle. ${ }^{6}$ When the total cost of the system increased, fraud and abuse were invoked among possible causes. Independent studies were conducted, which concluded that the system was indeed being abused, mostly by large media companies, in particular broadcasters, including - ironically - public broadcasters, who disguised permanent employment as intermittent in order to receive subsidies from the system. Direct fraud by recipients was found to be insignificant, even though people received benefits for activities whose inclusion in the system was not originally intended (Corsani-Lazzarato 2008). There was also criticism against the high-level benefits distributed to some movie actors. Similarly, fraudulent use of social and health insurance benefits by ordinary recipients has been shown to have a limited relative financial impact (CAF 2010).

In order to be accepted, the usage measurement system must not install controls which would be perceived as a presumption of fraud, but it must not be naive either: it must be capable of detecting and - if possible - of preventing fraud if and when it occurs. No system is perfectly resistant to fraud. Existing copyright rewards are no more exempt from fraud than VAT, for instance. The reward system we propose won't be either, but one can make fraud easier to detect, limit economic incentives for it, take various measures to eliminate fraudulent data from measurements, and prosecute fraudulent behavior. As rewards are based on comparative levels of use, the best protection against fraud will come from the sheer mass of non-fraudulent data, but as fake data can be produced at will, we must make sure that it will be hard to inject it in the system.

\subsection{Registration and identification of digital works}

A reward system cannot function without some way of identifying works that are shared. When trying to detect unauthorized file sharing, copyright holders and their private contractors use automatic recognition systems. Though much progress has been made since we researched these subjects in the I9gos, automatic recognition systems have intrinsic limitations in terms of cost, complexity and 
how many works can be reliably recognized. ${ }^{7}$ In the application of the French HADOPI law, the representatives of right holders track use of 10,000 musical tracks and I,00o moving image documents, a ridiculously small subset of our culture, although one which makes it clear in whose interests they are acting. User-oriented applications such as Shazam ${ }^{8}$ offer recognition of up to 8 million music tracks from ten second samples of audio for smart-phone users. Though we do not have data on their exact recognition performance, such systems clearly accomplish an impressive feat. However, this kind of gymnastics is not needed for our purpose: in the context of a reward system for a legal activity, there is fortunately a much simpler approach: let the producers of works identify them voluntarily, by registering and tagging them.

There are various traditions regarding registration of works. In the US, registration of works was a condition for obtaining copyright protection until the Bern Convention Implementation Act of 1988 , which came into force in $1989 .{ }^{9}$ The US Copyright Register contains 6r million entries, of which 16 million are in a digital database. By contrast, European countries have not made registration of works a condition of author rights or copyright, even though some of them have compulsory legal deposit laws. The system of registration we have in mind here is of a different nature, and is very similar to the system described in (Fisher 2004, pp. 203-205). It can be kept to the minimum needed for the administration of a reward system, namely:

- identification of the registrant;

- certification by the registrant that $\mathrm{s} / \mathrm{he}$ is the single creator (in the sense: author, performer or technical contributor benefiting for neighboring rights) for the work, or has been authorized by other co-creators. A presumption of entitlement ${ }^{\mathrm{IO}}$ will apply: no check nor examination of the validity of the claim will be done (see below);

- identification of the creators and other contributors to the work and of their respective share (see below);

- provision of a Uniform Resource Identifier (URI) pointing to a reference copy or location of the work available on the Internet (an exemplar in the modern sense);

- attribution of an unique identification code which, as we will see, can be closely tied with the URI.

In countries with a registration tradition such as the US, the corresponding register is a natural choice to act as the registration agency. In other countries, it will have to be a new organization. The register must be public and publicly searchable and downloadable, in order to make it possible for creators or their representatives to check that their works have not fraudulently been registered by others. In all cases, opposition procedures must be possible, and associated sanc- 
tions against the abusive registration of works must exist. The registration process should be a matter of minutes using the Web. If it takes any longer or if there is a transaction cost, it will represent a harmful obstacle to the diversification of rewardees. In contrast to (Fisher 2004), we think registration should be free of charge and funded as part of the management costs of the Creative Contribution, which are evaluated in section I0.5. Mass registration should also be possible, for instance for the works already included in a copyright register, or for works registered with collecting societies. However, in both cases the shares allocated to various contributors will have to be treated with caution, as this information can not be readily transferred from other forms of registration: it depends on specific legal provisions and/or the contract provisions described above on page I20.

It should be noted that the registration described in this section is not a formality in the sense prohibited by the Bern Convention article $5.2:{ }^{\mathrm{II}}$ it is not a condition for obtaining copyright, nor for proving one's rights in a copyright procedure.

It is worth considering in more detail the unique identifier and the information associated with it. An international standard exists that us widely used, for instance in the context of scientific publication archives: the DOI or Digital Object Identifier (Paskin 2008). In parallel, many other systems exist for specific categories of published works, such as the ISBN ${ }^{12}$ for books. The characteristics of the DOI make it suitable for use in a reward system for a very diverse set of works. It is agnostic with regards to the nature of works. The production and management of unique identifiers can be distributed among various agencies. The DOI permits a durable connection between the identifier and a resource identifier (URI) pointing to a reference copy or location on the Internet, which can be updated by the registrant as and when necessary. Identifiers can apply to different levels of a composite work, enabling the definition of a single identifier for a blog or a personal photo gallery. This is important to handle the rewards to works that are composed of multiple units, each of which might not receive enough attention to qualify for the minimum level of rewards. This type of grouping can be applied only to works that are all accessible at a unique Internet location, and thus truly constitute an ensemble that can be rewarded as a unit.

The use of the DOI might raise some concerns from the point of view of being an open standard: some patents apply to its implementation. ${ }^{13}$ We nonetheless use the DOI as an example of a voluntary identification system that fulfills all the relevant functional needs.

Finally, in addition to the abusive registration of works, one possible form of fraud could be the production and dissemination of copies of works in which the digital identifier has been replaced by the identifier of another work. This can be prevented and detected relatively easily using cryptographic integrity checks. ${ }^{\mathrm{I}}{ }^{4}$ The exposure of the person benefiting from the fraud should also act as a powerful deterrent. 


\subsection{Data collection}

The large sample of Internet users providing anonymous use clues on a voluntary basis plays a key role in our proposed reward system. If a large proportion of Internet users participated in the sample, it could function efficiently as the sole provider of data. Mobilizing a sufficient number of participants will call for a society-wide campaign. It will be successful only if one can provide users with simple-to-use tools and guarantees of privacy, efficiency and reliability of the processing. This is a key reason for limiting the type of data they will have to provide to access to works or making them available (and possibly recommending and referencing them). For the sake of realism, we have assumed that when the Creative Contribution is first implemented, given suitable preparation, one could obtain a sample of $\mathrm{I} / 400^{\text {th }}$ of broadband Internet users in a given country, i.e. 400,000 in the US and 100,000 in France.

The key issue is how participants will provide use clues. The process we propose is as follows:

- Any individual can register to become a member of the sample. They will have to provide personal details, as well as a valid email address, under guarantees of privacy. Cryptographic keys permitting authentication of the reporting of use clues will be generated on the user's machine (for their use, see appendix C.4). Users will be advised to keep identifier and keys secret. Despite our desire to make participation in the sample as easy as possible, it might be necessary, as stated above, to slow this registration step by requiring some handshaking or other methods preventing abusive registration, for instance by robots.

- Participants will then be encouraged to choose from a number of free/open source software plug-ins to various applications involving contents access and upload: Web browsers, ftp or news clients, $\mathrm{P}_{2} \mathrm{P}$ clients, blog and other publishing software. ${ }^{15}$ They will be able to configure this software to abstain from reporting clues, depending on the type of use or contents, as far as the latter can be unambiguously identified.

- Use clues will be accumulated on the user machine and transmitted to the data collection agency when the user decides, at least once a year. Before this transmission, the user will be in a position to review their contents and delete any information s/he does not want to transmit. The mode of transmission of information to the data collection agency will limit the possibility to create fake members of the sample. Additional measures rendering the reporting of fake clues more complex or easier to detect are outlined in appendix C.

- Optionally, it will be possible to prevent transmission of use clues of a work when the participant is a potential recipient of rewards for this work. Of course, nothing will prevent creators from asking their friends to register and 
accumulate use clues for their works, as this is not in itself fraud, but it will be a time-consuming way to obtain rewards.

Data provided by content sites (user-generated content sites, voluntary sharing communities) and intermediaries will be of a similar nature: it will consist of anonymous information on access, upload and possibly recommendation and reference (anonymous with regard to users, of course). These sites will incur costs for providing it, and it would be normal for them to receive compensation payments from the data collection agency. They could be offered a guarantee of confidentiality: all consolidated data will be public, but which data elements originate from a particular site could be kept confidential. Content sites should not be obliged to provide use clues, but they can be encouraged to do so, as it contributes to rewarding contributors, from which they benefit. It is difficult to know how the balance between direct inter-individual sharing and access/upload through sites will evolve in a context of legalized sharing. However, it is likely that content site data will initially be at least an order of magnitude greater in the number of clues it provides than data from the voluntary user sample (see below).

The observation of Internet traffic would be an inefficient and fraud-prone mechanism if it were used directly for rewarding. It can nonetheless prove very useful to cross-check the received clues against some reasonably independent data source, in order to detect potential fraudulent reporting. Data from intermediaries such as Flattr or companies providing sampled data as Noank Media planned to do could also be useful complements. ${ }^{16}$

\subsection{Performance in one medium}

If rewards are to be distributed according to the relative use of works within each medium, we have to estimate to which degree of precision we can measure this use. In particular, are we able to measure use well enough for moderately popular works, whose authors intend and appear to deserve to be rewarded? Appendix C describes a model for the study of this question. We give the results of applying the model to two quite different medium/country situations: music rewards in the US and personal blogs in France. In both cases, our modeling shows that it would be initially really challenging to achieve the required level of precision and to disincentivise fraud, if one used only a voluntary sample. However, it becomes tractable if one also uses selected data provided by content sites.

We first present the results of applying our models to the voluntary sample of Internet users, and then discuss how the combined use of content site data enables some of the limits of a voluntary sample approach (when this sample is not sufficiently exhaustive) to be overcome.

The key parameters in the model are: 
- How much non-market use of works is likely to occur on average per person or per work, and as a result how many clues will be collected from the average participant in the measurement sample or from content sites?

- The model makes an assumption about how many creators would deserve to be rewarded in the medium under consideration, without assuming that each work will be necessarily registered for rewards from the Creative Contribution.

- Finally, how diverse will the distribution of popularity of works be?

Our estimates of these parameters are not necessarily accurate, but when in doubt, we assume the most unfavorable values, that is the values that will lead to a less precise measurement, or we signal the imprecision of the estimate.

\section{Music singles in US}

As detailed in appendix C, we worked on the hypothesis that, in the US, a fourth of all rewards would go to music creators. This means that, initially, 210,000 music artists would need to be rewarded at a level of $\$ 200$ or more, and $\mathrm{I}, 050,000$ at the level of $\$ 40$ or more. When considering other parameters, such as the average number of music tracks per creator and the proportion of titles registered for rewards, this would imply measuring the use of 3,780,000 music titles with sufficient precision.

Based on these hypotheses, if file sharing reporting by members of the voluntary sample would be the only source of data, one would obtain between 333 and 536 use clues per year for a creator rewarded at the "minimum significant level" of $\$ 200$. The number of clues for a creator rewarded at the minimum level of $\$ 40$ would be between 66 and 107. These figures were obtained using independent techniques (see appendix $\mathrm{C}$ ) and the difference in results is an indication of the difficulty of obtaining a precise estimate. Even with the worst estimate, the precision of the measurement coming from the voluntary sample would be correct: for a reward of $\$ 1000$, corresponding to $\mathrm{I}, 665$ use clues, the measurement error would be less than $5 \%$ more than $95 \%$ of the time. ${ }^{17}$

However, injecting a fake clue in the data collected from the voluntary sample would generate an artificial benefit (in terms of rewards) of $60 \$$ (37\% in the best estimate), making fraud attractive. This means that the parallel use of content sites data (download or streaming from user-content generated sites) is a necessary complement to the measurement system for a medium such as music in a very large country. It is not easy to estimate precisely how much additional data it would provide, but a conservative estimate is that it would add at least 5 times more clues per work. 


\section{Blogs in France}

As detailed in appendix C, we worked on the hypothesis that $5 \%$ of all rewards would go to bloggers. This is a relatively high proportion for a new Internet medium, which is only one among many, but it is justified by the current enthusiasm for blogging in France. For a population of 64 million, there are 9 million French blogs accessible on the Web, of which about 2.5 million are active and at least 500,000 are active and exhibit a creative effort. ${ }^{18}$ The number of active blogs is decreasing as some are being replaced by new social media such as Facebook, but the number of "creative effort" blogs keeps increasing.

Based on this hypothesis of $5 \%$ of rewards for bloggers, 57,500 bloggers would have to be rewarded. Considering that the registration of blogs for rewards is likely to be partial, usage needs to be precisely measured for twice that number, that is II5,000 blogs.

Based on an additional conservative hypothesis of the average yearly readership for these blogs (20,000 visits per year), assuming a distribution of attention more diverse than the general one for all media, our model predicts the initial voluntary panel would generate II8 use clues for a creator at the "minimum significant reward" level, and 33 for a creator at the "minimum reward" level.

This means that the data coming from the voluntary panel alone would be insufficient for a precise usage measurement. In addition, the risk of fraud would be high: injecting a fake clue in the reward system would be worth €I.27.

For such situations, the parallel use of content sites or blog software such as WordPress for the automatic reporting of use clues is even more necessary, at least until a much larger voluntary user sample can be put in place. It would be immediately efficient as it is likely that one could obtain reporting for at least a fourth of all significant blog access, thus obtaining 50 times more data ${ }^{\text {I9 }}$ than from the voluntary sample. This would meet the necessary demands for precision of measurement and disincentivisation of fraud. However, we advise against using only content site data for two reasons: data provided by sites is not to be trusted more blindly than user-reported data, and the use of a voluntary sample manifests the contributive nature of the overall reward system.

\subsection{Management costs}

Any large-scale reward system will necessarily have management costs. These should be in the range of a few percent of the distributed rewards. A non-market sharing reward system, thanks to its extensive use of the Internet, will have lower management costs than existing collecting societies. Nonetheless, maintaining the registers, collecting data on a large scale, detecting and preventing fraud, interfacing with financial management organizations and overseeing their activity, and conducting periodic surveys on Internet use will require significant bud- 
gets and highly competent staff. For comparison, the US Copyright Office budget is more than $\$ 50$ million, of which a large share is funded by user fees.

We expect that a management cost of $2 \%$ of the total amount of the Creative Contribution will be necessary for the operation of the agency or agencies described in chapter 9. On top of this, there will be costs associated with the financial management of the distribution of rewards and funds, which will probably represent a similar amount. A total percentage of $4 \%$ (\$2I4 million for the US, $€ 35$ million for France and $€ 49$ million for Germany) would remain well below the management costs of existing reward and incentive systems, including those managed by private organizations: Kickstarter, for instance, keeps $5 \%$ of the collected funds for its management costs and profits. ${ }^{20}$ 


\section{Clarification and counter-arguments}

In recent years, a lively debate has developed in countries where proposals involving a flat-rate contribution of Internet users to creative activity were made with a view to acknowledge or legalize file sharing. Most of these proposals differ from the one developed in this book because they adopted a compensatory vision of the contribution. In this chapter, we consider some of the most common criticisms. Some of them we took into account when designing our own proposal, so that they do not apply to it.

We attempt to provide short and clear answers to key points or questions, in the spirit of a "frequently asked questions" (FAQ) document. The "questions" are split into three categories: requests for clarification, counter-arguments raised by defenders of the right to share on the one hand, and by opponents on the other hand. This exercise is not by any means intended to close a debate, which we very much welcome, but rather to make our position known, so that the debate can proceed on a clear basis.

\subsection{Clarification}

1. Which media are covered by the Creative Contribution?

All media covered by copyright or author rights except software and copyrightable databases. The case of scientific publications is undecided. Note that this definition aims at ensuring that the rewards or support are granted only to original work. It should not be interpreted as implying that the copyright is the basis for the rewards.

2. What about non-copyrightable material such as data, facts, news and other material that is in the public domain?

Nobody can claim rewards for public domain material. However, projects whose aim is to make the public domain more accessible, and/or freely reusable, are legitimate recipients of the Creative Contribution, as they are part of the environment of creation.

3. Why are software, copyrightable databases and possibly scientific publications not included? 
Because other solutions are already established or being explored in these domains. ${ }^{\mathrm{I}}$

Our first duty in these domains is to abstain from creating further obstacles to the free access to published knowledge. In this regard, the European Directive on the Legal Protection of Databases ${ }^{2}$ must be repealed. Then, in some cases, new financing models are needed to permit some types of software innovation to take place in the commons or to make useful editorial functions sustainable in scientific publishing. Specific approaches are already being explored in these domains. Finally, competition policy to prevent discrimination against free/open source software is also important.

We chose to limit the scope of the Creative Contribution to informative, public expression or creative works because it seemed to us that the existing approaches might be disrupted if it encompassed software, databases and scientific publications.

If this position is reconsidered, it is possible, in principle, to include software and copyrightable databases in the Creative Contribution, but the global amount of the contribution and the definition of what is a work would need to be adapted. Specific work would also be needed on usage measures.

Finally, the exclusion of scientific publications may create difficulties, as they are not necessarily easy to distinguish from other types of publications. This may call for further debate, as their inclusion in the Creative Contribution would not necessitate an adaptation to our proposal. The decision may depend on whether one considers scientific publications to be read principally by the scientific community or by a wider public.

\section{Which works are covered?}

Any work in any medium included in the scheme, which has been distributed or made accessible in digital form to individual users, free-of-charge or against payment.

5. Why is digitizing of analog works or performances not included?

The world of freely sharable digital works has great promise and has already empowered each of us, but analog distribution remains of value, and the two will likely co-exist for some time. Expanding on Marco Ricolfi's copyright 2.0 idea (Ricolfi 2009), we propose to treat them as separate, in order to facilitate the transition. This amounts to maintaining one critical form of media chronology, separating analog distribution or performance from digital distribution. One can also describe it as recognizing an exclusive divulgation right for the author to authorize digital distribution, which the freedom of nonmarket sharing does not override.

It means that one will not be able to use the right to share to digitize and distribute orphan works or "out of print" works. As these represent a very 
important share of our culture, other means of recovering this heritage must be explored. For orphan works, one possibility is collective licensing, without compensation, but with a guarantee fund set aside to compensate any right holders who may re-appear, and thus to provide legal security for users. For "out of print" works, decent publishing contracts already include a clause, which returns all rights to the author if the publisher refuses to re-publish their works on a given (and brief) time scale, and this clause could be made compulsory.

\section{Is modification of works permitted?}

We consider that an additional debate is necessary to decide if non-commercial "remix" rights should be included or only the modifications that are permitted in Creative Commons No Derivative Works licenses: "such modifications as are technically necessary to exercise the rights in other media and formats". ${ }^{3}$ The arguments for each of the options are detailed on page 85 . If the choice is made of authorizing modifications, some adaptation is needed in the reward system. The simplicity of the reward system should be preserved, meaning that derivative works that meet originality standards are eligible for rewards in their own standing. This means that registering a derivative work is a statement of originality and can be challenged by the original authors. Of course, if authorized, modifications must be properly labeled.

\section{How is the total amount of rewards split for various media?}

A debate must be held to decide if the usage data will be collected and rewards distributed medium per medium, or if all can be managed in one basket. Our view is that a medium per medium approach is initially more credible. Our all-media-encompassing analysis in chapter 7 is useful for estimating the global amount of rewards needed. However, the definition of a work and usage patterns differ so much across different media that we consider it difficult, at least for the time being, to distribute rewards by mixing works of all media in one basket. This means that the global amount of rewards will have to be split into shares for various media. Of course, one would like to have an objective basis for doing so, but as we remarked in chapter 7 , there is no clear ground truth basis for this split. Neither the total amount of information shared on-line (in bits) nor the time devoted to usage of works in each medium are reasonable choices. Using the existing share of each medium in the present economy does not make much more sense.

One possible approach would be to use Internet user preferences expressed on a yearly basis (each person would allocate so many percent for written works, so many for music and other sound recordings, so many for video, etc.). However, some degree of certainty regarding the sums likely to be allocated to each medium is highly desirable. We have thus proposed that the 
distribution among media should be decided by a policy governance mechanism outlined in chapter 9 .

8. What is the main scheme for measuring usage in each medium?

We propose to obtain usage data from a voluntary subset of Internet users. When some components of the media industry attempt to enforce the monitoring of unauthorized acts by members of the public, they naturally encounter fierce resistance. As a result, it is hard for the industry to imagine that the public might be willing to cooperate in any form of usage measurement. However, a fair and efficient distribution of the collected sums is in the interest of Internet users, who are likely to be the best allies in this task.

Of course, there will be attempts to obtain abusive rewards, either through fraud or through efforts to bias measurements. Chapter io details a system that is reasonably robust, aiming at making fraud detectable or not the worth the effort. This system uses additional sources of information to complement the data provided by the sample of voluntary users.

9. Who decides who gets support for production projects?

The subscribers to broadband Internet who pay the Creative Contribution will allocate support to production. To make this choice easier and more efficient, we propose using a competitive intermediary model, where users allocate funds to intermediaries that compete to attract them, based on their policies or projects. These intermediaries can be participative financing organizations, participative production firms, including ones for large individual projects, or even public funds for the arts, culture and media, when their statute authorizes them to receive external funds from individuals. The choice would be made on a yearly basis. It may be advisable to limit the maximum percentage that a given user may allocate to a single organization, as suggested by (Muguet 2008). To be included in the list of possible recipients, intermediaries will need to satisfy some transparency and democratic governance conditions. Of course, compliance with these conditions will need to be monitored, and Internet users can also contribute to this effort.

\subsection{Criticisms by opponents}

10. Non-market sharing is a black hole, which will swallow the creative economy whole.

Many critics voice this fear, but it is not supported by presently available evidence. Today's digital media situation is characterized by: 
- a significant level of voluntary sharing by authors of their own productions for short texts, photographs, new multimedia and video formats, and music for some genres (techno, electro, for instance),

- a significant, and constantly growing, level of unauthorized sharing, by various means, of digital works in all media.

There is no evidence of a shrinking of the overall creative economy. However, it would be obtuse to deny that information technology and the Internet have introduced a radical change: the public is becoming a distributor of works in its own right. Some economic actors, whose traditional business models rely on having exclusive control over copying and distribution, need to partly rethink their modus operandi. This is particularly true for phonorecord publishers, and to a lesser extent for video publishers. It is important to realize that their activities represent only a relatively small share of the economy of the relevant media, and will only be affected partially. However, the role of these publishers goes beyond mere reproduction and distribution, and it would be a serious cultural loss if some of their other roles (such as editorial selection, support and promotion of artists) were to disappear. Some aspects of our Creative Contribution proposal are intended to help the re-invention of these roles in the new context. Nonetheless, the corresponding economic players will have to adapt significantly. Until now, some, in particular major phonorecord publishers, have clung to the fiction of retaining exclusive control of copies. The result has been a disastrous reduction of the volume of the market, while new economic players offered a growing variety of titles, but were starved of access to distribution and promotion channels. The recognition of a right to share will break this vicious circle, particularly if it is associated with a stronger competition policy in neighboring markets, such as concerts and the distribution of phonorecords.

11. Why would people buy something when they can get it for free?

This is exactly what record publishers said when music became an important part of radio broadcasting, in the Ig2os. At the time, records had only a weak competitive advantage over radio: they were not better quality, and certainly could not boast better ergonomics, when records had to be changed every three minutes. ${ }^{4}$ Their sole advantage resided in the possession of an item, and the ability to play it at a time of one's choosing. On the other hand, radio had its own competitive advantage, as a discovery channel for new music one did not know, or for live concert broadcasts. Radio soon became the key promotion channel for records. With time, this generated undesirable concentration effects, as radio stations were increasingly bribed or seduced into playing only a limited set of titles. ${ }^{5}$ But before that, radio turned out to be a blessing for record publishing. 
Today's situation is different, and people who sell digital contents will have to ensure their offer has a different value to users than what they can get from file sharing. This can take many forms, starting with a guaranteed level of quality, and the possession of the work in a particularly attractive form, or one that is easier to preserve in the long term than digital files. But commercial providers can also attract paying customers by helping them to locate contents of interest to them, by allowing them to communicate with other users with similar interests or to interact with artists, or by providing extra functionality (for instance synchronization with scores, or navigation functionality in a motion picture) or other forms of added value bonuses. Even without such added value, selling digital contents that are also accessible for free can work, in particular when you allow people to do what they want with them (in the non-commercial realm). Of course, the prices will have to be reasonable.

12. This will prevent us from reaching a commercial Eldorado.

Let us put ourselves in the mindset of an imaginary content industry entrepreneur. "What if we could sell contents at monopoly prices, and charge more for more desirable items. We could prevent people from copying works, and even make them pay for each usage. And all this with reduced costs of production and distribution: we would get immensely rich. This perspective is more attractive than the gold rush."

Unfortunately, this Eldorado is unattractive for the rest of us. To reach it, the entrepreneur in question will have to trample on just about every human right and every freedom necessary to exercise those human rights: the right to privacy, the freedom of information and communication, the right to due process and a fair trial, the right to access to education and knowledge, and the freedom to use the technologies of one's choice. ${ }^{6}$ The society needed for the Eldorado of on-line content sellers to exist would bear a striking resemblance to the worst dictatorship we know of, as far as our freedom to use the Internet and information technology is concerned (although the motivations for surveillance, control and repression would be different).

Of course, most of the players who are searching for commercial success do not want to do so at such an extreme cost. It was a refreshing experience when during the debates on the "three-strikes and you are out of the Internet" HADOPI law in France, some key figures from the French movie scene (producers, film-makers and actors) stood up and declared that they were not ready to stage a war against their public (Lettre aux spectateurs citoyens 2009). However, even a few more steps in the direction of this fabled Eldorado could already cause irreparable damage. It is not unrealistic to imagine that a dual society would arise, where less-informed consumers would be imprisoned in the Eldorado, while a small niche of free-thinking citizens 
would enjoy the benefits of information and knowledge sharing, albeit in a degraded form and at no small legal risk.

This is certainly not what content industry entrepreneurs want: they want to earn money by serving the public, the artists and the quality of their productions. We sincerely hope that their know-how will be used to make our imperfect real world a little better.

13. Why do we need file sharing when we already have streaming and centralized download sites?

Benjamin Bayart, the president of an associative Internet Service Provider in France, answered this question (Fradin 2010, excerpt from our translation):

Some obvious conclusions from the analysis presented in the report [of the French government on Network neutrality] were not drawn by the report itself. For instance, it explains that traffic has moved from a decentralized $\mathrm{P}_{2} \mathrm{P}$ model, which does not lead to international imbalances, to centralized traffic on streaming platforms or Youtube-type sites. [The report] also mentions the [three-strikes] HADOPI [law]. However, it does not draw the obvious conclusion. Why is the share of $\mathrm{P}_{2} \mathrm{P}$ decreasing? Because it is being attacked from all sides. It is the healthiest technology for a stable, strong and sustainable network growth without problems, and it is this precise technology that [existing policy] is trying to stop. $\mathrm{P}_{2} \mathrm{P}$ distribution should be supported. It is the only [form of distribution] that will counter dominant positions such as Apple's Appstore. [If the current trend continues,] iTunes will in the end have a global monopoly on selling music. [...] Interpersonal exchange, directly from machine to machine, without technical intermediary, is the basis of the Internet. [...] The same contents can be distributed in less than ${ }_{5} 5$ seconds in $\mathrm{P}_{2} \mathrm{P}$ when it takes 20 to 30 minutes with a central server. $\mathrm{P}_{2} \mathrm{P}$ is currently the most efficient and rapid means of distributing contents without destabilizing or damaging the network, and it can exploit localization. For dogmatic, political and technically erroneous reasons, [some governments] have aimed at damaging the best functioning tool.

The principal effect of HADOPI is to displace BitTorrent downloads in favor of Megaupload or similar sites, i.e. to go from a smooth system which does not create problems in networks to a completely centralized system, which creates artificial congestion points that should not exist!

So yes, indeed, there has been a displacement from decentralized file sharing towards streaming and centralized downloads, but it is the product of a bad policy, and it is already creating problems that will no doubt serve as an excuse to implement worse policy (attacks against network neutrality). 
14. You are going to reward pornography.

There are several answers to this criticism, which will appeal to different people and possibly be followed in different countries:

- So what? The copyright system already does, and this was not held against it.

- For the same reason that copyright is less used by the pornographic economy than by other forms of contents, the share of rewards or support to pornography from the Creative Contribution will be less than the real share of its use. The main reason for this is that authors and producers of pornographic works will be less inclined to register within the system, because it might expose tax evasion practices or other shady aspects of their business. Another equally important reason will be that usage of pornography will be measured less completely, and fewer preferences for support to its production will be expressed, because users are likely to be reluctant to provide such data (even within a system that guarantees anonymity).

- Finally, countries can put in place policies that disavow rewards and support for the production of pornography. There are drawbacks to such policies, especially for funds which are not public money. They will be seen by some as hostile to freedom of expression and access to information. Others will point out that the definition of what is or is not pornography is imprecise. However, there are established policies which already implement such negative or positive discrimination in the support of cultural products. For instance, film and video collecting societies in countries such as Germany preferentially allocate production support to some types of contents (such as documentaries) and disfavor others. ${ }^{7}$

\subsection{Criticisms by defenders}

15. It is incompatible with innovative market offers.

On the contrary, we believe that there will be a strong synergy between the Creative Contribution and voluntary sharing platforms with associated commercial offers. These organizations currently operate in a hostile environment, in which the artists belonging to them draw no income from the platform's most important component: free usage of their works on the Internet. Creative Commons platforms such as Jamendo or Pragmazic struggle to attract more recognized musicians, because the artists using these platforms get only indirect benefits (benefits of notoriety for concerts and other activities, sales of licenses for commercial use). The fact that artists will also ben- 
efit, through the Creative Contribution rewards, from having their contents made available on the Internet, will remove this limitation.

Classical or collaborative news media players, which presently use a combination of subscriptions, donations, advertising income and, in some cases, public support, will have a new income channel, which will help them achieve sustainability. The many music or video self-production or co-production organizations which target omni-directional income channels (concerts, radio, TV, film, use in live performance or advertising, etc.) will also gain an additional source of income or support.

Some music labels such as the French Believe ${ }^{8}$ will face a more complex situation, as they presently target sales of digital contents almost exclusively. However, they can adapt their business to the new situation. Digital sales to end-users are compatible with the right to share, if they are properly positioned and complemented with an added value. Magnatune is in an intermediate situation: it already has a large share of non-commercially shared music, and develops licensing for commercial use, but it also makes digital sales to individual users.

16. It is incompatible with grassroots intermediaries and participative production.

Grassroots intermediaries and participative production organizations will benefit greatly from the Creative Contribution on the production side, drawing on an immensely extended base of potential contributors. They will also benefit indirectly from rewards, as artists will have an increased interest in involving themselves in participative endeavors mobilizing Internet users.

Fernando Anitelli's O Teatro Mágico music and dance group in Brazil is a living exploration of the synergy between sharing and participatory rewards. This Creative Commons label has sold more than 300,000 albums in Brazil and is also involved in Trama Virtual, an organization funded by companies to reward music shared on the Internet. Users send use or appreciation clues, and the money collected from the sponsors is distributed according to these user indications. Trama Virtual illustrates the difficulties experienced by voluntary participatory rewards in terms of scale: if the sums collected are too small in comparison to the number of groups that show up for rewards, the whole system distributes rewards only to a small number of artists or suffers from heavy transaction costs. With a Creative Contribution system in place, Trama Virtual would function at the required scale, but of course, this would mean that users contribute not just clues but also money, in addition to what they are already spending on buying albums and concert tickets. $O$ Teatro Mágico probably does not need it, but other groups in Trama Virtual do.

Organizations such as Flattr ${ }^{9}$ propose to put in place a form of bottom-up organized Creative Contribution among a voluntary community (of both artists and users). Flattr is play on words between flat-rate and flatter. It collects a 
monthly flat rate and redistributes it to the producers of works which are signed up to it, according to use data collected from members using a small software package (a simple button that users click to indicate appreciation of a work when accessing or using it). Will Flattr suffer because users will feel they have already paid for culture in general? In our opinion, this will not be the case, because Flattr has specific assets: it is intrinsically global in scope and can mobilize specific communities of interests, i.e. it can become a participative intermediary for specific groups. Finally, one would not trust the collection and distribution of sums on the scale of society to a private firm, but it would be possible for intermediaries such as Flattr to be providers of certified and auditable usage data for rewards. This would create an additional incentive for users to be members.

17. This is a new tax for the benefit of a few.

The Creative Contribution is not a tax, but rather a statutory payment. Many people see these two things as the same, but there is a key difference: the collected sums do not become part of the budget of a government. They are intended for one specific purpose and are managed by a specific organization. We have designed this organization to be under the control of the contributors: this is why we described it as a mutualized financing scheme.

Now, is this statutory payment for the benefit of a few? It is designed to be for the benefit of many in some ways, and the benefit of all in others. We could say that all will benefit, because all will have the right to non-market sharing, but that would not be fair: one does not buy a right, one just gets it. However, all will benefit from the richness of contents that can be shared on the Internet. Truly all: when the right to share is recognized, the existence of legitimate intermediaries will make sharing easy enough.

That is not the complete answer, of course. Most people supporting this objection worry that significant rewards would only go to a few. But what are we it comparing to? It may be a few, but it will be many more than those who currently receive significant rewards from copyright. This may not be enough for everyone's taste, but at least it is your taste that will decide who gets rewards. And finally, less-than-proportional reward functions can be used to tune the spread of the distribution of rewards.

18. The Creative Contribution will consolidate the poor governance of collecting societies.

Volker Grassmuck, one of the key proponents of the Kulturflatrate in Germany, was reported by (Cronin 2010) to have said: "'Mafia' is a widely used epithet for music collecting societies in many countries. The arcane area of collecting societies draws much public interest and anger." There is a key contradiction, inherent in our present situation: collective management is 
needed more than ever in the Internet age, because it is the only way to have an efficient, large-scale allocation of funds, without associating transactions costs to usage, but its present face is not attractive. The existing forms of collective management grew out of associations of co-opted authors defending their own interests. They were then developed by persons who were sincerely committed to culture and the arts, but have turned themselves into organizations that serve interests often quite remote from the full creative ecosystem. Publishers, heirs of right holders, investors who have managed to get copyright assigned to them, and a few best-selling artists control their governance, which is often characterized by censal voting. ${ }^{10}$ For these organizations, self-propagation and pursuing management interests are often higher on the agenda than the interests of culture as a whole.

We need to move away from this misgovernance, but we should not throw the baby out with the bath water. Our approach is to create brand new organizations, with a new governance basis, and to trust the key distribution functions only to them. They will handle the minimal criteria for the registration of works, the measurement of usage, the calculation of rewards for works, the collection of user preferences for support to production and the environment of creation, the organization of decision-making on how rewards are to be split between media, and the reporting to the public. The existing collecting societies will then have a choice. Either they must accept playing by the rules of a reformed system, amend their governance, accept caring for all creators and contributors and not just their members, as a few already have in various countries (SPEDIDAM and, for a period, ADAMI in France, and more timidly STIM in Sweden ${ }^{\mathrm{II}}$ ). Or they will continue to oppose these proposals, as most collecting societies have done, often to the point of supporting the most repressive approaches to file sharing. In this latter case, even the financial management of individual rewards will have to be conducted by a new organization.

19. In the name of commons, you are monetizing the non-market.

No, we aren't, but the difference is a subtle one. We are financing the conditions of existence of a specific form of cultural commons. In a world where access to conditions of living and resources for production are monetized, commons can exist only if those who maintain and enrich them have adequate financial resources. The key differences between monetizing the nonmarket as described by Jeremy Rifkin in the Age of Access (Rifkin 200I) and our proposal lies in:

- the absence of transaction and control in the path of usage,

- the empowerment of users. 
Social public goods and commons in modern societies are always financed by collective means. Publicly run schools are built by paid contractors, and teachers receive a salary. Elinor Ostrom, who received the 2009 Nobel Prize in Economics for her studies of commons governance, has stressed that the management of resources by user communities was a key approach to governing commons. The true question is "is our proposal the right approach to finance cultural commons in the Internet age?" We certainly did our best to define one of the ways of doing it.

20. This will not stop the war against sharing.

This criticism was expressed by anti-capitalist thinkers. They consider the war against sharing as the essence of capitalism and doubt it would stop just because of the recognition of a domain of non-market sharing of digital works between individuals. Our ambition is more modest. We just intend to make sure that some essential capabilities are not taken away from people, and we trust that they will exert them in an empowering manner.

21. This will undermine other sources of income for creators, such as salary and fees. This is a very legitimate concern. Each time a new form of delayed income is created, investors and employers may use it to put pressure on immediate wages and other forms of payment. This concern does not mean we should abstain, but vigilance is required. 


\section{From proposal to reality}

It is time to reflect on whether and how the proposals developed in this book can attract a critical mass of support. The last few years have seen a subtle but significant evolution. Proposals for a flat-rate financing of culture and the recognition of file sharing first arose as a reaction against repressive laws. Geographically, they followed the world-wide dissemination of these laws, which was co-ordinated by a few interest groups such as the International Chamber of Commerce or specialized media interest groups. ${ }^{\text {I }}$ Flat-rate proposals were made in response to DRM anti-circumvention laws, then to "three-strikes and you are out of the Internet" laws, and now to the present generation of laws requiring the compulsory filtering of sites. This process is still at work, but another agenda is developing: the financing of sharing-compatible digital creativity is seen as a goal in and of itself. This approach was present from the start, but less visible. In this concluding chapter, we outline the trajectories along which this autonomous agenda is unfolding. These paths will cross and part, blend and diversify, with unpredictable results. But they all have a role to play in laying the foundations for a sustainable digital cultural ecosystem, and making it a reality.

\subsection{Grassroots Internet and creative communities}

James Love's former Consumer Project on Technology and the present Knowledge Ecology International have been consistently promoting an independent vision of how culture could be financed and shared in the digital era ${ }^{2}$ since 2002. Several milestones were reached along the road. In 2006, the Paris Accord meeting brought together representatives of Internet policy, consumer groups and creative communities to try to define a common road-map on these issues. ${ }^{3}$ That same year, scholars and Internet policy specialists met in Chicago at the $10^{\text {th }}$ anniversary conference of the peer-reviewed on-line journal First Monday to reflect about how to make collaborative creativity sustainable. ${ }^{4}$ Even though these events received a significant amount of attention on the Internet, it is fair to say that the sound waves did not propagate far beyond circles specialized in the access to knowledge and culture.

The increasingly mingled grassroots Internet and creative communities have a key asset: they do not wait for things to happen, they make them happen. The ideas we presented in this book would have remained confined to small groups if content authors had not started to authorize sharing of their works. The voluntary 
sharing of information, expressive or creative works was formalized by licenses from 1998 onwards. ${ }^{5}$ This made explicit a practice which had in fact started much earlier: digital projects such as Project Gutenberg started close to 40 years ago, and the open Web as a whole can be seen as a giant voluntary sharing project, where "cut and paste" is the primary sharing tool. Enabling a self-conscious approach to voluntary sharing for any individual or group was a key step forward. Several hundred million works covered by copyright are today shared voluntarily, mostly under Creative Commons (CC) licenses. CC licenses organize the synergy between cultural commons and commercial activities, but they do not provide a way to finance the conditions of existence of the creative works and their producers. They are still used predominantly for works which can be produced by an individual with a minimal investment and some capabilities (Web texts, photographs, free encyclopedias such as Wikipedia and its satellite projects) or whose funding is ensured by a pre-existing public organization (scientific publications, for instance). There are also some remarkable voluntarily shared works in other media, which call for a stronger investment or require know-how that is less universal, but these remain a minority in their respective universes.

Treating a sustainable cultural commons as a self-standing goal, and in particular proposing that they should be financed via a flat-rate contribution by Internet users, can be seen as addressing these limits. Such proposals initially met a brick wall: not only media interest groups but also governments refused pointblank to consider them. There has been no government-initiated, official study of the costs, benefits, and more generally the impact of proposals such as ours. This has caused frustration in circles going well beyond the initiators of these proposals, in particular in countries where attempts to eradicate sharing have triggered a lively societal debate. Faced with this, Internet and creativity grassroots groups took matters into their own hands, and endeavored to set up co-operative financing systems along the lines of the entertainment co-operative proposed in (Fisher 2004, pp. 252-258). ${ }^{6}$ In the last 3 years, projects such as Kickstarter in the US and Yooook in France ${ }^{7}$ have flourished. Some of them even follow the flat-rate model, in particular Peter Sunde's Flattr. ${ }^{8}$ Flattr presents itself somewhat misleadingly as a social micro-payment platform, but micro-payments by users, the drawback of which were analyzed by (Shirky 2000), are precisely what it avoids. Each Flattr member pays a flat-rate contribution every month, which is shared according to the interest they manifest for the works of other Flattr members. In other words, Flattr is a social resource pooling and user appreciation-based distribution platform. In that sense, it is a true foreshadowing of our proposal's implementation, although there are important differences (Flattr has to track the appreciation expressed by each individual for each work). It is too early to know if Flattr, Kickstarter and their more local counterparts in many countries will scale up, and to which degree. If they do reach the scale needed to address global cultural commons challenges, they will be handling milliards of dollars per year. There will 
then be issues of governance for some of these intermediaries, as for any largescale money-handling organization.

Meanwhile, there is an excellent synergy between the concrete exploration of alternative financing systems and proposals for new policies for the society-wide pooling of resources under the umbrella of law. This synergy is not without tensions, just like there are possible tensions between voluntary sharing practices and the recognition of a certain level of sharing as a minimum right. Though the founders of Creative Commons, in particular Lawrence Lessig and James Boyle, are also copyright policy reformers who believe in installing a basic right to share, recognizing this right legally will, in a way, deprive voluntary sharers of the specific gesture of authorizing this minimal sharing. Similarly, some may see proposals such as the Creative Contribution as an unnecessary intrusion of policy in the set-up of resource pooling for the cultural commons.

Grassroots creative and Internet communities' representatives, academics and policy-makers now meet regularly in events such as the Free Culture Forum ${ }^{9}$ in Barcelona to discuss new models of sustainability for the digital era. The questions raised above are hot topics on the agenda. The outcome of the 2010 round of discussions is embodied in a How-To for Sustainable Creativity, which discusses I2 sharing-compatible mechanisms for providing resources to creative activity. ${ }^{\text {IO }}$ Flat-rate based resource pooling such as ours is but one of them, and the How-To lists important conditions for its acceptance by the very communities it is trying to empower. One thing is certain: a friendly and honest comparison between the various approaches is the best tool to improve them.

Our own vision is that, in addition to market mechanisms and public subsidies, it would be optimal to associate a general society-wide resource pooling set up by law with many competitive intermediaries ensuring focused financing or rewards in specific domains or geographical zones.

Beyond the aforementioned circles, the last ten years have seen the development of cultural craftsmanship on a giant scale. In the course of writing this book, we have encountered evidence for this at various stages: we mentioned that more book titles were published in the US recently on a "print on demand" basis than using the classical "print in advance" method, and described the proliferation of self-production or small production structures in music, and the related growth in the number of published albums. This modern and agile craftsmanship develops important editorial functions. Its practitioners are by nature fragmented, and it will take time for them to evolve a common strategy. Meanwhile, many are captured by bigger distribution interests, sometimes because they depend on the existing distribution channels, which the latter control, and sometimes because they are simply too busy to spend time building coalitions. Recognizing them as an important constituency and helping them define autonomous objectives is a meaningful policy agenda. We now turn to a key question: who will table proposals and vote to implement the Creative Contribution or a similar system? 


\subsection{Government policy}

We already mentioned that, up to now, governments have given little consideration to any form of flat-rate contribution to freely sharable cultural creativity, with the possible exception of Brazil, where there is some discussion of incorporating a cultural flat-rate within the on-going copyright reform. The draft law that was submitted to the public for comments in 2010 did not include a flat-rate proposal, though it is one of the most favorable for access to culture (Colombo 2010), but the issue is still under discussion. However, the nomination of a new Culture Minister has raised concerns about whether the original intentions of the reform will still be pursued (CulturaDigital 2010).

The reasons for this continued repressive attitude by governments increasingly go beyond file sharing itself: controlling the Internet as a sphere of public expression has become a major aim for some regimes, whether authoritarian or democratic.

An extreme example is Italy, where open access to the Internet, the free expression of bloggers, the protection of intermediaries against liability, the mere freedom of the media to report on judicial affairs, and the access to certain some sites, are all under threat or already hindered. Under the Pisanu decree of 2005, originally provisional but then turned into permanent Italian law, giving Internet access to anyone required obtaining a photocopy of a photo ID and retaining a memory of communications for the specific user (Masera 2010). This precluded easy cybercafe access or open wireless networks, including in universities, and Italians could envy the residents of many emerging countries in this respect. Though the Pisanu decree article regarding access to the Internet was finally reformed at the end of 2010, Internet access in Italy remains hindered by both legal and social obstacles. A law proposal plans to submit bloggers to the same rules as the press, even requiring video-bloggers to have a prior authorization of operation, as is the case for broadcasters (deMartin 2010). Another "gag law" proposal would prohibit, if adopted, reporting by any medium on the content of judicially ordered phone taps until a final judgment has been pronounced (DEramo 2010): the primary motivation seems to be the prevention of reporting on serious penal cases in which the Prime Minister and his advisers are involved. In both Europe and the US, Internet Service Providers and the hosters of content sites are exempt from liability for the contents they transmit or let users upload without checking them. This is called the "mere conduit" protection and was a key enabler for the development of Internet businesses and activity. However in a case against Google Video, an Italian judge issued a first instance judgment with jail sentences (presently under appeal) against Google executives, on a basis that seems to ignore the legal framework of European liability protection. ${ }^{\mathrm{II}} \mathrm{A}$ final judgment is still pending on whether the mandate issued by Italy to ISPs, ordering them to filter The Pirate Bay site as a whole, is legal or not. ${ }^{\mathrm{I2}}$ Only this last case concerns 
file sharing, but all are part of a general effort at installing preventive and preemptive control of expression on the Internet, its usage and its access.

This is of course worrying, but offensives against the open Internet and the freedom to use it without a priori control have unintended positive side effects. Many citizens and advocacy groups, which were traditionally remote from Internet issues, have now realized the extent to which freedom on the Internet has become an essential condition of democracy and an open society. Many who would before have accepted any measure presented as intended to fight piracy have now started to scrutinize such proposals more carefully. Some of them have even gone as far as to recognize that proponents of the legalization of file sharing care more about creativity than the proponents of the war on piracy. This is one of the factors which caused some major French motion picture figures to join the Création-Public-Internet coalition, which supports the Creative Contribution proposal. ${ }^{13}$

In itself, this is not enough to change governmental policy. However, it is helping to formulate a minimum set of demands, which can be pushed consistently in all democratic countries. One of these demands is that governments should commission independent impact studies and consultation processes on proposals such as the Creative Contribution. More generally, this kind of option should be considered in the relevant policy preparation exercises. These demands come not only from civil society and advocacy groups, but increasingly from parliaments and political parties.

\subsection{Policy-makers}

We already mentioned that a law amendment incorporating blanket licensing for file sharing was adopted in France in December 2005, before a new vote reversed the decision in 2006. An amendment putting in place a "creative contribution" for music was tabled twice by the opposition Socialist party in the 2009 parliamentary debates on the three-strike HADOPI law. Even after this law was adopted, as difficulties with its application were becoming apparent, a new law proposal for a blanket licensing for file sharing was tabled by a member of parliament belonging to the right-wing majority (Guillaume 2010). During the 2009 general elections in Germany, the Green and social-democrat parties included a Kulturflatrate proposal in their program. Without going so far as to endorse it, a spokesperson for the winning CDU party said the Kulturflatrate was an option to be included in policy debates. ${ }^{\text {I4 }}$ The recognition of file sharing and the financing of culture are issues that cut across the political spectrum, and it is interesting to make an inventory of how specific political schools of thought position themselves on these matters.

Green/ecologist parties have a natural sympathy for the recognition of commons and for the empowerment of individuals in creativity matters. Some of the 
most active supporters of the Kulturflatrate or the Creative Contribution in Europe are to be found among their members. However, the same reasons render them cautious about a possible adverse impact of schemes organized by law on grassroots initiatives. For them, the compatibility between the two is a key aspect of any proposal.

Social-democrats, or liberals in the US, are on familiar ground when matters of society-wide pooling of resources and distributive justice are at stake. However, in Europe, some of them fear that too strong an empowerment of individual preferences would endanger a more top-down, traditional cultural policy. They thus support public broadcasting more than broadcasting by the public. The attachment of social-democrats to socio-economic rights leads some to embrace cultural commons and their financing as an enabler of these rights, and others to consider the notion of non-market cultural commons with some suspicion because they fear that it could weaken economic growth. Recently, some have advocated the acknowledgment of the non-market commons contribution to empowerment and capability building as an essential strategy for renewing the social-democrat policy offer (LabideesPS 2010).

Liberals in the European sense (defenders of economic freedom and civil rights) are deeply divided: most reject a repressive approach to file sharing, but they are reluctant to involve government intervention in organizing another policy.

Some European conservatives or republicans (in France) disapprove of the influence of interest groups on policy-making in these domains and, as a result, favor government-organized rewards and the right to share. For many of their colleagues however, commitment to the general interest stops short of challenging the claims of large financial players. However, in times of deep economic crisis, these kinds of dividing lines become more fluid.

Parties coming from the communist tradition have great sympathy for the commons, but they sometimes share with Bill Gates a confusion between commonism and communism. ${ }^{15}$ They are inclined to support the direct provision of cultural services by the State, which makes them defenders of the digital accessibility of the cultural heritage but does not help them recognize the potential of a direct provision of access to culture by the citizens themselves.

Finally, many voters who consider themselves distant from all political parties and have a shifting voting record, as many active Internet contributors do, are influenced in their vote by Internet freedom issues.

\subsection{Entertainment players?}

Listing established entertainment players as one of the possible driving forces for the legalization of file sharing and the implementation of new financing models may be seen as paradoxical. Even analysts who showed a deep understanding for 
the way of thinking and the stated concerns of the entertainment industry were faced with its stubborn refusal to deviate from anti-piracy efforts. There are nonetheless reasons to think that some of the entertainment industry players will at some point embrace another vision. An indication of that possibility arose when, as we recalled earlier, Jim Griffin, then a strategist for Warner Music, made a proposal for a flat-rate licensing for file sharing for university students (Orlowski 2008). There are deeper reasons to believe that this is not the end of the story. The first one is that in the Read-Write Society that Lawrence Lessig advocates (Lessig 2006), there is room for Read-Only components. The continuum of positions which we described between producers and consumers, or between creators and receptors of works, also includes extreme positions: professional creators and producers, and consumers solely focused on accessing these works. Even the most prosumer-oriented Internet users enjoy laid-back entertainment when it is not forced upon them as the only cultural perspective. While not likely to be among the initial promoters, part of the established media industry can rally a proposal for new user rights and financial contributions if they see it is likely to become a reality. Their aim would be to conquer a share in the new sharing-compatible commercial offers.

The motion picture and video industry, while still pushing for DRM and other means of enforcing the scarcity of copies, could come to accept such a perspective, rather than engage in a permanent war with the public. The reason it is not doing so yet is that it is presently benefiting from the coexistence between unauthorized semi-clandestine sharing and oligopolistic control (on production in some countries, on key distribution channels in others). This is a cow that is worth milking for as long as possible. The US independent movie producers, who sadly followed RIAA in suing individual file sharers (See Anderson 2010), will in the end look at the potential contribution to production arising from our proposal.

As for the musical majors, they have milked the cow of scarcity to such a degree that it is dry, with so restricted an offer of new titles, that it can hardly be restricted further to maintain the extremely high per album profits margins they enjoy. When a new playing field seems established, they will move. When will this happen? We don't know. Who will take the lead? Most probably one of the less dominant players in the oligopoly.

\subsection{Collective management?}

For a while, it looked as if the collecting societies would be key supporters of a new flat-rate-based reward system. In France, the collecting societies for music performers (SPEDIDAM and ADAMI) were part of the Alliance Public-Artistes that promoted the 2005 blanket licensing proposal. ${ }^{16}$ In Sweden and Canada, the music collecting societies also made similar proposals. In Italy, SIAE showed 
some interest in discussing it at least. The involvement of collecting societies raised concerns about governance, equitable distribution including for non-members, and adaptation to Internet conditions. But as they also brought a significant know-how in managing large-scale financial distribution, and as they carried a significant political momentum, their participation was seen as a welcome rupture from the repressive agenda.

Unless they can show a determined change of position and attitude soon, it is now probable that they have missed the window of opportunity: few believe they will come aboard any reasonable scheme. During the past few years, collecting societies have been among the most active promoters of new control, surveillance and repression measures, going as far as tabling the amendments that were most harmful to Net Neutrality and Internet freedoms during the revision of the European telecommunication regulation (Quadrature 2008b). Their stubborn refusal, in almost all countries, to accept management of commercial rights for works licensed under Creative Commons Non-Commercial licenses, infuriated even the most moderate. They did not move an inch on issues of governance, and they won't until governments adopt provisions such as those proposed in the Brazilian copyright law, for example, which states (Colombo 2010):

(iii) the entities entitled to revenues and distribution of rights shall reduce administrative costs and deadlines for distribution of the amount to the rights holders, publicize all the acts of the institution (more transparency), particularly the collection and distribution.

This seems to leave little chance for the collecting societies to act as the "financial management organizations" we described in chapter 9 in any implementation of our proposal, and it seems even less likely that they will be counted among supporters of this kind of proposal in the coming debates. Nonetheless, once hundreds or thousands million dollars are to be distributed, their members, and even their management, will no doubt have second thoughts. When this happens, the promoters of flat-rate proposals will be in a position of strength, and should put all their requests on the table. There are key elements of the social distributive justice which we stand for, that we simply cannot trust the collecting societies with in their present form: the collection of use and preference data, the computation of rewards and the governance of multi-stakeholder decisions. They can nonetheless contribute significantly to a smooth distribution of rewards and facilitate the registration of works, provided that they understand that this a new role, not their private turf. 


\subsection{The continued role of academic research}

Academic research played a key role in challenging the accepted wisdom on the impact of file sharing and in elaborating proposals for new financing schemes for creativity on the Internet. In times to come, it will no doubt keep playing this role, but it will have to do it in a complex context. As we near the possible implementation of proposals such as ours, research and advocacy increasingly mingle. The Free Culture Research workshops and conferences ${ }^{17}$ are a meeting ground for their interaction, an interaction that also takes place within each of us.

Researchers have a civic role, and they must, more than ever, involve themselves in societal matters and debates. But this civic role includes reminding everyone of the complexity of the matters we are trying to address. Reducing them to a few abstract principles of property or maximizing profits fits some agendas. Adopting a simple "just-do-it" approach to complex matters would not serve the public interest any better, and neither would it advance knowledge. 

Appendixes 



\section{A Diversity of attention for beginners}

Throughout the book, we analyzed the diversity of attention to works, as well as of the distribution of revenues, which those who produce them receive from various channels. Sometimes we have exhaustive data to study the diversity of attention directly, but in many cases we have only some partial or indirect evidence or hints about what its future may look like in various conditions. The whole debate on the Long Tail theory formulated by Chris Anderson (Anderson 2004, Anderson 2006) revolves around the way in which the diversity of attention is likely to evolve in various situations (see below section 13.4). We need then to model the diversity of attention, and for this purpose we used a mathematical model known as Zipf's law. This appendix is intended to give the interested reader a brief introduction to the modeling of diversity of attention in general, and to explain why Zipf's law is used, how it works and its limitations. Less mathematically inclined readers should not be deterred by the presence of equations: one can ignore them and still understand the text. However, the equations will be helpful for those who wish to verify the mathematical reasoning for themselves, or to carry out further investigations.

This appendix uses ideas from the ranking tutorial of Lada M. Adamic (Adamic 2002b) and from the remarkable Wikipedia page on Zipf's law. ${ }^{\mathrm{I}}$

\section{A.1 From wealth to popularity}

\section{Wealth distribution}

Librarians have probably studied the relative demand for books or other texts for centuries. Even as far back as the Middle Ages, it was a key factor for organizing the production, purchase and storage of copies of books and other texts. We may thus speculate that ancient librarians compiled lists of books (or other items) sorted according to demand, with the most frequently requested items first, and some of these registers may yet survive in library archives. If so, they would be the first evidence of popularity distribution studies. However, it seems that a mathematical modeling of similar distributions emerged in other fields. At the end of the Igth century, the Italian economist Vilfredo Pareto formulated what is now called Pareto's law, according to which the number of people who have more 


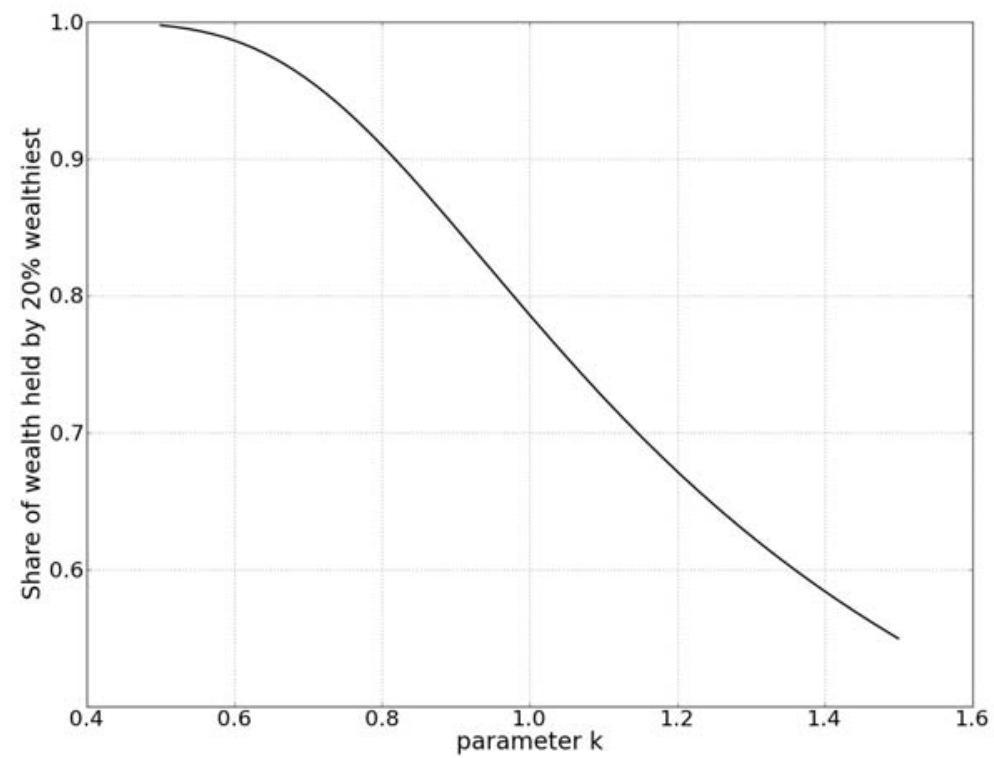

Fig. A.1. Share of total wealth held by the $20 \%$ richest individuals, depending on the value of Pareto's law parameter. If the wealth distribution in a particular group is described by a Pareto's law with parameter $\mathrm{k}$, this graph shows the share of the total wealth held by the $20 \%$ richest individuals, depending on the value of $\mathrm{k}$.

wealth (or own more land, or have greater income) than a given number $\mathrm{x}$ is inversely proportional to $\mathrm{x}$ raised to a characteristic constant power $\mathrm{k}$. The corresponding probability distribution can be formulated as:

$$
\mathrm{P}[\mathrm{X}>\mathrm{x}] \propto\left[\frac{\mathrm{m}}{\mathrm{x}}\right]^{\mathrm{k}}
$$

where $\mathrm{x}$ is the wealth of the person under consideration, and $\mathrm{m}$ is the wealth of the poorest person. Throughout this appendix, we use the symbol P followed by square brackets to mean "the probability that [the expression in the brackets] is true", and the symbol $\propto$ means "directly proportional to". When k is just below I, about $20 \%$ of the people own $80 \%$ of the wealth, which has become known as the Pareto principle. As often happens, this rule of thumb has become well-known, but the law is less known, and people tend to forget that it has a parameter. Figure A.I shows that when $\mathrm{k}$ is varied from 0.5 to $\mathrm{I} .5$, the share of wealth held by the $20 \%$ richest individuals can range from $99 \%$ to $55 \%$. The full Pareto principle actually states two things: that the distribution of wealth follows Pareto's law, and that, in some observed situations, its parameter is close to I. 
Pareto's law describes a family of functions which are examples of the wider class of power law distributions. These are so called because their probability density functions ${ }^{2}$ follow power laws, meaning that it is proportional to a negative exponential power of the variable. In the case of Pareto's law, the probability density function is:

$$
f(x)=\frac{k m^{k}}{x^{k+1}}
$$

Pareto's law is thus a power law distribution with index $\mathrm{k}+\mathrm{I}$. Power law distributions are extremely widespread in nature, where they arise from processes which have no preferred scale. They also crop up often, and most relevantly for this book, in the study of ranked distributions, namely distributions of values that have been ordered by decreasing value.

\section{The star of the show}

For convenience, we would like to characterize the degree of diversity of attention to cultural works with a single number. The best way to do this is to model the ranked distribution of the number of times each work has been accessed using Zipf's law, which has been shown often to approximate closely the real-life ranked distribution of popularity of cultural works.

George Kingsley Zipf was a Harvard University linguist who studied the frequency of occurrence of words in different languages. He formulated the law that bears his name in 1935 , though the same pattern was already noticed much earlier, probably as early as 1916, by a French stenographer named Jean-Baptiste Estoup (Petruszewycz 1973). Estoup wrote a stenography manual which was reedited many times, and he used his analysis of the frequency of words to design his method, but he did not model it as systematically as Zipf.

What Zipf stated is that, if one ranks the number of occurrences of words in a large text, starting with the most frequent word and continuing in decreasing order of frequency, then the number of occurrences $\mathrm{O}$ of each word is inversely proportional to its rank k elevated to a constant power $\alpha$ :

$$
\mathrm{O}(\mathrm{k}) \propto \frac{\mathrm{I}}{\mathrm{k}^{\alpha}}
$$

Zipf also remarked that the parameter $\alpha$ was close to I, so that the $100^{\text {th }}$ most frequent word is approximately roo times rarer than the most frequent word. As with Pareto's law, this rule of thumb has stuck, and commentators often forget that the parameter can take other values. 


\begin{tabular}{|c|c|c|c|}
\hline & $\begin{array}{l}\text { mbre } \\
\text { les } \\
\text { titions }\end{array}$ & & $\begin{array}{l}\text { Nombre } \\
\text { des } \\
\text { répétitions }\end{array}$ \\
\hline 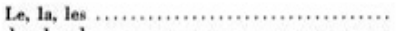 & 1949 & 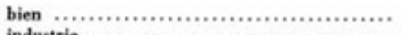 & 53 \\
\hline 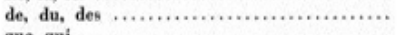 & 1712 & industrie ................................ & 50 \\
\hline 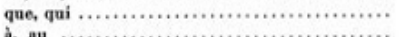 & 766 & 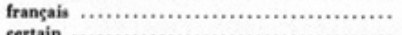 & 48 \\
\hline 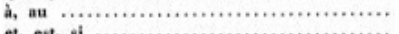 & 743 & 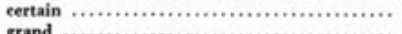 & 44 \\
\hline 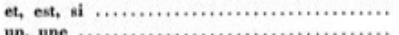 & 741 & 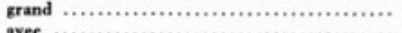 & 44 \\
\hline 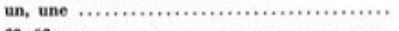 & 413 & 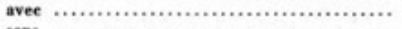 & 41 \\
\hline 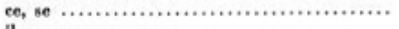 & 393 & 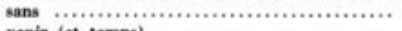 & 40 \\
\hline 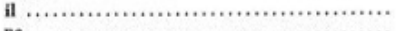 & 282 & 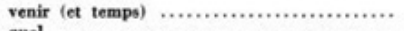 & 39 \\
\hline 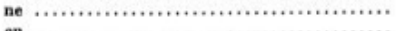 & 279 & 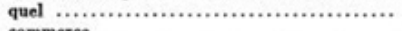 & 39 \\
\hline 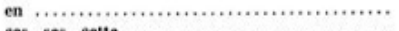 & 258 & 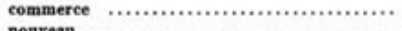 & 38 \\
\hline 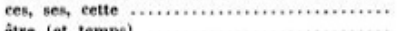 & 232 & 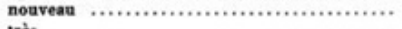 & 37 \\
\hline 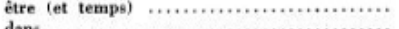 & 200 & très & 37 \\
\hline 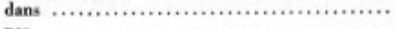 & 181 & 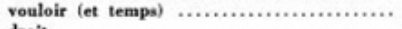 & 37 \\
\hline 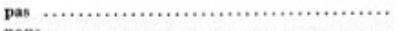 & 179 & 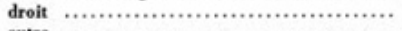 & 36 \\
\hline 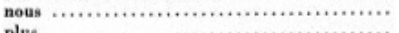 & 167 & 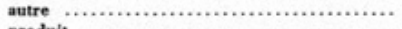 & 36 \\
\hline 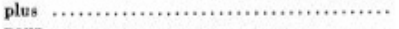 & 162 & 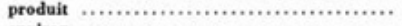 & 36 \\
\hline 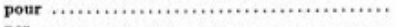 & 157 & 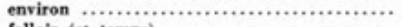 & 35 \\
\hline 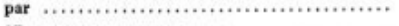 & 155 & 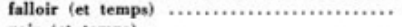 & 35 \\
\hline 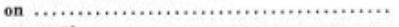 & 155 & 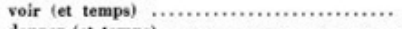 & 35 \\
\hline 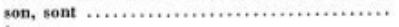 & 141 & 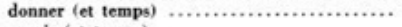 & 34 \\
\hline 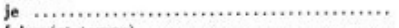 & 137 & 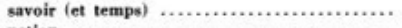 & 33 \\
\hline 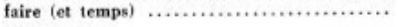 & 136 & 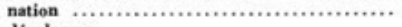 & 32 \\
\hline 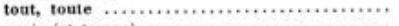 & 119 & 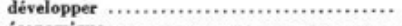 & 31 \\
\hline 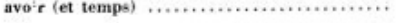 & 118 & 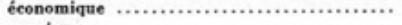 & 31 \\
\hline 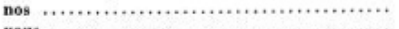 & 115 & 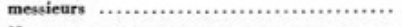 & 30 \\
\hline 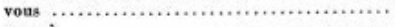 & 108 & 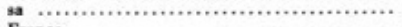 & 30 \\
\hline 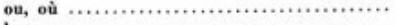 & 100 & 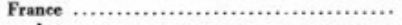 & 29 \\
\hline 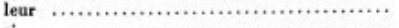 & 94 & 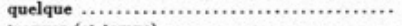 & 29 \\
\hline 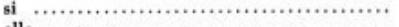 & 93 & 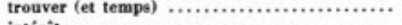 & 29 \\
\hline 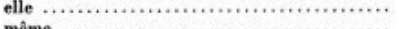 & 92 & 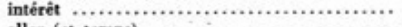 & 29 \\
\hline 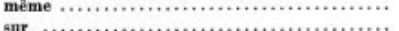 & 84 & 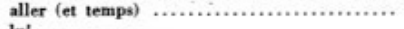 & 28 \\
\hline 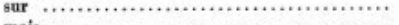 & 82 & 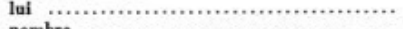 & 27 \\
\hline 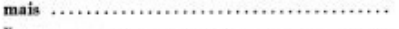 & 82 & 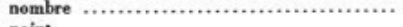 & 27 \\
\hline 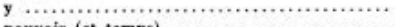 & 70 & 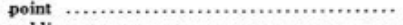 & 26 \\
\hline 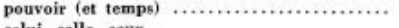 & 66 & 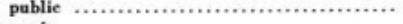 & 26 \\
\hline 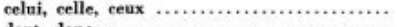 & 62 & 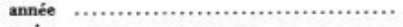 & 25 \\
\hline 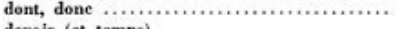 & 62 & 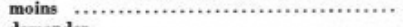 & 25 \\
\hline 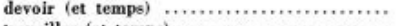 & 60 & 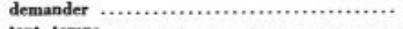 & 25 \\
\hline 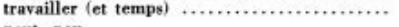 & 60 & 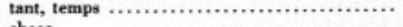 & 24 \\
\hline 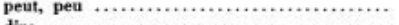 & 58 & chose ................................................ & 23 \\
\hline dire & 57 & 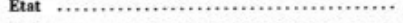 & 23 \\
\hline & 56 & & 23 \\
\hline
\end{tabular}

Fig. A.2. The number of occurrences of words in a French text of 30,000 words, as reported by Estoup, reproduced in (Petruszewycz 1973).

The use of "Zipf's law with parameter close to one" has become part of the folklore in several fields: linguistics and bibliometrics, of course, but also more recently Internet studies (Shirky 2003b) and cultural studies. In this last field, Zipf's law was used to study the distribution of access to various works in libraries, in commercial sales, and on the Internet. As we will see below, the distribution of popularity of works does not follow Zipf's law exactly (in the statistical sense). However, one can still tune the parameter so that the function reproduces the observed distribution as closely as possible. The resulting match is then often so good that, for practical purposes, the value of $\alpha$ that gives the best fit can be used as a single number characterizing the degree of diversity of attention to works. In other cases, we will have to be cautious because the Zipf's law approximation becomes too distant from what is observed in real-life.

When this diversity is modeled by Zipf's laws, changes in the parameter lead to great differences in the degree to which attention is concentrated on a limited number of works, or sales concentrated on a limited number of products. For 
example, let us consider a sample, or "universe", of one thousand works. If the access to these works is described by Zipf's law with parameter 0.5 , the $5 \%$ most popular works receive $20 \%$ of the attention. If the parameter is 1.5 , the same $5 \%$ accounts for $92 \%$ of all access. Perhaps surprisingly, differences as strong as these are in fact observed between the various forms of access to cultural works.

\section{Why do some distributions follow Zipf's law?}

There have been many attempts at explaining why some distributions observed in real life are very similar to Zipf's law. George Zipf himself suggested that it results from the tendency of human beings to select the least-effort route to any particular result. Here we propose an explanation that builds on the same ideas, though we formulate it slightly differently. However, it is beyond the scope of this book to model the conditions which give rise to Zipf's law in detail: the remarks which follow are merely intended to shed some light on the processes at work.

There are common traits of the kind of variables which follow Zipf's law, such as the level of attention, the usage of or access to works, the income or wealth of individuals, the size of cities, or the occurrences of words in a language. Generally, a variable follows a power law distribution when two conditions are fulfilled:

- its values are constrained by a resource which, while abundant globally, is limited at the level of individual units;

- the larger the variable, the easier it is for it to grow.

Both parts of the first condition are equally important. The constraining resource can be on the offer or on the demand side. There is a lot of wealth overall, but it is not so easy for most individuals to get more of it for themselves. There are many words in a language, but using the rarer ones requires extra effort, for most people at least (in fact, the effort is approximately inversely proportional to the scarcity of each word). ${ }^{3}$ There are many inhabitants in a country, but a given individual can only live in one place. And finally, the overall attention span of Internet users on the planet is certainly not scarce, but each individual has only a limited amount of time. The second condition may be somewhat more self-evident: it is a well-known fact that being rich makes it considerably easier to become richer. Frequently used words are in everybody's mind and get used - to some degree more frequently. This dynamic is most visible when a new buzzword appears. It has limits, however, since one cannot use the same word all the time. Similarly, a cultural work that has already received significant attention is likely to receive more, through word of mouth or through investment in its promotion... up to a certain point where it becomes more difficult to attract the attention of additional persons.

We will now look at finding out if distributions follow Zipf's law, and at methods for estimating the value of their parameter. 


\section{A.2 Testing and parameter estimation for Zipf's law}

When popularity follows Zipf's law, the level of access or usage for each work can be modeled as:

$$
\mathrm{z}(\mathrm{n})=\frac{\mu}{\mathrm{H}_{\mathrm{N}}(\alpha) \times \mathrm{n}^{\alpha}}
$$

where $z(n)$ is the number of times a work of rank $n$ has been accessed or used (works are ranked by decreasing popularity), $\mathrm{N}$ is the total number of works (books in a library, music tracks on a download platform, or blogs on the Internet), $\mu$ is the mean number of accesses to or uses of works, and $\mathrm{H}_{\mathrm{N}}(\alpha)$ is a constant used for normalization. ${ }^{4}$ Specifically, $\mathrm{H}_{\mathrm{N}}(\alpha)$ is the $\mathrm{N}^{\text {th }}$ generalized harmonic number:

$$
\mathrm{H}_{\mathrm{N}}(\alpha)=\sum_{\mathrm{n}=\mathrm{I}}^{\mathrm{N}} \frac{\mathrm{I}}{\mathrm{n}^{\alpha}}
$$

The quantity $\frac{\mu}{\mathrm{H}_{\mathrm{N}}(\alpha)}$ is a constant. Zipf's law is also a power law, but with index $\mathrm{I}+\frac{\mathrm{I}}{\alpha}$ (see Adamic $2002 \mathrm{~b}$ for details). ${ }^{5}$

Checking whether an observed ranked distribution does indeed follow Zipf's law and estimating the best-fit parameter $\alpha$ (the value of $\alpha$ for which Zipf's law most closely resembles the observed distribution) are a bit tricky. The overwhelming majority of published papers on these subjects use a simple property of power laws: when both axes are plotted on logarithmic scales, they appear more or less as a straight line with slope $-\alpha$, where $\alpha$ is the index of the law. Figure 13.3 shows an example, which will give the reader an adequate idea of a statistician's definition of a straight line. It is straightforward to estimate the slope of the line in this log-log space, using techniques such as least-squares regression or log-2 bins. However, (Goldstein et al. 2004) showed that this approach is not only imprecise, but also strongly biased. We devised a fast convergent method for fitting Zipf's law (estimating $\alpha$ ) for an exhaustive observed distribution ${ }^{6}$ which does not suffer from these shortcomings. When only incomplete data is available, which is often the case for commercial access, it is preferable to use the method suggested in (Goldstein et al. 2004). ${ }^{7}$ 


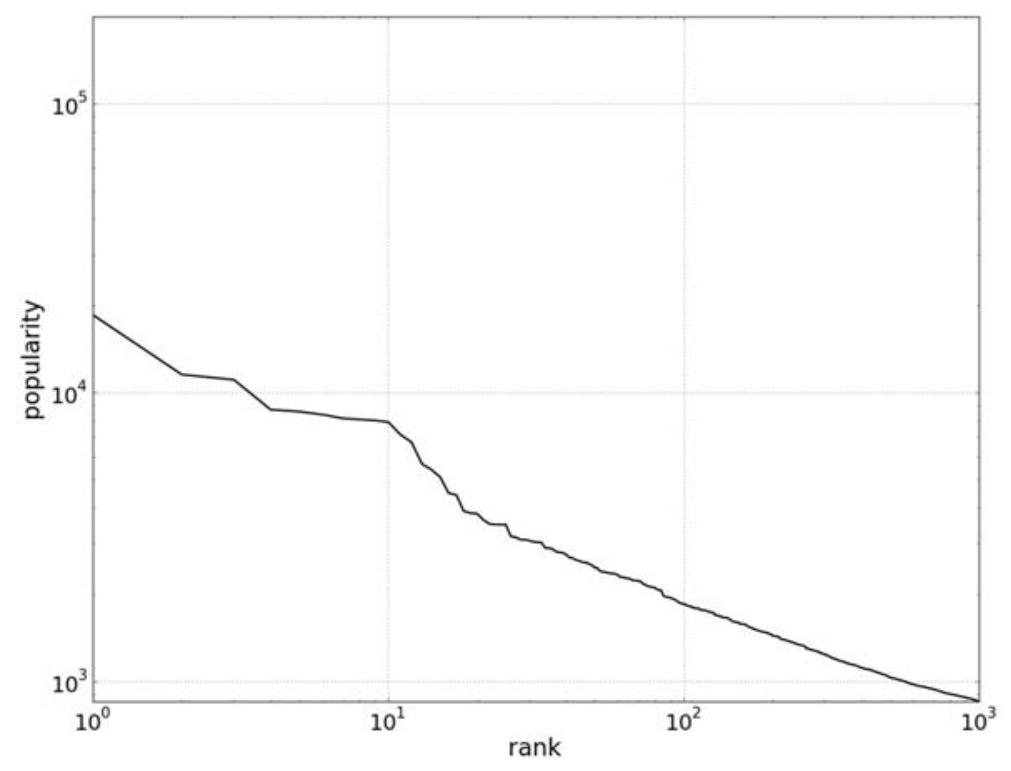

Fig. A.3. Number of times each track was listened to for the 1000 most popular titles on the Musique Libre Creative Commons music streaming plafform in 2006 (Aigrain 2006). Note that both axes are logarithmic.

Once the fitting is done, one can check how closely the resulting Zipf's law matches the data. Rigorous statistical tests typically indicate that the function which gives rise to observed distributions is not exactly a form of Zipf's law. Andrew Odlyzko ${ }^{8}$ remarked that this is quite natural, since different parts of the distribution of attention are influenced by very different factors. In particular, at the extremes of the distribution, phenomena which are unlikely to follow a pure power law pattern become important. A limited number of works benefits from some expensive channels of promotion such as clips, TV, radio or billboard advertising. There can be a significant gap in attention levels between these works and those that follow them in popularity. In $\mathrm{P}_{2} \mathrm{P}$ file sharing, the other end of the distribution is riddled with pollution, some unintentional (user errors), but most intentional (fakes and forged file identifiers injected by $\mathrm{P}_{2} \mathrm{P}$ warfare providers to direct users to irrelevant contents, in order to make them waste time and lose interest in $\mathrm{P}_{2} \mathrm{P}$ file sharing). $\mathrm{P}_{2} \mathrm{P}$ users and supporting sites are good at quickly spotting these polluting elements, and their audience is generally limited, but they are nonetheless accessed a few tens of times. This causes the distribution to lose a key property of power laws: scale invariance, that is the fact that estimating the parameter law can be done on any part of the distribution. However, when 
one limits the analysis to the initial part of the distribution (accounting for 80 or $90 \%$ of all accesses), one obtains a reliable estimate of the parameter, with a better fit. This amounts to selecting only files that actually correspond to works being shared.

In practice, one is not concerned with knowing if popularity exactly follows a Zipf law in the statistical sense, but rather with whether one can use the Zipf's law as an approximate model for reflecting on policy decisions. It turns out that the same distance function that is used for rigorous goodness of fit testing also provides a good indication of the meaningfulness of a Zipf's law approximation for policy reasoning. The Kolmogoroff-Smirnov distance (or KS) distance defines the distance between two distributions as the maximum absolute difference between the value of the cumulative distributions. So if we have a distance of say, 0.03 (3\%) between a popularity distribution and its best-fitting Zipf's law approximation, it means that we can pick any rank, and the total attention going to works after this rank (or before) will not be further from the value we would have estimated using Zipf's law approximation by more than $3 \%$ of the total attention to all works. Now, is that a lot or little? It is a lot, and enough to reject the statistical hypothesis that the distribution follows a Zipf law with great certainty when considering universes of more than Iooo works. But it is little if one uses the approximation only to comment on differences of, say, $20 \%$ of the total attention going to works in a given range of popularity ranks.

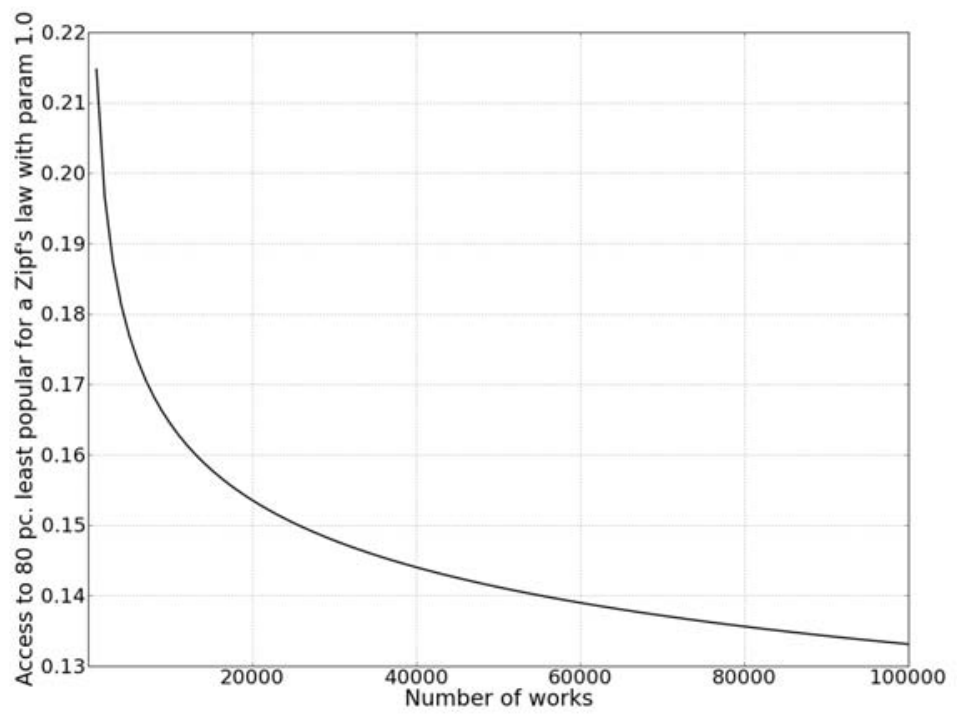

Fig. A.4. Share of access to the 80\% least popular works under Zipf's law with the same parameter ( $\alpha=\mathrm{I} .0$ ) in 100 universes containing between 1000 and 100,000 works 
It is only meaningful to use Zipf's law parameters to compare observed distributions of popularity if both sets of works are of similar size, or with a careful normalization process to compare the two differently sized universes. Forgetting this has led to many errors of interpretation, in particular from critics of the Long Tail theory (see below). The impact of the size of the "universe" on Zipf's law is illustrated in figure A.4: even in this limited range of universe sizes, with the same law (the same parameter $\alpha$ ), the share of access to the $80 \%$ least popular works goes down from more than $21 \%$ to less than $14 \%$.

\section{A.3 Zipf's law and diversity of attention in $\mathrm{P}_{2} \mathrm{P}$ sharing}

Our studies of information communities where authors share works under Creative Commons licenses and of sites providing access to selected public domain works show a good fit of the observed distribution of popularity with the bestfitting Zipf's law. The situation is different with large-scale (predominantly unauthorized) file sharing.

When individuals share works through $\mathrm{P}_{2} \mathrm{P}$ networks, the number of works being shared is enormous. Works that are not offered for commercial access (because rights holders do not think it is in their interest to do so) or orphan works ${ }^{9}$ are in large number, even though each of them might be shared only a limited number of times. During ten weeks in 2008, Frédéric Aidouni, Mathieu Latapy and Clémence Magnien (Aidouni et al. 2009) monitored all traffic going through one of the servers used by the eDonkey $\mathrm{P}_{2} \mathrm{P}$ network. This data was strongly anonymized and made publicly available. The collected data represents close to 9,000 million eDonkey messages, involving around 9o million distinct client IDs. No fewer than 3,000 million file swaps flowed through this specific server during those ten weeks. Just under 12 million distinct files were shared, each of them being accessed more than 250 times on average. The data collected on these exchanges (excluding the files themselves) amounts to close to Io terabytes (I0,000 gigabytes) in uncompressed form, which is unprecedented for publicly available data relevant to Internet studies. We are deeply indebted to Mathieu Latapy's Complex Networks research team at the LIP6 laboratory of Paris 6 University for having collected and published this data at http://data.complexnetworks.fr/ weeks-I.0.I/.

The collection of the data was possible because of guarantees provided by the collectors on the strength of the anonymization process. This has some consequences for analyzers: as not only user information but also work information has been anonymized, it is impossible to reliably identify works that belong to a given medium. The results of our analysis should thus be considered as relevant to the general study of diversity of attention in file sharing, rather than to the study of a given medium. 
We analyzed the data in collaboration with Raphaël Badin, with the assistance of Suzanne Aigrain, and thanks to the use of the University of Exeter supercomputer facility. In the eMule protocol implemented by the eDonkey server, users go through two steps: they first query the list of available works, using keywords such as an artist name, a title, or a file name or size, and they obtain in response a list of available files. A user then selects one or several of the files and asks for sources (peers) providing these files. As soon as the sources have been obtained, the download starts. It is the requests for sources that provide an indication of access to the corresponding work. We thus extracted the occurrences of file identifiers in the answers to queries for sources. ${ }^{\mathrm{IO}}$ We counted the occurrences for each unique identifier, and sorted the identifiers by decreasing number of occurrences, in order to obtain the observed popularity distribution. We then ran our fitting algorithm to obtain the best-fitting Zipf's law. Table A.I lists the best-fit parameters and the corresponding KS distance on cumulative distributions (which measures the quality of the fit, the smaller the better) for different subsets of the data.

As already remarked in (Aidouni et al. 2009), the tail of the distribution is extremely polluted: the fit becomes poor when using the one million most popular files or more. On the basis of the data presented in (Aidouni et al. 2009) and (Lee et al. 2006), we are reasonably certain that the deviation at the end tail of the distribution is predominantly due to the intentional pollution of $\mathrm{P}_{2} \mathrm{P}$ networks. Further research would be needed to definitely prove this hypothesis, for instance by precisely modeling the noise introduced by fakes.

\begin{tabular}{lcccc} 
Universe & $\begin{array}{l}\text { Parameter of } \\
\text { best fitting } \\
\text { Zipf's law }\end{array}$ & $\begin{array}{l}\text { KS distance on } \\
\text { cumulative } \\
\text { distributions }\end{array}$ & $\begin{array}{l}\text { More access } \\
\text { observed from } \\
\text { rank ... }\end{array}$ & to rank \\
\hline $\begin{array}{l}\text { 100,000 distinct most popular } \\
\text { distinct files }\end{array}$ & 0.378 & 0.0056 & 4,830 & 62,100 \\
$\begin{array}{l}\text { One million distinct most popu- } \\
\text { lar files (85\% of recorded access) }\end{array}$ & 0.581 & 0.0645 & 18,000 & 491,000 \\
$\begin{array}{l}\text { Two million distinct most popu- } \\
\text { lar files (95\% of recorded access) }\end{array}$ & 0.682 & 0.1073 & 18,800 & 750,000 \\
$\begin{array}{l}\text { One-third of all files (close to } 4 \\
\text { million files) }\end{array}$ & 0.758 & 0.1496 & 17,700 & $1,050,000$ \\
$\begin{array}{l}\text { Full set of files (close to 12 mil- } \\
\text { lions files) }\end{array}$ & 0.842 & 0.2079 & 15,000 & $1,551,000$ \\
\hline
\end{tabular}

Table A.1. Zipf's law fitting for different subsets of the (Aidouni et al. 2009) data set. The last two columns give the range of ranks for which the observed access is higher than the bestfitting Zipf's law prediction. 
In figure 3.4, we analyzed the diversity of attention for the two million most popular files, which represented $95 \%$ of recorded access, and provide a universe of similar size to the one studied for commercial sales of music in (Page-Garland 2009). Considering the excessive deviation from Zipf's law, we did not use a Zipf law approximation to compare the $\mathrm{P}_{2} \mathrm{P}$ situation with the available estimates regarding commercial access to music, but rather the directly observed data. One should note that the observed distribution is actually more diverse than its bestfitting Zipf's law: the observed popularity is above the best-fitting Zipf's law for popularity ranks ranging from 18,800 to 750,000 . We already observed this for voluntary sharing communities (Aigrain 2006).

Important notice: We presented preliminary results of this study at the 2009 Free Culture Research Workshop at Harvard University (Aigrain-Badin 2009). They were based on a different analysis of the data collected by the Paris 6 team: instead of analyzing the access to source files, we analyzed the occurrences of files in answer to keyword queries. This analysis found an even greater degree of diversity. However, it is less representative of the real-life usage of works, in our opinion.

\section{A.4 A fresh look at the Long Tail theory}

In 2004, Chris Anderson published his famous Long Tail article (Anderson 2004). This article forecast that the Internet would lead to more diversity of attention, but stopped short of modeling this increase in detail. The article was expanded into a book in 2006 (Anderson 2006). The same year, other researchers (Brynjolsson et al. 2006) provided the first quantitative evidence for an increased diversity of sales in the Internet channel for products also marketed in physical channels. However, the increase was moderate: in a universe of 20,000 products, it would correspond to a Zipf law parameter of 0.877 instead of 0.935 .

In the years that followed, a number of critics challenged the accuracy of the Long Tail theory or its relevance to business. A typically critical view was expressed in a research working paper by Tom F. Tan and Serguei Netessine of the Wharton Business school (Tan-Netessine 2009). These authors studied the movie ratings from the Netflix on-line movie rental company. The data presented by the Wharton researchers provides an interesting test case and has led to a polite argument with Chris Anderson. Most critics of the Long Tail theory are not contesting its accuracy, but challenging the feasibility of making a successful business out of the increased demand for limited popularity titles. The Wharton researchers went a bit further: they distinguished between the "absolute popularity" (how much attention a work with a given rank receives), which they acknowledged to be the core concern of the Long Tail theory, and a "relative popularity" expressed as the share of attention which a given percentage of all available movies receives. They 
then conclude from their data that the relative share of the top $20 \%$ movies, for instance, has increased over time from $86.6 \%$ to $90.08 \%$.

From the viewpoint of the analysis of diversity of attention, this calls for a number of remarks. First of all, the comparison is between $86.6 \%$ of 4,470 movies in 2000 and $90.08 \%$ of 17,468 movies in 2005 . We can use our earlier experiments on the impact of universe size on the share of attention going to the $20 \%$ most popular works to explore the impact of this growth in the number of movies available. Keeping the Zipf's law parameter at I.075, the initial value, we find that the share of interest would rise from $86.6 \%$ to $89.1 \%$. In other words, the "effect" observed by the Wharton researchers would have occurred even if the underlying law had not changed at all because of the behavior of Zipf's law when the universe of works expands. More importantly, as Chris Anderson remarked in an email made public on the Web (Anderson 2009), from a cultural diversity perspective, what is important is the share of attention received by the titles of intermediate absolute popularity rank, for instance those ranked between 1000 and 4000: films which are not hits, but are still popular. Taking 2002 as a starting point (there were not enough rated movies to study this popularity range before that), we find that the proportion of ratings going to this range of absolute ranks went up from at most $12 \%$ to at least $28 \%$ of the total.

These intermediate popularity works may not receive enough attention to be conducive to classical commercial activity (revenues from sales or rentals). However, there is no doubt that they can be rewarded by an indirect scheme such as the Creative Contribution.

What remains to be shown is whether the corresponding level of attention can be measured accurately enough. This is the object of appendix C. 


\section{B The total cost of rewards and their distribution}

\section{B.1 The model used in chapter 7}

\section{Evolution of the distribution of attention}

In chapter 7 , we attempted to describe the compound result of the autonomous development of an Internet-native culture production and of the continued usage of the Internet as a distribution medium for media publishing. The interaction of these factors leads to a popularity distribution for creators (beneficiaries of the Creative Contribution), which is the basis for our model.

To explain what we mean by "popularity distribution for creators", we need to define a few quantities. If each work $\mathrm{w}_{\mathrm{j}}$ has popularity $\operatorname{pop}\left(\mathrm{w}_{\mathrm{j}}\right)$, and the relative contribution of a given creator $c_{i}$ to $w_{j}$ is contrib $\left(c_{i}, w_{j}\right)$, the total popularity ${ }^{r}$ for a given creator is:

$$
\operatorname{pop}\left(\mathrm{c}_{\mathrm{i}}\right)=\sum_{\mathrm{j}} \operatorname{contrib}\left(\mathrm{c}_{\mathrm{i}}, \mathrm{w}_{\mathrm{j}}\right) \times \operatorname{pop}\left(\mathrm{w}_{\mathrm{j}}\right)
$$

For example, if creator A contributed $50 \%$ of work $\mathrm{w}_{\mathrm{I}}, 25 \%$ of work $\mathrm{w}_{2}$ and $60 \%$ of work $\mathrm{w}_{3}$, the popularity of $\mathrm{A}$ is:

$$
\operatorname{pop}(A)=0.5 \operatorname{pop}\left(w_{\mathrm{I}}\right)+0.25 \operatorname{pop}\left(w_{2}\right)+0.6 \operatorname{pop}\left(w_{3}\right) .
$$

The popularity distribution for creators is the distribution of $\operatorname{pop}\left(\mathrm{c}_{\mathrm{i}}\right)$ : it determines the distribution of the rewards. We will speak equivalently of usage distribution, because our measurement system collects usage clues (see appendix C).

A number of heterogeneous factors (such as classical media publishing and unauthorized file sharing) already interact to drive the attention devoted to various productions. This interaction will be stronger once the Creative Contribution is put in place and file sharing is recognized as a legitimate activity. It will result - as it already does - in attention patterns (i.e. popularity distributions) that do not formally follow Zipf's law but are often closely approximated by it. We will discuss below the impact of this possible divergence, but for the time being, let us accept modeling the reported usage pattern by a Zipf law and consider only how the associated parameter - and thus the diversity of usage - will vary. 
Note that actual rewards will be distributed according to the observed distribution, not the model: this model is used only to determine the overall amount of rewards, i.e. to set a global scale factor. To do this, we will assume that the input to the reward system will be a Zipf law with parameter $\alpha$. This leaves us with 3 parameters to set: the value of $\alpha$, the size of the overall universe (the number of creators to be rewarded), and the minimum reward amount to be distributed.

As explained in section 7.2, one can only speculate about the likely observed value of $\alpha$. In chapter 7, we predict that it will start at the high value of I.O (the classical Zipf law, but actually less concentrated than present copyright rewards), and then progressively decrease to 0.9 (a more diverse distribution of attention), possibly becoming as low as 0.8 in the long term. The interested reader is referred to figure 7.2 for a comparison between the corresponding distributions. ${ }^{2}$ Given the uncertainty of $\alpha$ and the likelihood that it will vary, it is important to build a model which can function at constant total cost regardless of the precise value of $\alpha$, at least for the predictable future.

\section{The reward level and size parameters}

To set the remaining two parameters, we start from the decision that a certain number of people should be rewarded at or above a certain minimum level in a given country, at the time when the Creative Contribution is introduced. This choice is not arbitrary, but it is clearly based on a programmatic decision rather than on some more fundamental principle: there is no obvious ground truth that immediately tell us that "we should reward so many people by at least this amount".

We chose to set this minimum reward at $\$ 200 / y e a r$ (or the local currency equivalent), and the number of people who should receive it at 2-2.5\% of individual Internet contributors who "produce and publish some contents for sharing over the Internet in a 3 month period" (See Deroin 2010), and we did our best to substantiate this decision (see page 100), but we acknowledge that a different choice could be made. However, there are some important constraints. The number of creators to be rewarded at the minimum level cannot be raised arbitrarily, not only because it would make the reward system too expensive, but also because it is not clear that there are enough deserving creators out there to justify it, or that they would come out of the woodwork if a reward was available.

All the computer programs used to experiment with our model are distributed in parallel with the publication of this book as free software ${ }^{3}$, and readers are encouraged to experiment with other possible values, should they wish to do so.

Our choice for the threshold below which rewards will not be distributed is \$40/year (justified on page 99). Once these 2 choices have been made, the other decisions follow for a given value of the diversity parameter $\alpha$. 
Setting an initial value for the universe size

For the time being, let us assume a proportional reward, where creators are rewarded proportionally to the measured usage of their works. Let's assume that the value of the parameter of Zipf's law for the initially observed diversity of usage will be $\alpha=\mathrm{I} .0$, and we wish to have at least $\mathrm{n}=230$, 000 creators receiving $€ 150 /$ year or more. The following formula immediately gives the total number of rewarded creators

$$
\mathrm{N}=\exp \left[\ln (\mathrm{n})+\frac{\mathrm{I}}{\alpha} \ln \left(\frac{\mathrm{I50}}{30}\right)\right]
$$

where $\exp (\mathrm{x})$ is the exponential of $\mathrm{x}$ and $\ln (\mathrm{n})$ is the natural logarithm of $\mathrm{n}$. The formula is obtained as follows. According to Zipf's law, the reward for the $\mathrm{n}^{\text {th }}$ creator is:

$$
\operatorname{reward}(\mathrm{n})=\frac{\mathrm{R}_{\alpha}}{\mathrm{n}^{\alpha}}
$$

where $\mathrm{R}_{\alpha}$ is a constant, or scale factor, that sets the overall level of the rewards. The last creator being rewarded (the one which receives the smallest amount) receives:

$$
\operatorname{reward}_{\min }=\frac{\mathrm{R}_{\alpha}}{\mathrm{N}^{\alpha}}
$$

Dividing one equation by the other:

$$
\frac{\operatorname{reward}(\mathrm{n})}{\text { reward }_{\min }}=\frac{\mathrm{N}^{\alpha}}{\mathrm{n}^{\alpha}}
$$

Now take the natural logarithm of both sides:

$$
\ln \left[\frac{\operatorname{reward}(\mathrm{n})}{\text { reward }_{\min }}\right]=\alpha \ln (\mathrm{N})-\alpha \ln (\mathrm{n})
$$

rearrange:

$$
\ln (\mathrm{N})=\ln (\mathrm{n})+\frac{\mathrm{I}}{\alpha} \ln \left[\frac{\operatorname{reward}(\mathrm{n})}{\text { reward }_{\min }}\right]
$$

and take the exponential of both sides:

$$
\mathrm{N}=\exp \left\{\ln (\mathrm{n})+\frac{\mathrm{I}}{\alpha} \ln \left[\frac{\text { reward }(\mathrm{n})}{\text { reward }_{\text {min }}}\right]\right\}
$$

Plugging in the values $\operatorname{reward}(\mathrm{n})=150, \operatorname{reward}_{\min }=30, \mathrm{n}=230,000$, and $\alpha=\mathrm{I}$ gives:

$$
\mathrm{N}=\exp \left\{\ln (230,000)+\frac{\mathrm{I}}{\mathrm{I}} \ln \left[\frac{\mathrm{I} 50}{30}\right]\right\}
$$




$$
\begin{gathered}
=\exp \{\ln (230,000)+\ln (5) \\
=\exp \{\ln (230,000 \times 5)=\mathrm{I}, \mathrm{I} 50,000
\end{gathered}
$$

\section{Running the model}

All we now need is to work out the total reward, that is, the sum of all the rewards:

$$
\begin{gathered}
\operatorname{reward}_{\mathrm{tot}}=\sum_{\mathrm{m}=\mathrm{I}}^{\mathrm{N}} \operatorname{reward}(\mathrm{m})=\sum_{\mathrm{m}=\mathrm{I}}^{\mathrm{N}} \operatorname{reward}_{\min } \frac{\mathrm{N}^{\alpha}}{\mathrm{m}^{\alpha}} \\
\text { reward }_{\text {tot }}=\operatorname{reward}_{\min } \times \mathrm{N}^{\alpha} \times \sum_{\mathrm{m}=\mathrm{I}}^{\mathrm{N}} \frac{\mathrm{I}}{\mathrm{m}^{\alpha}} \\
\operatorname{reward}_{\text {tot }}=\operatorname{reward}_{\min } \times \mathrm{N}^{\alpha} \times \mathrm{H}_{\mathrm{N}}(\alpha)
\end{gathered}
$$

where $\mathrm{H}_{\mathrm{N}, \alpha}$ is the $\mathrm{N}^{\text {th }}$ harmonic number, already introduced in Appendix A. This formula immediately gives the total reward, the only step that requires some simple assistance is the computation of $\mathrm{H}_{\mathrm{N}}(\alpha)$.

\section{How many creators are rewarded as diversity increases?}

If we are now in a situation where the observed diversity of use corresponds to another value of Zipf's law parameter, say $\alpha$ ' we can find the new number of rewarded creators N' that will lead to the same total cost in this new situation:

$$
\mathrm{N}^{\prime \alpha^{\prime}} \times \mathrm{H}_{\mathrm{N}}{ }^{\prime}\left(\alpha^{\prime}\right)=\mathrm{N}^{\alpha} \times \mathrm{H}_{\mathrm{N}}(\alpha)
$$

Solving this equation for $\mathrm{N}^{\prime}$ is not that trivial, and is easier done by approximation techniques or using numerical tables. For instance in the example above with $\mathrm{N}=\mathrm{I}, \mathrm{I50}, 000$ and $\alpha=\mathrm{I} .0$, the solution corresponding to $\alpha=0.9$ is $\mathrm{N}^{\prime}=2, \mathrm{I} 4 \mathrm{O}, 000$ and for $\alpha=0.8$ it is $\mathrm{N}^{\prime}=3,490,000$.

\section{Impact of a divergence of the observed usage from Zipf's law}

Only when a measurement system is fully in place can we judge if the reported usage follows Zipf's law. What are the consequences if it does not? We have designed a constant cost reward system, and this cost can be distributed according to the observed usage, but with some adjustments.

If we want to keep the minimal reward and still distribute the same total amount of rewards, we will have two differences in comparison with what would have happened with the Zipf law fitted to the observed usage:

- the number of rewardees will be different; 
- the level of use corresponding to the minimal reward will be different.

The second effect could be the most problematic one if it forces us to measure usage precisely at much lower levels than modeled in appendix C. Fortunately, this is easy to avoid so long as one does not try to reward an excessive number of creators, staying clear of the level of attention where real use is mixed with noise. If we take the example of the 2 million most popular files in ro weeks of usage of eDonkey $\mathrm{P}_{2} \mathrm{P}$, where we have a significant divergence between the best-fitting Zipf's law and the observed data (see table I3.I), the level of usage for the $\mathrm{I}, 000,000^{\text {th }}$ most popular work is only $24 \%$ lower in the observed data than in the model.

\section{B.2 Reward functions}

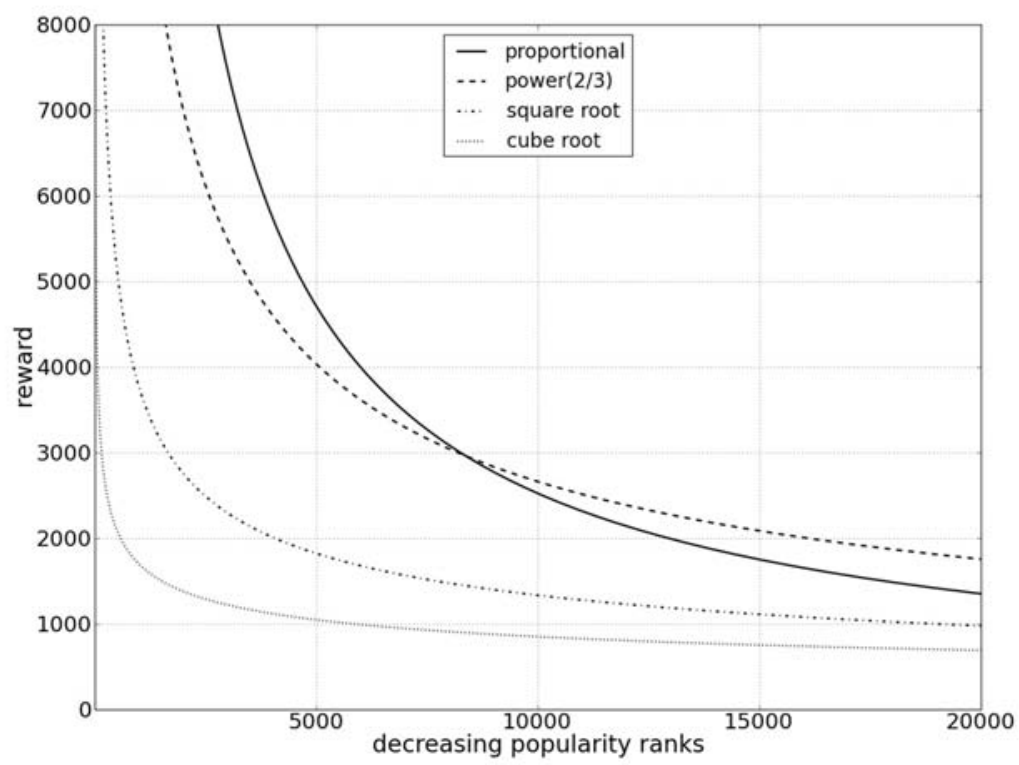

Fig. B.1. Rewards for the first 20,000 creators among one million rewarded creators for various reward functions, with a creator popularity distribution corresponding to a Zipf law parameter of 0.9 , and a minimum reward of $\$ 40$. The cube root reward function was suggested by Richard Stallman (Stallman 2009). If a non-proportional reward can be implemented, we favor the choice of a power law reward function with index $2 / 3$, associated with a topoff for the highest rewards. 
Now we relax the assumption that the relation between popularity and reward is necessarily proportional. This is not unusual: in most media publishing, the remuneration for creators is more than proportional to sales. A bestselling author might get four times the percentage of royalties of an average essay writer, a fact explained in part by economies of scale in the production and distribution of books, but mainly by the stronger negotiating power of bestselling authors. In the digital world, there are strong fairness and diversity motives for using lessthan-proportional rewards.

To allow for this, we introduce a reward function reward $(n)=r\left(p_{n}\right)$, where $p_{n}$ is the popularity of creator $\mathrm{n}$ relative to that of the least popular creator who will be rewarded: $p_{n}=\operatorname{pop}\left(c_{n}\right) / \operatorname{pop}\left(c_{N}\right)$. The reward is proportional if $r\left(p_{n}\right)=p_{n}$. If we want the reward to be proportional to the square root of popularity, we would use $\mathrm{r}\left(\mathrm{p}_{\mathrm{n}}\right)=\sqrt{\mathrm{p}_{\mathrm{n}}}$.

The total reward is now given by:

$$
\operatorname{reward}_{\mathrm{tot}}=\operatorname{reward}(\mathrm{N}) \times \sum_{\mathrm{n}=\mathrm{I}}^{\mathrm{N}} \mathrm{r}\left(\frac{\mathrm{N}^{\alpha}}{\mathrm{n}^{\alpha}}\right)
$$

In the case of a square root reward:

$$
\operatorname{reward}_{\text {tot }}=\operatorname{reward}_{\min } \sum_{\mathrm{n}=\mathrm{I}}^{\mathrm{N}} \sqrt{\frac{\mathrm{N}^{\alpha}}{\mathrm{n}^{\alpha}}}=\operatorname{reward}_{\min } \mathrm{N}^{\frac{\alpha}{2}} \mathrm{H}_{\mathrm{N}}\left(\frac{\alpha}{2}\right)
$$

In other terms, square root rewards for a distribution of parameter $\alpha$ are the same as proportional rewards for a distribution of parameter $\frac{\alpha}{2}$. The same holds for any power reward function. 


\section{Modeling usage measurement}

This appendix details the model from which affirmations on the precision reachable by the voluntary sample of Internet users component of our proposed measurement system were derived.

\section{C.1 General model}

Let us first remark on a nice property of the constant cost reward system we defined in chapter 7 and detailed in appendix B. The minimum relative level of use (for all works by a person) that we need to measure is:

$$
\mathrm{U}_{\mathrm{N}}=\frac{\mathrm{I}}{\mathrm{H}_{\mathrm{N}}(\alpha) \mathrm{N}^{\alpha}}
$$

where $\mathrm{N}$ is the number of contributors we want to reward above the set threshold, and $\mathrm{H}_{\mathrm{N}(\alpha)}$ is the $\mathrm{N}^{\text {th }}$ harmonic number for parameter $\alpha$. This is precisely the figure we made constant in order to define a constant cost reward system in equation in section B.I. It thus seems that we can completely forget about how the observed diversity will vary (or which power reward function we will adopt): the challenge of measuring the minimum use that leads to a reward remains the same. Unfortunately, for sake of rigor, we must consider a number of contextual factors that force us to depart from this beautiful simplicity, at least if we are going to distribute rewards independently for various media.

The first step is to find out for how many works we need the system to be precise enough. The reward system as a whole has been calibrated to reward a certain number of creators. From the processes described in chapter 9, we will decide which share goes to each medium. From this, one can obtain a number $\mathrm{R}$ of creators to be rewarded for the medium under study. At the time being, we have to estimate this figure approximately: remember that we are just trying to figure out which measurement system can work, not yet to actually distribute rewards. When we will reach that stage, we will know the figure precisely. For music in the US, as an example, we estimated that a quarter of all rewardees would be music artists at large, which gives us $\mathrm{R}=\mathrm{I}, 050$, 000 rewardees above the minimum amount of $\$ 40$. We consider that the diversity of attention to creators in music is the same as that in the overall system when put in place (parameter of Zipf's law = I.0). 
We must then take into account the fact that creators are involved in several works, and a work can have several creators. The compound result of the two will be an estimate of the average number of works one needs to reward in order to meet a stated objective of rewarding a number of creators. For instance, for music, we estimated that one needs to reward $\mathrm{N}=3 \times \mathrm{R}$ works in order to reward $\mathrm{R}$ creators, that is $\mathrm{N}=3, \mathrm{I} 50,000$. This leads to approximation: to derive a minimal reward, titles that are below a third of $\$ 40$ will be used. We will have to consider this when interpreting the results. ${ }^{\text {I }}$

Finally, we must acknowledge that not all relevant works will be registered for rewards. We define

$$
\mathrm{N}^{\prime}=\mathrm{kN}
$$

where $\mathrm{k}$ is a coefficient ensuring that within the $\mathrm{kN}$ most popular works, at least $\mathrm{N}$ will be registered. For instance, for music, we predicted that almost all works likely to lead to creator rewards will be registered for rewards ( $k=\mathrm{I} .2$, and $\left.\mathrm{N}^{\prime}=3,780,000\right)$.

We can now proceed to our main task: estimating the number of use clues that will be received from the full sample of Internet users for works registered for rewards and positioned at various levels of popularity.

In order to do so, we estimate the number of clues that would be reported on average by a member of the voluntary sample for a registered work, based on statistics of the average use of an individual or the average use of works, depending on which is more readily available.

We first consider an ideal situation in which all users would be members of the sample. We have

$$
\eta \times \mathrm{N}^{\prime}=\theta \times \mathrm{K}=\text { totclues }
$$

where $\eta$ is the average number of clues reported for one work, N' the number of works, $\theta$ the average number of clues reported by one user in a year, $\mathrm{K}$ the number of users, and totclues the total number of clues.

If we know $\eta$, the share of the total number of clues $\mathrm{C}(\mathrm{n})$ that will be reported for a work whose popularity rank is $\mathrm{n}$ in the medium under consideration is

$$
\mathrm{s}(\mathrm{n})=\frac{\eta \times \mathrm{p}}{\mathrm{H}_{\mathrm{N}}(\alpha) \times \mathrm{n}^{\alpha}}
$$

where $\alpha$ is the parameter of Zipf's law of the popularity distribution, and $\mathrm{p}$ is the proportion of all use of the same set of works coming from participants in the sample. The absolute number of clues will be:

$$
\mathrm{C}(\mathrm{n})=\mathrm{s}(\mathrm{n}) \times \mathrm{N}^{\prime}=\frac{\eta \times \mathrm{p} \times \mathrm{N}^{\prime}}{\mathrm{H}_{\mathrm{N}}{ }^{\prime}(\alpha) \times \mathrm{n}^{\alpha}}
$$


As we will see below, it is frequent for use of some media to be significantly higher for the likely participants in the sample.

If we know the average use for an individual, we can use equation to compute the average number of clues per work:

$$
\eta=\frac{\theta \times \mathrm{K}}{\mathrm{N}^{\prime}}
$$

and then compute $\mathrm{C}(\mathrm{n})$ as follows:

$$
\mathrm{C}(\mathrm{n})=\frac{\frac{\theta \times \mathrm{K}}{\mathrm{N}^{\prime}} \times \mathrm{p} \times \mathrm{N}^{\prime}}{\mathrm{H}_{\mathrm{N}},(\alpha) \times \mathrm{n}^{\alpha}}=\frac{\theta \times \mathrm{p} \times \mathrm{K}}{\mathrm{H}_{\mathrm{N}},(\alpha) \times \mathrm{n}^{\alpha}}
$$

where $\mathrm{p}$ is the proportion of use of the same set of works coming from participants in the sample of all users.

Finally, when the number of clues for a work contributing to a minimal reward is known, we have to go back to the clues for one creator (3 times more in the music example).

Overall, we are assuming that:

I. the popularity distribution of works will actually be well-fitted by a Zipf law

2. we predicted the Zipf law parameter correctly,

3. the average number of clues for a given level of use is as we estimated,

4. the number of registered works is as predicted.

That's a lot of conditions, but conditions 2 and 4 can be checked when the actual numbers are in, in order to possibly adjust the number of rewarded works or choose an adequate reward function.

Under these assumptions, we can estimate the precision with which the number of reported clues will follow this theoretical model: the central limit theorem ${ }^{2}$ guarantees us that for large distributions (typically when $\mathrm{C}(\mathrm{n})>\mathrm{Io0}$ ), the number of reported clues for a work at a given popularity will be well approximated by a normal law with mean $\mathrm{C}(\mathrm{n})$ and standard deviation $\sqrt{\mathrm{C}(\mathrm{n})}$. This means that the probability of deviation greater than $2 / \sqrt{\mathrm{C}(\mathrm{n})}$ is below $5 \%$.

\section{C.2 Music singles in the US}

As explained above, we estimate that the number of works for which a usage measurement has to be derived is $\mathrm{N}^{\prime}=3,780,000$. One can be surprised at this figure when there are 13 million tracks in iTunes alone. ${ }^{3}$ Remember however that we are considering only titles likely to be rewarded more than a third of $\$ 40$. Our own estimate is that only for about 2,100,000 iTunes titles do sales lead to a distribution of more than $\$ \mathrm{I}_{3}$ to creators. ${ }^{4}$ 
We will use two sources of information in order to estimate how many use clues we are likely to get from the voluntary user sample: listening time statistics and digital sales.

For contents for which there is a significant commercial activity, statistics about the annual time use of users are generally available. The US Census Bureau 2010 Statistical Yearbook, table I094, reports 177 h per person of listening to recorded music per year, that is $I_{5} \times I_{77}=2655$ listenings to 4 minute tracks per year. This figure summarizes music listening only when it is done as a main activity, while music listening is very frequently a secondary activity, particularly in the youth. A gross estimate is that at least a quarter of listening occurs with shared files. However, there are several downloads per listening in the file sharing universe. In addition, heavy Internet users spend three times more time listening to music than the average member of the population. ${ }^{5}$ Our estimate is that the average number of clues reported if all users were included in the sample would be similar to the average number of listenings as reported by the time use statistics, that is $\theta=2655$. We will take the proportion of all use of the same set of works coming from participants in the sample to be twice the proportion of users participating in the sample. As we consider the initial sample to be $\mathrm{I} / 400^{\text {th }}$ of all broadband Internet users in the US, we will have $\mathrm{p}=\mathrm{I} / 200=0.005$. We will take the total number of users to be $\mathrm{K}=\mathrm{I} 60$, , oo , 000 .

We then apply our model

$$
\begin{gathered}
\mathrm{C}(\mathrm{n})=\frac{\theta \times \mathrm{p} \times \mathrm{K}}{\mathrm{H}_{\mathrm{N}},(\alpha) \times \mathrm{n}^{\alpha}} \\
\mathrm{C}(\mathrm{n})=\frac{2655 \times 0.005 \times \mathrm{I} 60,000,000}{\mathrm{H}_{3,780,000}(\mathrm{I} .0) \times \mathrm{n}^{\mathrm{I} .0}} \\
\mathrm{C}(\mathrm{n})=\frac{2, \mathrm{I} 24,000,000}{\mathrm{H}_{3,780,000}(\mathrm{I} .0) \times \mathrm{n}}
\end{gathered}
$$

In particular:

$$
\begin{aligned}
& \mathrm{C}(\text { significant })=\frac{2, \mathrm{I} 24,000,000}{\mathrm{H}_{3,780,000}(\mathrm{I} .0) \times 756000} \sim \mathrm{I} 79 \\
& \mathrm{C}(\text { minimal })=\frac{2, \mathrm{I} 24,000,000}{\mathrm{H}_{3,780,000}(\mathrm{I} .0) \times 3,780,000} \sim 36
\end{aligned}
$$

The number of clues per creator is then simply obtained by multiplying the above numbers by 3: (536 and 107).

It is useful to cross that information with analyzing digital sales. In the last year for which data is available, 3,60o million iTunes tunes were sold, which represents 277 sales per title on average. Assuming a distribution of popularity with a 
Zipf law parameter of I.o, this would correspond to an average of approximately 700 sales for the 3,780, 000 most popular titles. With a ratio of Ioo files shared in a year per digital sale, we would have thus an average of $\eta=70$, 000 clues per work if all Internet users were included in the voluntary sample. We will apply our model again with the hypothesis that music listeners and file sharers will be more represented in the voluntary sample $(\mathrm{p}=0.005)$ :

$$
\mathrm{C}(\mathrm{n})=\frac{\eta \times \mathrm{p} \times \mathrm{N}^{\prime}}{\mathrm{H}_{\mathrm{N}^{\prime}}(\alpha) \times \mathrm{n}^{\alpha}}
$$

The number of clues for all users will be

$$
\begin{gathered}
\mathrm{C}(\mathrm{n})=\frac{70,000 \times 0.005 \times 3,780,000}{\mathrm{H}_{3,780,000}(\mathrm{I} .0) \times \mathrm{n}^{\mathrm{I} .0}} \\
\mathrm{C}(\mathrm{n})=\frac{350 \times 3,780,000}{\mathrm{H}_{3,780,000}(\mathrm{I} .0) \times \mathrm{n}}
\end{gathered}
$$

In particular, if we look at the number of clues for the least popular of the works being rewarded at a significant level and the number of clues for the least popular of all rewarded works:

$$
\begin{aligned}
& \mathrm{C}(\text { significant }) \sim \mathrm{III} \\
& \mathrm{C}(\text { minimal }) \sim 22
\end{aligned}
$$

Going back to creators, we have more or less 3 times more clues from the $\mathrm{P}_{2} \mathrm{P}$ file sharing data coming from the sample (333 and 66).

These estimates are obviously approximate and should be considered as indicative only.

\section{C.3 Blogs in France}

Though there are collective blogs and some people have several blogs, overall one blog $=$ one person, which will simplify our modeling. As explained above in section 10.4, we estimated the number of blogs to be rewarded at N $=57,500$ and considered that the registration rate could be relatively low $(k=2$, $\mathrm{N}^{\prime}=\mathrm{II} 5,000$ ). In addition, the diversity of attention to blogs is higher than for other media, as they already anticipate the more diverse distribution which we described as a longer-term perspective in chapter 7 , that is corresponding to a Zipf law of parameter $\alpha=0.8 .^{6}$

To our knowledge, there are no reliable statistics available on the blog reading time use or number of readings for the full population of Internet users. We thus have to turn to the average readership per blog. There are many contradictory claims of the average number of readings per blog, but since we are considering 
here only the II5,000 most appreciated blogs (top $4.5 \%$ of all active blogs), it is reasonable to assume for the number of readings per year to be at least 20,000. ${ }^{7}$ When the blog under consideration is registered, we will assume 1.5 use clues per reading by a member of the voluntary sample, thus on average $\eta=30$, ooo use clues per year. Again, we consider that the original sample of $\mathrm{I} / 400^{\text {th }}$ of all broadband Internet users, that is approximately 100,000 users, will account for twice its share of total blog reading, as participation in the sample will appeal to the same users. We thus have $\mathrm{p}=\mathrm{r} / 200=0.005$.

We are now ready to apply our model:

$$
\begin{gathered}
\mathrm{C}(\mathrm{n})=\frac{\eta \times \mathrm{p} \times \mathrm{N}^{\prime}}{\mathrm{H}_{\mathrm{N}^{\prime}}(\alpha) \times \mathrm{n}^{\alpha}} \\
\mathrm{C}(\mathrm{n})=\frac{30,000 \times 0.005 \times \mathrm{II} 5,000}{\mathrm{H}_{\mathrm{II} 5,000}(0.8) \times \mathrm{n}^{0.8}} \\
\mathrm{C}(\mathrm{n})=\frac{\mathrm{I7}, 250,000}{\mathrm{H}_{\mathrm{II} 5,000}(0.8) \times \mathrm{n}^{0.8}}
\end{gathered}
$$

In particular:

$$
\begin{gathered}
\mathrm{C}(\text { significant })=\frac{\mathrm{I} 7,250,000}{\mathrm{H}_{\mathrm{I}, \mathrm{I} 50,00}(0.8) \times 23,000^{0.8}} \sim \mathrm{II} 8 \\
\mathrm{C}(\text { minimal })=\frac{\mathrm{I} 7,250,000}{\mathrm{H}_{\mathrm{II}, 000}(0.8) \times \mathrm{II} 5,000^{0.8}} \sim 32
\end{gathered}
$$

\section{C.4 Fraud prevention and detection}

Precisely detailing the direct cryptographic anti-fraud mechanisms goes beyond our competence and would be to some extent pointless, as they are bound to evolve. We sketch some possible measures for indicative purposes only assuming that in an option where participants accept that the data collection agency will have knowledge (at the time of reception) of who is reporting, but trust that this identifying information will be immediately deleted or stored only for investigation purposes.

The reporting of use clues by a participant in the sample could be done by sending an electronically signed message to the data collection agency. The purpose of using digital signature is to render the injection of fake members or fake clues more complex and more risky.

The message will contain a number of use clues, with the work identifier and nature of the clue. When the signature does not correspond to the user sending the message, it will be stored for further investigation. When it matches, it will be 
anonymized or stored in a safe store accessible only for later fraud investigation purposes. This requires trust in the data collection agency to truly do so on behalf of the user. This trust will be more readily granted when the user is given the certainty that only data regarding access and upload are transmitted, and when $\mathrm{s} / \mathrm{he}$ has been given the opportunity to review the contents of the transmitted data before sending it.

Sending fake clues will require access to the digital signature or registration as a member. A key element in the prevention of fraud is making sending fake clues as a registered member of the sample a complex and risky act. One possible approach is for the plug-ins to store clue data by encrypting it using a private key kept secret. The user will still be able to decrypt it using a public key to check the contents of the data. Keeping the private key secret will require obfuscation of the $\operatorname{code}^{8}$ for the plug-ins, and sufficiently frequent changes to the key. The compatibility between such a situation and free software licenses such as the GNU General Public License version 3 will need to be studied further. It is possible that similarly efficient schemes exist, that do not require obfuscation.

Finally, it is also important to elaborate on the notion of "making fraud less attractive": artificially downloading or uploading full files (a clue will be generated only when a download is completed) is not free, particularly for uploads. The structure of reported clues will provide important indications of possible fraud, in particular if the number of fake clues necessary for obtaining a motivating reward is sufficiently high. 



\section{Notes}

\section{Introduction}

I. Following a widely adopted practice, we will write "file sharing" rather than "file-sharing" throughout this book, even in contexts such as "file sharing software". See http://en.wikipedia.org/wiki/File_sharing.

\section{The Internet and creativity debate}

I. This expression was used by the French Culture Minister. The French language is more open than English to the figurative use of words.

2. See http://www.lalliance.org.

3. Some of whom had made their own proposals ahead of the Alliance Public Artistes.

4. A special mention is due to Marc Le Glatin's book, Internet: un séisme dans la culture?, published in 2007 (LeGlatin 2007).

5. The reader will find an up-to-date account of the situation in the English-speaking section of La Quadrature du Net's website, see http://www.laquadrature.net/en.

6. Loi n² 2009-669 of 12 June 2009 and Loi n² 2009-I3II of 28 October 2009.

\section{The value of non-market sharing}

I. See section $5 \cdot 3$.

2. Orphan works are copyrighted works whose rights holders are not known or cannot be contacted. It is thus impossible to obtain permission to use them. An impressively large proportion of cultural works are orphan works, in particular for books.

3. See http://en.wikipedia.org/wiki/Napster.

4. FTP sites and the Usenet news groups predated Napster by close to 20 years, and they were widely used to share contents such as photographs or software.

5. Peer-to-peer file sharing is the sharing of files between one person and another using various protocols (technical standards for machine to machine communication) on peer-to-peer networks such as the Internet. A peer-to-peer network is a network where every machine is considered as an emitter as well as a receiver.

6. See http://en.wikipedia.org/wiki/Comparison_of_file_sharing_applications.

7. By now the reader will understand that, when we talk of the recognition of sharing, we mean the attribution of a right to practice it without having to request a specific authorization from the authors or copyright owners.

8. For a more detailed definition of a many-to-all cultural society, see p. 50.

9. In the US, fair use provides someone accused of infringement with a defense against prosecution based on the fact that the activities that took place constitute a fair use of 
the work whose prohibition would damage other values (such as criticism, for instance). Fair dealing is a weaker version in other common law countries such as the UK. In civil law countries, exceptions and limitations to copyright were defined on a case by case basis without a general overarching concept. In international harmonization, exceptions and limitations were used as the federating principle, and the fair use chapter is now under the exception and limitation chapter of copyright in the US Code.

Io. Most copyright infringement cases brought against non-commercial uses by individuals were initiated by collecting societies, large companies or their lobby groups, or financially greedy heirs of deceased artists, rather than by individual authors. The exceptions arose from some big income music groups, whose representation was delegated to managers, or authors that were particularly keen on control. When cases were brought, the outcome was geographically contrasted. In Europe, courts often invoked exceptions or procedural issues to acquit defendants. When defendants were found guilty of infringement but were not deemed to be aiming to make a profit, the sanctions and damages were generally limited (Hugenholtz 2008). In the US, the large number of cases initiated by the Recording Industry Association of America (RIAA) against non-commercial file sharers led to sentences with enormous damages tags (see section 5.2). In the end, this backfired against RIAA, which had to renounce suing such cases in the face of public indignation. These situations led the media industry interest groups and, in Europe, the collecting societies, to request stronger (in comparison to the European practice) and automatic sanctions, bypassing the judiciary by allowing private parties (such as Internet Service Providers or administrative authorities) to set the sanctions, or using legal ordnances to limit the leeway of the judges.

II. See http://www.worldwidewebsize.com/.

12. DVD disks and players were available starting in November 1996, but work on the format started in I993, see http://en.wikipedia.org/wiki/DVD.

13. See (FLOSSIMPACT, 2006, pp. 230-234, Lehman 1995) for the arguments brought forward to justify such provisions.

I4. See http://www.opencontent.org/opl.shtml. This license was discontinued when the Creative Commons licenses became widely used.

I5. See http://www.gnu.org/licenses/fdl.html.

I6. See http://creativecommons.org/licenses/.

17. See http://flickr.com.

I8. See http://www.flickr.com/creativecommons/.

19. There are several files for the same work, but most files contain generally a full album.

20. In April 2008, the ITunes Store claimed to have 6 million song tracks available. See: http://answers.yahoo.com/question/index?qid=200804191307I3AAHHUum.

2I. Since there are many different rights holders for film and TV works, the challenge of identifying rights holders can be severe.

22. See http://openlibrary.org/

23. See http://wikisource.org/.

24. See http://www.gutenberg.org.

25. This conceptualization was suggested by Hervé Le Crosnier. 
26. More precisely I.IO4.

27. Another factor that can impact our analysis is that the unit of music sharing on $\mathrm{P}_{2} \mathrm{P}$ tends to be a full album, while (Page-Garland 2009) studied single track commercial sales. However, the sizes of the two universes are similar, which should limit the impact of the different sharing unit.

28. We are extremely grateful to these researchers for providing us with advance access to this data, which will be published as a paper in English by the time this book is published.

29. See Appendix A and figure A.4 for explanations of the impact of the size of an universe on the share of attention going to a given percentage of works for the same value of the parameter of Zipf's law.

30. See http://en.wikipedia.org/wiki/DC\%2B\%2B.

3I. Here the authors mean "downloading".

32. Major book publishers are presently considering joining the war.

33. We assume here that other factors - such as promotion - are kept equal.

34. Our translation.

35. For the impact on download sales, see (Andersen-Frenz 2008, Marsouin 2008, Oberholzer-Strumpf 2010, Martikainen 2010) and the other studies referenced in section I.2 of the Studies on File Sharing page of La Quadrature du Net site, See http://www. laquadrature.net/wiki/Studies_on_file_sharing_eng.

36. This statement seems to apply to iTunes globally, not to SACEM members, as the total number of titles generating download rights for SACEM was only 844,I86 in 2009.

37. See below page 62 .

\section{Sustainable resources for creative activities}

I. See http://www.senat.fr/leg/pjlo7-405.html, our translation.

2. At the very most $20 \%$.

3. The sale of physical carriers for information will remain - whatever some may say - a significant activity: in a world where information is abundant and perpetually evolving, we need carriers to freeze it in a stable form and store it. However, the turnover of this activity will no doubt decrease compared to the time when information could only be accessed via physical carriers.

4. Public subsidies by all levels of government represent $30-50 \%$ of the financing of cultural activities in developed countries.

5. See in particular point Io of the How-to for Sustainable Creativity of the Free Culture Forum, See http://fcforum.net/sustainable-models-for-creativity/how-to-manual|\#models.

6. The four elements listed may be understood as cumulative.

7. Such as performers, artistic and technical contributors.

\section{Which rights for whom? A choice of models}

I. Michael Goldhaber had already stressed this, explaining the greater value attached to performance by the facts that the audience is "getting illusory attention from the artist". Our work showed that the de facto economic value attached to live performance is 
up to 20 times greater than for DVD and roo times greater than for live broadcast. Whether or not they have the illusion of getting attention from the artist, individuals make a great difference between physical presence and media.

2. At first sight, advertising appears to be paid by announcers, but the cost of it is ultimately transferred to consumers of their products.

3. DVD anti-copy protection, known as content scrambling, was cracked as early as 1999 by the DeCSS software written initially by Jon Lech Johansen, a young Norwegian programmer. DeCSS is widely distributed outside of the US and used world-wide for playing DVDs with free software and recovering the ability to copy them. This software was the object of a judiciary injunction in the US that led to a mobilization of the programming community, with some humorous and artistic by-products. See for instance (Touretzky 2004).

4. One of these cases, Viacom vs. Google, was settled in favor of Google, cf. US Southern New-York DC, 23 June 20I0, see http://www.google.com/press/pdf/msj_decision.pdf.

5. In some countries, such as France, the telecommunications competition regulatory authority (ARCEP) broke the exclusivity of the contract and forced Apple to also offer its products through other operators.

6. Apple iTunes Plus titles are sold without DRM. This means that users who wish will be able to share them without major effort, the legality of this practice depending on whether one remains or not within the realm of home copying laws. However, iTunes software permits an easy sharing between iTunes users possessing Apple devices. With a common configuration of the software, it even occurs automatically when various users connect their devices to the same computer.

7. See page 46 .

8. The model can be extended to advertising-based sites, generally on the basis of the payment of a share of the advertising revenues.

9. Laurent Petitgirard was re-nominated as President of SACEM on I5 June 2011.

Io. In the European legal framework (2001/29/CE directive, art. 3.2), copyright holders, performers and producers of phonograms (phonorecords in the US) and videograms (videorecords in the US) are granted an exclusive right to authorize or not the act of making works available to the public "in such a way that members of the public may access them from a place and at a time individually chosen by them".

II. Loi 95-4 du 3 janvier 1995 and Article L3II-I of the Code la propriété intellectuelle.

I2. The first RIAA lawsuits against individual file sharers were launched on Sept. 8, 2003. The number of these suits culminated in 2005 with close to 6,000 cases, then progressively diminished over the years until RIAA announced at the end of 2008 that it was abandoning this strategy (Kravets 2010).

13. All figures in this paragraph are extracted from the 2010 Statistical abstract of the US Census Bureau; 2007 is the last year for which figures are available.

I4. In France, the turnover of the 4 musical majors strongly decreased due to the shrinking offer of titles, but their profit margins never stopped rising (particularly if measured in terms of profits per album or per title).

I5. The collected sums for 2009 reached $€ 762$ million.

I6. See http://slashdot.org. 
17. "At least in the view of most Americans and Western Europeans, distributive justice requires giving each person in a collective enterprise (whether it be a project, an industry, or a society) a share of its fruits proportional to his or her contribution to the venture" wrote William Fisher in support of compensatory rewards. Important thinkers (Bell 1973, Passet 1979, MoulierBoutang 2010), in both the US and Europe, share the view that among the changes induced by the information revolution, one of the most important is that it is no longer possible to determine the specific contribution of a person to society or even to a precise venture. They defend a definition of distributive justice not as opposed to contributive justice (to each according to his/her contribution) but as complementary (to each according to what creates a better society ... as far as we can know).

I8. Bobbs-Merrill Co. v. Straus, 2Io U.S. 339 (Ig08). Codified in I7 USC § rog.

I9. Epuisement des droits in French. For the European case law, see CJCE, 20 January I98I, Musik-vertrieb vs. GEMA.

20. Due to the predominance of these terms in international treaties, what remains of the first sale doctrine is now encoded in the US Code as a limitation to exclusive rights.

21. I7 USC § 1008 and $\S 1003$.

22. In France, for instance, Loi $n^{\circ} 85-660$ du 3 juillet 1985 .

23. There is debate on whether this will be upheld in further court decisions and if it would stand scrutiny in terms of compatibility with European law, but it stands unrebutted until now.

24. For music, for example, re-distribution is done according to 3 criteria: sales of records, radio broadcast and phone polls of users. All three tend to re-distribute the levies to a set of works which is much less diverse than those actually copied on blank carriers, notwithstanding the fact that these carriers are also used for storing other information such as personal photographs, data or software.

25. Agreement on Trade-Related Aspects of Intellectual Property that was part of the treaty giving birth to the World Trade Organization.

26. The most visible criticism targeted consequences for access to drugs in poor countries.

27. When negotiating a contract that conditions the commercial access of one's works to the public, the balance of power is very unequal. We intentionally leave aside the case of "work for hire" or other situations where copyright is from the start granted to an investor, so that our reasoning applies regardless of whether the reader lives in a country where a copyright tradition exists, or one that still considers itself to have an author rights legislation.

28. 200I $/ 29 / \mathrm{CE}$.

29. COM(2008) 466/3, see http://ec.europa.eu/internal_market/copyright/docs/copyrightinfso/greenpaper_en.pdf.

30. See below on page iI7.

3I. Under the adopted (but still to be ratified in Europe) Anti-Counterfeiting Trade Agreement and the announced European initiatives on IPR enforcement if they follow a previously tabled and withdrawn proposal, this statement could constitute abetting and inciting to infringements. Although these would not be profit-driven, they could nonetheless be deemed to be of a commercial scale, because they allegedly cause 
commercially significant effects. As such, the authors of the offending sentence could be subject to criminal sanctions, including jail.

32. See http://www.creationpublicinternet.fr.

33. See http://www.vgrass.de/? $\mathrm{p}=\mathrm{Ig3}$.

34. See http://www.cptech.org/a2k/pa/ and http://www.tacd-ip.org/blog/the-paris-accord/.

35. See http://fcforum.net/fcf2oro.

\section{Defining rights and obligations}

I. See http://www.keionline.org.

2. Francis Muguet's sudden death in 2009 was a great loss.

3. Music radio broadcasting has very little diversity in France, due to the dominant position of a few radio networks.

4. Transposition in French law of the 2001/20/CE European Directive on Copyright and Neighbouring Rights in the Information Society.

5. In particular the aforementioned Pour le cinéma group, see http://pourlecinema.overblog.frl.

6. Loi n²009-669 du I2 juin 2009 favorisant la diffusion et la protection de la création sur internet.

7. Three warnings of infringement, and you are out of the Internet.

8. The benefit of the financial reward is optional.

9. Council Directive 93/83/EEC. Whereas 28 reads: Whereas, in order to ensure that the smooth operation of contractual arrangements is not called into question by the intervention of outsiders holding rights in individual parts of the programme, provision should be made, through the obligation to have recourse to a collecting society, for the exclusive collective exercise of the authorization right to the extent that this is required by the special features of cable retransmission; whereas the authorization right as such remains intact and only the exercise of this right is regulated to some extent, so that the right to authorize a cable retransmission can still be assigned; whereas this Directive does not affect the exercise of moral rights. The last sentence is an interesting example of Newspeak: it should read "in practice, the exercise of moral rights by authors will be made de facto impossible beyond the simple refusal to have one's work transmitted by satellite (or even satellite and broadcast in the case of a simultaneous transmission)".

Io. A debate exists within the Creative Commons communities on how to handle moral rights in CC licenses that authorize modifications. The present licenses for the French jurisdiction do not waive moral rights: they can't according to the theoretical inalienability of moral rights. This leads to a degree of legal uncertainty for the user (if he or she intends to create a derivative work). But this uncertainty is limited, provided the user respects a few ground rules, as the practical implementation of moral rights in courts pays much attention to the original intention of authors, and this intention is not ambiguous: an author who authorizes modifications to a work ... knows the work can be modified.

II. See chapter Io.

I2. It could be a useful complement to the Creative Contribution, see page 64 . 
I3. Part of this paragraph was published earlier on the author's blog, see http://paigrain. debatpublic.net/?p=1629\&lp_lang_view=en.

I4. Documents that were never the object of copyright or whose copyright is extinct.

\section{How much?}

I. This particular distinction is essential, because it influences the risk of a new round of "regulatory takings" claims. See above page 76 and below page II7.

2. See http://www.laboiteverte.fr/.

3. Our translation.

4. See blublu.org, http://www.davidellis.org/ and http://vimeo.com/6555I6I (License CCBy-NC-ND).

5. Depending on the context, we use the terms creators, contributors or authors and other contributors interchangeably to designate the producers of digital works that are freely shared. We consider performers (in music, theater plays, dance or movies) to deserve the name of creator on equal standing with authors or directors. More generally, we do not put any special emphasis on the word creator.

6. The notion of an album will survive even if records disappear - which we do not believe will happen - because it defines a listening unit, one particular form of a playlist.

7. (Stiglitz 2009), our translation.

8. See http://magnatune.com/, http://www.jamendo.com/ and http://pragmazic.net/bin/ accueil.php?lang=en.

9. If a non-proportional reward function (see below page 94) is put in place, one will need to consider how the coexistence of albums and tracks as work units might bias the system.

Io. Weighted by their relative contribution to each work in the case of works with several contributors.

II. If one excludes royalties for neighboring rights of performing musicians, which already come from mutualized financing schemes.

I2. In France, in 2003, only 8,700 authors and performers for all media received enough royalties to induce them to pay contributions towards retirement benefits on the basis of these royalties (Benhamou-Sagot-Duvauroux 2007).

13. Refer to the box on currencies on page 63 for explanations regarding how we deal with currency equivalents in this book.

I4. In developed countries.

I5. Or more than $2 \%$ of the total adult population. Source: 20 Io Statistical Yearbook of the US Census Bureau, table in23.

I6. In other countries, a possible minimum would be for 0.8 to $1.0 \%$ of persons with broadband Internet access to receive the purchasing power standard equivalent of $\$ 200$.

17. We come back to this issue when discussing the question of whether we are "monetizing the non-market" in FAQ is on page 167.

I8. The number of broadband Internet subscribing households in each country was taken from http://en.wikipedia.org/wiki/List_of_countries_by_number_of_broadband_Inter 
net_users. The total number of subscribers for 2009 corresponds approximately to the number of home subscribers at the beginning of 20Ir. For details on the implementation of the model in each case, see appendix B.

19. Zipf laws of parameter I.0, 0.9 and 0.8 .

20. According to the Wikipedia page: "Artists and repertoire (A\&R) is the division of a record label that is responsible for talent scouting and overseeing the artistic development of recording artists. It also acts as a liaison between artists and the record label.”, see http://en.wikipedia.org/wiki/Artists_and_repertoire. Independent labels are responsible for most of the true A\&R activities, major labels intervening mostly at a later stage, to promote already known artists more widely. In this book, we consider $A \& R$ to be at the core of the long-term role of publishers in Internet culture. Yet in recent years, major labels have made major cuts in their A\&R budgets, to make room for more promotion expenses for established artists.

2I. See http://www.kickstarter.com/help/faq|\#SoThisIsnAbouInve.

22. See http://www.mymajorcompany.com/Artistes/gregoire.

23. See https://www.sellaband.com/.

24. See http://www.touscoprod.com/.

25. The US Bureau of Labor Statistics reports that, in 2009, the total of wages for 27-0000 Arts, Design, Entertainment, Sports, and Media Occupations category within the wider Industry NAICS Newspaper, periodical and directory was close to $\$ 8,000$ million. Subtracting the book publishing sector (which we have accounted for already) and adding externalized content production costs (such as syndicated works) would lead to a gross estimate of $\$ 7,000$ million.

26. Source: 2010 Statistical abstract, US Census Bureau, tables IIgI.

27. We discuss this view in chapter II.

28. Our global estimates include public grants. The lower per capita investment is mostly due to a weakness of private investment in some sectors.

29. Cf. http://en.wikipedia.org/wiki/Flickr and http://www.flickr.com/creativecommons/. The figure for Creative Commons photos is from March $201 \mathrm{I}$ while the total figure is from October 2009.

30. See http://www.bls.gov/oco/ocos264.htm.

31. According to (Oberholzer-Strumpf 2010), the vast majority of Internet file sharing traffic in data volume was already composed of video files in 2006.

32. (Oberholzer-Strumpf 2010) quotes (Ferguson 2006) as showing "that eDonkey traffic levels were largely unaffected in 2006 when legal authorities forced closure of a large network of servers".

33. These three hypotheses are those described above, used to produce the figures quoted in table 7.I, for the US.

34. Assuming that a quarter of all rewards would go to music.

35. The authors justify not analyzing the years after 2004 by a change in the coverage of venues by Pollstar from 2005 (to include a much wider range of small venues). It would have been interesting to get some insight into what happens in this new situation.

36. Between 1995 and 2002, data being unavailable for 2003 and 2004.

37. US Census Bureau 2010 Statistical Abstract, table IIo2. 
38. See The Numbers, http://www.the-numbers.com/

39. Syndicat de l'édition vidéo.

40. See http://www.sevn.fr/?page_id=6.

4I. Source: Centre National de la Cinématographie, Statistiques sur la production cinématographique en France, 2010 data for 2009, "Investissements dans la production française”, see http://www.cnc.fr/CNC_GALLERY_CONTENT/DOCUMENTS/statistiques/ par_secteur_FR_pdf/ProductionCine.pdf.

42. Décret no 200I-I329 du 28 décembre 200I, http://www.legifrance.gouv.fr/affichTexte. do?cidTexte=JORFTEXToooooo $77060 \mathrm{r}$.

43. The box office is measured jointly for US and Canada.

44. Sources: The Numbers, Nash Information Services, http://www.the-numbers.com/mar ket/. See also the Major film studios entry of Wikipedia, http://en.wikipedia.org/wiki/ Major_film_studio|\#Today.27s_Big_Six.

45. To some degree: books can be photocopied, but with a significant loss of usability.

46. Source: http://www.bowker.com/index.php/press-releases-2007/I46 and (Jones 2009). Note that according to other methodologies, there are only between 170,000 to 200,000 books every year, but with similar trends.

47. See http://www.thebookseller.com/news/8589I-self-publishing-takes-the-lead-in-us. html.

48. Source: Livres Hebdo - Électre biblio/DEPS, http://www.lemotif.fr/fr/le-livre-en-ile-defrance/chiffres-cles/bdd/article/49.

49. Source: http://www.sevn.fr/?page_id=6.

50. US Census Bureau 2010 Statistical Abstract, table rog6. The figures quoted in this table, whose source is Mediamark Research Inc., should be interpreted with some caution, as they are difficult to reconcile with table II20, originating from the same source.

5I. See http://www.broadband.gov/plan/

52. See for instance article 14 of the 1996 Phonogram Treaty and its important limitation by article I2.2, see http://www.wipo.int/treaties/en/ip/wppt/trtdocs_wo034.html/\#PIII_ $\underline{13298}$.

53. "Technical protection measures" is a legal term. In media business contexts, TPM are also called DRM, for Digital Rights Management, so as to disguise their true nature as access and usage control devices.

54. The fate of this law proposal is uncertain at the time of writing, further to the nomination of a new Minister for Culture.

55. See above page 87 .

56. The droit de retrait and droit de repentir exist only in France, Italy and Greece. They allow the author to request the withdrawal of material that has already been divulged, in exchange for a monetary compensation of the costs associated with this decision. They are extremely rarely applied in practice. When they have been, it was usually for dubious purposes, such as when Céline's heirs requested the withdrawal of the antisemitic passages of his works.

57. Including media spending, attending cultural events and amateur artistic activities, but excluding digital equipment.

58. US Census Bureau 20Io Statistical Abstract, table II95. 
59. They note there was a strong growth in leisure services spending and a smaller decrease in leisure goods spending. This increase in services and decrease in goods is noted in all fields impacted by information and communication technology.

6o. Simply because households without a broadband connection are generally poorer or live in rural areas.

61. See (ARCEP 2008): in 2007, the monthly expenditure was $€ 55$ on average for all households and $€ 72$ for equipped households. As of 2010, almost all households have become equipped.

62. Source: Bureau of Labor Statistics Consumer Expenditure Survey, http://www.bls.gov/ cex/cellphones2007.htm.

63. See for instance 2010 Statistical yearbook of the US Census Bureau, table II20.

\section{Sustainable financing for the commons}

I. See the Knowledge Ecology International page on the WHO Negotiations on Public Health, Innovation and Intellectual property, http://www.keionline.org/whoiplusa and the funding of AIDS prevention and treatment by the first global tax on airborne passenger transport.

2. The number of households is growing, due to the increase in single-person or small households. However this also means that the average resources per household grow much more slowly or even decrease, for instance for single-parent families.

3. Thus, a total amount of $5 \cdot 5-7.7 \%$ of the investment in the production of an initial copy of works in all covered media.

4. Even in comparison to the best first instance case law in Spain, the scope of legal sharing will be clarified and extended.

5. See http://www.vgrass.de/? $\mathrm{p}=\mathrm{I} 93$. Brazil is a specific case because there is a wider success of voluntary sharing in media such as music. See FAQ entry I6 in chapter II for an example.

6. We note that this leads to non-negligible economic development in country A. Given the importance of a head-start in Internet-based activities, this is likely to be sustained.

7. See for instance New Zealand 1994 copyright act $n^{\circ}$ I43, art. 226, http://www.legisla tion.govt.nz/act/public/I994/or43/latest/DLM346899.html, which explicitly excludes geographical zoning from protection against circumvention.

8. Except if country B blocks all IP addresses based in country A, an option which defies common sense but will certainly be considered by some interest groups in country B.

9. See chapter Io.

Iо. See http://www.plos.org.

II. For the former, see the Svalbard Global Seed Vault project where seeds are stored in a vault in the permanently frozen ground of an island of the Spitsbergen archipelago. For the second mode, see (Saunier-Meganck 1995) and the farmer's seeds networks coordinated by the journal GRAIN.

I2. Because they required genetic homogeneity of seeds. 


\section{Organization and complementary policy measures}

I. See http://www.laquadrature.net/en/Net_neutrality.

2. See above on page 62 .

\section{Usage measurement for equitable rewards}

I. Bernard Miyet, CEO of SACEM, the collecting society for music authors and composers in France, expressed this point of view in a radio debate in which we participated.

2. Declarations of Eduardo "Teddy" Bautista, president of SGAE, the Spanish collecting society for music composers, authors and publishers, and Pascal Rogard, CEO of the SACD collecting society for audiovisual and theater authors in France, in public debates with us.

3. Laurent Petitgirard, former president of SACEM, in a debate with us.

4. For statutory licensing to educational public broadcasting in the US, there are 8 classes of radio stations (CopyrightRoyaltyBoard 2007).

5. See http://www.wordpress.org.

6. Unemployment benefit for intermittent workers in the performing arts.

7. They are of course intrinsically unable to distinguish between a legitimate use of a work and an illicit one.

8. See http://www.shazam.com.

9. See http://en.wikipedia.org/wike/Berne_Convention_Implementation_Act_of_ig88

Io. Meaning that the registrant is assumed to be entitled to register the work. This must not be confused with another meaning of copyright entitlement that designates the ability to enforce copyright without having to register the work.

II. See http://www.wipo.int/treaties/en/ip/berne/trtdocs_wooor.html.

I2. International Standard Book Number.

13. These patents can be challenged in Europe as informational patents whose validity is not recognized in the Munich convention. Their validity could also be contested in the US under recent case law regarding informational patents used in business methods. Prior arts challenges could also be possible, as they are generally with any information or software patent.

I4. This must be done in a manner that does not prevent some modification of works for adaptation to formats or dissemination channels, or derivative works when they are authorized.

I5. Some may be surprised that we use free/open source software, that is software that users are authorized to modify, for a process that must be fraud-resistant. It is indeed contrary to free software licenses to prevent users to modify it. However, it is perfectly OK for the data collection agency to ignore data when it has indications that the critical parts of the reporting plug-in have been tampered with, and to make sure that users cannot make such modifications without the data collection agency having a good chance of knowing about it when the data is reported.

I6. Noank Media was founded to implement the measurement systems described in (Fisher 2004). It does not appear to be active anymore.

17. See appendix $\mathrm{C}$ for the justification of these claims. 
I8. The vast majority of French blogs are teenager blogs associated to the Skyblog site, some of which of course have an original design or contents, but many are strongly stereotyped. The source for the first 2 numbers is (Giazzi 2008). The number of the "creative effort" blogs is our best guess: it does not matter for our purpose here if we are mistaken by a factor of 2 . By creative effort, we mean any form of personal originality in form, contents or ideas being expressed or facts being reported.

19. This is 50 times and not 100 times, because, in our model for the reporting by the voluntary sample, we made the hypothesis that the members of the voluntary sample would account for twice as many blog readings per person as the general population.

20. See http://www.kickstarter.com/help/faq.

\section{Clarification and counter-arguments}

I. For a better knowledge of the specificity of software and databases, it is useful to remind oneself of how it entered in the realm of copyright. In the US, software and databases became copyrightable after work by the CONTU Committee in the I970s (made available on the Web by (Hollaar 2003). In Europe, similar debates preceded the adoption of the Munich European Patent Convention of 1972, which precluded computer programs from being patentable inventions. During the debates, it was recognized that software and databases differed from other entities covered by copyright, and some recommended that they remain in the public domain.

2. Directive $96 / 9 / \mathrm{EC}$.

3. See http://creativecommons.org/licenses/by-nd/3.o/legalcode.

4. See the remarkable Gramophone record page of Wikipedia, http://en.wikipedia.org/ wiki/Gramophone_record.

5. The 2010 Brazilian copyright law proposal includes sanctions for music labels paying radio to play particular music tracks (Colombo 20I0).

6. This list corresponds to risks identified in the Lambrinidis report (Lambrinidis 2009), which was adopted by the European Parliament in March 2009; in the French Conseil constitutionnel Décision n 2009-580 DC of Io June 2009 on the first HADOPI law, which foresaw the implementation of the infamous "three-strikes and you are out of the Internet" policy in France (Conseil Constitutionel 2009); and in the opinion formulated by the European Data Protection Supervisor on the negotiations of the ACTA treaty (Hustinx 2010).

7. Cf. intervention by Cay Wesnigk, member of VG Bild-Kunst, in the European Parliament event reported by (Cronin 2010).

8. See http://www.believedigital.com/.

9. See http://flattr.com/.

Io. Censal voting is a system in which only richer people are allowed to vote, or have more votes.

II. See http://www.lalliance.org and, for STIM, (OMahony 2008). 


\section{From proposal to reality}

I. The International Federation of the Phonographic Industry and the Motion Picture Association deserve a special mention. The latter conveniently deleted "of America" from its name.

2. See http://www.keionline.org.

3. See http://www.cptech.org/a2k/pa/.

4. See http://firstmonday.org/htbin/cgiwrap/bin/ojs/index.php/fm/issue/view/rg7 and http://firstmonday.org/htbin/cgiwrap/bin/ojs/index.php/fm/issue/view/200.

5. See above on page 34 .

6. Although with a wider scope than just entertainment.

7. See http://www.kickstarter.com, http://www.yooook.net.

8. See the presentation video at http://flattr.com/register.

9. See http://2010.fcforum.net/en.

Io. See http://fcforum.net/sustainable-models-for-creativity/how-to-manual.

II. See http://news.cnet.com/830I-30685-3-20000092-264.html.

I2. See http://crenk.com/the-pirate-bay-torrent-search-engine-to-be-censored-in-italy/.

I3. See http://creationpublicinternet.fr/blog/index.php?category/Ce-que-nous-proposons.

I4. Statement by Ruth Hyeronimi in the "Future of Intellectual Property" Conference organized by the Goethe Institut and the Committee of Regions of the European Union in Brussels, 23-24 April 2009.

I5. In a 2005 interview (Kanellos 2005), Bill Gates declared that "No, I'd say that of the world's economies, there's more that believe in intellectual property today than ever. There are fewer communists in the world today than there were. There are some new modern-day sort of communists who want to get rid of the incentive for musicians and movie makers and software makers under various guises. They don't think that those incentives should exist."

I6. After a change of management, ADAMI later opposed such proposals.

17. See http://wikis.fu-berlin.de/display/fcrc/Home.

\section{A. Diversity of attention for beginners}

I. See http://en.wikipedia.org/wiki/Zipf\%27s\_law.

2. A probability density function is a mathematical function which one integrates over a specific interval to obtain the probability that the quantity of interest lies in that interval: $\mathrm{P}[\mathrm{a} \leq \mathrm{X}<\mathrm{b}]=\int_{\mathrm{a}}^{\mathrm{b}} \mathrm{f}(\mathrm{x}) \mathrm{dx}$. Integrating from a particular value to infinity gives the probability that the quantity of interest exceeds that value, known as the cumulative probability distribution: $\mathrm{P}[\mathrm{X}>\mathrm{x}]=\int_{\mathrm{x}}^{\infty} \mathrm{f}\left(\mathrm{x}^{\prime}\right) \mathrm{dx}$ '. Conversely, the probability density function can be obtained by differentiating the cumulative probability distribution.

3. Vectorial text indexing for retrieval uses this property by giving a stronger weight to rare words when indexing a text.

4. This normalization is needed because the finite sum of a power law depends on its index.

5. This means that a Pareto's law of parameter I and a Zipf law of parameter I are actually "the same", but this is not true at all for parameters other than I. For instance a 
Zipf law with parameter 0.5 corresponds to a power law of index 3 , and thus to a Pareto's law of parameter 2.

6. Our method is based on directly optimizing the goodness of fit measured by a Kolmogoroff-Smirnoff distance on cumulated distributions. The source code for the corresponding algorithm is distributed under the GNU GPL version 3 license (http://www. $\underline{\text { fsf.org/licensing/licenses/gpl.html) on the book's Web site at : http://www.sharing-the }}$ book.net.

7. (Goldstein et al. 2004) propose a Maximum Likelihood Estimation method, where the resulting goodness of fit is also measured by a Kolmogoroff-Smirnov distance on cumulated distributions, with tables specifically adapted to their method of estimation.

8. Private communication at the First Monday Ioth anniversary Conference on Openness: Code, science and content, Making collaborative creativity sustainable, Chicago, I5-17 May 2006.

9. Works for which the rights holders cannot be identified or reached.

Io. Files named SourceSearch.xml in the data set.

\section{B. The total cost of rewards and their distribution}

I. If $c_{i}$ did not contribute to $w_{j}$ at all, contrib $\left(c_{i, w_{j}}\right)=0$.

2. In figure 7.2 , the size of the universe is kept constant, but in practice, it will vary: if the total cost of the reward is fixed, spreading it more diversely (but still according to a Zipf law) implies an enlarged set of rewarded creators.

3. They can be run interactively or downloaded at http://www.sharing-thebook.org.

\section{Modeling usage measurement}

I. If a better knowledge of the distribution of titles and shared per creator existed, one could do a more rigorous modeling by convoluting this distribution with the popularity distribution.

2. Possibly its variant for variables following power laws with infinite variance.

3. Source: Wikipedia iTunes Store page, http://en.wikipedia.org/wiki/ITunes_Store, quoting Apple Inc. press releases.

4. Based on 3,600 million sales in a year for an average price of $\$ \mathrm{I}$, of which $13 \%$ total are redistributed to author, composers, performers and other contributors, or their assignees.

5. See table 6 in (Veenhof 2009), http://www.statcan.gc.ca/pub/56fooo4m/2006or $/$ tbl/ tblo6-eng.htm.

6. Strictly speaking, we should also take it into account when computing N', but this has little impact. In reality, the most appreciated blogs stand a higher chance of being registered, but we will also ignore this fact for sake of simplicity.

7. By readership per year we mean number of blog content page views, with the usual exclusions of own access, robot access and RSS access without full content display.

8. See http://en.wikipedia.org/wiki/Obfuscated_code. 


\section{Bibliography}

Lada A. Adamic. Zipf, power-laws, and Pareto a ranking tutorial, 2002. Accessible at http:// www.hpl.hp.com/research/idl/papers/ranking/ranking.html.

AFP. La CAF relativise l'impact financier des fraudes aux allocations sociales. Le Monde, 29 April 2010.

Frédéric Aidouni, Mathieu Latapy, and Clémence Magnien. Ten weeks in the life of an eDonkey server. http://arxiv.org/abs/0809.3415, also presented at HotP2P 2009, May 2009, Roma, 2008.

Philippe Aigrain. Macro-economics and time issues in consumer access to media and new media, November i996. unpublished.

Philippe Aigrain. Attention, media, value and economics. First Monday, 2, September 1997. http://firstmonday.org/htbin/cgiwrap/bin/ojs/index.php/fm/article/view/549/470.

Philippe Aigrain. Cause commune: l'information entre bien commun et propriété. Fayard, Paris, 2005. ISBN 2-212-62305-8.

Philippe Aigrain. Diversity, attention and symmetry in a many-to-many information society. First Monday, Io, June 2006. http://firstmonday.org/htbin/cgiwrap/bin/ojs/index.php/fm/article/view/1337/I257.

Philippe Aigrain. Internet \& Création: comment reconnaitre les échanges sur internet en financant la création. InLibroVeritas, Cergy-Pontoise, France, 2008. ISBN 978-2-35209-177-6.

Philippe Aigrain and Raphaël Badin. Attention diversity in eDonkey $\mathrm{P}_{2} \mathrm{P}$. Communication at the 2nd Free Culture Reseach Workshop, http://cyber.law.harvard.edu/fcrw/sites/fcrw/images/Ai grain_, 23 October 2009.

Birgitte Andersen and Marion Frenz. The impact of music downloads and p2p file-sharing on the purchase of music: A study for industry Canada. http://www.ic.gc.ca/eic/site/ippd-dppi.nsf/ eng/h_ipor456.html, 2008. Study of 2006-2007 data.

Chris Anderson. 'The long tail'. Wired, October 2004. http://www.wired.com/wired/archive/ I2.Io/tail.html.

Chris Anderson. The Long Tail, Why the Future of Business is Selling Less of More. Hyperion, New York, 2006. ISBN I-4013-0237-8.

Chris Anderson. Netflix data shows shifting demand down the Long Tail. http://www.longtail. com/the_long_tail/2009/og/netflix-data-shows-shifting-demand-down-the-long-tail. html, September 242009.

Nate Anderson. 'The RIAA? Amateurs. Here's how you sue 14,000+ p2p users'. Ars Technica, http://arstechnica.com/tech-policy/news/2010/06/the-riaa-amateurs-heres-how-yousue-p2p-users.ars, 2010.

ARCEP. Les ménages français dépensent 93 euros par mois en services de télécommunications. Lettre de l'Autorité de Régulation des Communications Electroniques et des Postes, http://www.arcep.fr/uploads/tx_gspublication/lettre59.pdf, January-February 2008. 
Alliance Public Artistes. Public et artistes pour une solution légale aux échanges sur internet. http:// www.lalliance.org, 2005.

Philippe Axel. Entretien avec le Président de la SACEM. http://www.philaxel.com/2005/0I/3I/ blog-musical/entretien-avec-le-president-de-la-sacem/, January 2005.

Maya Bacache, Marc Bourreau, Michel Gensollen, and François Moreau. Les musiciens dans la révolution numérique - inquiétude et enthousiasme. http://ses.telecom-paristech.fr/bourreau/ Recherche/LivreAMImanuscriptı2octobre2009.pdf, October 2009. Telecom ParisTechCNAM-ADAMI-IRMA.

Stewart Baker. When DHS questioned ACTA. http://volokh.com/20II/04/27/when-dhs-ques tioned-acta/, 27 April 201 .

Bodó Balázs and Zoltán Lakatos. 'A filmek online feketepiaca és a moziforgalmazájs: I. rézs: A trasakciózintû elemzés lehetôsegei’. Szociológiai Szemle, 20(3), 34-75, 2010. http://www.warsystems.hu/publikaciok/online-black-market-of-films-and-cinematicdistribution-in-hungary/

Marta Beck-Domzalska. Cultural statistics pocketbook. http://epp.eurostat.ec.europa.eu/cache/ ITY_OFFPUB/KS-77-07-296/EN/KS-77-07-296-EN.PDF, November 2007.

Daniel Bell. 'The coming of Post-Industrial Society: A Venture in Social Forecasting'. Basic Books, I973. ISBN 0-465-0I28I-7. Republished in 1999 with a new preface by the author.

Françoise Benhamou and Dominique Sagot-Duvauroux. Place et rôle de la propriété littéraire et artistique dans le fonctionnement économique des filières d'industrie culturelle. http://www2.cul ture.gouv.fr/deps/pdf/etudes/CE_2007-8_synthese_BAT05I207.pdf, December 2007.

Yochai Benkler. The Wealth of Networks: How Social Production Transforms Markets and Freedom. Yale University Press, 2006. ISBN 9780300110562.

Ann M. Blair. Too Much to Know: Managing Scholarly Information before the Modern Age. Yale University Press, November 2010. ISBN 9780300II25II.

Bruno Blasselle. Histoire du Livre. Gallimard Découvertes, Paris, December 2008. ISBN 9782070122479 .

David Bollier. 'Online collaboration goes legit: A new Vermont law lays the foundation for new types of virtual corporations'. On the Commons, http://onthecommons.org/onlinecollaboration-goes-legit, I6 December 2008.

Marc Bourreau and Michel Gensollen. 'L'impact du numérique sur la filière de la musique enregistrée'. In Pierre Vialle, editor, Mutation des STIC : acteurs, resources et activités, pages I4II92. Lavoisier, Paris, 2007.

James Boyle. The second enclosure movement and the construction of the public domain, 2003. Available at : http://james-boyle.com/.

James Boyle. The Public Domain: Enclosing the Commons of the Mind. Yale University Press, 2008. ISBN 978-0300137408.

Peter Brantley. The orphan monopoly. http://blogs.lib.berkeley.edu/shimenawa.php/2009/ 03/I5/the-orphan-monopoly, March 2009.

Erik Brynjolsson, Jeffrey Yu, and Duncan Simester. Goodbye Pareto principle, Hello Long Tail: The effect of search costs on the concentration of product sales, 2006.

http://ssrn.com/abstract953587.

Bart Cammaerts and Bingchun Meng. Creative destruction and copyright protection - regulatory responses to file-sharing. London School of Economics and Political Science, http://www. 
scribd.com/doc/51217629/LSE-MPPbriefi-creative-destruction-and-copyrightprotection, March $200 \mathrm{I}$.

Centre National de la Cinématographie. Evolution du public des salles de cinéma 1993-2009. http://www.cnc.fr/Site/Template/Tr.aspx?SELECTID=3972\&ID=289I\&Type=0\&Annee $=0 \& \mathrm{t}=3$, juillet 2010 .

ChillingEffects. Frequently asked questions (and answers) about anticircumvention (dmca). Samuelson Law, Technology \& Public Policy Clinic, University of California, Berkeley http:// www.chillingeffects.org/anticircumvention/faq.cgi.

Collective. Declaration: A balanced interpretation of the three-step test in copyright law. http://www.ip. mpg.de/shared/data/pdf/declaration_three_steps.pdf,2008.

Manuela Correia Botelho Colombo. 'Brazil's discussion on copyright law reform - response to the digital era?' IP Watch, http://www.ip-watch.org/weblog/2010/07/I5/brazil $\% \mathrm{E}_{2} \% 80 \% 99$ s-discussion-on-copyright-law-reform-response-to-the-digital-era/, I5 July 2010.

Conseil Constitutionnel. Décision 2009-580 dc du 10 juin 2009 sur la Loi favorisant la diffusion et la protection de la création sur internet. http://www.conseil-constitutionnel.fr/conseil-constitu tionnel/francais/les-decisions/acces-par-date/decisions-depuis-1959/2009/2009-580-dc/ decision-n-2009-580-dc-du-Io-juin-2009.42666.html, Io June 2009.

CONTU. Final report of the national commission on new technology uses of copyrighted works. Digitized and made available on-line under the direction of Lee A. Hollaar at http://digitallaw-online.info/CONTU/, September 2003.

Copyright Royalty Board, Library of Congress. 'Noncommercial educational broadcasting statutory license'. The Federal Register, 72(230), 30 November 2007. http://regulations. justia.com/view/96134/.

Antonella Corsani and Maurizio Lazzarato. Intermittents et précaires. Editions Amsterdam, 2008. ISBN 978-2-35480-02I-5. http://www.cip-idf.org/IMG/pdf/Intermittent-Ultimateinterior-file.pdf.

David Cronin. New business models proposed in debate on EU culture and copyright. http://www.ipwatch.org/weblog/2010/06/og/new-business-models-proposed-in-debate-on-eu-cul ture-and-copyright/, 9 June 2010.

Robert Darnton. A digital library better than google+. New-York Times, 23 March 20Ir. http:// www.nytimes.com/2011/03/24/opinion/24darnton.html.

Mathias Daval. 'Etude sur l'offre numérique illégale des livres français sur internet en 2009'. Edysseus Consulting, http://www.lemotif.fr/fichier/motif_fichier/72/fichier_fichier_ etude_ebookz.pdf, October 2009.

Juan-Carlos de Martin. 'La legge che zittirà i blogger'. La Stampa, 28 July 2010. http://www. lastampa.it/_web/cmstp/tmplRubriche/editoriali/gEditoriali.asp?ID_blog=25\&ID_artico $\mathrm{lo}=7647$.

Nicolas Dehorter. Les acteurs du financement participatif sur Internet. http://musique.suiteror.fr/ article.cfm/les-acteurs-du-financement-participatif-sur-internet, July 2gth 2010.

Sylvain Dejean, Thierry Pénard, and Raphael Suire. Une étude sur les pratiques de consommation de vidéos sur internet. http://www.marsouin.org/IMG/pdf/etudeusagep2p.pdf, December 2008. 
Giulio D'Eramo. 'Italy's media unite in protest against latest 'gag law'. The Latest.com, http://www.the-latest.com/italys-media-unite-protest-against-new-gag-law, 24 August 2010.

Valérie Deroin. 'Diffusion et utilisation des TIC en France et en Europe en 2009'. DEPS, Ministère de la culture, http://www.culture.gouv.fr/deps, 2010.

Roberto di Cosmo. Création artistique libre dans un internet libre. http://www.dicosmo.org/ MyOpinions/index.php/20Ir/or/27/Iog-manifesto-creation-et-internet-libre, 25 January 2011.

Cultura Digital. 'Carta de representantes da sociedade civil á Presidente Dilma Roussef e à Ministra da Cultura Ana Buarque de Hollanda'. Cultura Digital, http://culturadigital.br/ cartaaberta/, 28 December 2010. English translation as Open Letter by the Brazilian civil society to President-elect Roussef and Minister of Culture Ana Buarque de Hollanda by Volker Grassmuck, http://www.vgrass.de/? $\mathrm{p}=638$.

Cory Doctorow. Down and Out in the Magic Kingdom. Tor Books, USA, February 2003. ISBN 0-7653-0436-8.

Cory Doctorow. For the Win. Tor Books Teen, USA, May 2010a. ISBN 978-0765322166.

Cory Doctorow. 'Brazil's copyright law forbids using drm to block fair use'. Boing-boing, http://boingboing.net/2010/07/ro/brazils-copyright-la.html, ro July 20rob.

Olivier Donnat. Pratiques culturelles et usages d'internet. http://www2.culture.gouv.fr/deps/fr/pra tiquesinternet.pdf, November 2007.

Amir Efraty and Jeffrey A. Trachtenberg. Judge rejects google books settlement. Wall Street Journal, 23 March 201 .

http://online.wsj.com/article/SBIooor424052748704461304576216923562033348.html.

Envisional. 'An estimate of infringing use of the internet'. Technical report, http://documents. envisional.com/docs/Envisional-Internet_Usage-Jan2oIr.pdf, January $201 \mathrm{I}$.

FCC. 'Sixth broadband deployment report'. Federal Communications Commission (FCC), http:/l www.fcc.gov/Daily_Releases/Daily_Business/2010/dbo720/FCC-I0-I29AI.pdf, July 2010.

David Ferguson. Trends and statistics in Peer-to-Peer. Presented at the Workshop on Technical and Legal Aspects of Peer-to-Peer Television, 2006.

Pascale Ferran and al. 'Le milieu n'est plus un pont mais une faille'. Stock, 2008. Rapport du Club des 13, ISBN 978-2-234-06132-3.

Willian W. Fisher. Promises to Keep: Technology, Law and the Future of Entertainment. Stanford University Press, Stanford, CA, USA, 2004.

Andréa Fradin. 'Dans ce rapport, ce qui saute aux yeux, c'est l'incompétence'. Interview of Benjamin Bayart in Libération/Ecrans, 13 August 2010.

Rishab A. Ghosh. 'Economics is dead, long live economics! A commentary on Michael Goldhaber's The attention economy'. First Monday, 2, May 1997. http://www.uic.edu/ htbin/cgiwrap/bin/ojs/index.php/fm/article/view/529/450.

Rishab A. Ghosh. 'Cooking pot markets: an economic model for the trade in free goods and services on the internet'. First Monday, 3, March 1998. First published in 1996 on the Web. Also published in the Brazilian Journal of Economics.

Shubha Ghosh, Michael A. Gollin, Dean A. Monco, Adam Mossoff, and Loren A. Smith. Are patents "private property" under the fifth amendment? A debate at the Federalist society for law and public policy studies, http://www.fed-soc.org/publications/pubID.I6I/pub_detail.asp,

March 2007. 
Danièle Giazzi. 'Les médias et le numérique'. Report of the author to the French Secretary of State to the Digital Economy, http://danielegiazzi.typepad.fr/ump/files/septembre 2008rapport_giazzicomplet.pdf, September 2008.

Marc Le Glatin. Internet : Un séisme dans la culture? Editions de l'attribut, Toulouse, 2007.

Michael Goldhaber. 'The attention economy and the net'. First Monday, 2, April I997. http:// www.uic.edu/htbin/cgiwrap/bin/ojs/index.php/fm/article/view/537/458.

Michel L. Goldstein, Steven A. Morris, and Gary G. Yen. 'Problems with fitting to the power-law distribution'. The European Physical Journal B, 4I(2), September 2004. preprint from http://arxiv.org/abs/cond-mat/0402322.

'Groupe Civilisation numérique du Laboratoire des Idées du Parti Socialiste'. La société créative pour tous : une contribution pour un nouveau modèle de développement. http:// laboratoiredesidees.parti-socialiste.fr/index.php/2010/04/une-societe-creativepour-tous/?utm_source=rss\&utm_medium=rss\&utm_campaign=une-societe-creativepour-tous, April 2010.

Nicolas Guillaume. 'La licence globale resurgit quand la hadopi prend ses marques'. ITEspresso, http://www.itespresso.fr/la-licence-globale-resurgit-quand-la-hadopi-prend-sesmarques-34949.html, 5 May 2010.

Steve Hillage and commentators. Spotify's income for artists is microscopic - this model cannot work. http://getsatisfaction.com/spotify/topics/spotifys_income_for_artists_is_ microscopic_this_model_cannot_work, November 2009.

Bernt Hugenholtz. Introductory keynote discussion to the $3^{\text {rd }}$ communia workshop. Report at http://www.communia-project.eu/node/165, October 2008.

Geoffrey P. Hull. The recording industry. Routledge, 2004. ISBN 978 04I5968034. First published in 1998.

Peter Hustinx. 'Second opinion of the European data protection supervisor on the review of directive 2002/58/ec conccerning the processing of personal data and the protection of privacy in the clectronic communications sector'. Official Journal of the European Union, Ci28/28, 6 June 2009.

Peter Hustinx. 'Opinion of the European Data Protection Supervisor on the current negotiations by the European Union of an Anti-Counterfeiting Trade Agreement (ACTA)'. Official Journal of the European Union, Ci47/I, 5 June 2010.

Annelies Huygen, Natali Heiberger, Joost Poort, Paul Rutten, and Nico Van Eijk. Ups and downs; economic and cultural effects of file sharing on music, film and games. http://www.tno.nl/ content.cfm?context=markten\&content=publicatie\&laagI=I82\&laag2=I\&item_id= $473 \&$ Taal $=2$, November is 2009. TNO Information and Communication Technology Series.

Philip Jones. 'Self-publishing helps pod take the lead in us'. The Bookseller.com, http://www. thebookseller.com/news/8589I-self-publishing-takes-the-lead-in-us.html, May 2009.

Michael Kanellos. 'Gates taking a seat in your den'. C-Net News, http://news.cnet.com/ Gates-taking-a-seat-in-your-den/2008-I04I_3-55I4I2I.html?tag|=mncol|;txt, 5 January 2005.

David Kravets. Copyright lawsuits plummet in aftermath of Riaa campaign. http://www.wired. com/threatlevel/2010/05/riaa-bump/, I8 May 2010.

La Quadrature du Net. Privacy: Film industry pirates European law. http://www.laquadrature. net/en/privacy-film-industry-pirates-european-law, I4 May 2008. 
La Quadrature du Net. EU governments united against the knowledge society? http://www. laquadrature.net/en/eu-governments-united-against-the-knowledge-society, I March 2011.

Frank La Rue. Report of the special rapporteur on the promotion and protection of the right to freedom of opinion and expression. United Nations Human Rights Council, http://www2.ohchr.org/ english/bodies/hrcouncil/docs/I7session/A.HRC.17.27_en.pdf, I6 May 2011.

Chantal Lacroix. 'Les dépenses de consommation des ménages en biens et services culturels et téléommunications'. DEPS, Ministère de la Culture, Pratiques et publics, CE2009-2, http://www2.culture.gouv.fr/culture/deps/2008/pdf/Cchiffresog_2.pdf,2009.

Stavros Lambrinidis. 'European parliament recommendation of 26 march 2009 to the council on strengthening security and fundamental freedoms on the internet'. Report adopted by the European Parliament, http://www.europarl.europa.eu/sides/getDoc.do?type= TA\&reference=P6-TA-2009-org4\&language=EN\&ring=A6-2009-0I03, 26 March 2009 .

U. Lee, M. Choi, J. Cho, M. Y. Sanadidi., and M. Gerla. Understanding pollution dynamics in p2p file sharing. In Proceedings of the $5^{\text {th }}$ International Workshop on Peer-to-Peer Systems (IPTPS o6), February 2006.

Gildas Lefeuvre. 'Les droits d'auteur restent stables mais fragilisés'. GL Connection newsletter, 77, January 2010.

Bruce A. Lehman. 'Intellectual property and the national information infrastructure: The report of the working group on intellectual property rights'. USPTO, http://www.uspto. gov/web/offices/com/doc/ipnii/ipnii.pdf, September 1995.

Lawrence Lessig. CODE and Other Laws of Cyberspace. Perseus Books Groups, I999. ISBN ASIN 046503912X.

Lawrence Lessig. The Future of Ideas: The Fate of the Commons in a Connected World. Random House, 200I. ISBN ISBN: 0375505784.

Lawrence Lessig. Free Culture: How Big Media Uses Technology and the Law to Lock Down Culture and Control Creativity. The Penguin Press, 2004. ISBN: 1594200068.

Lawrence Lessig. The Read-Write society. In Keynote speech at the Wizards of OS 4 Conference, 2006. http://www.wizards-of-os.org/index.php?id|=2322.

Stan J. Liebowitz. Testing file-sharing's impact by examining record sales in cities. http://somweb. utdallas.edu/centers/capri/documents/Impact_file_sharing.pdf, 2005. CAPRI Publication $05-02$.

James Love. Artists want to be paid: The Blur/Banff proposal. http://www.nsu.newschool.edu/ blur/bluro2/user_love.html,2002.

Mary Madden. Artists, musicians and the internet. http://www.pewinternet.org/Reports/2004/ Artists-Musicians-and-the-Internet.aspx, 2004.

Bruno Maresca, Romain Picard, Thomas Pilorin. 'Dépenses culture-médias des ménages au milieu des années 2000: une transformation structurelle'. DEPS/Ministère de la Culture. CE-20II-3. http://www2.culture.gouv.fr/culture/deps/2008/CE-20II-3-site.pdf

Emmi Martikainen. Does file-sharing reduce DVD sales? http://papers.ssrn.com/sol3/papers. cfm?abstract_id=I742443, September 20Io. also online at: http://taloustiede.utu.fi/hen kilokunta/henkilokunta/martikainen/MartikainenDVDpaper2oı.pdf.

Anna Masera. 'Accesso a internet, il paradosso italiano'. La Stampa Web, "http://www.lastam pa.it/_web/CMSTP/tmplrubriche/giornalisti/grubrica.asp?ID_blog=2\&ID_articolo= I045", 30 June 2010. 
François Moreau, Marc Bourreau, and Michel Gensollen. 'Quel avenir pour la distribution numérique des oeuvres culturelles'. Internet Actu, http://www.internetactu.net/2006/03/ 29/quel-avenir-pour-la-distribution-numerique-des-oeuvres-culturelles/,

29 March 2006.

Julie Holland Mortimer, Chris Nosko, and Alan Sorensen. 'Supply responses to digital distribution: Recorded music and live performances'. NBER Working Paper No. 16507, http:// mortimer.fas.harvard.edu/concerts_oroct2oro.pdf, October 2010.

Yann Moulier-Boutang. 'L'abeille et l'économiste'. Carnets Nord, May 2010. ISBN 9782355360305 .

Francis Muguet. Le mécénat global. http://mecenat-global.org/index-fr.html, septembre 2008. version 0.3 .

Neil Weinstock Netanel. 'Impose a Non-Commercial Use Levy to Allow Free P2P File-Sharing'. Harvard Journal of Law and Technology, I7(I), 2003.

Felix Oberholzer-Gee and Koleman Strumpf. 'The effect of file sharing on record sales: An empirical analysis'. Journal of Political Economy, II5(I), 2007.

Felix Oberholzer-Gee and Koleman Strumpf. 'File-Sharing and Copyright', pages I955. NBER Series, February 2010. online at http://musicbusinessresearch.files.wordpress. com/2010/06/paper-felix-oberholzer-gee.pdf.

Andrew Odlyzko. 'Providing security with insecure systems. In WiSec'ro: Proceedings of the Third ACM Conference on Wireless Network Security', pages 8788. ACM, 2010. Extended abstract.

Paul O'Mahony. 'Music industry: Make file sharing legal'. The Local: Swedish News in English, http://www.thelocal.se/9925/20080208/, February 2008.

Tim O'Reilly. 'Piracy is progressive taxation, and other thoughts on the evolution of online distribution'. O'Reilly openp2P.com, http://openp2p.com/pub/a/p2p/2002/I2/Ir/piracy. html, November 2002.

Andrew Orlowski. 'US students, alumni to get legal P2P: The beginning of the end of the file-sharing wars?' The Register, http://www.theregister.co.uk/2008/03/28/griffin_wmg _p2p_deall, 28 March 2008.

Will Page and Eric Garland. The Long Tail of P2P. http://www.prsformusic.com/creators/ news/research/Documents/The\% 2olong\%20tail\%200f\%20P2P\%20vg.pdf, May 2009.

Norman Paskin. 'The Digital Object Identifier (DOI) system'. Encyclopedia of Library and Information Sciences. Taylor \& Francis Group, 2008.

René Passet. 'L'économique et le vivant'. Economica, I996. ISBN 2-7178 3104-5. First edition in 1979.

M. Petruszewycz. 'L'histoire de la loi d'Estoup-Zipf: documents'. Mathématiques et sciences humaines, 44:4156, 1973. Accessible at http://www.numdam.org/numdambin/fitem?id= msh_I973_44_4I_o.

Heather Phillips. 'The great library of Alexandria?' Library Philosophy and Practice, Annual Volume, 20I0. http://unllib.unl.edu/LPP/phillips.htm.

Pour le cinéma. 'Lettre ouverte aux spectateurs citoyens'. Opinion editorial in Libération, initially signed by 13 key figures of the motion picture scene and later by 16 others, http://pourlecinema.over-blog.fr/pages/Lettre_ouverte_aux_spectateurs_citoyens-

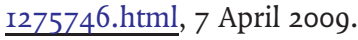


Redwood Capital. 'Sector overview report; digital music'. Redwood Capital, http://files.e2ma. net/13054/assets/docs/redwood_digital_music_January_2010.pdf, January 2010.

RIAA. Recording Industry Association of America Suggestions for content of ACTA. Published on the site of Knowledge Ecology International, http://www.keionline.org/index.php?option=com_con tent\&task=view\&id=Igo, June 2008.

Marco Ricolfi. 'Presentazione di background: Copyright 2.o'. In Conferenza NEXA su Internet \& Società, Torino, Italy, 28 November 2009. NEXA Center for Internet \& Society. http:// nexa.polito.it/conf2009.

Jeremy Rifkin. The Age of Access: The New Culture of Hypercapitalism, Where all of Life is a Paid-For Experience. Tarcher, 200I. ISBN 978 I585420827.

Christian Robin. Economies du droit d'auteur - I. Le livre. http://www2.culture.gouv.fr/culture/ deps/2008/pdf/Cetudeso7_4.pdf, December 2007.

SACEM. Rapport d'activité 2009 de la Société des auteurs, compositeurs et éditeurs de musique. http:// www.sacem.fr/files/content/sites/fr/files/mediatheque/sacem/presse/rapport_activite/ rapport_activite_2006.pdf, 2010.

Fabien Salliou. 'De la lutte contre le piratage au business du porno'. Libération Ecrans, septembre 2008. http://www.ecrans.fr/Media-defender,5229.html.

Richard E. Saunier and Richard A. Meganck, editors. Conservation of Biodiversity and the New Regional Planning. Organization of American States and The World Conservation Union, I995. ISBN o-8270-3592-6.

Clay Shirky. The case against micropayments. http://www.openp2p.com/pub/a/p2p/200o/I2/Ig/ micropayments.html, décembre 2000.

Clay Shirky. Power laws, weblogs, and inequality, 2003. http://www.shirky.com/writings/power law_weblog.html.

Felix Stalder. Variants of openness. Unpublished communication at the Openness: Code, Science, and Content Conference, http://I3I.193.153.231/www/issues/issueII_7/index. html, 2006.

Richard M. Stallman. 'The right way to tax DAT'. Wired magazine, May 1992.

Richard M. Stallman. Freedom or copyright. http://www.gnu.org/philosophy/freedom-or-copy right.html, 2009.

Joseph Stiglitz. 'Il faut un système pour inciter à la création du savoir'. Libération, http:// www.liberation.fr/culture/oror590982-il-faut-un-systeme-pour-inciter-a-la-creation-dusavoir, I5 September 2009.

John Sundman. 'The Pains'. Rosalita Associates, November 2008. ISBN 978-1929752003.

Tom F. Tan and Serguei Netessine. Is Tom Cruise threatened? using Netflix Prize data to examine the Long Tail of electronic commerce. http://knowledge.wharton.upenn.edu/papers/136r.pdf, 2009.

Tera-Equancy. Impact économique de la copie illégale des biens numérisés en France. Study conducted by Tera Consultants and Equancy \& Co, November 2008.

Alan Toner. Blur Banff proposal. P2P Foundation, http://p2pfoundation.net/Blur/Banff_Propo sal, January 2008.

David S. Touretzky. Gallery of CSS descramblers. http://www.cs.cmu.edu/ dst/DeCSS/Gallery/, 2004. Gallery curated by David Touretzky, individual works from dozens of contributors. 
UNU-MERIT. Study on the economic impact of open source software on innovation and the competitiveness of the information and communication technologies (ICT) sector in the EU.

http://ec.europa.eu/enterprise/sectors/ict/files/2006-II-20-flossimpact_en.pdf, November 2006. See pp. 123-I26 for the macro-economic figures.

Eliot Van Buskirk. 'Google wants spotify again'. Business Insider, http://www.businessinsi der.com/report-google-wants-to-acquire-spotify-again-20II-4, 22 April 2011.

B. Veenhof. The internet: Is it changing the way Canadians spend their time? Statistics Canada publication based on the 2005 time use study, http://www.statcan.gc.ca/pub/ 56 fooo4m/56fooo4m2006013-eng.pdf, August 2006.

H.L. Vogel. Entertainment Industry Economics: A Guide for Financial Analysis. Cambridge University Press, 2001.

Fred von Lohmann. A Better Way Forward: Voluntary Collective Licensing of Music File Sharing. http://www.eff.org/wp/better-way-forward-voluntary-collective-licensing-music-filesharing, 2003. The present on-line version has been updated since 2003.

Fred von Lohmann. 'Voluntary collective licensing for music file sharing'. Communications of the ACM, 47:2124, October 2004. http://portal.acm.org/ft_gateway.cfm?id= I022613\&type $=$ pdf.

George K. Zipf. The Psychobiology of Language. Houghton-Mifflin, I935.

Jonathan Zittrain. The Google/Verizon framework. The Future of the Internet and How to Stop It, http://futureoftheinternet.org/the-googleverizon-framework, I6 August 20I0. 



\section{Index}

A

absolute difference 188

absolute ranks 192

academia I02

academic research 177

access I46-I47, I53

access control I2I

access to education $\mathrm{I} 62$

access to knowledge $\quad$ I69

access without sharing $6 \mathrm{I}$

ACTA $24,50,74$

actors $\mathrm{I}_{62}$

ADAMI $68, \mathrm{I} 67, \mathrm{I} 75$

Adamic, Lada M. I8I

additional policy measures I4I, I43

AdSense 87, I43

advertising $45-46,55,60-6 \mathrm{I}$, I07, I42I43, I65

advocacy 173

Aidouni, Frédéric 189

Aigrain, Suzanne 190

Albanel, Christine 49

album 32, 37, 91-92, I06, I65, I7I, I75

Alliance Public Artistes 23

Alliance Public-Artistes $\quad 175$

alternative financing systems $17 \mathrm{I}$

alternative reward systems $67,69,75$

Amazon 6I

analog distribution and performance 158

Anderson, Chris 37, I8I, I9I-I92

Android 6I

Anitelli, Fernando 7, I65

Apple 6I, I63

archives 88, I8I

archiving 108

collaborative ro8

art market 108 artificial scarcity $\quad$ I42

artist income III

artists and repertoire 102

attention $\mathrm{I5}$

concentration 6o, 94, 96, I84

diversity $8,36,43-44,46,94, \mathrm{I} 8 \mathrm{I}$, I84, I89, I9I-I94, 203

economy 59

attribution 86, I2I

audience measurement intermediaries

I47

authors 68, II5-II6, I20, I3I

automatic recognition systems $\quad$ I49

average individual use 201

average readership per blog 203-204

B

Badin, Raphaël 7, I90

Balázs, Bodó 7, 43

Barcelona 77, I7I

Bautista, Eduardo 217

Bayart, Benjamin $\quad$ I63

Belgium 76, I29

Believe 165

Benkler, Yochai $7,45,74$

Berkman Center for Internet \&

Society@Berkman Center for

Internet \& Society 7

Bern Convention 86

best-selling artists $\quad \mathrm{I} 67$

bestselling authors 198

bibliometrics 184

BigChampagne 39

biodiversity $\mathrm{I} 32$

BitTorrent 32, 35, 40-44, 88, II $3, \mathrm{I}_{6}$

black hole scenario $44,49,107, \mathrm{I} 60$

blanket licensing, see\{license 8I

blog reading time 203 
blogs 29, 35, 69, 80, 82, 92, 98, IOI, I43, I46-I47, I53, I55, I72, I86, 203

Blu 90-9I

Blu-Ray II3

Blur/Banff proposal 79

Bogotaj, Maja 7,69

book photocopying 2I, 66, II8

book publishing I05-I06, III, II5, II8

books 27, 29, 35, 8I-82, 84, 88, IOI, II6, I22, I7I, I8I, I86

box office I03, II3, II5

Boyle, James 74, I7I

Branco, Juan 7

Branco, Paul 7

Brazil 76-77, I2I, I29, I65, I72, I76

broadcasters $\mathrm{I} 49, \mathrm{I} 72$

broadcasting 107

broadcasting by the public $\mathrm{I} 74$

budget $\mathrm{I} 66$

\section{C}

cable networks I42 $^{2}$

cam-cording 84

Canada I29, I75

capabilities 71, 74, 76, 98, I68

capability building 50,69, 174

Carasso, Jean-Gabriel 7

Carvalho, Aline 7

CDU 173

censorship I3I

centralized downloads $\mathrm{I}_{6}$

certification I $32^{2}$

Choruss 75

cinema II5

civic role $\mathrm{I} 77$

civil society $\mathrm{I} 73$

clarification 157

clues I47, I52-I55, I65, 200-202, 204205

co-operative financing systems 170

collaborative media 29

collaborative news media 69 , I02

collaborative rating 108

collecting $\mathbf{1 6 6}$ collecting societies $35,39,44,46,5 \mathrm{I}$, $66,68,76,83,97$, I39, I45, I5I, I64, I66-I67, I75-I76, 208

collective management 24,137, I66I67

collective means $\quad$ I68

collector sets 46

commenting I3I

Commission d'avance sur recettes II4 common good 82

common goods I6, 7I

commonism $\mathrm{I} 74$

commons I68

commons-based peer production $\quad$ I02

COMMUNIA Network 7

communism $\mathrm{I} 74$

compensation schemes, see

\{compensatory approaches 65

compensatory approaches $54,59,65$,

70, 89, I20, I28, I57

competition 69

competition policy I24, I4I, I58, I6I

competitive intermediaries 79, I04, I07, I60, I7I

composers 68

compulsory contribution 53

concentration of sales 46 , II 6

concert 46, 84, IIO, II2, I6I, I64-I65

concert tours $6 \mathrm{I}, 69$, II2

consultation processes 173

consumer groups 169

Consumer Protect on Technology I69

consumers 175

consumption

passive 75

personal expenditures for recreation

67

content sites I47, I53-I55

content-hosting Io8

continuum of positions $\quad$ I75

contract law $\mathrm{I}_{4} 2$

contracts $88, \mathrm{II} 8, \mathrm{I} 20, \mathrm{I} 59$

contributors $\mathrm{I} 2 \mathrm{O}, \mathrm{I} 65$

control $\mathrm{I} 62, \mathrm{I} 76$ 
convergence 8I

copyright law I6, 56, 69, 7I-72, 74, 77,

$83-84,92$, I09, I22

copyright reform 75, I2I, I7I-I72

copyright rewards 194

copyrightable databases $\quad$ 157-158

cost of operation 139

counter-arguments $157, \mathrm{I} 68$

Création-Public-Internet 77,173

Creative Commons 25, 29, 34-35, 69, $83,85-86,92$, I07, I2I, I59, I64-165, I70-17I, I89

creative communities $\quad$ I69

Creative Contribution I6, 53-55, 82-84, 86, 88-90, 94-95, 97, 99-102, 104, IO9, III-II2, II6-I2O, I22-I25, I27-

I29, I3I-I33, I37, I39, I42, I5I-I52,

I57-I58, I60-I6I, I64-I66, I7I, I73-

I74, I92-I94

creative ecosystem 49, 53, 167

creative writing workshops $9 \mathrm{I}$

credits 92-93

critical space 53

cross-checking 148

cryptographic integrity checks $15 \mathrm{I}$

cryptography 204

cultural commons 50, 54, 70-7I, 76,

82, I42, I67-I68, I70-I7I, I74

cultural craftsmanship I7I

cultural diversity $15,32-33,36-37,55$, 192

cultural ecosystem $\quad$ I69

cultural empowerment 32

cultural heritage 174

cultural industries $\mathrm{I} 6$

cultural industry era 75

cultural studies $\quad$ I84

cumulative distributions I88, I90

D

DADVSI $8 \mathrm{I}, 83$

dance $9 \mathrm{I}$

Darnton, Robert 88

data I02, 157 data collection agency $\quad$ 153, 204

data protection 24

databases 158

DC++ 43

de Martin, Juan Carlos 7

de-zoning 130

decentralized $\mathrm{P}_{2} \mathrm{P}$ model $\mathrm{I}_{3}$

decision-making processes I39, I4I, I67

DeCSS I2I

Deezer 46

Dehorter, Nicolas 104

democracy 173

democratic governance I39, I4I

derivative works 85

di Cosmo, Roberto 7, 92

differential contribution I30

differential pricing 130

digital distribution to the public 84

digital divulgation right $\quad 158$

digital photography 107

digital recordings III

digital sales $\quad$ I65, 202

Directive on Satellite Broadcasting and

Cable 85

distribution I6I-I62, I7I, I98, 200

distribution channels 175

distribution keys 80

distributive justice 69, III, I29, I74, 176

distributor monopoly 62

distributors 29, 45, 63, 65-66, 83, 85

diverse 154

DMCA I2I

Doctorow, Cory II6

documentary IOI, I07, II4

DOI I5I

donations 55, 165

Donnat, Olivier 98

Doueihi, Milad 7

download 29, 32, 38-40, 43, 45, 50, $59,62,64,87, \mathrm{II}_{3}, \mathrm{I} 47, \mathrm{I} 6_{3}$

downloads 4I, 45, II2

DRM 6o, 83, II5, I2I, I4I, I69, I75 
DRM-free II6

droit de retrait $\mathrm{I} 22$

dual digital society 76,162

due process $\mathrm{I}_{6} 2$

Dulong-de-Rosnay, Mélanie 7

DVD II8, I2I, I30

DVD sales II3-II4

Dylan, Bob 9I

\section{E}

eBook II5

economic rights 109

economies of scale 198

editorial activities I08

editorial selection I3I

eDonkey 35, 40, I89-I9o

Eldorado 43, 49, I62

electronic books 8I

Ellis, David 90-9I

emerging artists 68

empowerment I73-I74

eMule 4I, I9O

Endemol 33

entertainment industry $174-175$

equitable distribution $\quad$ I76

Estoup, Jean-Baptiste $\mathrm{I} 83-\mathrm{I} 84$

Europe $23-24,27,35,45,64-65,68$ -

$69,72-75,83,87,9$ I, 98, IOI, II3,

II9-I2I, I23, I42, I74, I76, 208, 2 II

European Copyright Directive of $200 \mathrm{I}$

$$
74,76
$$

European Directive on the Legal

Protection of Databases 158

exceptions 65,86

exceptions and limitations 34, 72-73,

IO9

exemplar 87-88, I22, I50

exhaustion of rights 72

see\{first sale doctrine 27

existence of works ro6

export sales I30 facts 157

fair dealing 33,72

fair trade I4I

fair trial $\quad \mathrm{I} 62$

fair use 33, 72, 86, I09

fake clues I52, I54-I55, 204-205

fake members I52, 204

fakes 3I, 40, I87, I90

Fanning, Shawn 30

farmers $\mathrm{I}_{32}$

FCC I42

feature film IOI

fees $55, \mathrm{I} 68$

file sharing, see\{sharing I5

film 8I, I05, II8, I65

film and audiovisual 50

film and video production $\quad$ IO4

film-makers $\quad$ I62

filtering $8 \mathrm{I}$

filters 102

financing of projects 90

financing schemes 23, 25, 177

financing sources 50

First Monday 169

first sale doctrine 27,72

Fisher, William T. 7, 23, 66, 70, 8I, 85-

86,89, I45-I 46,2 II

flat-rate $64-65,71,79-8$ I, 85, II6, II9,

I22, I45, I57, I65, I69-I72, I75-I76

Flattr I27, I53, I65-I66, I70

Flickr I07-108

for the benefit of a few $\quad$ I66

formality I5I

Forum d'Action Modernités 7

France $23-24,37,49,53,61,63-64$, 66, 68-69, 76-77, 8I, 83-85, 98-гоo, III, II3-II6, II8, I23-I24, I49, I53,

I55, I62, I70, I73, I75, 203

Franz, Vera 7

fraud I7, 86, 97, I39, I45-I49, I5I,

I53-I55, I6o, 204-205

in social systems $\quad$ I49

investigation 204 
presumption of $\mathrm{I} 49$

prevention and detection 204-205

Free Culture Forum 7, 77, I7I

Free Culture Research Conferences 177

Free Culture Research Workshop 2009

I9I

free/open source software I02, I52

freedom of expression $82, \mathrm{I} 62, \mathrm{I} 64$

freedom of information 172

freely reusable 157

Fuster-Morell, Mayo 7

\section{G}

gag law 172

games 8I, I0I, I05-106

Garland, Eric 39

gatekeepers 6o, I42

Gates, Bill I74, 2I9

GDP 45

geo-localization $\mathrm{I07}$

Germany 63, 76-77, 99-Ioo, I23-I24, I29, I64, I66

Ghosh, Rishab A. 96

global cultural welfare IIO-III, II9

GNU Free Documentation License 34

GNU General Public License version 3 205

Goldhaber, Michael 209

Google 6I-62, I42

GoogleBook settlement 64

governance I28, I39, I60, I66-I67, I7I,

I76

government-administered funds 103

government-administered rewards 66

Grassmuck, Volker 7

grassroots initiatives $\mathbf{I} 74$

grassroots intermediaries $\mathrm{I}_{5}$

Green 173

Grégoire 104

Griffin, Jim 7, 75, I75

guarantee fund 159

Guthrie, Woody 9I

\section{$\mathrm{H}$}

hacker spaces $9 \mathrm{I}$

HADOPI I50, I62-I63, I73

Hammond, John 9I

harmonic number $\mathrm{I} 86$

Harvard University I83, I9I

health 132

heirs of deceased artists 68,74

heirs of right holders $\quad$ I67

high-level benefits 149

home copying 72, I09, II2, II9

household

cultural expenditure I22-I24

expenditure for mobile communications I22, I24

fixed Internet access expenditure I22, I24

poorer households I24-I25

How-To for Sustainable Creativity I7I

human confirmation $\mathrm{I} 48$

Hyeronimi, Ruth 2I9

\section{I}

identifying works 149

IMPALA 64

in-situ biodiversity preservation $\quad$ I32

inclusion

of media 80,82

of works $82-83$

income $\mathrm{I} 82$

income progressiveness I25

income tax $\mathrm{I} 25$

independent artists, writers and

performers 107

independent impact studies 173

independent production $\mathrm{IO}_{3}, \mathrm{II} 4-\mathrm{II} 5$, I75

India II5

indications of interest 147

individual preferences 79, I59, I74

information services 107

innovative market offers $\quad \mathrm{I}_{4}$

integrity $\mathrm{I} 22$

intellectual rights 
interest groups 174

intermediaries 84,87

liability $24, \mathrm{I} 72$

intermediate absolute popularity rank

I92

intermediate popularity titles I4I

intermediate popularity works $\quad$ I92

international aspects I00, I29-I30

International Chamber of Commerce I69

Internet Archive OpenLibrary 36

Internet policy 169

Internet Service Providers 64, I22, I25, I38, I63, 208

Internet studies I84

Internet subscribing household 89

Internet-based participatory democracy

I 40

Internet-native media $43,67,69,80$

investigative reporting IOI, 107

investment funds 103

investors IIO, $\mathrm{I}_{7} 7$

iPad 62

iPhone 62

iPod IIO

ISBN I5I

Italy $53,76, \mathrm{I} 29$

iTunes 45, Io6, I63

J

Jamendo 92, 108

Japan II9

Jenner, Peter 7

Johansen, Jon Lech 2 I0

judicial procedure 82

K

keys 152

Kickstarter I03-I04, I56, I70

Knowledge Ecology International I69

knowledge, environment and social

commons $\quad$ I27
Kolmogoroff-Smirnov distance I88, I90

Kolmogoroff-Smirnov distance I9o

Krikorian, Gaëlle 7

Kulturflatrate I66, I73-I74

$\mathbf{L}$

La boîte verte $9 \mathrm{I}$

La Quadrature du Net 7, 207

labels 64, I03-104, I07, I65

laid-back entertainment $\quad$ I75

Lakatos, Zoltán 43

Lambrinidis, Stavros 2I8

Latapy, Mathieu 7, I89

Le Crosnier, Hervé 7, 208

Le Glatin, Marc 207

least-effort route $\quad \mathrm{I} 85$

Lessig, Lawrence 7, 74, I7I, I75

Levi, Simona 7

levies 55, II4, II8

on blank carriers 22, 72, II9-I2O

liability compensation 66

liberals $\quad \mathrm{I} 74$

librarians I8I

libraries 88

Library of Alexandria 30

Licence Art Libre 34

licence globale 23

license

blanket $23,8 \mathrm{I}$

collective 64

legal 62

licensees 64

licensing

blanket $\mathrm{I73}, \mathrm{I} 75$

collective $23,87-88$, I59

copyright licensing $\quad 65-66$

for commercial use $\mathrm{I}_{5} 5$

legal 65

statutory $64,83,87$, I 47

limited popularity titles I9I

linguistics $\mathrm{I} 84$

LIP6 I89

live performance $59, \mathrm{I} 49, \mathrm{I} 65$ 
living artists 68

locking-in 62

Long Tail theory 37, I8I, I9I

Love, James P. 7, 79, I69

\section{M}

magazine I22

Magnatune $83,92,165$

magnetic cassette tapes 22

Magnien, Clémence I89

majors 83, I03, II4, I75

making fraud less attractive 205

management costs $155^{-156}$

mandatory inclusion 83

manifestations of interest $\quad$ I47

many-to-all

creative world I5

cultural society $50,53,93,96$, I04, I 28

market segmentation $\quad 62,64$

mass registration $\mathrm{I} 5 \mathrm{I}$

media IOI

media chronology $84, \mathrm{II} 4, \mathrm{I} 30, \mathrm{I} 58$

media criticism 86

media industry III

media production 103

media publishing 193

MediaDefender 3I

Megaupload I63

memory institutions 88

merchandising 60

mere conduit $\mathrm{I} 72$

misgovernance 167

Miyet, Bernard 217

mobile communications $\quad$ I42

moderately popular works I53

modification of works $\quad$ I59

Moglen, Eben 7

monetizing the non-market $\quad \mathrm{I}_{6} 7$

monopoly price $\mathrm{I}_{62}$

moral rights 85, I2I-I 22

Mortimer, Julie Holland II2

motion picture 6I, I05, III, II3, I75

movie theaters 69 moving image 107

Muguet, Francis 7, 79

multimedia I05

music 98, IOI, I05, I07, IIO-III, I47,

I53-I54, I6I, I65, I7I, I86

music singles I54, $20 \mathrm{I}$

musical recording 52, 60, 68, 92, Io6

musicians III-II2

Musique Libre 38

mutualism 53

mutualization 53

mutualized financing I66

MyMajorCompany Io4

N

Napster 23, 30, 87

Nashville Skyline 92

NBC Universal II5

negotiating power I98

Nesson, Charles 7

Netessine, Serguei I9I

Netherlands II9, I29

network neutrality I42, I63, I76

news I0I, I07-I08, I2I, I3I, I42, I57, I65

Newscorp II5

newspaper Io6

NEXA Center for Internet \&

Society@NEXA Center for Internet \& Society 7

Noank Media 153

non-market I5, 28, 45, 47, 50, I54, I74

non-market commons I30

non-market use practices 148

normal exploitation $73-74$, II7

notoriety $\mathrm{I}_{6} 4$

novels IOI

0

O Teatro Mágico I65 $_{5}$

Oberholzer-Gee, Felix IIo

obfuscation 205

observation of Internet traffic I47, I53 
observed usage 196

occurrence of words 183

Odlyzko, Andrew I48, I87

offer of titles 44

oligopoly 6o-61, I75

omni-directional income channels 165

on-line encyclopedias 35

open publishing I3I

open society 173

Open Society Institute I3I

open standards I4I

opposition procedures 150

orphan editorial activities 107, 109, 128

orphan works 29, 35-36, 88, 158-159, I89

out-of-publication works 35-36,88, 158-159

O'Reilly (publisher) II8

$\mathbf{P}$

$\mathrm{P}_{2} \mathrm{P}$ clients 152

$\mathrm{P} 2 \mathrm{P}$ warfare $\mathrm{I}_{7} 7$

Page, Will 39

paper books II6

Pareto principle $\mathrm{I} 82$

Pareto, Vilfredo I8I

Pareto's law $18 \mathrm{I}-\mathrm{I} 83$

Paris Accord 169

participant 154

participative financing 127,160

participative intermediary $\mathrm{I} 66$

participative production 160,165

participatory production I04, 127

participatory rewards $\quad \mathrm{I}_{5}$

pecia 88

peer-managed funds 103

peer-to-peer

network 22, 3I-32, 35-36, 46

$\mathrm{P}_{2} \mathrm{P}$ file sharing 23, 29, 40, 46, IIо,

$$
\text { I46, I87, I89 }
$$

peer-to-peer file, $\mathrm{P}_{2} \mathrm{P}$ file sharing $\mathrm{I}_{3}$

performers 68,120

lead performers 68

support performers 68 periodical Io6

personal publishing 98

Petitgirard, Laurent 64, 217

Peugeot, Valérie 7

Phil Axel 7

philosophers II6

philosophical cafes 9I

phonorecord publishing 105-106

photo-reporting 107-108

photographic gallery 92

photographs 80, 82, 98, I0I, I08

photography 69, 107, I47

physical carriers 27

Pirate Bay 87, I27, I72

plug-ins 152

poetry 98

Pollock, Rufus 7

Pollstar II2

poorer countries 130

popularity I8I, 200

commercial I29

of creators 193

of works I5, 36, I54, I84

pornography $3 \mathrm{I}, \mathrm{I} 64$

possession I46-I47, I62

power law I83, I85-I87

Pragmazic 92, 108

precision I48, I53-155, 199

preferences, for support to production

I39

print on demand $\mathrm{I} 7 \mathrm{I}$

privacy $82,87,152,162$

private consumption

of cultural goods $5 \mathrm{I}$

of cultural industry goods $5 \mathrm{I}$

of music II2

privileges II5

probability density function 183

probability distribution $\quad 182$

producers $83,120,162$

product placement 60,143

production I6, 65, 76, 79-80, 89, 9I, I03, I09, III, II3-II5, I33, I60, I62, I64-165, I67, I75, I98 


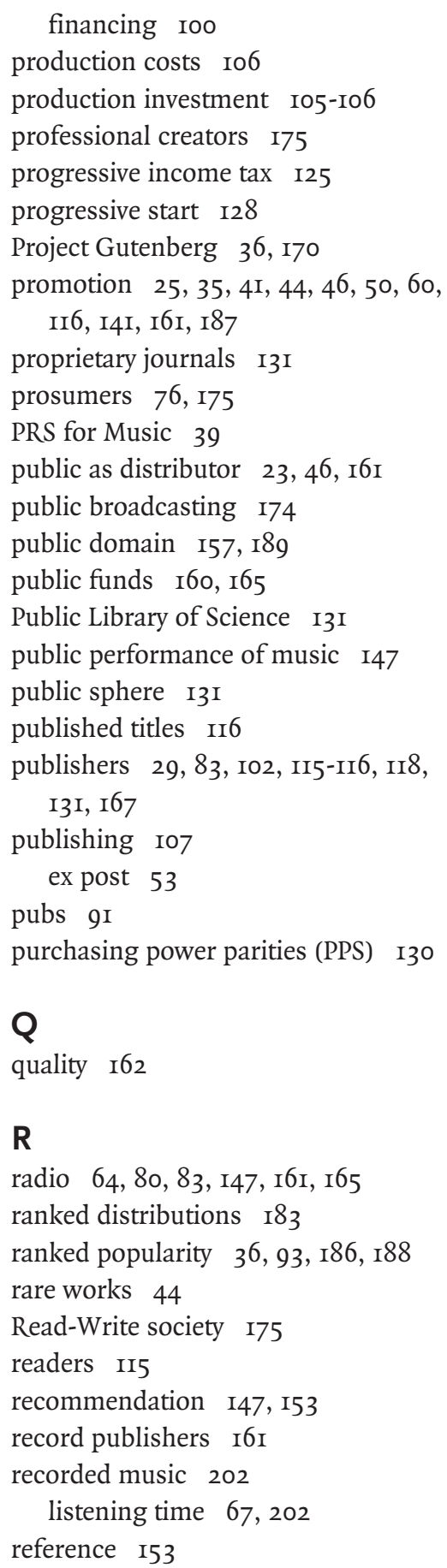

régime des intermittents du spectacle I 49

register 150

registered works 200-20I

registers $\mathrm{I} 38, \mathrm{I} 55$

registrant 150

registration I5O-I5I, I67

user 152

work I50, 203-204

registration of participants $\mathrm{I} 48$

relative use of works 153

remix rights 85,159

remixes 85

remuneration for work done 65

reported usage I93, I96

reporting I48, I52, I67, 204

repositories 88

repression I62, I72

resource pooling

organized 53

social 170

society-wide I3I, I7I, I74

statutory 53, I25, I29

voluntary 53

retail 69

revenues Io9, III-II3, II5-II6, II8

review I29

of use data 152

revision $\mathrm{I} 3 \mathrm{I}$

reward 65

calculation $\mathrm{I}_{67}$

financial rewards I6, I39, I42, I65

mechanism 50

minimum 93, 97

minimum relative level of use 199

model 54

system I6, 92

to the rewarders 148

total cost of rewards I93, I95-I97, 220

unit 92

usage-based rewards I6, 55, 80, 93

reward distribution system $\mathrm{I}_{6} 5$

reward function 7I, 92, 94, I97, 201 
cube root 197

less-than-proportional 94, 96-97,

I29, I66, I97-I98

power 2/3 I97

power function I98-I99

proportional 195

square root $\mathrm{I} 98$

reward system 93, I20, I59

constant cost 196, I99

reward@reward, model I5

rewards

ex-post 90

financial $\mathrm{I} 6$

usage-based 80

RIAA 67, I75, 208

Ricolfi, Marco 7

right holders, cartel 62

right to share $23,27,54,60-61,64-66$,

$69,74,76,79,82,85-86$, I09, I17-

II8, I20-I22, I37, I4I, I57-I58, I6I, I65-I66, I7I, I73

Rogard, Pascal 217

S

SACEM 44-46, 64, 68

salary $\mathrm{I} 68$

satellite I22

scale invariance $\quad$ I87

scanning 84

scarcity

of copies 49, 82, III, II3, I6I, I75

of titles 175

science $\mathrm{IO} 2$

scientific publications 35,82 , I02, I57-

I58, I70

scientific publishing 88, I3I, I58

scores $\quad \mathrm{I} 62$

search engines 107

seed banks I32

seeds $\mathrm{I}^{2} 2$

self-production I7I

Sellaband I04

sharing compatible 49, 53, III, I2I, I42, I69, I7I, I75

de facto $33-34$

decentralized $\mathrm{I}_{6}$

impact $43,49-50,67-68$, IIo

unauthorized 33, 35, I6I, I75, I89, I93

voluntary 29, 33-34, 67, I08, I6I, I69-I7I, I9I

Shazam I50

short videos 80

SIAE I75

skill 94

Slashdot 69

SNE II6

social acceptability $\quad$ I22, I25

social and health insurance I49

social pact 56,77

social public goods I32, I68

social rights $\mathrm{I} 6,54,59,70,72, \mathrm{I}_{3} 8$

social-democrats $\quad \mathrm{I} 74$

SOFICA II4

software I02, I57-I58

Sony II5

Spain 72

spam robots 148

SPD 173

specialized currency creation $\quad$ I32

SPEDIDAM I67, I75

Spidart I04

split between media I37, I39, I59

sponsorship 55

Spotify 46, 62-63

squats 9I

Stallman, Richard M. 7, 22, 97, I97

statutory payment I25, I66

Stiglitz, Joseph 92

STIM 76, I67

stock archives I07-I08

streaming $32-33,46,59-60,62,64,87$, II $3, I_{3}$

Strumpf, Koleman IIO

subscription 45-46, 55, 60, 62-63, I65

Sunde, Peter 7, I27, I70 
Sundman, John II6

supply pattern 50

surveillance $\mathrm{I} 62$

surveys I39, I48

sustainable financing $\quad$ I27

Sweden 76, 96, I29, I75

T

\section{TACD 77}

tagging I50

takings doctrine II7-II8

Tan, Tom F. IgI

$\operatorname{tax} \mathrm{I} 66$

tax credits II4

teaching 46

technical protection measures 60

television $5 \mathrm{I}$

texts IOI

theater plays IoI

theater projection 46 , II 4

theater tickets II4

three-step test $73,83, \mathrm{II} 7$

three-strike approaches 24, 8I, I62, I69

threshold of practical usefulness 98

time budget 7I, I48

Time Warner II5

titles I6I, 20I

top-down cultural policy $\mathbf{I 7 4}$

topping-off 96, III, I97

Touscoprod IO4

TPM I2I

Trama Virtual I65 $_{5}$

transaction costs $28,51,83,92,97$, I32, I5I, I65, I67

transition scheme $\mathrm{I} 20$

transmission, of use data $\mathrm{I}_{52}$

transparency I39, I6o

trust 205

TV 8I, I05, II3-II5, I22, I65

TV series 92

TV-based II6
U

UDHR 2I, 27

UK II6, I29

unfair trade I4I

Uniform Resource Identifier (URI) 150

unique identification code 150

universe size I95

University of Exeter 190

upload 43, I46-I47, I53

US $23,45,61,63,67-68,72,75-76,83$, 9I, 98-I00, Io6, II4-II6, I2I, I23-

I24, I29, I42, I50, I53-I54, I70-I7I,

I74-I75, 20I, 208

US Copyright Act of $1976 \quad 72$

US Copyright Register 150

usage

diversity I93, I96

measurement I5-I6, 97, I40, I45, I56, I58, I60, I67, I99, 205

usage data I39, I66, I76

usage measurement 199

USB keys 29-30, 32, 43, II3

user preferences $\mathrm{I} 67$

user prerogatives 72,86

user-generated content sites I53

\section{V}

vegetal varieties 132

Verizon I42

Vermont I37

vertical integration 62

Viacom II5

video 8I, IOI, IIO, II3, II8, I65, I75

video and TV 105

video-bloggers 172

virtual corporations 137

visual art IOI, I04-I05

voluntary participatory rewards $\mathrm{I}_{5}$

voluntary sharing communities $\mathrm{I} 53$

voluntary user sample I46, I48, I52-

I53, I55, I6o, I99-200, 202 


\section{W}

Walt Disney II5

war against piracy 65,173

war against sharing $24,43-44,64,75$, 8I, 168

Warner Music 75, I75

wealth $\mathrm{I} 8 \mathrm{I}-\mathrm{I} 82$, I85

wealth distribution $\mathrm{I} 8 \mathrm{I}-\mathrm{I} 82$

Web $\quad$ I52

Wesnigk, Cay 218

Wharton Business school I9I

Wikipedia 69, I8I

WikiSource 36

WIPO 34

WIPO 1996 treaties II9
WordPress I49, I55

writers III, II5

writing II4

Y

Yooook 170

Z

Zelnik, Patrick 64 Zimmermann, Jérémie 7 Zipf, George K. 37, I83, I85 Zipf's law 37-38, 40, 93-94, 96, I8I, I83-I97, I99-201, 203 